J. DIFFERENTIAL GEOMETRY

62 (2002) 351-559

\title{
ASYMPTOTIC BEHAVIOUR OF TAME NILPOTENT HARMONIC BUNDLES WITH TRIVIAL PARABOLIC STRUCTURE
}

\author{
TAKURO MOCHIZUKI
}

\begin{abstract}
Let $E$ be a holomorphic vector bundle. Let $\theta$ be a Higgs field, that is a holomorphic section of End $(E) \otimes \Omega_{X}^{1,0}$ satisfying $\theta^{2}=0$. Let $h$ be a pluriharmonic metric of the Higgs bundle $(E, \theta)$. The tuple $(E, \theta, h)$ is called a harmonic bundle.

Let $X$ be a complex manifold, and $D$ be a normal crossing divisor of $X$. In this paper, we study the harmonic bundle $(E, \theta, h)$ over $X-D$. We regard $D$ as the singularity of $(E, \theta, h)$, and we are particularly interested in the asymptotic behaviour of the harmonic bundle around $D$. We will see that it is similar to the asymptotic behaviour of complex variation of polarized Hodge structures, when the harmonic bundle is tame and nilpotent with the trivial parabolic structure. For example, we prove constantness of general monodromy weight filtrations, compatibility of the filtrations, norm estimates, and the purity theorem.

For that purpose, we will obtain a limiting mixed twistor structure from a tame nilpotent harmonic bundle with trivial parabolic structure, on a punctured disc. It is a solution of a conjecture of Simpson.
\end{abstract}

\section{Introduction}

\subsection{Harmonic bundles}

Let $X$ be a complex manifold. Let $\left(E, \bar{\partial}_{E}\right)$ be a holomorphic bundle. Let $\theta$ be a Higgs field of $E$, namely, it is a holomorphic section of End $(E) \otimes \Omega^{1,0}$ satisfying $\theta \wedge \theta=0$. Let $h$ be a hermitian metric of $E$. Let $\partial_{E}$ denote the $(1,0)$-part of the metric connection of $\left(E, \bar{\partial}_{E}, h\right)$.

Received 07/09/2002. 
We also have the adjoint $\theta^{\dagger}$ of $\theta$ with respect to the metric $h$. Then we obtain the following connection:

$$
\mathbb{D}^{1}:=\bar{\partial}_{E}+\partial_{E}+\theta+\theta^{\dagger}: C^{\infty}(X, E) \longrightarrow C^{\infty}\left(X, E \otimes \Omega_{X}^{1}\right) .
$$

More generally, we obtain the following $\lambda$-connection for any $\lambda \in \mathbf{C}$ :

$$
\mathbb{D}^{\lambda}:=\bar{\partial}_{E}+\theta+\lambda \cdot\left(\partial_{E}+\theta^{\dagger}\right): C^{\infty}(X, E) \longrightarrow C^{\infty}\left(X, E \otimes \Omega_{X}^{1}\right) .
$$

Definition 1.1. The metric $h$ is called pluriharmonic, if the connection $\mathbb{D}^{1}$ is flat, that is, $\mathbb{D}^{1} \circ \mathbb{D}^{1}=0$. The tuple $\left(E, \bar{\partial}_{E}, \theta, h\right)$ is called a harmonic bundle.

Note that the condition is equivalent to " $\mathbb{D}^{\lambda} \circ \mathbb{D}^{\lambda}=0$ for all of $\lambda "$. We have $\mathbb{D}^{0}=\bar{\partial}_{E}+\theta$, and the condition $\mathbb{D}^{0} \circ \mathbb{D}^{0}=0$ is equivalent to the condition that $\theta$ is a Higgs field.

Remark 1.1. Probably, such object should be called a 'pluriharmonic bundle'. However we use 'harmonic bundle' for simplicity.

Let $D$ be a normal crossing divisor of a complex manifold $X$. In this paper, our main interest is a harmonic bundle over $X-D$, and we investigate the asymptotic behaviour of the harmonic bundle around $D$. We will impose the following conditions (see Subsection 4.2 for more detail):

Condition 1.1. Let $P$ be any point of $X$, and $(\mathcal{U}, \varphi)$ be an admissible coordinate around $P$ (Definition 4.1). On $\mathcal{U}$, we have the description:

$$
\theta=\sum_{j=1}^{l} f_{j} \cdot \frac{d z_{j}}{z_{j}}+\sum_{j=l+1}^{n} g_{j} \cdot d z_{j} .
$$

Tameness. Let $t$ be a formal variable. We have the polynomials $\operatorname{det}\left(t-f_{j}\right)$ and $\operatorname{det}\left(t-g_{j}\right)$ of $t$, whose coefficients are holomorphic functions defined over $\mathcal{U}-\bigcup_{j=1}^{l} D_{i_{j}}$. When the functions are extended to the holomorphic functions over $\mathcal{U}$, the harmonic bundle is called tame at $P$.

Nilpotentness. Assume that the harmonic bundle is tame at $P$. When $\left.\operatorname{det}\left(t-f_{j}\right)\right|_{\mathcal{U} \cap D_{i_{j}}}=t^{r}$, then the harmonic bundle is called nilpotent at $P$.

When $\left(E, \bar{\partial}_{E}, h, \theta\right)$ is a tame nilpotent at any point $P \in X$, then it is called a tame nilpotent harmonic bundle. 
Trivial parabolic structure. We say that the parabolic structure of $\left(E, \bar{\partial}_{E}, \theta, h\right)$ is trivial, if the parabolic structure of the prolongment of the restriction $\left(E, \bar{\partial}_{E}, \theta, h\right)_{\mid C}$ is trivial for any holomorphic curve $C$ transversal with $D$. (See Condition 4.1 and Definition 4.5.)

In the words of the flat bundle $\left(E, \mathbb{D}^{1}\right)$, the combination of the nilpotentness condition and the triviality of the parabolic structures are described as follows:

\section{Condition 1.2.}

1. The monodromies around the components of $D$ are unipotent.

2. Let $s$ be a multi-valued flat section. Let $(\mathcal{U}, \varphi)$ be an admissible coordinate around $P$. Then we have equalities $0<C_{1} \cdot \prod_{i=1}^{l}\left|z_{i}\right|^{\epsilon} \leq$ $|s|_{h} \leq C_{2} \cdot \prod_{i=1}^{l}\left|z_{i}\right|^{-\epsilon}$ for any $\epsilon>0$. (Precisely, we need only the estimate on curves.)

Recall that harmonic bundle can be regarded as a generalization of complex variation of polarized Hodge structures (CVHS). On CVHS, the highly developed theories for the asymptotic behaviour are well-known due to Cattani-Kaplan-Schmid and Kashiwara-Kawai. Briefly and imprecisely speaking, their results say that we have some nice relations between the monodromies, and that the monodromy weight filtrations describe the asymptotic behaviour. Although their results indicate the direction of our study, it seems difficult to apply directly their method in our case, for their methods heavily use the Hodge filtrations. But some of techniques and lemmas are still efficient in our study.

When the base manifold $X$ is one dimensional, such behaviour was deeply studied by Simpson. Moreover, he proposed the 'mixed twistor structure', which is quite important for the study in the case $X$ is higher dimensional. In fact, most of the essential ideas contained in this paper are due to Simpson (see [34], [35], [36] and [37]): We will heavily owe to many results and methods that he developed in [34] and [35]. We will often use them without mention his name. The papers are fundamental for our study of harmonic bundles. The mixed twistor structure was introduced in [36]. (The original twistor setup for weight 1 was due to Hitchin and Deligne.) The mixed twistor structure permits us to obtain some compatibilities on the relations between the monodromy weight filtrations at the intersection points of divisors. (Such compatibilities are well-known for the complex variation of the polarized Hodge structure.) 
It seems difficult for the author to obtain such results, if we use only some rather classical elliptic analytic argument without mixed twistor structure.

\subsection{Main results}

Recall that the harmonic bundle can be regarded as a generalization of the complex variation of Hodge structure. Briefly speaking, our final but unreached purpose in this study is to see the following:

The asymptotic behaviour of a tame harmonic bundle around the singularity is similar to the behaviour of CVHS around the singularity, in some sense.

As is already noted, we will mainly investigate the tame harmonic bundles under the assumptions of nilpotentness and the triviality of parabolic structures. We explain our results when the dimension of the base manifold is two in this subsection.

\subsubsection{Flat connection}

Since we are interested in the asymptotic behaviour around the singularity of harmonic bundles, we can assume that $X=\Delta^{2}=\left\{\left(z_{1}, z_{2}\right)|| z_{i} \mid<\right.$ $1\}$ and $D=D_{1} \cup D_{2}$. Here we put $D_{i}:=\left\{z_{i}=0\right\}$. Let $\left(E, \bar{\partial}_{E}, \theta, h\right)$ be a tame nilpotent harmonic bundle with trivial parabolic structure over $X-D$. Let $P$ be a point of $X-D$. We have the loop $\gamma_{i}:[0,1] \longrightarrow X-D$ defined as follows:

$$
z_{j}\left(\gamma_{i}(t)\right)= \begin{cases}z_{i}(P) \cdot \exp (2 \pi \sqrt{-1} t) & (j=i) \\ z_{j}(P) & (j \neq i) .\end{cases}
$$

We put $V=E_{\mid P}$. We have the monodromy $M\left(\gamma_{i}\right) \in \operatorname{End}\left(E_{\mid P}\right)$ with respect to the flat connection $\mathbb{D}^{1}$ given in (1). Due to our assumption, it is unipotent. Thus we have the $\operatorname{logarithm} N_{i}=\log M\left(\gamma_{i}\right)$. We put $N(\boldsymbol{a})=\sum_{i=1}^{2} a_{i} \cdot N_{i}$ for $\boldsymbol{a}=\left(a_{1}, a_{2}\right) \in \mathbf{R}_{>0}^{2}$. Let $W(\boldsymbol{a})$ denote the weight filtration of $N(\boldsymbol{a})$. We have the constantness of the filtration on the positive cones. Namely, the following holds.

Theorem 1.1. We have $W\left(\boldsymbol{a}_{1}\right)=W\left(\boldsymbol{a}_{2}\right)$ for any $\boldsymbol{a}_{i} \in \mathbf{R}_{>0}^{2}$.

We put $N(\underline{1})=N_{1}$ and $N(\underline{2})=N_{1}+N_{2}$. We denote the weight filtration of $N(\underline{j})$ by $W(\underline{j})$. Let $\mathcal{G} r^{(1)}$ denote the associated graded 
space of $W(\underline{1})$. Then we have the induced filtration $W^{(1)}(\underline{2})$ on $\mathcal{G} r^{(1)}$. On the other hand, we have the induced action $N^{(1)}(\underline{2})$ on $\mathcal{G} r^{(1)}$. Let $W\left(N^{(1)}(\underline{2})\right)$ denote the weight filtration of $N^{(1)}(\underline{2})$. Namely, we have two filtrations $W^{(1)}(\underline{2})$ and $W\left(N^{(1)}\right)(\underline{2})$ on the graded vector space $\mathcal{G} r^{(1)}=$ $\bigoplus_{h} \mathcal{G} r_{h}^{(1)}$.

Theorem 1.2. We have $W^{(1)}(\underline{2})_{a+h} \cap \mathcal{G} r_{a}^{(1)}=W\left(N^{(1)}(\underline{2})\right)_{h} \cap \mathcal{G} r_{a}^{(1)}$.

Theorem 1.1 and Theorem 1.2 are generalization of the results due to Cattani-Kaplan in [5].

We put $\mathbb{H}:=\{\zeta=x+\sqrt{-1} y \in \mathbf{C} \mid y>0\}$. Then we have the universal covering $\pi: \mathbb{H}^{2} \longrightarrow X-D$ defined by

$$
\pi\left(\zeta_{1}, \zeta_{2}\right)=\left(\exp \left(2 \pi \sqrt{-1} \zeta_{1}\right), \exp \left(2 \pi \sqrt{-1} \zeta_{2}\right)\right) .
$$

We put as follows for positive numbers $A$ and $C$ :

$$
\begin{aligned}
\widetilde{Z}(\mathrm{id}, 2, C, A):=\left\{\left(x_{1}+\sqrt{-1} y_{1},\right.\right. & \left.x_{2}+\sqrt{-1} y_{2}\right) \in \mathbb{H}^{2} \mid \\
& \left.\left|x_{1}\right|<A,\left|x_{2}\right|<A, C \cdot y_{1}>y_{2},\right\} .
\end{aligned}
$$

Let $\widetilde{P}$ be a point of $\widetilde{Z}(\mathrm{id}, 2, C, A)$. We have the pull back $\pi^{*}\left(E, \mathbb{D}^{1}, h\right)$. The fibers $\pi^{*}(E)_{\mid \widetilde{P}}$ and $E_{\mid P}$ are naturally identified.

Let $u$ be a nonzero element of $E_{\mid P}$. Then we have the numbers $h_{j}=\operatorname{deg}^{W(\underline{j})}(u)$ for $j=1,2$. Let $f$ be a flat section of $\left(\pi^{*} E, \mathbb{D}^{1}\right)$ such that $f_{\mid \widetilde{P}}=u$.

Theorem 1.3. There exist positive numbers $C_{1}$ and $C_{2}$ such that the following inequality holds on $\widetilde{Z}(\mathrm{id}, 2, C, A)$ :

$$
0<C_{1} \leq|f|_{h}^{2} \cdot y_{1}^{h_{1}} \cdot y_{2}^{h_{2}-h_{1}} \leq C_{2} .
$$

Theorem 1.3 is generalization of the results in [6] and [25].

From the tuple $\left(V, N_{1}, N_{2}\right)$, we obtain the following complex:

$$
\Pi\left(N_{1}, N_{2}\right): V \stackrel{d}{\longrightarrow} \operatorname{Im}\left(N_{1}\right) \oplus \operatorname{Im}\left(N_{2}\right) \stackrel{d}{\longrightarrow} \operatorname{Im}\left(N_{1} N_{2}\right) .
$$

It is easy to see $H^{0}\left(\Pi\left(N_{1}, N_{2}\right)\right)=\operatorname{Ker}\left(N_{1}\right) \cap \operatorname{Ker}\left(N_{2}\right)$ and $H^{2}\left(\Pi\left(N_{1}, N_{2}\right)\right)$ $=0$. The filtration $W(\underline{2})$ on $V$ induces the filtration $W$ of the complex as follows:

$$
\begin{gathered}
W_{k}(V)=W(\underline{2})_{k}, \quad W_{k}\left(\operatorname{Im}\left(N_{i}\right)\right)=N_{i}\left(W(\underline{2})_{k}\right), \\
W_{k}\left(\operatorname{Im}\left(N_{1} N_{2}\right)\right)=N_{1} N_{2}\left(W(\underline{2})_{k}\right) .
\end{gathered}
$$

It induces the filtration $W$ on the cohomology group $H^{*}\left(\Pi\left(N_{1}, N_{2}\right)\right)$. 
Theorem 1.4 (Purity theorem). Assume that $\left(E, \bar{\partial}_{E}, \theta, h\right)$ has a real structure. We have $W_{k} H^{k}\left(\Pi\left(N_{1}, N_{2}\right)\right)=H^{k}\left(\Pi\left(N_{1}, N_{2}\right)\right)$. In other words, the naturally defined morphisms $\operatorname{Ker}(d) \cap W_{k}\left(\Pi\left(N_{1}, N_{2}\right)^{k}\right) \longrightarrow$ $H^{k}\left(\Pi\left(N_{1}, N_{2}\right)\right)$ are surjective.

Remark 1.2. Due to Cattani-Kaplan-Schmid and Kashiwara-Kawai, the intersection cohomology and the $L^{2}$-cohomology of CVHS are isomorphic ([7] and [25]). The purity theorem was crucially used for the proof of such coincidence. Although we do not discuss the relation between the intersection cohomology and the $L^{2}$-cohomology of harmonic bundles in this paper, it seems appropriate to include the purity theorem here. The author intends to study the relations between $L^{2}$-cohomology and the intersection cohomology in another paper.

\subsubsection{Holomorphic bundles}

In the previous subsubsection, we state the results in terms of the flat bundles and the monodromies. It is reworded in terms of the holomorphic flat bundles and the residues. The $(0,1)$-part $\bar{\partial}+\lambda \cdot \theta^{\dagger}$ of the connection $\mathbb{D}^{1}$ gives a holomorphic structure $d^{\prime \prime 1}$ to the $C^{\infty}$-vector bundle $E$. We denote the holomorphic bundle $\left(E, d^{\prime \prime}\right)$ by $\mathcal{E}^{1}$. Let ${ }^{\diamond} \mathcal{E}^{1}$ denote the prolongment of $\mathcal{E}^{1}$ by an increasing order. (See Subsection 4.1.) We can show that ${ }^{\diamond} \mathcal{E}^{1}$ is locally free and same as the canonical extension, i.e., $\mathbb{D}^{1}$ is of log type on ${ }^{\diamond} \mathcal{E}^{1}$.

We have the residues $N_{i}:=\operatorname{Res}_{D_{i}}\left(\mathbb{D}^{1}\right) \in \Gamma\left(D_{i}, \operatorname{End}\left({ }^{\diamond} \mathcal{E}^{1} \mid D_{i}\right)\right)$ for $i=1,2$. We put $D_{1}=D_{1}$ and $D_{\underline{2}}=D_{1} \cap D_{2}$. For $\boldsymbol{a} \in \mathbf{R}_{>0}^{2}$, we put $N(\boldsymbol{a}):=\sum_{i=1}^{2} a_{i} \cdot N_{i \mid D_{2}}$. Let $W(\boldsymbol{a})$ denote the weight filtration of $N(\boldsymbol{a})$. Theorem 1.1 is reworded as follows:

Theorem 1.5 (Theorem 7.1). We have $W\left(\boldsymbol{a}_{1}\right)=W\left(\boldsymbol{a}_{2}\right)$ for any $\boldsymbol{a}_{i} \in \mathbf{R}_{>0}^{2}$.

On $D_{1}$, we put $N(\underline{1})=N_{1}$. Let $W(\underline{1})$ denote the weight filtration of $N(\underline{1})$, which is a filtration of ${ }^{\diamond} \mathcal{E}^{1}{ }_{\mid D_{1}}$ by vector subbundles. We obtain the graded vector bundle $\mathcal{G} r^{(1)}$ on $D_{\underline{1}}$.

On $D_{2}$, we put $N(\underline{2})=\sum_{i=1}^{2} N_{i \mid D_{i}}$. Let $W(\underline{2})$ denote the weight filtration of $N(\underline{2})$, which is a filtration of ${ }^{\diamond} \mathcal{E}^{1} D_{\underline{2}}$.

Consider $\mathcal{G} r_{\mid D_{\underline{1}}}^{(1)}$. The filtration $W(\underline{2})$ of $\diamond \mathcal{E}^{1}{ }_{\mid D_{\underline{2}}}$ induces the induced filtration $W^{(1)}(\underline{2})$ of $\mathcal{G} r_{\mid D_{\underline{1}}}^{(1)}$. On the other hand, $N(\underline{2})$ induces the endomorphism $N^{(1)}(\underline{2})$ of $\mathcal{G} r_{\mid D_{2}}^{(1)}$. Then we obtain the weight filtration 
$W\left(N^{(1)}(\underline{2})\right)$. Theorem 1.2 is reworded as follows:

Theorem 1.6 (Theorem 8.1). We have

$$
W^{(1)}(\underline{2})_{h+a} \cap \mathcal{G} r_{a}^{(1)}=W\left(N^{(1)}(\underline{2})\right)_{h} \cap \mathcal{G} r_{a}^{(1)}
$$

for any integers $a$ and $h$.

We will see more strong compatibility in Theorem 8.2. Clearly, we can also replace the role of $N_{1}$ and $N_{2}$ (Theorem 8.3).

Take a holomorphic frame $\boldsymbol{v}=\left(v_{1}, \ldots, v_{r}\right)$ of ${ }^{\diamond} \mathcal{E}^{1}$ over $X$, compatible with the sequence of the filtrations $(W(\underline{1}), W(\underline{2}))$. Namely it satisfies the following:

- $\boldsymbol{v}_{\mid D_{\underline{j}}}$ is compatible with the filtration $W(\underline{j})$ over $D_{\underline{j}}$. In particular, we obtain the induced frame $\boldsymbol{v}^{(1)}$ over $D_{\underline{1}}$.

- $\boldsymbol{v}_{\mid D_{2}}^{(1)}$ is compatible with $W^{(1)}(\underline{2})$.

- We have $\operatorname{deg}{ }^{W(\underline{2})}\left(v_{i}\right)=\operatorname{deg} W^{(1)}(\underline{2})\left(v_{i}^{(1)}\right)$.

We put $2 \cdot k_{1}\left(v_{i}\right):=\operatorname{deg}^{W(\underline{1})}\left(v_{i}\right)$ and $2 \cdot k_{2}\left(v_{i}\right):=\operatorname{deg}^{W(\underline{2})}\left(v_{i}\right)-\operatorname{deg}^{W(\underline{1})}\left(v_{i}\right)$. We obtain the $C^{\infty}$-frame $\boldsymbol{v}^{\prime}=\left(v_{1}^{\prime}, \ldots v_{r}^{\prime}\right)$ of $\mathcal{E}^{1}$ over $X-D$ defined as follows:

$$
v_{i}^{\prime}:=v_{i} \cdot\left(-\log \left|z_{1}\right|\right)^{-k_{1}\left(v_{i}\right)} \cdot\left(-\log \left|z_{2}\right|\right)^{-k_{2}\left(v_{i}\right)} .
$$

For a positive number $C$, we put as follows:

$$
Z(\mathrm{id}, 2, C):=\left\{\left(z_{1}, z_{2}\right) \in X-\left.D|| z_{1}\right|^{C}<\left|z_{2}\right|\right\} .
$$

The following theorem is an analogue of Theorem 1.3.

Theorem 1.7 (Theorem 9.1). On $Z(\mathrm{id}, 2, C)$, the $C^{\infty}$-frame $\boldsymbol{v}^{\prime}$ is adapted. Namely the hermitian matrix-valued functions $H\left(h, \boldsymbol{v}^{\prime}\right)$ and the inverse $H\left(h, \boldsymbol{v}^{\prime}\right)^{-1}$ are bounded over $Z(\mathrm{id}, 2, C)$.

Clearly we can replace the roles of 1 and 2 (Theorem 9.2).

We put $\mathcal{V}={ }^{\diamond} \mathcal{E}^{1}{ }_{\mid D_{\underline{2}}}$ and $\mathcal{N}_{i}:=N_{i \mid D_{\underline{\underline{2}}}}$. Theorem 1.4 is reworded as follows:

Theorem 1.8 (Theorem 9.6). Assume that $\left(E, \bar{\partial}_{E}, \theta, h\right)$ has a real structure. The purity theorem for $\left(\mathcal{V}, \mathcal{N}_{1}, \mathcal{N}_{2}\right)$ holds. 
In the last of the paper (Subsection 9.2), we obtain "limiting CVHS" of a tame nilpotent harmonic bundle with trivial parabolic structure. The author expects that it is a useful tool when we would like to reduce the study of tame harmonic bundle to the study of CVHS. In fact, Theorem 1.8 is shown in such a way. (See Subsubsection 9.2.3.) A limiting CVHS is obtained as follows: Let $\boldsymbol{v}$ be a holomorphic frame of ${ }^{\diamond} \mathcal{E}^{1}$, which is compatible with $W(\underline{2})$ on $D_{\underline{2}}$. We put $2 \cdot k\left(v_{i}\right):=$ $\operatorname{deg}^{W(2)}\left(v_{i}\right)$. Consider the morphism $\psi_{m, \underline{2}}: X-D \longrightarrow X-D$ defined by $\psi_{m, \underline{2}}\left(z_{1}, z_{2}\right)=\left(z_{1}^{m}, z_{2}^{m}\right)$. We have the pull back $\psi_{m, 2}^{*}\left(\mathcal{E}^{1}, \mathbb{D}^{1}, h\right)$. We have the frame $\boldsymbol{v}^{(m)}$ of $\psi_{m, \underline{2}}^{*} \mathcal{E}^{1}$ defined as follows:

$$
v_{i}^{(m)}=\psi_{m, \underline{2}}^{*}\left(v_{i}\right) \cdot m^{-k\left(v_{i}\right)} .
$$

Let $F=\bigoplus_{i=1}^{r} \mathcal{O}_{\Delta^{*}} \cdot u_{i}$ denote the trivial holomorphic bundle with the frame $\boldsymbol{u}=\left(u_{i}\right)$. We denote the holomorphic structure of $F$ by $d_{F}^{\prime \prime}$. Due to the frames $\boldsymbol{v}^{(m)}$ and $\boldsymbol{u}$, we obtain the isomorphism $\Phi_{m}: \psi_{m, 2}^{*} \mathcal{E}^{1} \longrightarrow$ $F$. Then we obtain the sequences of the metrics $\left\{h^{(m)}\right\}$, the connections $\left\{\mathbb{D}^{1(m)}\right\}$, the (non-holomorphic) Higgs fields $\left\{\theta^{(m)}\right\}$ and the conjugates $\left\{\theta^{(m) \dagger}\right\}$. We also obtain the sequences of the holomorphic structures $\bar{\partial}_{F}^{(m)}:=d_{F}^{\prime \prime}-\theta^{(m) \dagger}$.

Theorem 1.9 (Theorem 9.5).

- We can pick a subsequence $\left\{m_{i}\right\}$ of $\{m\}$ such that the corresponding sequences $\left\{h^{\left(m_{i}\right)}\right\},\left\{\mathbb{D}^{1\left(m_{i}\right)}\right\},\left\{\theta^{\left(m_{i}\right)}\right\},\left\{\theta^{\left(m_{i}\right) \dagger}\right\},\left\{\bar{\partial}_{F}^{\left(m_{i}\right)}\right\}$ converge in the $L_{l}^{p}$-sense for any $l$ and for any sufficiently large $p$. The limits are denoted by $h^{(\infty)}, \mathbb{D}^{(\infty)}, \theta^{(\infty)}, \theta^{\dagger(\infty)}$, and $\bar{\partial}_{F}^{(\infty)}$.

- The tuple $\left(F, \bar{\partial}_{F}^{(\infty)}, \theta^{(\infty)}, h^{(\infty)}\right)$ is a CVHS.

\subsection{Mixed twistor structure}

In the previous subsection, we stated the results for the holomorphic flat bundle $\left(\mathcal{E}^{1}, \mathbb{D}^{1}\right)$. In fact, we will consider the $\lambda$-connections $\left(\mathcal{E}^{\lambda}, \mathbb{D}^{\lambda}\right)$, and the conjugates $\left(\mathcal{E}^{\dagger \mu}, \mathbb{D}^{\dagger \mu}\right)$ for all $\lambda$ and $\mu$. We will show similar results, at once. It is the merit to consider all of $\lambda$ and $\mu$ that we obtain a limiting mixed twistor structure, which is a partial solution of a conjecture by Simpson in [36]. (See Definition 2.30 for the definition of mixed twistor.)

Let $\left(E, \bar{\partial}_{E}, \theta, h\right)$ be a tame nilpotent harmonic bundle with trivial parabolic structure over $\Delta^{* 2}$. Let $P$ be a point of $\Delta^{* 2}$, and $O$ be the 
origin of $\Delta^{2}$. We obtain the vector bundle $S(O, P)$ over $\mathbb{P}^{1}$, and the morphisms $N_{i}^{\triangle}: S(O, P) \longrightarrow S(O, P) \otimes \mathcal{O}_{\mathbb{P}^{1}}(2)$ for $i=1,2$. (See Subsubsection 5.2.2 for the construction.) We put $N^{\triangle}(\underline{2}):=\sum_{i=1}^{2} N_{i}^{\triangle}$. We denote the weight filtration of $N^{\triangle}(\underline{2})$ by $W(\underline{2})$.

Theorem 1.10 (A limiting mixed twistor theorem, Theorem 7.2).

- For any neighborhood $U$ of $O$ in $\Delta^{2}$, we can take an appropriate point $P \in U \cap \Delta^{* 2}$ such that the filtered vector bundle $(S(O, P)$, $W(\underline{2}))$ is a mixed twistor.

- The morphisms $N_{i}^{\triangle}$ is a morphism of mixed twistors.

The mixed twistor structure is essentially used in the proof of the compatibility of the filtrations, that is, Theorem 1.6. Briefly speaking, the proof of Theorem 1.6 is divided into three steps:

Step 1 . Let $b$ denote the bottom number of the filtration $W(\underline{1})$. We see that we only have to prove the coincidence in the bottom part, i.e., $W^{(1)}(\underline{2})_{h+b} \cap \mathcal{G} r_{b}^{(1)}=W\left(N^{(1)}(\underline{2})\right)_{h} \cap \mathcal{G} r_{b}^{(1)}$.

Step 2. We see the implication $W^{(1)}(\underline{2})_{h+b} \cap \mathcal{G} r_{b}^{(1)} \supset W\left(N^{(1)}(\underline{2})\right)_{h} \cap$ $\mathcal{G} r_{b}^{(1)}$.

Step 3 . We see the coincidence $W^{(1)}(\underline{2})_{h+b} \cap \mathcal{G} r_{b}^{(1)}=W\left(N^{(1)}(\underline{2})\right)_{h} \cap$ $\mathcal{G} r_{b}^{(1)}$.

Step 1 is rather elementary. We will only use the linear algebra. In Step 2, we use a comparison method and norm estimate in one dimensional case. However we need only rather classical analysis. We use the mixed twistor in Step 3. We need the following:

- The filtration $W(\underline{2})$ of $S(O, P)$ is a mixed twistor structure. This is a consequence of a limiting mixed twistor theorem.

- We have the vector bundle:

$$
\mathcal{G} r_{b, h}^{W\left(N^{(1)}(\underline{2})\right)}:=\frac{\mathcal{G} r_{b}^{(1)} \cap W\left(N^{(1)}(\underline{2})\right)_{h}}{\mathcal{G} r_{b}^{(1)} \cap W\left(N^{(1)}(\underline{2})\right)_{h-1}} .
$$

The equalities $c_{1}\left(\mathcal{G} r_{h, b}^{W\left(N^{(1)}(\underline{2})\right)}\right)=(h+b) \cdot \operatorname{rank}\left(\mathcal{G} r_{h, b}^{W\left(N^{(1)}(\underline{2})\right)}\right)$ hold for any $h$. Here $c_{1}(\mathcal{F})$ denotes the first Chern class of a coherent sheaf $\mathcal{F}$ on $\mathbb{P}^{1}$. 
From these two facts and the implication

$$
W^{(1)}(\underline{2})_{h+b} \cap \mathcal{G} r_{b}^{(1)} \subset W\left(N^{(1)}(\underline{2})\right)_{h} \cap \mathcal{G} r_{b}^{(1)},
$$

we obtain the coincidence, due to some general lemma for mixed twistors.

However, the consideration of all $\left(\mathcal{E}^{\lambda}, \mathbb{D}^{\lambda}\right)$ and $\left(\mathcal{E}^{\dagger \mu}, \mathbb{D}^{\dagger \mu}\right)$ raises some difficulties. It is a principle that the arguments over $\mathbf{C}_{\lambda}^{*}$ are not different from the arguments for $\lambda=1$. On the other hand, we need some additional argument around $\lambda=0$.

One big difference is the existence of normalizing frames. Let $\boldsymbol{v}$ be a holomorphic frame of $\mathcal{E}^{\lambda}$ over $X-D$. We obtain the $\lambda$-connection form $\mathcal{A}=\sum A_{j} \cdot d z_{j} / z_{j} \in \Gamma\left(X-D, M(r) \otimes \Omega_{X-D}^{1,0}\right)$, determined by the relation $\mathbb{D} \boldsymbol{v}=\boldsymbol{v} \cdot \mathcal{A}$. If $A_{j}$ are constant, the frame $\boldsymbol{v}$ is called a normalizing frame. When $\lambda \neq 0$, we can always take a normalizing frame. On the contrary, we do not have a normalizing frame in general, in the case $\lambda=0$.

We will see that ${ }^{\diamond} \mathcal{E}^{\lambda}$ is locally free. Simpson has already shown that ${ }^{\diamond} \mathcal{E}^{\lambda}$ is locally free for all $\lambda$, if the base manifold is one dimensional. By using the fact, it is easy to see that the normalizing frame of the prolongment $\mathcal{E}^{\lambda}$ gives, in fact, the frame of ${ }^{\diamond} \mathcal{E}^{\lambda}$. However, we have to prove some extension property of holomorphic sections on hyperplanes, if $\lambda=0$.

One more point which we should care is the conjugacy classes of the residues. When the base manifold is one dimensional, Simpson showed that the conjugacy classes of $N_{\mid(\lambda, O)}$ are independent of $\lambda$. Thus it is

easy to see that the conjugacy classes of $N_{1 \mid(\lambda, Q)}$ are independent of $\lambda$, when $Q$ is contained in $D_{1}-D_{2}$. On the other hand, it is easy to see the conjugacy classes of $N_{1 \mid(\lambda, Q)}$ are independent of $Q$ due to the existence of a normalizing frame, when we fix $\lambda \neq 0$. As a result, we can immediately obtain that the conjugacy classes of $N_{1 \mid(\lambda, Q)}$ are independent of $(\lambda, Q) \in \mathbf{C}_{\lambda} \times D_{\underline{1}}-\{0\} \times D_{\underline{2}}$. However we need some argument to see that the degeneration of the conjugacy classes does not occur at $\{0\} \times D_{2}$. Interestingly, we can show that the conjugacy classes of $N_{1 \mid(\lambda, Q)}$ are independent of $\lambda$ for any $Q \in D_{\underline{1}}$, by using a limiting mixed twistor theorem.

\subsection{Prolongment of Higgs bundle}

Let $\left(E, \bar{\partial}_{E}, \theta, h\right)$ be a tame nilpotent harmonic bundle with trivial parabolic structure on $X-D$ as in Subsubsection 1.2.1. 
Let ${ }^{\diamond} \mathcal{E}^{0}$ denote the prolongment of the holomorphic bundle $\mathcal{E}^{0}=$ $\left(E, \bar{\partial}_{E}\right)$ by an increasing order (See Subsection 4.1).

Theorem 1.11 (Theorem 4.1). The sheaf ${ }^{\diamond} \mathcal{E}^{0}$ is coherent and locally free.

In the case of variation of Hodge structures, this is a consequence of nilpotent orbit theorem due to $\operatorname{Schmid}([32])$. Let $(V, \mathcal{F},\langle\cdot, \cdot\rangle)$ be a polarized variation of Hodge structures over $X-D$. Here $V$ is $\mathbf{R}$-local system, $\mathcal{F}$ be the Hodge filtration, and $\langle\cdot, \cdot\rangle$ be the polarization. For simplicity, we assume the monodromies are unipotent. The holomorphic bundle $\mathcal{E}^{1}=V \otimes_{\mathbf{R}} \mathcal{O}_{X-D}$ is canonically extended to the holomorphic bundle ${ }^{\diamond} \mathcal{E}^{1}$ with the regular connection over $X$. Due to the nilpotent orbit theorem, $\mathcal{F}$ is extended to the filtration ${ }^{\diamond} \mathcal{F}$ of ${ }^{\diamond} \mathcal{E}^{1}$. In our terminology, we have the following:

$$
\mathcal{E}^{0}=\operatorname{Gr}^{\mathcal{F}}\left(\mathcal{E}^{1}\right), \quad{ }^{0} \mathcal{E}^{0}=\operatorname{Gr}^{\diamond \mathcal{F}}\left({ }^{\diamond} \mathcal{E}^{1}\right)
$$

\subsection{Outline of the paper}

\subsubsection{Section 2}

In Subsections 2.1-2.4, we make a preparation on the commuting tuples of nilpotent maps and the weight filtrations, on vector spaces or vector bundles. We explain some of terminology. We also discuss the compatibilities of several filtrations and nilpotent maps. What we would like to see is summarized in Corollary 2.2, Proposition 2.1 and Corollary 2.3. In Subsection 2.5, we recall mixed twistor structure and give lemmas which will be used later. The author feels that the mixed twistor is useful when we would like to obtain a lower bound of the degree with respect to a filtration.

\subsubsection{Section 3}

In Subsection 3.1, we recall the definition of harmonic bundles. Following Simpson ([36] and [37]), we consider the deformed holomorphic bundle and the conjugate. In Subsection 3.2, we recall some easy examples of harmonic bundles over the punctured disc $\Delta^{*}$. In particular, the model bundles $\operatorname{Mod}(l, a, C)$ will be used later as a convenient tool. These examples can be closely investigated by direct calculations. Some results, for example, the corollaries $3.1,3.2,3.3,3.4$ and 3.5 will be

used. In Subsection 3.3, we discuss the 'convergency' of a sequence of 
the harmonic bundles. We will see that we can pick a nice 'convergent' subsequence, as is naturally expected from the ellipticity of harmonic bundles.

\subsubsection{Section 4}

In Subsection 4.1, we prepare some words used for prolongments of vector bundles. In Subsection 4.2, we recall definitions of tameness and nilpotentness. We will recall an estimate of the norms of Higgs fields with respect to the metric. In Subsection 4.3, we will recall some results of Simpson in one dimensional case. In Subsection 4.4, we will recall the definition of triviality of parabolic structures. We will also see that the tame nilpotent rank one harmonic bundle with trivial parabolic structure is smooth.

After Subsection 4.4, the harmonic bundles will be always assumed to be tame, nilpotent and has trivial parabolic structure.

In the remaining of the Section 4, we will see that the prolongment ${ }^{\diamond} \mathcal{E}$ of the deformed holomorphic bundle by an increasing order are locally free. We will use some ideas of Cornalba-Griffiths ([11]). In Subsection 4.5, we recall something from their paper. In Subsubsections 4.6.1-4.6.2, a normalizing frame gives a frame over $\mathcal{X}^{\sharp}$, and we show that ${ }^{\diamond} \mathcal{E}$ is locally free if ${ }^{0} \mathcal{E}^{0}$ is locally free. In particular, it implies that the ${ }^{\diamond} \mathcal{E}$ is locally free when the base manifold is one dimensional. In Subsubsection 4.6.3, we state the family versions of the results in Subsection 4.3. In Subsection 4.7, we will show the extendability of the sections on hyperplane in the case $\lambda=0$. It immediately implies that $\diamond^{0}$ is locally free, and thus that the ${ }^{\diamond} \mathcal{E}$ is locally free. In Subsection 4.8, we see the functoriality of the prolongment.

\subsubsection{Section 5}

In Subsections 5.1-5.2, we recall the construction of the vector bundle $S(Q, P)$ of Simpson. In Subsection 5.3, a limiting mixed twistor theorem is given and proved, in the one dimensional case. In Subsubsection 5.4.1, a refinement for higher dimensional case is given. Once we obtain a mixed twistor structure, briefly speaking, we know that 'a degeneration at $\lambda=0$ does not occur'. Some easy and useful consequences of such type are given in Subsubsections 5.4.2 and 5.4.3. In Subsubsection 5.4.4, we give a weak constantness of the filtrations as an easy consequence of a limiting mixed twistor structure, although it can be shown without 
mixed twistor structure.

\subsubsection{Section 6}

In Subsection 6.1, we explain our method of comparison to obtain some estimate for metrics. The method will be used in the beginning of all of the latter sections. Briefly speaking, the method reduces the estimate over the region to the estimate over the boundary. Since the dimension of the boundary is lower, we can use an estimate for lower dimensional case. (In Subsection 6.1, we only need a rough estimate on the boundary.) In Subsection 6.2, we consider the morphisms $\psi_{m, \underline{1}}: \Delta^{n} \longrightarrow \Delta^{n}$ defined by $\left(z_{1}, \ldots, z_{n}\right) \longmapsto\left(z_{1}^{m}, z_{2}, \ldots, z_{n}\right)$. For a harmonic bundle $\left(E, \bar{\partial}_{E}, \theta, h\right)$ over $\Delta^{* l} \times \Delta^{n-l}$, we obtain the sequence $\left\{\psi_{m, \underline{1}}^{*}\left(E, \bar{\partial}_{E}, \theta, h\right)\right\}$ of harmonic bundles. We can apply the result of Subsection 3.3, due to the rough norm estimate obtained in Subsection 6.1. In Subsection 6.3 , we see some orthogonality in the limit. In particular, we see that the limiting harmonic bundle is a CVHS in one dimensional case. In Subsection 6.4, we investigate the first Chern class of the vector bundle $\mathcal{G} r_{h_{1}, h_{2}}^{W\left(N^{\Delta(1)}(2)\right)}$ over $\mathbb{P}^{1}$, obtained in Subsubsection 5.4.3.

\subsubsection{Section 7}

In Section 7, we will prove the constantness of the filtrations on the positive cones for the tuple of residues of harmonic bundles. As a preliminary, we give a norm estimate in some special case in Subsection 7.1. Then we consider the morphisms $\psi_{m, n}: \Delta^{n} \longrightarrow \Delta^{n}$ defined by $\left(z_{1}, \ldots, z_{n}\right) \longmapsto\left(z_{1}^{m}, \ldots, z_{n}^{m}\right)$ in Subsection 7.2. By investigating the limiting harmonic bundle, we obtain the constantness of the filtrations on the positive cones in some special case. Although the theorem is stated in Subsection 7.3, the main part of the proof is done in Subsection 7.2 .

\subsubsection{Section 8}

In Section 8, we see the strongly sequential compatibility of the residues. In Subsubsection 8.1.1, we see some compatibility in the bottom part in the two dimensional case. By using a method of comparison, we obtain some implication of the filtrations. Then we obtain the coincidence by using the result in Subsubsection 5.4.3 and Lemma 2.19 for mixed twistor structures. Once we know such compatibility in two dimensional case, a similar compatibility in the higher dimensional case is easy to 
obtain, which is shown in Subsubsection 8.1.2. Then we obtain the theorems in Subsection 8.2.

\subsubsection{Section 9}

In Subsection 9.1, we obtain a norm estimate. As a preliminary, we consider the pull back of harmonic bundle $\left(E, \bar{\partial}_{E}, \theta, h\right)$ via the 'blowup' $\phi_{N}: \widetilde{X} \longrightarrow X$, and we obtain the norm estimate for $\phi_{N}^{*}\left(E, \bar{\partial}_{E}, \theta, h\right)$ in Subsubsection 9.1.1. The method is same as that in 6.1. Since we have already shown the strongly sequential compatibility of the residues in Subsection 8.2, we can obtain a stronger estimate. By translating such a result, we obtain the theorem in Subsubsection 9.1.3. In Subsubsection 9.2.1, we consider the pull backs of the harmonic bundles via the morphism $\psi_{m, \underline{n}}$, as in Subsection 7.2. Then we obtain a limiting harmonic bundle. We see that it is, in fact, a CVHS. As an application of limiting CVHS, we see the purity theorem in Subsubsection 9.2.3.

\subsection{Some remarks}

Unfortunately, this paper looks rather long. However, the reader will know that much of the part is elementary and not new for both of the reader and the author. Many of the definitions, the lemmas and the propositions are more or less standard, familiar and obvious. They are included to clarify what the author would like to say.

This paper is the revision of [29]. The main difference is as follows:

- In [29], the dimensions of the base manifolds are assumed to be less than two. In this paper, we discuss higher dimensional case. The much part of the preliminary for the filtrations is added for that purpose.

- The explanation for the norm estimate in one dimensional case is added. In [35], such estimates are proven for the cases $\lambda=0$ and $\lambda=1$. Clearly, Simpson's argument works for the other $\lambda$. We only indicate how to change.

- The explanation for the prolongation of the deformed holomorphic bundle is added.

- The mixed twistor structure is used more efficiently. As a result, some arguments for the filtrations on the divisors are simplified. 
- The author hopes that the discussion and the explanation in this paper are clearer than those in the previous version [29].

The author's original motivation of the study is to generalize the Kobayashi-Hitchin correspondence (see for example, [4], [14], [34], [35] and [39]). Namely he would like to clarify the relation of stable Higgs bundles and harmonic bundles over a quasi projective variety. For that purpose it seems important to characterize the residues of the Higgs fields. Unfortunately, the understanding seems insufficient to solve such problem in this stage, for the author. Probably, one direction of the study is a more precise comparison of a limiting CVHS and the original harmonic bundle.

\subsection{Acknowledgement}

The author sincerely thanks the referee for his substantial effort to read the paper and his very useful suggestions. He kindly informed on the paper of Jost-Li-Zuo [21]. He also informed his alternative proof of local freeness of $\diamond \mathcal{E}^{0}$.

The author is grateful to the colleagues in Osaka City University. In particular, he thanks Mikiya Masuda for his encouragement. The author expresses his gratitude to Akira Ishii and Yoshifumi Tsuchimoto for their encouragements.

The author thanks the financial supports by Japan Society for the Promotion of Science and the Sumitomo Foundation. The paper was written during his stay at the Institute for Advanced Study. The author is sincerely grateful to their excellent hospitality. He also acknowledges National Scientific Foundation for a grant DMS 9729992, although any opinions, findings and conclusions or recommendations expressed in this material are those of the author.

\subsection{Some sets}

We will use the following notation:

$\mathbb{Z}$ : the set of the integers,

$\mathbb{Z}_{>0}$ : the set of the positive integers,

Q: $\quad$ the set of the rational numbers,

$\mathbf{Q}_{>0}$ : the set of the positive rational numbers,

$\mathbf{R}$ : the set of the real numbers,

$\mathbf{R}_{>0}$ : the set of the positive real numbers,

C: $\quad$ the set of the complex numbers,

$\underline{n}: \quad$ the set $\{1,2, \ldots, n\}$,

$\mathfrak{S}_{l}: \quad$ the $l$-th symmetric group. 
We put as follows:

$$
\begin{gathered}
\Delta(C):=\{z \in \mathbf{C}|| z \mid<C\}, \quad \Delta^{*}(C):=\{z \in \mathbf{C}|0<| z \mid<C\}, \\
\mathbf{C}^{*}=\{z \in \mathbf{C} \mid z \neq 0\} .
\end{gathered}
$$

When $C=1$, we often omit to denote $C$, i.e., $\Delta=\Delta(1)$ and $\Delta^{*}=$ $\Delta^{*}(1)$. If we emphasize the variable, we describe as $\Delta_{z}, \Delta_{i}$. For example, $\Delta_{z} \times \Delta_{w}=\{(z, w) \in \Delta \times \Delta\}$, and $\Delta_{1} \times \Delta_{2}=\left\{\left(z_{1}, z_{2}\right) \in \Delta \times \Delta\right\}$. We often use the notation $\mathbf{C}_{\lambda}$ and $\mathbf{C}_{\mu}$.

Unfortunately, the notation $\Delta$ is also used to denote the Laplacian. The author hopes that there will be no confusion.

The set of $r \times r$-matrices with $\mathbf{C}$-coefficient is denoted by $M(r)$, and the set of $r \times r$-hermitian matrices by $\mathcal{H}(r)$.

In general, $q_{i}: X^{n} \longrightarrow X$ denotes the projection onto the $i$-th component, and $\pi_{i}: X^{n} \longrightarrow X^{n-1}$ denotes the projection omitting the $i$-th component. However we will often use $\pi$ to denote some other projections.

Let $l$ be a positive integer. We have the decomposition of $\mathbf{R}_{\geq 0}^{l}$ into $\coprod_{I \subset \underline{l}} \mathbf{R}_{>0}^{I}$, defined as follows:

$$
\mathbf{R}_{>0}^{I}:=\left\{\left(a_{1}, \ldots, a_{l}\right) \in \mathbf{R}_{\geq 0}^{l} \mid a_{i}>0 \Longleftrightarrow i \in I\right\}
$$

Let $l$ be a positive integer, $C$ be a positive real number, and $\sigma$ be an element of $\mathfrak{S}_{l}$. Then we put as follows:

$$
\begin{aligned}
Z(\sigma, l, C):=\left\{\left(z_{1}, \ldots, z_{n}\right) \in \Delta^{* l} \times \Delta^{n-l} \mid\right. & \\
& \left.\left|z_{\sigma(i-1)}\right|^{C}<\left|z_{\sigma(i)}\right| i=2, \ldots, l\right\} .
\end{aligned}
$$

\subsection{Index of notation}

The following notations or words are defined in the pages refered:

\section{Section 2}

$H(h, \boldsymbol{v}), 368, \quad b(W)$, bottom number, 368,

$W^{(m)}(\underline{j}), 369, \quad N^{\otimes h}, N^{\operatorname{sym} h}, N^{\wedge h}, 373$,

$W(N), 373, \quad W(I), 376$,

adapted, 386 . 


\section{Section 3}

$$
\begin{array}{ll}
R\left(\partial_{E}+\bar{\partial}_{E}\right), R(h), 401, & \theta^{\dagger}, 401, \\
\mathbb{D}^{1}, 401, & \mathcal{X}, 402, \\
\mathcal{E}, 402, & \mathcal{X}^{\lambda}, \mathcal{X} \sharp, 402, \\
\mathcal{E}^{\lambda}, \mathcal{E}^{\sharp}, 402, & \mathbb{D}^{\lambda}, \mathbb{D}, 403, \\
\mathbb{D}^{\lambda, f}, 403, \mathbb{D}^{f}, 404, & X^{\dagger}, 404, \\
\mathcal{X}^{\dagger}, 404, & \mathcal{E}^{\dagger}, 404, \\
\mathcal{X}^{\dagger \mu}, \mathcal{X}^{\dagger \sharp}, 404, & \mathcal{E}^{\dagger}, \mathcal{E}^{\dagger \sharp}, 404, \\
\mathbb{D}^{\dagger \mu}, 404, \mathbb{D}^{\dagger}, \mathbb{D}^{\dagger f}, 405, & \boldsymbol{v}^{\dagger}, 405, \\
L(a), 408, & L(\alpha), 410, \\
\operatorname{Mod}(2), 412, & \operatorname{Mod}(l), 413, \\
\operatorname{Mod}(l, a, C), 413, & E(V, N), 413, \\
\mathcal{M} \operatorname{Mod}(l+1, a, C), 415 . &
\end{array}
$$

\section{Section 4}

$$
\begin{array}{ll}
{ }^{\diamond} E, 433, & (\cdot, \cdot)_{h, g},\langle\cdot, \cdot\rangle_{h},\|\cdot\|_{h}, 451, \\
A_{h}^{p, q}, 451, & \langle\langle\cdot, \cdot\rangle\rangle_{h}, 452, \\
\tau(\boldsymbol{a}, N), h_{\boldsymbol{a}, N}, 454, & \left.|\cdot|\right|_{\boldsymbol{a}, N},\|\cdot\|_{\boldsymbol{a}, N},(\cdot, \cdot)_{\boldsymbol{a}, N},\langle\langle\cdot, \cdot\rangle\rangle_{\boldsymbol{a}, N}, 454, \\
A_{\boldsymbol{a}, N}^{p, q}(E), 454, & \Omega(\boldsymbol{v}), 458 .
\end{array}
$$

\section{Section 5}

$$
S(Q, P), 481, \quad \mathcal{N}_{i}^{\triangle}, 482, \quad W^{\triangle}, 484 .
$$

\section{Section 6}

$$
\mathcal{G} r_{\left(h_{1}, h_{2}\right)}^{W\left(N^{\triangle(1)}(2)\right)}, 501 \text {. }
$$

\section{Preliminary for filtrations}

\subsection{Vector space and filtrations}

\subsubsection{Base and metric}

Let $V$ be an $n$-dimensional vector space over $\mathbf{C}$. To describe a base of $V$, or more generally, to describe a tuple of elements of $V$, we use a notation $\boldsymbol{v}=\left(v_{1}, \ldots, v_{n}\right)$. Let $\boldsymbol{v}$ and $\boldsymbol{w}$ be two bases of $V$. We obtain the matrix $A=\left(A_{i j}\right)$ determined by the following formula:

$$
v_{j}=\sum_{i} A_{i j} \cdot w_{i}
$$

In that case, it is described as $\boldsymbol{v}=\boldsymbol{w} \cdot A$. 
Let $h$ be a hermitian metric of $V$. Then we have the hermitian matrix $H(h, \boldsymbol{v})=\left(H_{i j}\right)$ determined as follows:

$$
H_{i j}:=h\left(v_{i}, v_{j}\right) .
$$

The $H(h, \boldsymbol{v}) \in \mathcal{H}(n)$ is called the hermitian matrix of the metric $h$ with respect to $\boldsymbol{v}$.

\subsubsection{Compatibility with direct sum}

Let $V$ be a finite dimensional vector space with a direct sum decomposition $V=\bigoplus_{i} V_{i}$.

Let $v$ be a nonzero element of $V$. It is called compatible with the decomposition if there exists an $i$ such that $v \in V_{i}$. The number $i$ is called the degree of $v$.

Let $\boldsymbol{v}=\left(v_{1}, \ldots, v_{n}\right)$ be a base of $V$. It is compatible with the decomposition if each $v_{i}$ is compatible with the decomposition.

Let $W$ be an increasing filtration of $V$. It is called compatible with the decomposition if $W_{j}=\bigoplus_{i}\left(W_{j} \cap V_{i}\right)$ for any $j$. We denote the induced filtration of $V_{i}$ by $W \cap V_{i}$.

Let $f$ be an endomorphism of $V$. It is called compatible with the decomposition if $f\left(V_{i}\right) \subset V_{i}$.

For a tuple $\left(u_{1}, \ldots, u_{l}\right)$ of elements of $V,\left\langle u_{1}, \ldots, u_{l}\right\rangle$ denotes the vector subspace generated by $\left(u_{1}, \ldots, u_{l}\right)$.

\subsubsection{Filtration}

In this paper, we mainly use increasing filtrations. Thus 'filtration' means an 'increasing filtration' if we do not notice.

Let $V$ be a vector space with a filtration $W$, the associated graded vector space is denoted by $G r^{W}=\bigoplus_{i} G r_{i}^{W}$, where $G r_{i}^{W}:=W_{i} / W_{i-1}$.

For a filtration $W$, we have the number $b(W)$ determined as follows:

$$
b(W):=\min \left\{h \mid G r_{h}^{W} \neq 0\right\} .
$$

The number $b(W)$ is called the bottom number of $W$.

For a nonzero element $v \in V$, the number $\operatorname{deg}^{W}(v)$ is defined as follows:

$$
\operatorname{deg}^{W}(v):=\min \left\{h \mid v \in W_{h}\right\} .
$$

The number $\operatorname{deg}^{W}(v)$ is called the degree of $v$ with respect to the filtration $W$. We have the induced element $v^{(1)}$ of $G r_{\operatorname{deg}^{W}(v)}^{W}(V)$. 
Let $\boldsymbol{v}=\left(v_{1}, \ldots, v_{n}\right)$ be a base of $V$. We say that $\boldsymbol{v}$ is compatible with the filtration $W$, if the following is satisfied:

For any $i$, we have a subset $I \subset\{1, \ldots, n\}$ such that $\left\{v_{j} \mid j \in\right.$ $I\}$ gives a base of $W_{i}$.

In that case, the induced elements $\boldsymbol{v}^{(1)}=\left\{v_{1}^{(1)}, \ldots, v_{n}^{(1)}\right\}$ gives a base of $G r^{W}(V)$ compatible with the natural decomposition.

An endomorphism $f$ of $V$ is called compatible with the filtration $W$ if $f\left(W_{i}\right) \subset W_{i}$.

\subsubsection{The induced filtrations}

Let $W(\underline{1})$ and $W(\underline{2})$ be filtrations on $V$. We have the associated graded space of $W(\underline{1})$ :

$$
G r^{(1)}:=\bigoplus_{a} G r_{a}^{(1)}, \quad G r_{a}^{(1)}:=G r_{a}^{W(\underline{1})}
$$

We have the induced filtration $W^{(1)}(\underline{2})$ by $W(\underline{2})$ on $G r^{(1)}$, which is defined as follows:

$$
\begin{aligned}
W^{(1)}(\underline{2})_{l} & :=\bigoplus_{a} W^{(1)}(\underline{2})_{l} \cap G r_{a}^{(1)}, \\
W^{(1)}(\underline{2})_{l} \cap G r_{a}^{(1)} & :=\frac{W(\underline{2})_{l} \cap W(\underline{1})_{a}}{W(\underline{1})_{a-1}} \subset G r_{a}^{(1)} .
\end{aligned}
$$

Let $(W(\underline{1}), W(\underline{2}), \ldots, W(\underline{n}))$ be filtrations on $V$. We have the induced filtrations $\left(W^{(1)}(\underline{2}), \ldots, W^{(1)}(\underline{n})\right)$ on $G r^{(1)}$. Inductively, we obtain the filtrations $W^{(m)}(j)$ on $G r^{(m)}$ for $1 \leq m<j \leq n$ as follows:

1. On $G r^{(m)}$, we have the filtrations $W^{(m)}(\underline{j})$ for $(j=m+1, \ldots, n)$.

2. Then we put $G r^{(m+1)}:=G r^{W^{(m)}} \underline{(m+1)}$. We have the filtrations $W^{(m+1)}(j)(j=m+2, \ldots, n)$ induced by $W^{(m)}(\underline{j})$.

\subsubsection{Compatible sequence of filtrations}

Let $(W(\underline{1}), W(\underline{2}), \ldots, W(\underline{n}))$ be filtrations on $V$. Let $\boldsymbol{h}=\left(h_{1}, \ldots, h_{n}\right)$ be a tuple of integers. We have the following morphism:

$$
\pi_{\boldsymbol{h}}: \bigcap_{j=1}^{n} W(\underline{j})_{h_{j}} \longrightarrow G r_{h_{1}}^{(1)}
$$

The image of $\pi_{\boldsymbol{h}}$ is always contained in $G r_{h_{1}}^{(1)} \cap \bigcap_{j=2}^{n} W^{(1)}(\underline{j})_{h_{j}}$. 
Definition 2.1. A sequence of filtrations $(W(\underline{1}), \ldots, W(\underline{n}))$ is called compatible if the following holds, inductively:

1. $\left(W^{(1)}(\underline{2}), \ldots, W^{(1)}(\underline{n})\right)$ is a compatible sequence.

2. For any $\boldsymbol{h} \in \mathbb{Z}^{n}$, the image of $\pi_{\boldsymbol{h}}$ is same as $G r_{h_{1}}^{(1)} \cap \bigcap_{j=2}^{n} W^{(1)}(\underline{j})_{h_{j}}$.

Remark 2.1. When $n \leq 2$, the condition is trivial.

Definition 2.2. Let $(W(\underline{1}), \ldots, W(\underline{n}))$ be a compatible sequence of filtrations. A nonzero element $f \in V$ is called compatible with the sequence $(W(\underline{1}), \ldots, W(\underline{n}))$ if the following holds, inductively:

1. The induced element $f^{(1)} \in G r^{(1)}$ is compatible with the sequence $\left(W^{(1)}(\underline{2}), \ldots, W^{(1)}(\underline{n})\right)$.

2. For any $j \geq 2$, we have $\operatorname{deg} W(\underline{j})(f)=\operatorname{deg}^{W^{(1)}}(\underline{j})\left(f^{(1)}\right)$.

Definition 2.3. Let $(W(\underline{1}), \ldots, W(\underline{n}))$ be a compatible sequence of filtrations. A base $\boldsymbol{v}=\left(v_{i}\right)$ of $V$ is called compatible, if the following holds, inductively:

- For each $i$, the element $v_{i}$ is compatible with the sequence $(W(\underline{1})$, $\ldots, W(\underline{n}))$.

- $\boldsymbol{v}$ is a compatible base with $W(\underline{1})$.

- The induced base $\boldsymbol{v}^{(1)}$ of $G r^{(1)}$ is compatible with the sequence $\left(W^{(1)}(\underline{2}), \ldots, W^{(1)}(\underline{n})\right)$.

Consider a decomposition of $V$ into $\bigoplus_{h \in \mathbb{Z}^{n}} U_{\boldsymbol{h}}$.

Definition 2.4. Let $(W(\underline{1}), \ldots, W(\underline{n}))$ be a compatible sequence of filtrations. A decomposition $V=\bigoplus_{h \in \mathbb{Z}^{n}} U_{h}$ is called a splitting compatible with the sequence $(W(\underline{1}), \ldots, W(\underline{n}))$, if the following holds for any $\boldsymbol{h} \in \mathbb{Z}^{n}$ :

$$
\bigcap_{j=1}^{n} W(\underline{1})_{q_{j}(\boldsymbol{h})}=\bigoplus_{\boldsymbol{k} \in \mathcal{T}(\boldsymbol{h})} U_{\boldsymbol{k}}, \quad \mathcal{T}(\boldsymbol{h}):=\left\{\boldsymbol{k} \in \mathbb{Z}^{n} \mid q_{j}(\boldsymbol{k}) \leq q_{j}(\boldsymbol{h})\right\} .
$$

Here $q_{j}$ denotes the projection $\mathbb{Z}^{n} \longrightarrow \mathbb{Z}$ onto the $j$-th component.

The dimension of $U_{\boldsymbol{h}}$ is denoted by $d(\boldsymbol{h})$.

Lemma 2.1. Let $(W(\underline{1}), \ldots, W(\underline{n}))$ be a compatible sequence of filtrations. 
1. Let $\boldsymbol{v}$ be a base of $V$ compatible with the decomposition $V=$ $\bigoplus_{\boldsymbol{h} \in \mathbb{Z}^{n}} U_{\boldsymbol{h}}$. Assume that the decomposition is compatible with $(W(\underline{1}), \ldots, W(\underline{n}))$. Then the base $\boldsymbol{v}$ is compatible with $(W(\underline{1}), \ldots$, $W(\underline{n}))$.

2. Let $\boldsymbol{v}$ be a base of $V$ compatible with the sequence $(W(\underline{1}), \ldots$, $W(\underline{n}))$. We put as follows:

$$
U_{\boldsymbol{h}}:=\left\langle v_{i} \mid \operatorname{deg}^{W(\underline{j})}\left(v_{i}\right)=q_{j}(\boldsymbol{h})\right\rangle .
$$

Then the decomposition $V=\bigoplus_{\boldsymbol{h} \in \mathbb{Z}^{n}} U_{\boldsymbol{h}}$ is compatible with the sequence $(W(\underline{1}), \ldots, W(\underline{n}))$.

Proof. Consider the first claim. We use an induction on $n$. Let $\boldsymbol{v}$ be a base compatible with the decomposition. Clearly it is compatible with the filtration $W(\underline{1})$.

Let $\pi_{h}^{\prime}$ denote the composite of the following morphisms. Here we put $h_{1}=h$ for simplicity of notation:

$$
\bigoplus_{\substack{\boldsymbol{k} \in \mathbb{Z}^{n} \\ q_{1}(\boldsymbol{k})=h}} U_{\boldsymbol{k}} \stackrel{\subset}{\longrightarrow} \bigcap_{j=1}^{n} W(\underline{j})_{h_{j}} \stackrel{\pi_{h}}{\longrightarrow} G r_{h}^{(1)} .
$$

Since the decomposition is compatible with the sequence of the filtrations, $\pi_{h}^{\prime}$ is isomorphic. Thus we obtain the decomposition of $G r_{h}^{(1)}$ as follows:

$$
G r_{h}^{(1)}=\bigoplus_{\substack{\boldsymbol{k} \in \mathbb{Z}^{n} \\ q_{1}(\boldsymbol{k})=h}} \pi_{h}^{\prime}\left(U_{\boldsymbol{k}}\right)
$$

Due to the following isomorphism, the decomposition is compatible with the sequence $\left(W^{(1)}(\underline{2}), \ldots, W^{(1)}(\underline{n})\right)$ :

$$
\begin{aligned}
\bigoplus_{\boldsymbol{k}^{\prime} \in \mathcal{T}(l)} \pi_{h}^{\prime}\left(U_{\left(h, \boldsymbol{k}^{\prime}\right)}\right) & \simeq \frac{W(\underline{1})_{h} \cap \bigcap_{j=1}^{n-1} W(\underline{j+1})_{l_{j}}}{W(\underline{1})_{h-1} \cap \bigcap_{j=1}^{n-1} W(\underline{j+1})_{l_{j}}} \\
& \simeq G r_{h}^{(1)} \cap \bigcap_{j=1}^{n-1} W^{(1)}(\underline{j+1})_{l_{j}},
\end{aligned}
$$

Here we put $\mathcal{T}(\boldsymbol{l})=\left\{\boldsymbol{k}^{\prime} \in \mathbb{Z}^{n-1}, q_{j}\left(\boldsymbol{k}^{\prime}\right) \leq l_{j}, j=1, \ldots n-1\right\}$, and $\left(h, \boldsymbol{k}^{\prime}\right)$ denotes $\left(h, k_{1}^{\prime}, \ldots, k_{n-1}^{\prime}\right)$ for $\boldsymbol{k}^{\prime}=\left(k_{1}^{\prime}, \ldots, k_{n-1}^{\prime}\right)$. By our assumption of 
the induction, the induced base $\boldsymbol{v}^{(1)}$ is compatible with the sequence $\left(W^{(1)}(\underline{2}), \ldots, W^{(1)}(\underline{n})\right)$. We also have $\operatorname{deg}^{W(\underline{j})}\left(v_{i}\right)=\operatorname{deg}^{W^{(1)}(\underline{j})}\left(v_{i}\right)$ for $j \geq 2$. Thus we obtain the first claim.

The second claim can be shown similarly.

q.e.d.

Lemma 2.2. Let $(W(\underline{1}), \ldots, W(\underline{n}))$ be a compatible sequence of the filtrations. There exists a decomposition $V=\bigoplus_{h \in \mathbb{Z}^{n}} U_{\boldsymbol{h}}$ compatible with the sequence $(W(\underline{1}), \ldots, W(\underline{n}))$.

Proof. We use an induction on $n$. Consider the induced filtrations $G r_{h}^{(1)} \cap W^{(1)}(j)$ on $G r_{h}^{(1)}$, which is easily checked to be compatible. By our assumption of the induction, we can take a compatible decomposition:

$$
G r_{h}^{(1)}=\bigoplus_{\boldsymbol{k} \in \mathbb{Z}^{n-1}} U_{h, \boldsymbol{k}}^{\prime}, \quad G r_{h}^{(1)} \cap \bigcap_{j=1}^{n-1} W^{(1)}(\underline{j+1})_{l_{j}}=\bigoplus_{\boldsymbol{k} \in \mathcal{T}(\boldsymbol{l})} U_{h, \boldsymbol{k}}^{\prime} .
$$

For an element $\boldsymbol{h}=\left(h_{1}, \ldots, h_{n}\right) \in \mathbb{Z}^{n}$, we put $\boldsymbol{h}^{\prime}=\left(h_{2}, \ldots, h_{n}\right)$. Since $(W(\underline{1}), \ldots, W(\underline{n}))$ is compatible, $U_{h_{1}, h^{\prime}}^{\prime}$ is contained in the following morphism:

$$
\pi_{\boldsymbol{h}}: W(\underline{1})_{h_{1}} \cap \bigcap_{j=2}^{n} W(\underline{j})_{h_{j}} \longrightarrow G r_{h_{1}}^{(1)} \cap \bigcap_{j=2}^{n} W^{(1)}(\underline{j})_{h_{j}} .
$$

We pick the subspace $U_{\boldsymbol{h}}$ of $W(\underline{1})_{h_{1}} \cap \bigcap_{j=2}^{n} W(\underline{j})_{h_{j}}$, which is isomorphic to $U_{h_{1}, h^{\prime}}^{\prime}$ via the morphism $\pi_{h}$. Then we obtain the decomposition $V=\bigoplus_{h \in \mathbb{Z}^{n}} U_{h}$.

By our construction, we have the following:

$$
\left[W(\underline{1})_{h_{1}} \cap \bigcap_{j=2}^{n} W(\underline{j})_{h_{j}}\right]=\left[W(\underline{1})_{h_{1}-1} \cap \bigcap_{j=2}^{n} W(\underline{j})_{h_{j}}\right] \oplus\left[\underset{\boldsymbol{k} \in \mathcal{T}\left(\boldsymbol{h}^{\prime}\right)}{\bigoplus} U_{\left(h_{1}, \boldsymbol{k}\right)}\right] .
$$

Thus the induction can proceed.

q.e.d.

\subsection{A commuting tuple of nilpotent maps}

\subsubsection{Tensor product, symmetric products and exterior prod- ucts}

Let $V$ be a finite dimensional vector space over $\mathbf{C}$ and $N$ be an endomorphism of $V$. Then we have the tensor product $V^{\otimes h}$, the symmetric 
products $\operatorname{Sym}^{h}(V)$ and the exterior products $\bigwedge^{h}(V)$. We also have the endomorphism of $V^{\otimes h}$ :

$$
\widetilde{N}:=\sum \overbrace{1 \otimes \cdots \otimes 1}^{j-1} \otimes N \otimes \overbrace{1 \otimes \cdots \otimes 1}^{h-j} .
$$

We often denote $\tilde{N}$ by $N^{\otimes n}$. We will not use the endomorphism $\overbrace{N \otimes \cdots \otimes N}^{n}$. Thus the author hopes that any confusion does not occur. The morphism $N^{\otimes n}$ preserves the subspaces $\operatorname{Sym}^{h}(V)$ and $\bigwedge^{h}(V)$. Thus it induces the endomorphisms of $\operatorname{Sym}^{h}(V)$ and $\bigwedge^{h}(V)$. We denote them by $N^{\operatorname{sym} h}$ and $N^{\wedge h}$ respectively.

\subsubsection{The weight filtration of nilpotent maps}

Let $V$ be a finite dimensional vector space over $k$ and $N$ be a nilpotent map of $V$. Recall that $N$ induces the weight filtration $W(N)$ of $V$, which is characterized by the following properties:

- $N \cdot W_{l}(N) \subset W_{l-2}(N)$.

- The induced morphism $N^{k}: G r_{k}^{W(N)} \longrightarrow G r_{-k}^{W(N)}$ is isomorphic for any $k \geq 0$.

We have the following obvious lemma.

Lemma 2.3. Let $\boldsymbol{v}$ be a base of $V$ compatible with the filtration $W(N)$. Then we have the following equality:

$$
\sum_{i} \operatorname{deg}^{W(N)}\left(v_{i}\right)=0 .
$$

For any $l \geq 0$, we put $P_{l} G r_{l}^{W(N)}:=\operatorname{Ker}\left(N^{l+1}: G r_{l}^{W(N)} \longrightarrow\right.$ $\left.G r_{-l-2}^{W(N)}\right)$. When $l-a=2 m \geq 0$ for some nonnegative integer $m$, we put $P_{l} G r_{a}^{W(N)}:=\operatorname{Im}\left(N_{1}^{m}: P_{l} G r_{l}^{W(N)} \longrightarrow G r_{a}\right)$. Then we obtain the decomposition $G r_{a}:=\bigoplus_{0 \leq h} P_{|a|+2 h} G r_{a}$, which is called the primitive decomposition.

\subsubsection{Splittings of the weight filtrations}

Let $s l_{2}$ be a Lie subalgebra of the $(2 \times 2)$-matrix algebra $M(2)$ with the following base:

$$
N_{0}=\left(\begin{array}{ll}
0 & 1 \\
0 & 0
\end{array}\right), \quad N_{1}=\left(\begin{array}{ll}
0 & 0 \\
1 & 0
\end{array}\right), \quad C=\left(\begin{array}{cc}
1 & 0 \\
0 & -1
\end{array}\right) .
$$


Assume that we have a homomorphism of Lie algebras $\eta: s l_{2} \longrightarrow$ $\operatorname{End}(V)$. Then we obtain the weight filtration $W\left(\eta\left(N_{1}\right)\right)$, and the decomposition of $V$ into the eigenspaces of $\eta(C)$ :

$$
V=\bigoplus_{\alpha} V_{\alpha}
$$

Here $\alpha$ runs through the eigenvalues of $\eta(C)$. Then we have the following:

$$
W_{l}\left(\eta\left(N_{1}\right)\right)=\bigoplus_{h \leq l} V_{h}
$$

For any nonnegative integer $n$, we have the naturally induced representation $\eta^{\otimes n}: s l_{2} \longrightarrow \operatorname{End}\left(V^{\otimes n}\right)$. For an $n$-tuple $\boldsymbol{\alpha}=\left(\alpha_{1}, \ldots, \alpha_{n}\right)$ of the eigenvalues of $\eta(C)$, we put $V_{\boldsymbol{\alpha}}:=V_{\alpha_{1}} \otimes \cdots \otimes V_{\alpha_{n}}$. We have the decomposition of $V^{\otimes n}$ as follows:

$$
V^{\otimes n}=\bigoplus_{\alpha} V_{\boldsymbol{\alpha}}
$$

Here $\boldsymbol{\alpha}$ runs through the set of $n$-tuples of the eigenvalues of $\eta(C)$. It is clear that $V_{\boldsymbol{\alpha}}$ is contained in the eigenspace of $\eta^{\otimes n}(C)$ with the eigenvalue $\rho(\boldsymbol{\alpha})=\sum_{i=1}^{n} \alpha_{i}$. Thus we have the eigen decomposition of $V^{\otimes n}$

$$
V^{\otimes n}=\bigoplus_{\alpha}\left(\bigoplus_{\substack{\boldsymbol{\alpha} \\ \rho(\boldsymbol{\alpha})=\alpha}} V_{\boldsymbol{\alpha}}\right)
$$

Here $\alpha$ runs through the eigenvalues of $\eta^{\otimes n}(C)$. Then we obtain the following:

$$
\begin{aligned}
W_{l}\left(\eta\left(N_{1}\right)^{\otimes n}\right) & =\bigoplus_{\substack{\boldsymbol{\alpha} \\
\rho(\boldsymbol{\alpha}) \leq l}} V_{\boldsymbol{\alpha}} \\
& =\left\{\sum x_{1} \otimes \cdots \otimes x_{n} \mid \sum \operatorname{deg}^{W\left(\eta\left(N_{1}\right)\right)}\left(x_{i}\right) \leq l\right\} .
\end{aligned}
$$

Consider the case of the symmetric product and the exterior product. We put as follows for a set $I$ :

$$
\mathcal{S}(I, n):=\left\{f: I \longrightarrow \mathbb{Z}_{\geq 0} \mid \sum_{i \in I} f(i)=n\right\} .
$$


For $f \in \mathcal{S}(I, n)$, we put $\rho(f):=\sum_{i \in I} i \cdot f(i)$.

Let $I$ be the set of the eigenvalues of $\eta(C)$. Then we have the following decomposition:

$$
\operatorname{Sym}^{n}(V)=\bigoplus_{f \in \mathcal{S}(I, n)} \bigotimes_{\alpha \in I} \operatorname{Sym}^{f(\alpha)} V_{\alpha}, \quad \bigwedge^{n}(V)=\bigoplus_{f \in \mathcal{S}(I, n)} \bigotimes_{\alpha \in I}^{f(\alpha)} \bigwedge^{f} V_{\alpha} .
$$

By considering the eigenvalues of $\eta(C)^{\operatorname{sym} n}$ and $\eta(C)^{\wedge n}$, we obtain the following:

$$
\begin{aligned}
W_{l}\left(\eta\left(N_{1}\right)^{\operatorname{sym} n}\right) & =\bigoplus_{\substack{f \in \mathcal{S}(I, n), \rho(f) \leq l}} \bigotimes_{\alpha \in I} \operatorname{Sym}^{f(\alpha)} V_{\alpha} \\
& =\left\{\sum x_{1} \cdots x_{n} \mid \sum \operatorname{deg}^{W\left(\eta\left(N_{1}\right)\right)}\left(x_{i}\right) \leq l\right\} \\
W_{l}\left(\eta\left(N_{1}\right)^{\wedge n}\right) & =\bigoplus_{\substack{f \in \mathcal{S}(I, n), \alpha \in I \\
\rho(f) \leq l}} \bigwedge_{\substack{f(\alpha) \\
V_{\alpha}}} \\
& =\left\{\sum x_{1} \wedge \cdots \wedge x_{n} \mid \sum \operatorname{deg}^{W\left(\eta\left(N_{1}\right)\right)}\left(x_{i}\right) \leq l\right\} .
\end{aligned}
$$

Let $N$ be a nilpotent map on a finite dimensional vector space $V$. We can pick a representation $\eta: s l_{2} \longrightarrow \operatorname{End}(V)$ such that $\eta\left(N_{1}\right)=N$. Thus we obtain the following:

$$
\begin{aligned}
& W_{l}\left(N^{\otimes n}\right)=\left\{\sum x_{1} \otimes \cdots \otimes x_{n} \mid \sum \operatorname{deg}^{W(N)} x_{i} \leq l\right\}, \\
& W_{l}\left(N^{\operatorname{sym} n}\right)=\left\{\sum x_{1} \cdots x_{n} \mid \sum \operatorname{deg}^{W(N)} x_{i} \leq l\right\}, \\
& W_{l}\left(N^{\wedge n}\right)=\left\{\sum x_{1} \wedge \cdots \wedge x_{n} \mid \sum \operatorname{deg}^{W(N)} x_{i} \leq l\right\} .
\end{aligned}
$$

Assume that we have a splitting of the weight filtration $W(N)$, i.e., we have a decomposition $V=\bigoplus_{h} U_{h}$ such that $W(N)_{l}=\bigoplus_{h \leq l} U_{h}$. Then we have the decomposition of the products $V^{\otimes n}, \operatorname{Sym}^{n}(V)$ and $\bigwedge^{n} V$ by the same formula as those (2) and (4), although the meaning is slightly different. They give the splitting of the filtrations $W\left(N^{\otimes n}\right)$, $W\left(N^{\operatorname{sym} n}\right)$ and $W\left(N^{\wedge n}\right)$ by the same formula as those (3) and (5). 


\subsubsection{Compatibility of a commuting tuple of nilpotent maps}

Let $V$ be a finite dimensional vector space with a decomposition $V=$ $\bigoplus V_{i}$. Let $N$ be a nilpotent endomorphism of $V$. Then it induces the weight filtration, which we denote by $W(N)$. Recall that $\underline{n}$ denote the set $\{1, \ldots, n\}$.

Definition 2.5. Let $N_{1}, \ldots, N_{n}$ be a tuple of nilpotent maps of $V$. It is called a commuting tuple, if $N_{i}$ and $N_{j}$ are commutative for any $i, j \in \underline{n}$.

Definition 2.6. Let $\left(N_{1}, \ldots, N_{n}\right)$ be a commuting tuple of nilpotent maps. We say that the constantness of the induced filtration on the positive cones holds, if the following holds:

For any subset $I \subset \underline{n}$, the filtration $W\left(\sum_{i \in I} a_{i} N_{i}\right)$ is independent of $\left(a_{i} \mid i \in I\right) \in \mathbf{R}_{>0}^{I}$.

When the constantness of the filtrations on the positive cone holds, we denote the filtration $W\left(\sum_{i \in I} a_{i} N_{i}\right)\left(a_{i}>0\right)$ by $W(I)$.

Assume that the constantness of the filtrations on the positive cones holds. We put $N(\underline{j})=\sum_{i<j} N_{i}$. Let $W(\underline{j})$ denote the weight filtration of $N(\underline{j})$. We denote the graded vector space associated with $W(\underline{1})$ by $G r^{(1)}$. We have the projection $\pi_{h_{1}}: W(\underline{1})_{h_{1}} \longrightarrow G r_{h_{1}}^{(1)}$. Let $\boldsymbol{h}=$ $\left(h_{1}, \ldots, h_{n}\right)$ denote an $n$-tuple of integers. Then we have the following morphism:

$$
\pi_{h}: W(\underline{1})_{h_{1}} \cap W(\underline{2})_{h_{2}} \cap \cdots \cap W(\underline{n})_{h_{n}} \longrightarrow G r_{h_{1}}^{(1)}
$$

On the other hand, $N(\underline{j})$ induces the morphism $N^{(1)}(\underline{j})$ on $G r^{(1)}$. Let $W\left(N^{(1)}(\underline{j})\right)$ denote the weight filtration of $N^{(1)}(\underline{j})$. Then we have the subspace $G r_{h_{1}}^{(1)} \cap \bigcap_{j=2}^{n} W\left(N^{(1)}(\underline{j})\right)_{h_{j}-h_{1}}$ of $G r_{h_{1}}^{(1)}$.

Definition 2.7. Let $\left(N_{1}, N_{2}, \ldots, N_{l}\right)$ be a commuting tuple of nilpotent maps. It is called sequentially compatible, if the following holds inductively:

- The constantness of the filtrations on the positive cones holds.

- We have the induced tuple $\left(N_{2}^{(1)}, \ldots, N_{l}^{(1)}\right)$ of the commuting nilpotent maps on $G r^{W\left(N_{1}\right)}$. It is sequentially compatible. 
- For any $\boldsymbol{h}=\left(h_{1}, \ldots, h_{n}\right)$, we have

$$
\operatorname{Im}\left(\pi_{\boldsymbol{h}}\right)=G r_{h_{1}}^{(1)} \cap \bigcap_{j=2}^{n} W\left(N^{(1)}(\underline{j})\right)_{h_{j}-h_{1}} .
$$

Remark 2.2. The third condition in Definition 2.7 can be reworded as follows:

- Let $W^{(1)}(j)$ denote the filtration of $G r^{(1)}$ induced by $W(j)$. Then we have the following:

$$
W^{(1)}(\underline{j})_{l+a} \cap G r_{a}^{(1)}=W\left(N^{(1)}(\underline{j})\right)_{l} \cap G r_{a}^{(1)} .
$$

- We have $\operatorname{Im}\left(\pi_{\boldsymbol{h}}\right)=G r_{h_{1}}^{(1)} \cap \bigcap_{j=2}^{n} W^{(1)}(\underline{j})_{h_{j}}$ for any $\boldsymbol{h}=\left(h_{1}, \ldots, h_{n}\right)$ $\in \mathbb{Z}^{n}$.

Remark 2.3. On $G r^{(1)}$, we have $N^{(1)}(\underline{j})=\sum_{i \leq j} N_{i}^{(1)}=\sum_{i=2}^{j} N_{i}^{(1)}$.

Lemma 2.4. Assume that $\left(N_{1}, \ldots, N_{n}\right)$ is compatible. Then $(W(\underline{1})$, $\ldots, W(\underline{n}))$ is a compatible sequence of filtrations.

Proof. We only have to note Remark 2.2.

q.e.d.

Definition 2.8. Assume that $\left(N_{1}, \ldots, N_{n}\right)$ is sequentially compatible. A base $\boldsymbol{v}$ is called compatible with the sequence $\left(N_{1}, \ldots, N_{n}\right)$, if it is compatible with the sequence $(W(\underline{1}), \ldots, W(\underline{n}))$.

For any $n$-tuple $\boldsymbol{h}=\left(h_{1}, \ldots, h_{n}\right)$, we have the following morphism:

$$
P \pi_{\boldsymbol{h}}:\left[\operatorname{Ker}\left(N(\underline{1})^{h_{1}+1}\right) \cap \bigcap_{j=2}^{n} W(\underline{j})_{h_{j}}\right] \longrightarrow P_{h_{1}} G r_{h_{1}}^{(1)} .
$$

Lemma 2.5 When $\left(N_{1}, \ldots, N_{n}\right)$ is a sequentially compatible, we have the following implication:

$$
\operatorname{Im}\left(P \pi_{\boldsymbol{h}}\right) \subset\left[P_{h_{1}} G r_{h_{1}}^{(1)} \cap \bigcap_{j=2}^{n} W^{(1)}(\underline{j})_{h_{j}}\right] .
$$

Proof. We have the following implication:

$\operatorname{Im}\left(P \pi_{\boldsymbol{h}}\right) \subset\left[P_{h_{1}} G r_{h_{1}}^{(1)} \cap \pi_{\boldsymbol{h}}\left(\bigcap_{j=1}^{n} W(\underline{j})_{h_{j}}\right)\right] \subset\left[P_{h_{1}} G r_{h_{1}}^{(1)} \cap \bigcap_{j=2}^{n} W^{(1)}(\underline{j})_{h_{j}}\right]$.

Thus we are done.

q.e.d. 
Definition 2.9. A tuple $\left(N_{1}, \ldots, N_{n}\right)$ is called strongly sequentially compatible if the following holds:

- $\left(N_{1}, \ldots, N_{n}\right)$ is sequentially compatible.

- For any tuple $\boldsymbol{h}=\left(h_{1}, \ldots, h_{n}\right)$, we have the following:

$$
\operatorname{Im}\left(P \pi_{\boldsymbol{h}}\right)=\left[P_{h_{1}} G r_{h_{1}}^{(1)} \cap \bigcap_{j=2}^{n} W^{(1)}(\underline{j})_{h_{j}}\right] .
$$

Definition 2.10. A commuting tuple $\left(N_{1}, \ldots, N_{n}\right)$ of nilpotent maps are called of Hodge type, if the following holds:

- For any permutation $\sigma$ of $\underline{n}$, the tuple $\left(N_{\sigma(1)}, \ldots, N_{\sigma(n)}\right)$ is strongly sequentially compatible.

\subsubsection{Sequential compatibility in the level $h$}

Definition 2.11. Let $\left(N_{1}, N_{2}, \ldots, N_{n}\right)$ be a commuting tuple of nilpotent maps. It is called sequentially compatible in the level $h$, if the following holds:

1. The constantness of the filtrations on the positive cones holds.

2. The induced tuple $\left(N_{2}^{(1)}, \ldots, N_{n}^{(1)}\right)$ on $G r^{W\left(N_{1}\right)}$ is sequentially compatible.

3. For any $\boldsymbol{h}=\left(h_{1}, \ldots, h_{n}\right)$ such that $h_{1} \leq h$, we have $\operatorname{Im}\left(\pi_{\boldsymbol{h}}\right)=$ $G r_{h_{1}}^{(1)} \cap \bigcap_{j=2}^{n} W\left(N^{(1)}(\underline{j})\right)_{h_{j}-h_{1}}$.

In particular, when $h$ is the bottom number of the filtration $W(\underline{1})$, we say that $\left(N_{1}, \ldots, N_{n}\right)$ are sequentially compatible in the bottom part.

Lemma 2.6. Let $\left(N_{1}, \ldots, N_{n}\right)$ be a commuting tuple of nilpotent maps satisfying Conditions 1 and 2 in Definition 2.11. When we check whether $\left(N_{1}, \ldots, N_{n}\right)$ is sequentially compatible in the bottom part, we only have to check the following instead of Condition 3 in Definition 2.11.

4. For any $h$, we have $W(\underline{1})_{b} \cap W(\underline{j})_{h}=G r_{b}^{(1)} \cap W\left(N^{(1)}(\underline{j})\right)_{h-b}$, where $b$ denotes the bottom number of $W(\underline{1})$. 
Proof. It is clear that Condition 3 implies Condition 4. In the bottom part, we have the equality:

$$
W(\underline{1})_{b} \cap \bigcap_{j=2}^{n} W(\underline{j})_{h_{j}}=\bigcap_{j=2}^{n}\left(W(\underline{1})_{b} \cap W(\underline{j})_{h_{j}}\right) .
$$

Thus Condition 4 implies Condition 3.

q.e.d.

Definition 2.12. Let $\left(N_{1}, \ldots, N_{n}\right)$ be a commuting tuple of nilpotent maps. We say that it is universally sequentially compatible in the bottom part, if $\left(N_{1}^{\wedge m}, \ldots, N_{n}^{\wedge m}\right)$ is sequentially compatible in the bottom part for any nonnegative integer $m$.

\subsubsection{Splitting of the sequentially compatible nilpotent maps}

Lemma 2.7. Let $\left(N_{1}, \ldots, N_{n}\right)$ be a commuting tuple, which is sequentially compatible in the level $h$. Then there exists the decomposition of $W(\underline{1})_{h}$ :

$$
W(\underline{1})_{h}=\bigoplus_{k \in \mathbb{Z}^{n}} U_{k}
$$

The decomposition satisfies the following for any $\boldsymbol{h}=\left(h_{1}, \ldots, h_{n}\right)$ such that $h_{1} \leq h$ :

$$
\bigcap_{j=1}^{n} W(\underline{j})_{h_{j}}=\bigoplus_{\boldsymbol{k} \in \mathcal{U}(\boldsymbol{h})} U_{\boldsymbol{k}}, \quad \mathcal{U}(\boldsymbol{h}):=\left\{\boldsymbol{k} \in \mathbb{Z}^{n} \mid \rho_{j}(\boldsymbol{k}) \leq h_{j} j=1, \ldots, n\right\} .
$$

Here we put $\rho_{j}(\boldsymbol{k}):=\sum_{i \leq j} k_{i}$ for $\boldsymbol{k}=\left(k_{1}, \ldots, k_{n}\right)$.

Proof. We have the filtrations $W(\underline{1})_{h} \cap W(j)$ of $W(\underline{1})_{h}$ for any $j$. The sequence $\left(W(\underline{1})_{h} \cap W(\underline{1}), W(\underline{1})_{h} \cap W(\underline{2}), \ldots, W(\underline{1})_{h} \cap W(\underline{n})\right)$ is compatible. Thus we obtain the compatible decomposition:

$$
W(\underline{1})_{h}=\bigoplus_{k \in \mathbb{Z}^{n}} \bar{U}_{k}
$$

For any $\boldsymbol{h} \in \mathbb{Z}^{n}$, we put $\mu(\boldsymbol{h}):=\left(\rho_{1}(\boldsymbol{h}), \rho_{2}(\boldsymbol{h}), \ldots, \rho_{n}(\boldsymbol{h})\right)$. Then we put as follows:

$$
U_{h}=\bar{U}_{\mu(h)}
$$


Then the decomposition $W(\underline{1})_{h}=\bigoplus_{\boldsymbol{h} \in \mathbb{Z}} U_{\boldsymbol{h}}$ has the desired property.

q.e.d.

Let $\left(N_{1}, \ldots, N_{n}\right)$ be a sequentially compatible in the level $h$. We put as follows:

$$
R=\operatorname{dim} W(\underline{1})_{h}, \quad b=\sum_{a \leq h} a \cdot \operatorname{dim} G r_{a}^{(1)} .
$$

We put $\mathcal{V}:=\bigwedge^{R} V$ and $\mathcal{N}_{i}:=N_{i}^{\wedge R}$, and $\mathcal{N}(\underline{j})=\sum_{i \leq j} \mathcal{N}_{i}$.

Lemma 2.8. Consider the weight filtration $\mathcal{W}(\underline{j}):=\mathcal{W}(\mathcal{N}(\underline{j}))$.

- The bottom number of the filtration $\mathcal{W}(\underline{1})$ is $b$.

- We have the natural isomorphism $\mathcal{W}(\underline{1})_{b} \simeq \operatorname{det}\left(W(\underline{1})_{h}\right)$.

- Let e be a nonzero element of $\mathcal{W}(\underline{1})_{b}$. Then we have $\operatorname{deg}^{\mathcal{W}(\underline{m})}(e)=$ $b$ for any $m$.

Proof. The first two claims are clear, and we do not need the sequentially compatibility in the level $h$. (See Subsubsection 2.2.3.) The sequence $(W(\underline{1}), W(\underline{m}))$ is compatible. Take a frame $\boldsymbol{v}$ which is compatible with the sequence $(W(\underline{1}), W(\underline{m}))$. Then we have the following equality:

$$
\operatorname{deg}^{W(\underline{m})}\left(v_{i}\right)=\operatorname{deg}^{W^{(1)}}(\underline{m})\left(v_{i}\right)=\operatorname{deg} W(\underline{1})\left(v_{i}\right)+\operatorname{deg} W^{\left(N^{(1)}(\underline{m})\right)}\left(v_{i}^{(1)}\right) .
$$

We also have the following:

$$
\sum_{\operatorname{deg} W(\underline{1})\left(v_{i}\right)=a} \operatorname{deg}^{W\left(N^{(1)}(\underline{m})\right)}\left(v_{i}^{(1)}\right)=0 .
$$

Then we obtain the equality $\sum_{i} \operatorname{deg}^{W(\underline{m})}\left(v_{i}\right)=\sum_{a \leq h} a \cdot \operatorname{dim} G r_{a}^{(1)}=b$. q.e.d.

\subsubsection{Splitting of strongly sequentially compatible nilpotent maps}

Let $q_{1}: \mathbb{Z}^{n} \longrightarrow \mathbb{Z}$ denote the projection onto the first component. 
Lemma 2.9. Let $\left(N_{1}, \ldots, N_{n}\right)$ be commuting tuple of nilpotent maps, which is strongly sequentially compatible. Then we have the decomposition:

$$
V=\bigoplus_{k \geq 0} \bigoplus_{k \in \mathbb{Z}^{n}} P_{k} U_{k}
$$

It satisfies the following:

1. It gives a splitting of the filtrations $W(\underline{j})$, that is we have the following:

$$
\bigcap_{j=1}^{n} W(\underline{j})_{h_{j}}=\bigoplus_{k \geq 0} \bigoplus_{\boldsymbol{k} \in \mathcal{U}(\boldsymbol{h})} P_{k} U_{\boldsymbol{k}} .
$$

2. $P_{k} U_{\boldsymbol{k}}=0$ unless $\left|q_{1}(\boldsymbol{k})\right| \leq k$ and $k-q_{1}(\boldsymbol{k})$ is even.

3. When $-k<q_{1}(\boldsymbol{k}) \leq k$, we have $N_{1}\left(P_{k} U_{\boldsymbol{k}}\right)=P_{k} U_{\boldsymbol{k}-2 \boldsymbol{\delta}_{1}}$. Here we put $\boldsymbol{k}-2 \boldsymbol{\delta}_{1}=\left(k_{1}-2, k_{2}, \ldots, k_{n}\right)$ for $\boldsymbol{k}=\left(k_{1}, \ldots, k_{n}\right)$.

4. When $q_{1}(\boldsymbol{k})=-k, N_{1}\left(P_{k} U_{\boldsymbol{k}}\right)=0$.

Proof. We have the sequentially compatible tuple $\left(N_{2}^{(1)}, \ldots, N_{n}^{(1)}\right)$ on $P_{h} G r_{h}^{(1)}$. Thus we have the decomposition of $P_{h} G r_{h}^{(1)}$ :

$$
\begin{aligned}
P_{h} G r_{h}^{(1)} & =\bigoplus_{\boldsymbol{k} \in \mathbb{Z}^{n-1}} P_{h} U_{h, \boldsymbol{k}}, \quad P_{h} G r_{h}^{(1)} \cap \bigcap_{j=1}^{n-1} W\left(N^{(1)}(\underline{j+1})\right)_{h_{j}^{\prime}} \\
& =\bigoplus_{\boldsymbol{k} \in \mathcal{U}\left(\boldsymbol{h}^{\prime}\right)} P_{h} U_{h, \boldsymbol{k}} .
\end{aligned}
$$

For a tuple $\boldsymbol{h}=\left(h_{1}, \ldots, h_{n}\right)$, we put $\chi(\boldsymbol{h}):=\left(h_{1}, h_{2}-h_{1}, h_{3}-h_{2}, \ldots\right.$, $\left.h_{n}-h_{n-1}\right)$, and $\chi^{\prime}(\boldsymbol{h})=\left(h_{2}-h_{1}, h_{3}-h_{2}, \ldots, h_{n}-h_{n-1}\right)$. Note that $\chi(\boldsymbol{h}) \in \mathcal{U}(\boldsymbol{h})$. When we put $\boldsymbol{h}^{\prime}=\left(h_{2}-h_{1}, \ldots, h_{n}-h_{1}\right) \in \mathbb{Z}^{n-1}$, we have $\chi^{\prime}(\boldsymbol{h}) \in \mathcal{U}\left(\boldsymbol{h}^{\prime}\right)$.

Since $\left(N_{1}, \ldots, N_{n}\right)$ is strongly sequentially compatible, the space $P_{h_{1}} U_{h_{1}, \chi^{\prime}(\boldsymbol{h})}$ is contained in the image of $P \pi_{\boldsymbol{h}}$ :

$$
\begin{aligned}
& P \pi_{\boldsymbol{h}}\left[\operatorname{Ker}\left(N(1)^{h_{1}+1}\right) \cap \bigcap_{j=2}^{n} W(\underline{j})_{h_{j}}\right] \\
& =\left[P_{h_{1}} G r_{h_{1}}^{(1)} \cap \bigcap_{j=2}^{n} W\left(N^{(1)}(\underline{j})\right)_{h_{j}-h_{1}}\right] \supset P_{h_{1}} U_{h_{1}, \chi^{\prime}(\boldsymbol{h})} .
\end{aligned}
$$


Thus we can take a subspace $P_{h_{1}} U_{\chi(\boldsymbol{h})}$ of $\operatorname{Ker}\left(N(1)^{h_{1}+1}\right) \cap \bigcap_{j=2}^{n} W(\underline{j})_{h_{j}}$ such that $P_{h_{1}} U_{\chi(\boldsymbol{h})}$ is isomorphic to $P_{h_{1}} U_{h_{1}, \chi^{\prime}(\boldsymbol{h})}$ via the morphism $\bar{P} \pi_{\boldsymbol{h}}$.

For an integer $m$ such that $0 \leq m \leq h_{1}$, we put as follows:

$$
P_{h_{1}} U_{\boldsymbol{h}-2 m \boldsymbol{\delta}_{1}}:=N_{1}^{m}\left(P_{h_{1}} U_{\boldsymbol{h}}\right) .
$$

Here we put $\boldsymbol{h}-2 m \cdot \boldsymbol{\delta}_{1}=\left(h_{1}-2 m, h_{2}, \ldots, h_{n}\right)$ for $\boldsymbol{h}=\left(h_{1}, h_{2}, \ldots, h_{n}\right)$. By our choice, we have $N_{1}^{h_{1}+1}\left(P_{h_{1}} U_{\boldsymbol{h}}\right)=0$. Then we obtain the desired decomposition.

q.e.d.

For any tuple $\boldsymbol{h} \in \mathbb{Z}^{n}$ and $k \geq 0$, we have the number $d(k, \boldsymbol{h}):=$ $\operatorname{dim} P_{k} U_{\boldsymbol{h}}$. Clearly we have $d(k, \boldsymbol{h})=d(k-2, \boldsymbol{h})$ if $-k<q_{1}(\boldsymbol{h}) \leq k$.

Corollary 2.1. Assume that $\left(N_{1}, \ldots, N_{n}\right)$ is strongly sequentially compatible. Then we can take a base $\boldsymbol{v}$ of $V$ satisfying the following:

1. $\boldsymbol{v}=\left(v_{k, \boldsymbol{h}, \eta} \mid k \geq 0, \boldsymbol{h} \in \mathbb{Z}^{n}, \eta=1, \ldots, d(k, \boldsymbol{h})\right)$.

2. We have $N_{1}\left(v_{k, \boldsymbol{h}, \eta}\right)=v_{k, \boldsymbol{h}-2 \boldsymbol{\delta}_{1}, \eta}$ when $-k<q_{1}(\boldsymbol{h}) \leq k$.

3. $N_{1}\left(v_{k, \boldsymbol{h}, \eta}\right)=0$ if $q_{1}(\boldsymbol{h})=-k$.

4. $\operatorname{deg}^{W(\underline{j})}\left(v_{k, \boldsymbol{h}, \eta}\right)=\rho_{j}(\boldsymbol{h})$.

5. $\boldsymbol{v}$ is compatible with the sequence $(W(\underline{1}), \ldots, W(\underline{n}))$.

Proof. We take a base $\left(v_{h_{1}, \boldsymbol{h}, \eta} \mid \eta=1, \ldots, d\left(h_{1}, \boldsymbol{h}\right)\right)$ of $P_{h_{1}} U_{\boldsymbol{h}}$ in the case $\boldsymbol{h}=\left(h_{1}, \ldots, h_{n}\right)$. We put $v_{h_{1}, \boldsymbol{h}-2 m \boldsymbol{\delta}_{1}, \eta}:=N_{1}^{m}\left(v_{h_{1}, \boldsymbol{h}, \eta}\right)$. Then we obtain the frame desired.

q.e.d.

Definition 2.13. A frame $\boldsymbol{v}$ satisfying the condition in Corollary 2.1 is called strongly compatible with $(N(\underline{1}), N(\underline{2}), \ldots, N(\underline{n}))$.

\subsubsection{A reduction of the sequential compatibility}

Proposition 2.1. Let $\left(N_{1}, \ldots, N_{n}\right)$ be a commuting tuple. Assume the following:

- $\left(N_{1}, N_{2}, \ldots, N_{n}\right)$ is universally sequentially compatible in the bottom parts.

- $(W(\underline{1}), \ldots, W(\underline{n}))$ is compatible.

Then $\left(N_{1}, N_{2}, \ldots, N_{n}\right)$ is sequentially compatible. 
Proof. We only have to show that $\left(N_{1}, \ldots, N_{n}\right)$ is sequentially compatible in the level $h$ for any $h$. We use an induction on $h$. We assume that we have already known that $\left(N_{1}, \ldots, N_{n}\right)$ is sequentially compatible in the level $h-1$.

We put as follows:

$$
R_{1}:=\operatorname{dim} W(\underline{1})_{h-1}+1, \quad b_{1}:=\sum_{a \leq h-1} a \cdot \operatorname{dim} G r_{a}^{(1)}+h .
$$

We put $\mathcal{V}_{1}:=\bigwedge^{R_{1}} V, \mathcal{N}_{i}:=N_{i}^{\wedge} R_{1}$ and $\mathcal{N}(\underline{j})=\sum_{i \leq j} \mathcal{N}_{i}$. We obtain the weight filtration $\mathcal{W}(j)$ of $\mathcal{N}(j)$. We denote the associated graded vector space of $\mathcal{W}(\underline{1})$ by $\mathcal{G} r^{(1)}$. Then the bottom number of the filtration $\mathcal{W}(\underline{1})$ is $b_{1}$ above. The tuple $\left(\mathcal{N}_{1}, \ldots, \mathcal{N}_{n}\right)$ is sequentially compatible in the bottom part. Thus we have the following equality for any $\left(h_{2}, \ldots, h_{n}\right)$ :

$$
\mathcal{W}(\underline{1})_{b_{1}} \cap \bigcap_{j=2}^{n} \mathcal{W}(\underline{j})_{h_{j}}=\mathcal{G} r_{b_{1}}^{(1)} \cap \bigcap_{j=2}^{n} \mathcal{W}\left(\mathcal{N}^{(1)}(\underline{j})\right)_{h_{j}-b_{1}} .
$$

Due to our choice of $R_{1}$, we have the natural isomorphism: $\mathcal{G} r_{b_{1}}^{(1)}=$ $\mathcal{W}(\underline{1})_{b_{1}} \simeq \operatorname{det}\left(W(\underline{1})_{h-1}\right) \otimes G r_{h}^{(1)}$. Under the isomorphism, the morphisms $\mathcal{N}_{i}^{(1)}$ and $N_{i}^{(1)}$ also correspond. Thus we obtain the following equality for any $h$ and $\left(h_{2}, \ldots, h_{n}\right)$ under the isomorphism:

$$
\begin{aligned}
& \mathcal{G} r_{b_{1}}^{(1)} \cap \bigcap_{j=2}^{n} \mathcal{W}\left(\mathcal{N}^{(1)}(\underline{j})\right)_{h_{j}-h} \\
& =\operatorname{det}\left(W(\underline{1})_{h-1}\right) \otimes\left[G r_{h}^{(1)} \cap \bigcap_{j=2}^{n} W\left(N^{(1)}(\underline{j})\right)_{h_{j}-h}\right] .
\end{aligned}
$$

Thus we only have to check the following coincidence. (Here we put $h_{1}=h$ for simplicity of notation):

$$
\bigcap_{j=1}^{n} \mathcal{W}(\underline{j})_{h_{j}-h+b_{1}}=\operatorname{det}\left(W(\underline{1})_{h-1}\right) \otimes\left[\operatorname{Im}\left(\pi_{\boldsymbol{h}}: \bigcap_{j=1}^{n} W(\underline{j})_{h_{j}} \longrightarrow G r_{h}^{(1)}\right)\right] .
$$

First we see that $\operatorname{det}\left(W(\underline{1})_{h-1}\right) \otimes \operatorname{Im}\left(\pi_{\boldsymbol{h}}\right)$ is contained in $\bigcap_{j=1}^{n} \mathcal{W}(\underline{j})_{h_{j}-h_{1}+b}$. Take a nonzero element $e$ of $\operatorname{det}\left(W(\underline{1})_{h-1}\right)$. We know that the degree of $e$ in $\bigwedge^{R} V$ with respect to the filtration 
$W\left(N(j)^{\wedge R}\right)$ is $b=b_{1}-h_{1}$ for any $j$. Here $R$ and $b$ are given as follows: (See $(\overline{7})$. We use $h-1$ instead of $h$.)

$$
R:=\operatorname{dim} W(\underline{1})_{h-1}, \quad b:=\sum_{a \leq h-1} a \cdot \operatorname{dim} G r_{a}^{(1)}=b_{1}-h_{1} .
$$

Thus we obtain the following inequality:

$$
\operatorname{deg}^{\mathcal{W}(\underline{j})}(e \wedge y) \leq b_{1}-h_{1}+h_{j} .
$$

It implies that $e \wedge y \in \bigcap_{j=1}^{n} \mathcal{W}(\underline{j})_{b_{1}-h_{1}+h_{j}}$.

Consider the implication

$$
\bigcap_{j=1}^{n} \mathcal{W}(\underline{j})_{b_{1}-h_{1}+h_{j}} \subset \operatorname{det}\left(W(\underline{1})_{h-1}\right) \otimes \operatorname{Im}\left(\pi_{\boldsymbol{h}}\right) .
$$

Any element of $\bigcap_{j=1}^{n} \mathcal{W}(\underline{j})_{b_{1}-h_{1}+h_{j}}$ is described as follows:

$$
e \wedge y, \quad y \in W(\underline{1})_{h_{1}} \text {. }
$$

Consider the splitting $V=\bigoplus_{k \in \mathbb{Z}^{n}} U_{\boldsymbol{k}}$ compatible with the compatible tuple of the filtrations $(W(\underline{1}), \ldots, W(\underline{n}))$. Then we have the following decomposition:

$$
y=\sum_{k \in \mathbb{Z}^{n}} y_{k}, \quad y_{k} \in U_{k} .
$$

Due to the condition $\operatorname{deg}^{\mathcal{W}(\underline{n})}(e \wedge y) \leq h_{n}-h_{1}+b$, we have the following vanishing for any $l>h_{n}$ :

$$
e \wedge \sum_{\substack{k \in \mathbb{Z}^{n} \\ \rho_{n}(\boldsymbol{k})=l}} y_{\boldsymbol{k}}=0 .
$$

It implies that $\sum_{\rho_{n}(\boldsymbol{k})=l} y_{\boldsymbol{k}} \in W(\underline{1})_{h-1}$. We put as follows:

$$
y^{\prime}=y-\sum_{l>h_{n}} \sum_{\substack{\boldsymbol{k} \in \mathbb{Z}^{n} \\ \rho_{n}(\boldsymbol{k})=l}} y_{k} .
$$

Then we know the following:

$$
e \wedge y^{\prime}=e \wedge y, \quad y^{\prime} \in W(\underline{1})_{h} .
$$

Thus we can assume that $y_{\boldsymbol{k}}=0$ if $\rho_{n}(\boldsymbol{k})>h_{n}$, from the beginning.

By an inductive argument, we can assume that $y_{k}=0$ if there exist $1 \leq j \leq n$ such that $\rho_{j}(\boldsymbol{k})>h_{j}$. In that case, $y$ is contained in $\bigcap_{j=1}^{n} W(\underline{j})_{h_{j}}$. It implies the implication desired. Thus we are done.

q.e.d. 


\subsubsection{A lemma of Cattani-Kaplan}

Let $N_{1}, \ldots, N_{n}$ be a commuting tuple of nilpotent maps on $V$. Let $t_{1} \ldots, t_{n}$ be formal variables and we put $N(\boldsymbol{t}):=\sum t_{i} \cdot N_{i}$. Let $K$ denote the rational function field $\mathbf{C}\left(t_{1}, \ldots, t_{n}\right)$ with variables $t_{1}, \ldots, t_{n}$. Then $N(\boldsymbol{t})$ gives a nilpotent map over $V \otimes_{\mathbf{C}} K$. The weight filtration induced by $N(\boldsymbol{t})$ is denoted by $W(\boldsymbol{t})$.

Let $\boldsymbol{a}=\left(a_{1}, \ldots, a_{n}\right)$ be an element of $\mathbf{C}^{n}$. Then we have $N(\boldsymbol{a})=$ $\sum a_{i} \cdot N_{i}$. We denote the weight filtration of $N(\boldsymbol{a})$ by $W(\boldsymbol{a})$.

Definition 2.14. When we have $\operatorname{dim}_{\mathbf{C}}\left(W(\boldsymbol{a})_{l}\right)=\operatorname{dim}_{K}\left(W(\boldsymbol{t})_{l}\right)$ for any $l$, we say that $\boldsymbol{a}$ is general, or that $N(\boldsymbol{a})$ is general.

Since $N_{i}$ are commuting, we always have $N_{i} \cdot W(\boldsymbol{a})_{l} \subset W_{l}(\boldsymbol{a})$.

Lemma 2.10 (Cattani-Kaplan, [5]). When $N(\boldsymbol{a})$ is general, we have $N_{i} \cdot W(\boldsymbol{a})_{l} \subset W(\boldsymbol{a})_{l-1}$.

\subsubsection{A lemma for the conjugacy classes of the nilpotent maps}

We recall some general result on the conjugacy classes of nilpotent maps. We will use the result later without mention. Let $R$ be a discrete valuation ring. Let $K$ and $k$ denote the quotient field and the residue field of $R$ respectively. Let $V$ be a free module over a discrete valuation ring $R$. Let $N$ be a nilpotent maps of $V$ defined over $R$. We put $V_{K}:=V \otimes_{R} K$ and $V_{k}:=V \otimes_{R} k$. We have the induced nilpotent maps $N_{K} \in \operatorname{End}\left(V_{K}\right)$ and $N_{k} \in \operatorname{End}\left(V_{k}\right)$. They induce the weight filtrations $W_{K}$ and $W_{k}$ of $V_{K}$ and $V_{k}$ respectively.

Lemma 2.11. We put $l_{0}:=\min \left\{l \mid \operatorname{dim} W_{K, l} \neq \operatorname{dim} W_{k, l}\right\}$. Then we have the following inequality:

$$
\operatorname{dim}_{K} W_{K, l_{0}}>\operatorname{dim}_{k} W_{k, l_{0}} .
$$

Proof. First observe the following: Let $b(K)$ and $b(k)$ be the bottom numbers of the filtrations $W_{K}$ and $W_{k}$. If $N_{K}^{l}=0$, then $N^{l}=0$ and thus $N_{k}^{l}=0$. It implies that $b(K) \leq b(k)$. If $b(K)=b(k)=b$, then we have the following inequality:

$$
\operatorname{dim} W_{K, b(K)}=\operatorname{dim} \operatorname{Im}\left(N_{K}^{b}\right) \geq \operatorname{dim} \operatorname{Im}\left(N_{k}^{b}\right)=\operatorname{dim} W_{k, b(k)} .
$$

We put $D=\sum_{l<l_{0}} \operatorname{dim} W_{K, l}$. By considering the exterior product $\bigwedge^{D+1} V$, we can reduce the problem to the comparison of the dimension of the bottom parts. Thus we are done.

q.e.d. 


\subsection{Vector bundles and filtrations}

\subsubsection{Words and Notation}

Let $X$ be a complex manifold and $E$ be a $C^{\infty}$-vector bundle over $\mathbf{C}$. The space of $C^{\infty}$-sections are denoted by $C^{\infty}(X, E)$. When $E$ is a holomorphic bundle, the space of holomorphic sections is denoted by $\Gamma(X, E)$. For frame $\boldsymbol{v}$ and $\boldsymbol{w}$, we have the transformation matrices $B$ determined by $\boldsymbol{v}=\boldsymbol{w} \cdot B$.

Let $h$ be a hermitian metric of $E$, and $\boldsymbol{v}$ be a frame of $E$ of rank $r$. Then we obtain the $\mathcal{H}(r)$-valued function $H(h, \boldsymbol{v})$ and $H(h, \boldsymbol{v})^{-1}$.

Definition 2.15. A frame $\boldsymbol{v}$ is called adapted, if $H(h, \boldsymbol{v})$ and $H(h, \boldsymbol{v})^{-1}$ are bounded over $X$.

Let $Y$ be a subset of $X$. The restriction of $E$ to $Y$ is denoted by $E_{\mid Y}$.

Assume that a decomposition of $E_{\mid Y}$ into a direct sum of vector bundles $\bigoplus E_{i}$ is given. The restriction of a $C^{\infty}$-section $f$ of $E$ to $Y$ is denoted by $f_{\mid Y}$. It is called compatible with the decomposition, if there is an $i$ such that $f_{\mid Y}$ is a section of $E_{i}$. A frame $\boldsymbol{v}=\left(v_{1}, \ldots, v_{n}\right)$ of $E$ is compatible with the decomposition, if each $v_{i}$ is compatible with the decomposition.

\subsubsection{Filtrations}

A filtration $W$ of $E_{\mid Y}$ by vector bundle is defined to be a finite increasing sequence of vector subbundles:

$$
W_{a} \subset W_{a+1} \subset \cdots \subset W_{a+h} \subset E_{\mid Y} .
$$

The associated graded vector bundle on $Y$ is denoted by $G r^{W}\left(E_{\mid Y}\right)$.

If $E$ is a holomorphic vector bundle, then a filtration of $E$ by subsheaves is defined to be a similar finite increasing sequence of subsheaves.

When a decomposition of $E_{\mid Y}$ is given, a filtration $W$ of $E_{\mid Y}$ is called compatible with the decomposition if $W_{l}=\bigoplus_{i} E_{i} \cap W_{l}$.

Definition 2.16. Let $W$ be a filtration of $E_{\mid Y}$. A $C^{\infty}$-section $f$ of $E$ is called compatible with the filtration $W$, if the numbers $\operatorname{deg}^{W_{\mid P}}(f(P))$ are independent of $P \in Y$. In that case, we put $\operatorname{deg}^{W}(f):=\operatorname{deg}^{W}(f(P))$ for some $P \in Y$.

Definition 2.17. Let $\boldsymbol{v}=\left(v_{1}, \ldots, v_{n}\right)$ be a $C^{\infty}$-frame of $E$. It is called compatible with the filtration $W$ of $E_{\mid Y}$, if the following conditions are satisfied: 
1. Each $v_{i}$ is compatible with the filtration $W$.

2. For any point $P \in Y$, the frame $\boldsymbol{v}_{\mid P}$ is compatible with the filtration $W_{\mid P}$.

The induced sections $\boldsymbol{v}^{(1)}=\left(v_{1}^{(1)}, \ldots, v_{n}^{(1)}\right)$ on $Y$ gives a frame of $G r^{W}\left(E_{\mid Y}\right)$ compatible with the decomposition.

We put $X=\Delta^{n}, D_{i}=\left\{z_{i}=0\right\}$ and $D=\bigcup_{i=1}^{l} D_{i}$ for $l \leq n$. We put $D_{I}=\bigcup_{i \in I} D_{i}$ for $I \subset \underline{l}$. Let $\sigma$ be an element of the $l$-th symmetric group $\mathfrak{S}_{l}$. Then we obtain the sequence of the subset:

$$
I_{1} \subset I_{2} \subset \cdots \subset I_{l}, \quad I_{j}=\{\sigma(i) \mid i \leq j\} .
$$

Then we obtain the sequence $D_{I_{1}} \supset D_{I_{2}} \supset \cdots \supset D_{I_{l}}$.

Definition 2.18. Let $E$ be a vector bundle over $X$. A sequence $\left(W\left(I_{1}\right), W\left(I_{2}\right), \ldots, W\left(I_{l}\right)\right)$ of the filtration of $E$ is the following data:

1. For any point $Q \in D_{I_{m}}$, the sequence $\left(W\left(I_{1}\right)_{\mid Q}, W\left(I_{2}\right)_{\mid Q}, \ldots\right.$, $\left.W\left(I_{m}\right)_{\mid Q}\right)$ is a compatible sequence of filtrations.

2. $W\left(I_{j}\right)$ is a filtration of $E_{\mid D_{I_{j}}}$ by vector subbundles.

3. Let $\boldsymbol{h}=\left(h_{1}, \ldots, h_{m}\right)$ denote a tuple of integers. We have a vector spaces $\bigcap_{j=1}^{m} W\left(I_{j}\right)_{h_{j} \mid Q}$ for any $Q \in D_{I_{m}}$. Then they form a vector subbundle of $E_{\mid D_{I m}}$. Namely we have a vector subbundle $\bigcap_{j=1}^{m} W\left(I_{j}\right)_{h_{j} \mid D_{I_{m}}}$ of $E_{\mid D_{I_{m}}}$.

Let $Q \in D_{I_{m}}$. Then for any tuple $\boldsymbol{h} \in \mathbb{Z}^{m}$, we have the number $d(\boldsymbol{h})=\operatorname{dim} U_{\boldsymbol{h}}$ for a splitting $E_{Q}=\bigoplus_{\boldsymbol{k} \in \mathbb{Z}} U_{\boldsymbol{k}}$ compatible with the filtrations $\left(W\left(I_{1}\right)_{\mid D_{I_{m}}}, \ldots, W\left(I_{m}\right)_{\mid D_{I_{m}}}\right)$.

Lemma 2.12. For a tuple $\boldsymbol{h} \in \mathbb{Z}^{m}$, the number $d(\boldsymbol{k})$ is independent of a choice of $Q \in D_{I_{m}}$.

Proof. As is easily seen, the number $d(\boldsymbol{k})$ is determined by the following numbers:

$$
\operatorname{dim}\left[\bigcap_{j=1}^{m} W\left(I_{j}\right)_{h_{j} \mid Q}\right], \quad\left(h_{1}, \ldots, h_{m}\right) \in \mathbb{Z}^{m} .
$$

By our assumption, the numbers are independent of a choice of $Q$. q.e.d.

On $D\left(I_{1}\right)$, we obtain the graded vector space $\mathcal{G} r^{(1)}$ associated with the filtration $W\left(I_{1}\right)$. For any point $Q \in D_{\underline{m}}$, we have the induced filtration $W^{(1)}(\underline{m})_{\mid Q}$ of $\mathcal{G} r_{\mid Q}^{(1)}$. 
Lemma 2.13. $\left\{W^{(1)}\left(I_{m}\right)_{\mid Q} \mid Q \in D_{I_{m}}\right\}$ gives a filtration of $E_{\mid D_{\underline{m}}}$ by vector subbundles.

Proof. We only have to check that the dimension of $W^{(1)}\left(I_{m}\right)_{\mid Q}$ is independent of a choice of $Q \in D_{I_{m}}$. It follows from the fact that the numbers $d(\boldsymbol{h})$ are independent of a choice of $Q \in D_{I_{m}}$. $\quad$ q.e.d.

For any $\boldsymbol{l} \in \mathbb{Z}^{m-1}$, we have the vector subspaces

$$
\left\{\bigcap_{j=1}^{m-1} W^{(1)}\left(I_{j+1}\right)_{l_{j} \mid Q} \mid Q \in D_{I_{m}}\right\} .
$$

Lemma 2.14. For each $\boldsymbol{l} \in \mathbb{Z}^{m-1},\left\{\bigcap_{j=1}^{m-1} W^{(1)}\left(I_{j+1}\right)_{l_{j} \mid Q} \mid Q \in\right.$ $\left.D_{I_{m}}\right\}$ forms a vector subbundle over $D_{I_{m}}$.

Proof. Again we only have to see the independence of the dimension of the vector spaces $\bigcap_{j=1}^{m-1} W^{(1)}\left(I_{j+1}\right)_{l_{j} \mid Q}$. It follows from the fact that the numbers $d(\boldsymbol{h})$ are independent of a choice of $Q \in D_{I_{m}}$. $\quad$ q.e.d.

We have the vector bundle $\mathcal{G} r^{(1)}$ on $D_{I_{1}}$, and the filtrations $W^{(1)}\left(I_{j}\right)$ on $D_{I_{j}}$ for $j \geq 1$. We have already seen the following proposition in Lemmas 2.13 and 2.14 .

Proposition 2.2. $\left(W^{(1)}\left(I_{2}\right), \ldots, W^{(1)}\left(I_{l}\right)\right)$ is a compatible sequence of filtrations.

Definition 2.19. Let $f$ be a section of $E$ over $X$. We say that $f$ is compatible with the sequence of the filtrations $\left(W\left(I_{1}\right), W\left(I_{2}\right), \ldots\right.$, $W\left(I_{l}\right)$ ), if the following is satisfied:

- $f$ is compatible with $W\left(I_{j}\right)$ for any $j$.

- Let $P$ be a point of $D_{I_{j}}$. Then $f_{\mid P}$ is compatible with the filtrations $\left(W\left(I_{1}\right)_{\mid P}, \ldots, W\left(I_{j}\right)_{\mid P}\right)$ q.e.d.

Definition 2.20. Let $\boldsymbol{v}$ be a frame of $E$. We say $\boldsymbol{v}$ is compatible with the sequence $\left(W\left(I_{1}\right), \ldots, W\left(I_{l}\right)\right)$ if the following holds:

- $\boldsymbol{v}$ is compatible with the filtration $W\left(I_{j}\right)$ for any $j$.

- Let $P$ be a point of $I_{j}$. Then $\boldsymbol{v}_{\mid P}$ is compatible with the sequence $\left(W\left(I_{1}\right)_{\mid P}, \ldots, W\left(I_{j}\right)_{\mid P}\right)$. 


\subsubsection{The existence of compatible splitting}

Let $\left(W\left(I_{1}\right), \ldots, W\left(I_{l}\right)\right)$ be a compatible sequence of filtrations. For simplicity of notation, we assume that $I_{j}=\underline{j}=\{1, \ldots, j\}$.

Lemma 2.15. For any $1 \leq m \leq l$, there are decompositions of $E_{\mid D_{\underline{m}}}$ :

$$
E_{\mid D_{\underline{m}}}=\bigoplus_{\boldsymbol{h} \in \mathbb{Z}^{m}} \mathcal{K}_{\boldsymbol{h}}
$$

They satisfy the following:

1. For any $\boldsymbol{h} \in \mathbb{Z}^{m}$, we have $\bigcap_{j=1}^{m} W(\underline{j})_{h_{j}}=\bigoplus_{\boldsymbol{k} \in \mathcal{T}(\boldsymbol{h})} \mathcal{K}_{\boldsymbol{k}}$ on $D_{\underline{m}}$. Here $\mathcal{T}(\boldsymbol{h})$ denotes the set of $\boldsymbol{k} \in \overline{\mathbb{Z}}^{m}$ satisfying $q_{j}(\boldsymbol{k}) \leq h_{j} \overline{\text { for }}$ any $1 \leq j \leq m$.

2. We have $\mathcal{K}_{\boldsymbol{h} \mid D_{m+1}}=\bigoplus_{k} \mathcal{K}_{(\boldsymbol{h}, k)}$. Here $(\boldsymbol{h}, k)=\left(h_{1}, \ldots, h_{m}, k\right)$ for $\boldsymbol{h}=\left(h_{1}, \ldots, h_{m}\right)$.

Proof. We use an induction on $l$. We have the vector bundle $\mathcal{G} r_{h}^{(1)}$ on $D_{\underline{1}}$, and the filtrations $\mathcal{G} r_{h}^{(1)} \cap W^{(1)}(\underline{j})$ on $D_{j}$. Since the sequence of the filtration is compatible, we have the compatible splitting:

$$
\mathcal{G} r_{h \mid D_{\underline{m}}}^{(1)}=\bigoplus_{\boldsymbol{k}^{\prime} \in \mathbb{Z}^{m-1}} \mathcal{K}_{h, \boldsymbol{k}^{\prime}}
$$

We construct $\mathcal{K}_{\boldsymbol{h}}$ on $D_{\underline{m}}$ by using an descending induction on $m$. Assume that we have already constructed $\mathcal{K}_{\boldsymbol{h}}$ on $D_{\underline{m+1}}$, and consider the decomposition on $D_{\underline{m}}$.

For a tuple $\boldsymbol{h}=\left(h_{1}, \ldots, h_{m}\right) \in \mathbb{Z}^{m}$, we put $\boldsymbol{h}^{\prime}=\left(h_{2}, \ldots, h_{m}\right)$. Then $\mathcal{K}_{h_{1}, \boldsymbol{h}^{\prime}}$ is contained in the image of the following morphism on $D_{\underline{m}}$, by our assumption:

$$
\pi_{\boldsymbol{h}}: \bigcap_{j=1}^{m} W(\underline{j})_{h_{j}} \longrightarrow \mathcal{G} r_{h_{1}}^{(1)} \cap \bigcap_{j=2}^{m} W^{(1)}(\underline{j})_{h_{j}} \supset \mathcal{K}_{h_{1}, \boldsymbol{h}^{\prime}}
$$

On $D_{m+1}$, we already have $\bigoplus_{k} \mathcal{K}_{(\boldsymbol{h}, k)}$. By extending it, we can take a subbundle $\mathcal{K}_{\boldsymbol{h}}$ of $\bigcap_{j=1}^{m} W(\underline{j})_{h_{j}}$ on $D_{\underline{m}}$, satisfying the following:

- $\mathcal{K}_{\boldsymbol{h}}$ is isomorphic to $\mathcal{K}_{h_{1}, \boldsymbol{h}^{\prime}}$ via the morphism $\pi_{\boldsymbol{h}}$.

- We have $\mathcal{K}_{\boldsymbol{h} \mid D_{\underline{m+1}}}=\bigoplus_{k} \mathcal{K}_{(\boldsymbol{h}, k)}$. 
Thus the induction can proceed.

q.e.d.

Definition 2.21. Such tuple $\left\{\mathcal{K}_{\boldsymbol{h}} \mid \boldsymbol{h} \in \mathbb{Z}^{m}, m=1, \ldots, l\right\}$ is called a compatible splitting of the sequence $(W(\underline{1}), \ldots, W(\underline{l}))$.

Lemma 2.16. Let $(W(\underline{1}), \ldots, W(\underline{l}))$ be a compatible sequence of filtrations of $E$. There exists a frame $\boldsymbol{v}$ of $E$ compatible with the sequence $(W(\underline{1}), \ldots, W(\underline{l}))$.

Proof. We take a compatible splitting $\left\{\mathcal{K}_{\boldsymbol{h}} \mid \boldsymbol{h} \in \mathbb{Z}^{m}, m=1, \ldots, l\right\}$ of the sequence $(W(\underline{1}), \ldots, W(\underline{l}))$. We can take a frame $\boldsymbol{v}$ compatible with the splitting $\left\{\mathcal{K}_{\boldsymbol{h}} \mid \boldsymbol{h} \in \mathbb{Z}^{m}, m=1, \ldots, l\right\}$. Thus we are done.

q.e.d.

\subsection{Commuting tuple for a vector bundle}

\subsubsection{Constantness of the filtrations on the positive cones}

Let $E$ be a holomorphic vector bundle over $\Delta^{n}$. Let $l$ be a natural number such that $l \leq n$. We put $D_{i}:=\left\{\left(z_{1}, \ldots, z_{n}\right) \in \Delta^{n} \mid z_{i}=0\right\}$, and $D_{\underline{m}}=\bigcap_{i=1}^{m} D_{i}$. Let $N_{i}$ be an element of $\Gamma\left(D_{i}, \operatorname{End}(E)_{\mid D_{i}}\right)$ for $i=1, \ldots, l$. For $m \leq l$, we have the nilpotent maps $N_{1}, \ldots, N_{m}$ of $\operatorname{End}\left(E_{\mid D_{\underline{m}}}\right)$ on $D_{\underline{m}}$. Then we put as follows, for any $\boldsymbol{a} \in \mathbf{R}_{\geq 0}^{m}$ :

$$
N(\boldsymbol{a}):=\sum_{j=1}^{m} a_{j} \cdot N_{j \mid D_{\underline{m}}} .
$$

Definition 2.22. We say that the constantness of the the filtrations on the positive cones for $\left(N_{1}, \ldots, N_{l}\right)$ holds, if the following holds:

- For any $m \leq l$, for any $Q \in D_{\underline{m}}$ and for any $I \subset \underline{m}$, the filtration $W(\boldsymbol{a})_{\mid Q}$ is independent of a choice of $\boldsymbol{a} \in \mathbf{R}_{>0}^{I}$.

- $\left\{W(\boldsymbol{a})_{\mid Q} \mid Q \in D_{\underline{m}}\right\}$ forms the vector bundle on $D_{\underline{m}}$.

\subsubsection{Sequential compatibility}

Let $E$ be a holomorphic vector bundle over $\Delta^{n}$. We put $D_{i}:=\left\{\left(z_{1}, \ldots\right.\right.$, $\left.\left.z_{n}\right) \in \Delta^{n} \mid z_{i}=0\right\}$, and $D_{\underline{m}}=\bigcap_{i=1}^{m} D_{i}$. Let $l$ be a number less than $n$. Let $N_{i}$ be an element of $\Gamma\left(D_{i}, \operatorname{End}(E)_{\mid D_{i}}\right)$ for $i=1, \ldots, l$. On $D_{\underline{m}}$, we have the nilpotent maps $N_{1 \mid D_{\underline{m}}}, \ldots, N_{m \mid D_{\underline{m}}}$ of $\operatorname{End}\left(E_{\mid D_{\underline{m}}}\right)$.

Definition 2.23. A commuting tuple $\left(N_{1}, \ldots, N_{l}\right)$ is called sequentially compatible, if the following holds for each $j$ : 
1. Let $P$ be a point of $D_{\underline{j}}$. Then $\left(N_{1 \mid P}, \ldots, N_{j \mid P}\right)$ is sequentially compatible.

2. We put $N(\underline{j})=\sum_{i \leq j} N_{i \mid D_{j}}$. Then the conjugacy classes of $N(\underline{j})_{\mid Q}$ are independent of $Q \in D_{\underline{j}}^{-}$.

3. Let $W(\underline{j})$ denote the weight filtration of $N(\underline{j})$. Then $(W(\underline{1}), \ldots$, $W(\underline{l}))$ is a compatible sequence of filtrations.

Remark 2.4. When we check whether a commuting tuple $\left(N_{1}, \ldots\right.$, $N_{n}$ ) is sequentially compatible, we only have to check Condition 3 in Definition 2.18 instead of Condition 3 in Definition 2.23.

Definition 2.24. Let $\left(N_{1}, \ldots, N_{l}\right)$ be a sequentially compatible commuting tuple. A frame $\boldsymbol{v}$ is called compatible with $\left(N_{1}, \ldots, N_{l}\right)$, if $\boldsymbol{v}$ is compatible with the sequence $(W(\underline{1}), \ldots, W(\underline{l}))$.

Lemma 2.17. There are decompositions of $E_{\mid D_{\underline{m}}}$ for $1 \leq m \leq l$ :

$$
E_{\mid D_{\underline{m}}}=\bigoplus_{h \in \mathbb{Z}^{m}} \mathcal{K}_{\boldsymbol{h}}
$$

They satisfy the following:

1. For any $\boldsymbol{h} \in \mathbb{Z}^{m}$, we have $\bigcap_{j=1}^{m} W(\underline{j})_{h_{j}}=\bigoplus_{\boldsymbol{k} \in \mathcal{U}(\boldsymbol{h})} \mathcal{K}_{\boldsymbol{k}}$ on $D_{\underline{m}}$. Here $\mathcal{U}(\boldsymbol{h})$ denotes the set of $\boldsymbol{k} \in \mathbb{Z}^{n}$ satisfying $\rho_{j}(\boldsymbol{k})=\sum_{i \leq j} q_{i}(\boldsymbol{k})$ $\leq h_{j}$ for any $1 \leq j \leq m$.

2. We have $\mathcal{K}_{\boldsymbol{h} \mid D_{m+1}}=\bigoplus_{k} \mathcal{K}_{(\boldsymbol{h}, k)}$. Here $(\boldsymbol{h}, k)=\left(h_{1}, \ldots, h_{m}, k\right)$ for $\boldsymbol{h}=\left(h_{1}, \ldots, h_{m}\right)$.

Proof. Since $(W(\underline{1}), \ldots, W(\underline{l}))$ is compatible, we have the compatible splitting $\left\{\overline{\mathcal{K}}_{\boldsymbol{h}} \mid \boldsymbol{h} \in \mathbb{Z}^{m}, m=1, \ldots, l\right\}$. For any $\boldsymbol{h}=\left(h_{1}, \ldots, h_{m}\right) \in \mathbb{Z}^{m}$, we put $\mu(\boldsymbol{h}):=\left(\rho_{1}(\boldsymbol{h}), \rho_{2}(\boldsymbol{h}), \ldots, \rho_{n}(\boldsymbol{h})\right)$. Then we put as follows:

$$
\mathcal{K}_{h}=\overline{\mathcal{K}}_{\mu(h)} .
$$

Then the tuple $\left\{\mathcal{K}_{\boldsymbol{h}} \mid \boldsymbol{h} \in \mathbb{Z}^{m} m=1, \ldots, l\right\}$ has the desired property.

q.e.d.

Definition 2.25. Such tuple $\left\{\mathcal{K}_{\boldsymbol{h}} \mid \boldsymbol{h} \in \mathbb{Z}^{m}, m=1, \ldots, l\right\}$ is called a compatible splitting of the commuting tuple $\left(N_{1}, \ldots, N_{l}\right)$.

Corollary 2.2. Let $E$ and $N_{1}, \ldots, N_{l}$ be as above. We can take a holomorphic frame $\boldsymbol{v}$ of $E$, which is compatible with $\left(N_{1}, \ldots, N_{l}\right)$. 


\subsubsection{Strongly sequential compatibility}

Let $E$ be a holomorphic vector bundle over $\Delta^{n}$. We put $D_{i}:=\left\{\left(z_{1}, \ldots\right.\right.$, $\left.\left.z_{n}\right) \in \Delta^{n} \mid z_{i}=0\right\}$, and $D_{\underline{m}}=\bigcap_{i=1}^{m} D_{i}$. Let $l$ be a number less than $n$. Let $N_{i}$ be an element of $\Gamma\left(D_{i}, \operatorname{End}(E)_{\mid D_{i}}\right)$ for $i=1, \ldots, l$. For any $m \leq l$, we have the nilpotent maps $N_{1 \mid D_{\underline{m}}}, \ldots, N_{m \mid D_{\underline{m}}}$ of $E_{\mid D_{\underline{m}}}$.

Definition 2.26. We say that the tuple $\left(N_{1}, \ldots, N_{l}\right)$ is strongly sequentially compatible, if the following holds:

1. All the assumptions in Definition 2.23 are satisfied.

2. Moreover $\left(N_{1 \mid P}, \ldots, N_{m \mid P}\right)$ is assumed to be strongly sequentially compatible for each $P \in D_{\underline{m}}$.

Lemma 2.18. There are decompositions of $E_{\mid D_{\underline{m}}}$ for $1 \leq m \leq l$ :

$$
E_{\mid D_{\underline{m}}}=\bigoplus_{k \geq 0} \bigoplus_{\boldsymbol{h} \in \mathbb{Z}^{m}} P_{k} \mathcal{K}_{\boldsymbol{h}}
$$

They satisfy the following:

1. For any $\boldsymbol{h} \in \mathbb{Z}^{m}$ and $k \geq 0$, we have

$$
\bigcap_{j=1}^{m} W(\underline{j})_{h_{j}}=\bigoplus_{k \geq 0} \bigoplus_{\boldsymbol{k} \in \mathcal{U}(\boldsymbol{h})} P_{k} \mathcal{K}_{\boldsymbol{k}}
$$

on $D_{\underline{m}}$. Here $\mathcal{U}(\boldsymbol{h})$ denotes the set of $\boldsymbol{k} \in \mathbb{Z}^{n}$ satisfying $\rho_{j}(\boldsymbol{k}) \leq h_{j}$ for any $1 \leq j \leq m$.

2. We have $P_{k} \mathcal{K}_{\boldsymbol{h} \mid D_{m+1}}=\bigoplus_{a} P_{k} \mathcal{K}_{(\boldsymbol{h}, a)}$. Here $(\boldsymbol{h}, a)=\left(h_{1}, \ldots, h_{m}\right.$, a) for $\boldsymbol{h}=\left(h_{1}, \ldots, h_{m}\right)$.

3. $P_{k} \mathcal{K}_{\boldsymbol{h}}=0$ unless $\left|q_{1}(\boldsymbol{h})\right| \leq k$ and $k-q_{1}(\boldsymbol{h})$ is even.

4. When $-k<q_{1}(\boldsymbol{h}) \leq k$, we have $N_{1}\left(P_{k} \mathcal{K}_{\boldsymbol{h}}\right)=P_{k} \mathcal{K}_{\boldsymbol{h}-2 \boldsymbol{\delta}_{1}}$. Here we put $\boldsymbol{h}-2 \boldsymbol{\delta}_{1}=\left(h_{1}-2, h_{2}, \ldots, h_{n}\right)$.

5. When $h_{1}=-k, N_{1}\left(P_{k} \mathcal{K}_{\boldsymbol{h}}\right)=0$.

Proof. On $D_{\underline{1}}$, we have the graded vector space $P_{h} G r_{h}^{(1)}$. On $D_{\underline{j}}$ for $2 \leq j \leq l$, we have the nilpotent morphisms $N_{j}^{(1)}$ of $P_{h} G r_{h}^{(1)}$. Since $\left(N_{2}^{(1)}, \ldots, N_{l}^{(2)}\right)$ is sequentially compatible, we obtain the compatible decompositions $P_{h} G r_{h \mid D_{\underline{m}}}^{(1)}=\bigoplus_{\boldsymbol{k}^{\prime} \in \mathbb{Z}^{m}} \mathcal{K}_{h, \boldsymbol{k}^{\prime}}$ for any $2 \leq m \leq l$. 
Then we construct $P_{k} \mathcal{K}_{\boldsymbol{h}}$ on $D_{\underline{m}}$ by using an induction on $m$. Assume that we already have the decomposition on $D_{m+1}$. Let $\boldsymbol{h}$ be a tuple $\left(h_{1}, \ldots, h_{m}\right)$. We construct $P_{h_{1}} \mathcal{K}_{\boldsymbol{h}}$ on $D_{\underline{m}}$ in the following.

For a tuple $\boldsymbol{h}=\left(h_{1}, \ldots, h_{m}\right)$, we put $\chi(\overline{\boldsymbol{h}}):=\left(h_{1}, h_{2}-h_{1}, h_{3}-\right.$ $\left.h_{2}, \ldots, h_{m}-h_{m-1}\right)$, and $\chi^{\prime}(\boldsymbol{h})=\left(h_{2}-h_{1}, h_{3}-h_{2}, \ldots, h_{m}-h_{m-1}\right)$. Note that $\chi(\boldsymbol{h}) \in \mathcal{U}(\boldsymbol{h})$. If $\boldsymbol{h}^{\prime}=\left(h_{2}-h_{1}, \ldots, h_{m}-h_{1}\right) \in \mathbb{Z}^{m-1}$, then we have $\chi^{\prime}(\boldsymbol{h}) \in \mathcal{U}\left(\boldsymbol{h}^{\prime}\right)$.

By our assumption, $\mathcal{K}_{h_{1}, \chi^{\prime}(\boldsymbol{h})}$ is contained in the image of $P \pi_{\boldsymbol{h}}$ :

$$
\begin{aligned}
& P \pi_{\boldsymbol{h}}\left[\operatorname{Ker}\left(N(\underline{\underline{1}})^{h_{1}+1}\right) \cap \bigcap_{j=2}^{m} W(\underline{j})_{h_{j}}\right] \\
& =\left[P_{h_{1}} G r_{h_{1}}^{(1)} \cap \bigcap_{j=2}^{n} W\left(N^{(1)}(\underline{j})\right)_{h_{j}-h_{1}}\right] \supset P_{h_{1}} \mathcal{K}_{h_{1}, \chi^{\prime}(\boldsymbol{h})} .
\end{aligned}
$$

On $D_{m+1}$, we have $\bigoplus_{k} \mathcal{K}_{(\chi(\boldsymbol{h}), k)}$. By extending it, we can take a subbundle $P_{h_{1}} \mathcal{K}_{\chi(\boldsymbol{h})}$ of $\operatorname{Ker}\left(N(\underline{1})^{h_{1}+1}\right) \bigcap_{j=2}^{m} W(\underline{j})_{h_{j}}$ satisfying the following:

- $P_{h_{1}} \mathcal{K}_{\chi(\boldsymbol{h})}$ is isomorphic to $\mathcal{K}_{h_{1}, \chi^{\prime}(\boldsymbol{h})}$ via the morphism $P \pi_{\boldsymbol{h}}$.

- We have $P_{h_{1}} \mathcal{K}_{\chi(\boldsymbol{h}) \mid D_{\underline{m+1}}}=\bigoplus_{a} P_{h_{1}} \mathcal{K}_{(\chi(\boldsymbol{h}), a)}$.

By an inductive argument, we obtain $P_{h} \mathcal{K}_{\boldsymbol{h}}$ on $D_{m}$ for any $m$ and $\boldsymbol{h} \in$ $\mathbb{Z}^{m}$ such that $h_{1}=h$.

For an integer $m$ such that $0 \leq m \leq h_{1}$, we put as follows:

$$
P_{h_{1}} \mathcal{K}_{\boldsymbol{h}-2 m \delta_{1}}:=N_{1}^{m}\left(P_{h_{1}} \mathcal{K}_{\boldsymbol{h}}\right)
$$

Here we put $\boldsymbol{h}-2 m \boldsymbol{\delta}_{1}=\left(h_{1}-2 m, h_{2}, \ldots, h_{n}\right)$. By our choice, we have $N_{1}^{h_{1}+1}\left(P_{h_{1}} \mathcal{K}_{\boldsymbol{h}}\right)=0$. Then we obtain the desired decomposition. q.e.d.

Definition 2.27. Such tuple $\left\{P_{k} \mathcal{K}_{\boldsymbol{h}} \mid k \geq 0, \boldsymbol{h} \in \mathbb{Z}^{m}, m=1, \ldots, l\right\}$ is called the strongly compatible splitting of $\left(N_{1}, \ldots, N_{l}\right)$.

For any tuple $\boldsymbol{h} \in \mathbb{Z}^{l}$ and $k \geq 0$, we have the number $d(k, \boldsymbol{h}):=$ $\operatorname{rank} P_{k} \mathcal{K}_{\boldsymbol{h}}$. Clearly we have $d(k, \boldsymbol{h})=d(k-2, \boldsymbol{h})$ if $-k<q_{1}(\boldsymbol{h}) \leq k$.

Corollary 2.3. Let $E$ and $\left(N_{1}, \ldots, N_{l}\right)$ be as above. Then we can take a holomorphic frame $\boldsymbol{v}$ of $E$, around the origin $O$ of $\Delta^{n}$, satisfying the following:

1. $\boldsymbol{v}=\left(v_{k, \boldsymbol{h}, \eta} \mid k \geq 0, \boldsymbol{h} \in \mathbb{Z}^{l}, \eta=1, \ldots, d(k, \boldsymbol{h})\right)$. 
2. We have $N_{1}\left(v_{k, \boldsymbol{h}, \eta}\right)=v_{k, \boldsymbol{h}-2 \delta_{1}, \eta}$ when $-k<h_{1} \leq k$, on $D_{\underline{1}}$.

3. $N_{1}\left(v_{k, \boldsymbol{h}, \eta}\right)=0$ if $h_{1}=-k$, on $D_{\underline{1}}$.

4. $\operatorname{deg}^{W}(\underline{j})\left(v_{k, \boldsymbol{h}, \eta}\right)=\sum_{i \leq j} h_{i}$.

5. $\boldsymbol{v}$ is compatible with the sequence $(W(\underline{1}), \ldots, W(\underline{l}))$.

Such frame is called strongly compatible with $\left(N_{1}, \ldots, N_{l}\right)$.

Definition 2.28. Such frame is called a frame strongly compatible with $\left(N_{1}, \ldots, N_{l}\right)$.

Definition 2.29. A tuple $\left(N_{1}, \ldots, N_{l}\right)$ is called of Hodge, if $\left(N_{\sigma(1)}\right.$, $\left.\ldots, N_{\sigma(l)}\right)$ is strongly sequentially compatible for any $\sigma \in \mathfrak{S}_{l}$.

\subsection{Mixed twistor structure}

\subsubsection{Definition}

The harmonic metric can be regarded as a generalization of the variation of polarized Hodge structure. To regard the harmonic metric as a variation of some structure, Simpson introduced the twistor structure.

Definition 2.30 (Simpson [36]). The pure twistor structure and the mixed twistor structure are defined as follows:

1. A holomorphic vector bundle on the projective line $\mathbb{P}^{1}$ is called a pure twistor structure of weight $i$ if it is holomorphically isomorphic to a direct some of the line bundle $\mathcal{O}_{\mathbb{P}^{1}}(i)$.

2. A holomorphic vector bundle $V$ with an ascending filtration $W$ by holomorphic vector subbundles is called a mixed twistor structure if $G r_{i}^{W}$ is a pure twistor structure of weight $i$.

He also introduced the variation of the twistor structure and observed that a harmonic bundle can be regarded as the variation of the pure twistor structure. (See [36] for more detail.)

In the next subsubsections, we explain a method to use the mixed twistor structure in this paper.

\subsubsection{Lower bound of the degree}

We will use the mixed twistor structure to obtain a lower bound of the degree. We consider the following situation. Let $(V, W)$ be a mixed 
twistor structure. Let $L$ be a holomorphic vector subbundle of $V$ with the filtration $W_{L}$. If we have the upper bound of the degree of any nonzero element of $W_{L, l}(L)$, i.e., $\operatorname{deg}^{W}(s) \leq l+a$ for any nonzero $s \in$ $W_{L, l}(L)_{\lambda},\left(\lambda \in \mathbb{P}^{1}\right)$, then we have the inclusion $W_{L, l}(L) \subset W_{l+a}$. We have the following easy lemma.

Lemma 2.19. Let $(V, W)$ be a mixed twistor structure. Let a be an integer. Let $L$ be a holomorphic vector subbundle of $V$ with the filtration $W_{L}$ such that $W_{L, l}(L) \subset W_{l+a}$ and that the first Chern classes $\left.c_{1}\left(G r_{l}^{W_{L}}(L)\right)\right)$ are $(l+a) \cdot \operatorname{rank}\left(G r_{l}^{W_{L}}(L)\right)$ for any $l$.

Then the induced morphisms $G r_{l}^{W_{L}}(L) \longrightarrow G r_{l+a}^{W}$ is an injection of vector bundles, that is, $G r_{l}^{W_{L}}$ naturally gives a subbundle of $G r_{l+a}^{W}$. Moreover $G r_{l}^{W_{L}}(L)$ is a pure twistor of weight $l+a$.

In particular, the degree of any nonzero element $s \in W_{L, l}\left(L_{\lambda}\right)-$ $W_{L, l-1}\left(L_{\lambda}\right)$ is exactly $l+a$.

Proof. Let $b$ be the bottom number of the filtration $W_{L}$. First we consider the bottom part $W_{L, b}$. It is well-known that a holomorphic vector bundle $W_{L, b}$ over $\mathbb{P}^{1}$ is holomorphically a direct sum $\bigoplus_{i} \mathcal{O}(i)^{\oplus n_{i}}$. Assume that $W_{L, b}$ is not a pure twistor structure of weight $b+a$. Since we have the equality $\sum n_{i} i=(b+a) \cdot \operatorname{rank}\left(W_{L, b}\right)$, there is an $i>b+a$ such that $n_{i} \neq 0$. It is easy to see that $\operatorname{Hom}\left(\mathcal{O}(i), W_{b+a}\right)=0$ for any $i>b+a$. Hence we have no injective morphism $W_{L, b} \rightarrow W_{b+a}$, which contradicts the fact that $L$ is a subbundle of $W_{b+a}$. Thus $W_{L, b}$ is a pure twistor structure of weight $b+a$. Moreover the composition $W_{L, b} \longrightarrow W_{b+a} \longrightarrow G r_{b+a}$ is injective of the vector bundles.

We use an induction. We assume that we have proved the claims of the theorem for $W_{L, l}$ for any $l<l_{0}$ and then we prove that the claim for $W_{L, l_{0}}$ holds. We have the morphism $\phi_{l_{0}}: G r_{l_{0}}^{W_{L}} \longrightarrow G r_{l_{0}+a}^{W}$. Assume that $\phi_{l_{0}}$ is not injective. Note that $c_{1}\left(\operatorname{Ker}\left(\phi_{l_{0}}\right)\right)$ is larger than $\left(l_{0}+\right.$ $a) \cdot \operatorname{rank} \operatorname{Ker}\left(\phi_{l_{0}}\right)$. We put $K_{l_{0}-1}:=\pi_{l_{0}-1}^{-1}\left(\operatorname{Ker} \phi_{l_{0}}\right)$, where $\pi_{l_{0}-1}$ denotes the projection $W_{L, l_{0}} \longrightarrow W_{L, l_{0}} / W_{L, l_{0}-1}$. Then we have the morphism $\phi_{l_{0}-1}: K_{l_{0}-1} / W_{L, l_{0}-2} \longrightarrow G r_{l_{0}+a-1}$. We have the nontrivial kernel $\operatorname{Ker}\left(\phi_{l_{0}-1}\right)$ such that $c_{1}\left(\operatorname{Ker}\left(\phi_{l_{0}-1}\right)\right)>\left(l_{0}-1+a\right) \cdot \operatorname{rank}\left(\operatorname{Ker}\left(\phi_{l_{0}-1}\right)\right)$. We put $K_{l_{0}-2}:=\pi_{l_{0}-2}^{-1}\left(\operatorname{Ker} \phi_{l_{0}-1}\right)$, where $\pi_{l_{0}-2}$ denotes the projection $W_{L, l_{0}} \longrightarrow W_{L, l_{0}} / W_{l_{0}-2}$. In general, we denote the projection $W_{L, l_{0}} \longrightarrow$ $W_{L, l_{0}} / W_{L, i}$ by $\pi_{i}$. Inductively, we can construct the vector subbundles $K_{i}$ of $W_{L, l_{0}}$ as follows:

Assume that we have $W_{L, i} \subset K_{i} \subset W_{i+a}$. Then we have the morphism $\phi_{i}: K_{i} / W_{L, i-1} \longrightarrow G r_{i+a}$. We put $K_{i-1}:=$ $\pi_{i-1}^{-1}\left(\phi_{i}\right)$. 
Then we can check that $c_{1}\left(K_{i}\right)>(i+a) \operatorname{rank} K_{i}$. For sufficiently small $i$, we obtain the inequality $0=c_{1}\left(K_{i}\right)>(i+a) \cdot 0=0$. Thus we arrive at the contradiction if we assume that $\phi_{l_{0}}$ is not injective. Thus we obtain the injectivity of the morphism $\phi_{l_{0}}: G r_{L, l_{0}} \longrightarrow G r_{l_{0}+a}$ as a vector bundles, namely $\phi_{l_{0} \mid P}$ is a injection for each point $P \in \mathbb{P}^{1}$. Since $G r_{l_{0}+a}$ is pure twistor of weight $l_{0}+a$, we have the inequality $c_{1}\left(G r_{L, l_{0}}\right) \leq\left(l_{0}+a\right) \operatorname{rank} G r_{l_{0}+a}$, which is in fact equality by assumption. Thus $G r_{L, l_{0}}$ is a pure twistor of weight $l_{0}+a$.

q.e.d.

Remark 2.5. It is remarkable that we obtain the lower bound of degree from some topological information, that is, Chern class.

\subsubsection{Morphism of mixed twistors}

Definition 2.31. Let $\left(V^{(i)}, W^{(i)}\right)(i=1,2)$ be mixed twistors. A morphism of mixed twistors are the morphism of locally free coherent sheaves $V^{(1)} \longrightarrow V^{(2)}$ preserving the filtrations.

Let $f$ be a morphism of locally free coherent sheaves $V^{(1)} \longrightarrow V^{(2)}$. We have the morphism $f_{\mid P}: V_{\mid P}^{(1)} \longrightarrow V_{\mid P}^{(2)}$ for any $P \in \mathbb{P}^{1}$. Then the rank of $f_{\mid P}$ are not constant, in general. However, when $f$ is a morphism of mixed twistors, then the rank of $f_{\mid P}$ is constant, as we will see in the following lemma.

Lemma 2.20. Let $\left(V^{(i)}, W^{(i)}\right)(i=1,2)$ be mixed twistors, and $f$ be a morphism of mixed twistors.

1. The rank of $f_{\mid P}$ is constant, and thus the $\operatorname{Cok}(f)$ is locally free. Hence $\left\{\operatorname{Ker}(f)_{\mid P} \mid P \in \mathbb{P}^{1}\right\}$ and $\left\{\operatorname{Im}(f)_{\mid P} \mid P \in \mathbb{P}^{1}\right\}$ form subbundles of $V^{(1)}$ and $V^{(2)}$ respectively.

2. We put $W_{l}(\operatorname{Ker}(f))=W_{l}^{(1)} \cap \operatorname{Ker}(f)$. The filtration $W$. $(\operatorname{Ker}(f))$ induces the mixed twistor structure to $\operatorname{Ker}(f)$.

3. We put $W_{l}(\operatorname{Cok}(f))=\pi\left(W_{l}^{(2)}\right)$, where $\pi$ denotes the projection $V^{(2)} \longrightarrow \operatorname{Cok}(f)$. Then the filtration $W .(\operatorname{Cok}(f))$ gives the mixed twistor structure to $\operatorname{Cok}(f)$.

4. We have the equality $f\left(W_{l}^{(1)}\right)=\operatorname{Im}(f) \cap W_{l}^{(2)}$. We put $W_{l}(\operatorname{Im}(f))$ $=f\left(W_{l}^{(2)}\right)$, and then the filtration $W \cdot(\operatorname{Im}(f))$ gives the mixed twistor structure to $\operatorname{Im}(f)$. 
Proof. Note that $W_{l}^{(i)}(i=1,2)$ are naturally mixed twistor structures, and that we have the morphism $f_{l}: W_{l}^{(1)} \longrightarrow W_{l}^{(2)}$ of the mixed twistors. Thus we can use the induction on $l$.

Assume that $l$ is the bottom number $b_{1}$ of $W^{(1)}$. We have the morphism $f_{b_{1}}: W_{b_{1}}^{(1)} \longrightarrow W_{b_{1}}^{(2)}$ and $W_{b_{1}}^{(1)}$ is isomorphic to a direct sum of $\mathcal{O}\left(b_{1}\right)$. We denote the projection $W_{b_{1}}^{(2)} \longrightarrow G r_{b_{1}}^{(2)}$ by $\pi_{b_{1}}^{(2)}$. Then we obtain the following morphisms

$$
W_{b_{1}}^{(1)} \stackrel{f_{b_{1}}}{\longrightarrow} W_{b_{1}}^{(2)} \stackrel{\pi_{b_{1}}^{(2)}}{\longrightarrow} G r_{b_{1}}^{(2)}
$$

We have the composite $G r_{b_{1}}(f):=\pi_{b_{1}}^{(2)} \circ f_{b_{1}}$. Since $W_{b_{1}}^{(1)}$ and $G r_{b_{1}}^{(2)}$ are pure twistor of weight $b_{1}$, it is easy to see the following:

- The ranks of $G r_{b_{1}}(f)_{\mid P}$ are independent of $P \in \mathbb{P}^{1}$.

- The kernel, the image and the cokernel of $G r_{b_{1}}(f)$ are pure twistors of weight $b_{1}$. The kernel is a subbundle of $W_{b_{1}}^{(1)}$.

We have the naturally defined morphism $\operatorname{Ker}\left(G r_{b_{1}}(f)\right) \longrightarrow W_{b_{1}-1}^{(2)}$. Then it is easy to see that the morphism is in fact 0 . Thus $\operatorname{Ker}\left(G r_{b_{1}}(f)\right)$ and $\operatorname{Ker}\left(f_{b_{1}}\right)$ are same. We also obtain the following exact sequence:

$$
0 \longrightarrow W_{b_{1}-1}^{(2)} \longrightarrow \operatorname{Cok}\left(f_{b_{1}}\right) \longrightarrow \operatorname{Cok}\left(G r\left(f_{b_{1}}\right)\right) \longrightarrow 0 .
$$

Thus the $\operatorname{Cok}\left(f_{b_{1}}\right)$ is locally free, and the ranks of $f_{b_{1} \mid P}$ are independent of $P \in \mathbb{P}^{1}$. We also know that the image $\operatorname{Im}\left(f_{b_{1}}\right)$ is a subbundle of $W_{b_{1}}$, and $\operatorname{Im}\left(f_{b_{1}}\right)$ is a pure twistor of weight $b_{1}$. Thus we can show the claim 2.20. In all, we obtain the claims in the case that $l$ is the bottom number of $W^{(1)}$.

We assume that the claims hold for $f_{l-1}: W_{l-1}^{(1)} \longrightarrow W_{l-1}^{(2)}$, and we will prove that the claims hold for $f_{l}: W_{l}^{(1)} \longrightarrow W_{l}^{(2)}$. Since $f_{l}$ preserves the filtration, we have the natural morphism $G r_{l}(f): G r_{l}^{(1)} \longrightarrow G r_{l}^{(2)}$. Because $G r_{l}^{(i)}$ are pure twistors of weight $l$, it is easy to see the following:

- The ranks of $G r_{l}(f)_{\mid P}$ are independent of $P \in \mathbb{P}^{1}$.

- The kernel, the image and the cokernel of $G r_{l}(f)$ is pure twistors of weight $l$. The kernel is a subbundle of $G r_{l}^{(1)}$, and the image is a subbundle of $G r_{l}^{(2)}$. 
We have the natural morphism $\phi: \operatorname{Ker}\left(f_{l}\right) \longrightarrow \operatorname{Ker}\left(G r_{l}(f)\right)$. By an easy diagramm chasing, we obtain the injection $\operatorname{Cok}(\phi) \longrightarrow \operatorname{Cok}\left(f_{l-1}\right)$ of coherent sheaves. By our assumption, $\operatorname{Cok}\left(f_{l-1}\right)$ is mixed twistor, such that $G r_{l}\left(\operatorname{Cok}\left(f_{l-1}\right)\right)=0$. On the other hand, $\operatorname{Cok}(\phi)$ is a quotient of a pure twistor $\operatorname{Ker}\left(G r_{l}(f)\right)$ of weight $l$. Thus the morphism $\operatorname{Cok}(\phi) \longrightarrow$ $\operatorname{Cok}\left(f_{l-1}\right)$ must be 0 , in other words, $\phi$ must be surjective. Thus we obtain the exact sequence:

$$
0 \longrightarrow \operatorname{Ker}\left(f_{l-1}\right) \longrightarrow \operatorname{Ker}\left(f_{l}\right) \longrightarrow \operatorname{Ker}\left(G r_{l}(f)\right) \longrightarrow 0 .
$$

It implies the assertions for $\operatorname{Ker}\left(f_{l}\right)$. We also obtain the exact sequences:

$$
\begin{gathered}
0 \longrightarrow \operatorname{Im}\left(f_{l-1}\right) \longrightarrow \operatorname{Im}\left(f_{l}\right) \longrightarrow \operatorname{Im}\left(G r_{l}(f)\right) \longrightarrow 0 \\
0 \longrightarrow \operatorname{Cok}\left(f_{l-1}\right) \longrightarrow \operatorname{Cok}\left(f_{l}\right) \longrightarrow \operatorname{Cok}\left(G r_{l}(f)\right) \longrightarrow 0 .
\end{gathered}
$$

It implies the assertions for $\operatorname{Im}\left(f_{l}\right)$ and $\operatorname{Cok}\left(f_{l}\right)$. Thus we are done. q.e.d.

Remark 2.6. Note that the property 4 is remarkable. If we consider a morphism of filtered vector spaces $\left(V^{(1)}, W^{(1)}\right) \longrightarrow\left(V^{(2)}, W^{(2)}\right)$, the properties 1, 2 and 3 obviously holds. However 4 does not hold, in general.

Let $(V, W)$ be a mixed twistor, and $a$ be an integer. Then we have the naturally defined mixed twistor of $V \otimes \mathcal{O}(a)$, as follows:

$$
W_{l}(V \otimes \mathcal{O}(a))=W_{l-a}(V) \otimes \mathcal{O}(a) .
$$

It is easy to check that $(V \otimes \mathcal{O}(a), W)$ gives a mixed twistor.

Let $f: V \longrightarrow V \otimes \mathcal{O}(a)$ be a morphism of locally free coherent sheaves. Then $f_{\mid P}: V \longrightarrow V$ is determined as an element of $\mathbb{P}\left(M(r)^{\vee}\right)$. Here $r$ denotes a rank of $V$ and $M(r)^{\vee}$ denotes the dual space of the vector space of $r$-matrices. When $f$ is nilpotent, then it induces the filtration $W\left(f_{\mid P}\right)$ on the fiber $V_{\mid P}$ for any point $P \in \mathbb{P}^{1}$.

Lemma 2.21. Let $(V, W)$ be a mixed twistor and $f:(V, W) \longrightarrow$ $(V \otimes \mathcal{O}(2), W)$ be a nilpotent morphism of mixed twistor. Then the conjugacy classes of the endomorphisms $f_{\mid P}$ are independent of $P \in \mathbb{P}^{1}$.

Proof. Let $\eta$ be a generic point of $\mathbb{P}^{1}$. Then we have the filtration $W\left(f_{\mid \eta}\right)$ of $V_{\mid \eta}$ induced by the nilpotent maps $f_{\mid \eta}$. Let $b$ be the bottom number of the filtration $W\left(f_{\mid \eta}\right)$. We use the induction on $b$.

When $b=0$, then $f_{\mid \eta}=0$. Thus $f=0$. Thus we have nothing to prove. Assume that we have proved the claim in the case $b>b_{0}$, and 
we will prove the claim in the case $b=b_{0}$. Note that the bottom part of the filtration $W\left(f_{\mid \eta}\right)$ is same as $\operatorname{Im}\left(f_{\mid \eta}^{-b_{0}}\right)$ in $V_{\mid \eta}$, and $W_{-b_{0}-1}\left(f_{\mid \eta}\right)$ is same as $\operatorname{Ker}\left(f_{\mid \eta}^{-b_{0}}\right)$. We also have $f^{-b_{0}+1}=0$.

We have the morphism $f^{-b_{0}}: V \longrightarrow V \otimes \mathcal{O}\left(-2 b_{0}\right)$, which is a morphism of mixed twistors. Then $\operatorname{Ker}\left(f^{-b_{0}}\right)$ and $\operatorname{Im}\left(f^{-b_{0}}\right)$ are subbundles of $V$ and $V \otimes \mathcal{O}\left(-2 b_{0}\right)$, and they have the naturally induced mixed twistors. We put as follows:

$$
V^{\prime}=\frac{\operatorname{Ker}\left(f^{-b_{0}}\right)}{\operatorname{Im}\left(f^{-b_{0}}\right) \otimes \mathcal{O}\left(2 b_{0}\right)}
$$

Then it has the naturally induced mixed twistor structure.

We have the naturally induced morphism $\tilde{f}: V^{\prime} \longrightarrow V^{\prime} \otimes \mathcal{O}(2)$. We can apply the claim for $b<b_{0}$ to $\widetilde{f}$, and thus the conjugacy class of $\widetilde{f}_{\mid P}$ are independent of $P \in \mathbb{P}^{1}$. Then we obtain the independence of the conjugacy class of $\tilde{f}_{\mid P}$.

q.e.d.

\subsubsection{Sub mixed twistors}

Let $(V, W)$ be mixed a twistor. Let $V_{1}$ be a subbundle of $V$. Then we obtain the filtration of $V_{1}$ by the coherent subsheaves $W_{h} \cap V_{1}$.

Definition 2.32. We say that $V_{1}$ is a sub mixed twistor of $(V, W)$ if the filtration $\left\{W_{h} \cap V_{1} \mid h \in \mathbb{Z}\right\}$ gives a mixed twistor structure.

We have already shown the following:

Lemma 2.22. Let $f:\left(V_{1}, W_{1}\right) \longrightarrow\left(V_{2}, W_{2}\right)$ be a morphism of mixed twistors. Then the kernel and image are sub mixed twistors of $\left(V_{1}, W_{1}\right)$ and $\left(V_{2}, W_{2}\right)$ respectively.

Lemma 2.23. Let $(V, W)$ be a mixed twistor, and $V_{i}(i=1,2)$ be sub mixed twistors. Then $V_{1}+V_{2}$ and $V_{1} \cap V_{2}$ are also sub mixed twistors.

Proof. We can regard $V_{1} \cap V_{2}$ is the kernel of the morphism of mixed twistors $V_{1} \oplus V_{2} \longrightarrow V$. We can regard $V_{1}+V_{2}$ is the image of the morphism.

q.e.d.

Let $N:(V, W) \longrightarrow(V, W) \otimes \mathcal{O}(2)$ be a morphism of mixed twistors. Since the conjugacy classes of $N$ are independent of $\lambda \in \mathbb{P}^{1}$, we obtain the weight filtration $W(N)$ of $N$ by vector subbundles. The following lemma is easy to see. 
Lemma 2.24. For any $h$, the vector subbundle $W(N)_{h}$ is a sub mixed twistor.

Let $N_{i}:(V, W) \longrightarrow(V, W) \otimes \mathcal{O}(2)$ be a morphism of mixed twistors for $i=1, \ldots, n$.

Lemma 2.25. For any tuple $\boldsymbol{h}=\left(h_{1}, \ldots, h_{n}\right) \in \mathbb{Z}^{n}, \bigcap_{j=1}^{n} W\left(N_{i}\right)_{h_{j}}$ is sub mixed twistor of $(V, W)$.

\subsubsection{Commuting tuple of nilpotent maps}

Let $V$ be a vector bundle over $\mathbb{P}^{1}$. Let $\left(N_{1}, \ldots, N_{n}\right)$ be a commuting tuple of nilpotent morphisms $V \longrightarrow V \otimes \mathcal{O}_{\mathbb{P}^{1}}(2)$.

Proposition 2.3. Assume the following:

1. The weight filtration $W(\underline{n})$ of $N(\underline{n})$ is a mixed twistor.

2. $N(\underline{j}):(V, W(\underline{n})) \longrightarrow(V, W(\underline{n})) \otimes \mathcal{O}(2)(1 \leq j \leq n)$ gives a morphism of mixed twistor structure.

3. $\left(N_{1 \mid \lambda}, \ldots, N_{n-1 \mid \lambda}, N_{n \mid \lambda}\right)$ is sequentially compatible at any point $\lambda \in \mathbb{P}^{1}$.

4. $\left(N_{1 \mid \lambda}, \ldots, N_{n-1 \mid \lambda}\right)$ is strongly sequentially compatible at any point $\lambda \in \mathbb{P}^{1}$.

Then $\left(N_{1 \mid \lambda}, \ldots, N_{n-1 \mid \lambda}, N_{n \mid \lambda}\right)$ is strongly sequentially compatible at any $\lambda \in \mathbb{P}^{1}$.

Proof. First we note that we have $W^{(1)}(\underline{j})_{a+l} \cap G r_{a}^{(1)}=W\left(N^{(1)}(\underline{j})\right)_{l} \cap$ $G r_{a}^{(1)}$, by Assumption 3. By Assumption $4, P \pi_{\boldsymbol{h}}$ induces the following isomorphism for any $\boldsymbol{h} \in \mathbb{Z}^{n-1}$ :

$$
P \pi_{\boldsymbol{h}}: \operatorname{Ker}\left(N(\underline{1})^{h_{1}+1}\right) \cap \bigcap_{j=2}^{n-1} W(\underline{j})_{h_{j}} \longrightarrow P_{h_{1}} G r_{h_{1}}^{(1)} \cap \bigcap_{j=2}^{n-1} W^{(1)}(\underline{j})_{h_{j}}
$$

Due to Conditions 1 and 2, the filtrations $W(\underline{n})$ and $W^{(1)}(\underline{n})$ induce the mixed twistor structures on the both sides of (8). Due to Condition 3, the morphism $P \pi_{\boldsymbol{h}}$ preserves the mixed twistor structures. Thus we can conclude that the morphism $P \pi_{h}$ gives a surjection of the filtered vector bundles over $\mathbb{P}^{1}$. It implies that $\left(N_{1 \mid \lambda}, \ldots, N_{n-1 \mid \lambda}, N_{n \mid \lambda}\right)$ is strongly sequentially compatible at any $\lambda \in \mathbb{P}^{1}$. 


\section{Preliminary for harmonic bundles}

\subsection{Harmonic bundles and deformed holomorphic bun- dles}

\subsection{1 harmonic bundles}

Let $X$ be a complex manifold. Let $\left(E, \bar{\partial}_{E}\right)$ be a holomorphic bundle. Here $E$ denotes a $C^{\infty}$-vector bundle and $\bar{\partial}_{E}$ denotes an operator $\bar{\partial}_{E}$ : $C^{\infty}(X, E) \longrightarrow C^{\infty}\left(X, E \otimes \Omega_{X}^{0,1}\right)$, such that $\left(\bar{\partial}_{E}\right)^{2}=0$ and that $\bar{\partial}_{E}(f v)=$ $\bar{\partial}(f) \cdot v+f \cdot \bar{\partial}_{E}(v)$ for any $f \in C^{\infty}(X)$ and $v \in C^{\infty}(X, E)$. Let $h$ be a hermitian metric of $E$. We denote the inner product of $h$ by $(\cdot, \cdot)_{h}$. We often omit $h$ if there is no confusion. For a holomorphic vector bundle $\left(E, \bar{\partial}_{E}\right)$ with a hermitian metric, we obtain $\partial_{E}: C^{\infty}(X, E) \longrightarrow$ $C^{\infty}\left(X, E \otimes \Omega^{1,0}\right)$ satisfying $\bar{\partial}(f, g)_{h}=\left(\bar{\partial}_{E}(f), g\right)_{h}+\left(f, \partial_{E}(g)\right)_{h}$. We denote the curvature of the unitary connection $\partial_{E}+\bar{\partial}_{E}$ by $R\left(\partial_{E}+\bar{\partial}_{E}\right)$. We often use the notation $R(h)$, if the holomorphic structure is fixed.

Let $\theta$ be a section of $C^{\infty}\left(X, \operatorname{End}(E) \otimes \Omega^{1,0}\right)$. It is called a (holomorphic) Higgs field if $\bar{\partial}_{E} \theta=0$ and $\theta \wedge \theta=0$. The tuple $\left(E, \bar{\partial}_{E}, h\right)$ is called a Higgs bundle.

We have the adjoint of $\theta$ with respect to $h$, which we denote by $\theta^{\dagger}$, namely $(\theta \cdot f, g)_{h}=\left(f, \theta^{\dagger} \cdot g\right)_{h}$. Then $\theta^{\dagger}$ is an element of $C^{\infty}(X, \operatorname{End}(E) \otimes$ $\left.\Omega^{0,1}\right)$ satisfying $\partial_{E}\left(\theta^{\dagger}\right)=0$ and $\theta^{\dagger} \wedge \theta^{\dagger}=0$.

From a Higgs bundle $\left(E, \bar{\partial}_{E}, \theta\right)$ with a hermitian metric $h$, we obtain the following connection:

$$
\mathbb{D}^{1}:=\bar{\partial}_{E}+\partial_{E}+\theta+\theta^{\dagger}: C^{\infty}(X, E) \longrightarrow C^{\infty}\left(X, E \otimes \Omega_{X}^{1}\right) .
$$

Definition 3.1. A tuple $\left(E, \bar{\partial}_{E}, h, \theta\right)$ is called a harmonic bundle, if $\mathbb{D}^{1}$ is flat, namely $\mathbb{D}^{1} \circ \mathbb{D}^{1}=0$.

Remark 3.1. Probably, such object should be called a pluriharmonic bundle. But we use 'harmonic bundle' for simplicity.

The condition $\mathbb{D}^{1} \circ \mathbb{D}^{1}=0$ is equivalent to the following:

$$
\left(\bar{\partial}_{E}+\theta^{\dagger}\right)^{2}=\left(\partial_{E}+\theta\right)^{2}=R\left(\partial_{E}+\bar{\partial}_{E}\right)+\theta \wedge \theta^{\dagger}+\theta^{\dagger} \wedge \theta=0 .
$$

Lemma 3.1 (Corlette, Simpson, [37]). Let $\left(E, \bar{\partial}_{E}, h, \theta\right)$ be a harmonic bundle. Then we have $\partial_{E}(\theta)=\bar{\partial}_{E}\left(\theta^{\dagger}\right)=0$.

Proof. We know that $\partial_{E}^{2}=\theta^{2}=\left(\partial_{E}+\theta\right)^{2}=0$. It implies that $\partial_{E}(\theta)=0$. Similarly we obtain the equality $\bar{\partial}_{E}\left(\theta^{\dagger}\right)=0$. $\quad$ q.e.d. 


\subsubsection{The deformed holomorphic bundle}

Let $\left(E, \bar{\partial}_{E}, h, \theta\right)$ be a harmonic bundle over $X$. We denote $\mathbf{C}_{\lambda} \times X$ by $\mathcal{X}$. We denote the projection $\mathbf{C}_{\lambda} \times X \longrightarrow X$ by $p_{\lambda}$. We have the $C^{\infty}$ bundle $p_{\lambda}^{-1}(E)$ over $\mathcal{X}$. We have the operator $d^{\prime \prime}: C^{\infty}\left(\mathcal{X}, p_{\lambda}^{-1}(E)\right) \longrightarrow$ $C^{\infty}\left(\mathcal{X}, p_{\lambda}^{-1}(E) \otimes \Omega_{\mathcal{X}}^{0,1}\right)$.

$$
d^{\prime \prime}:=\bar{\partial}_{E}+\lambda \cdot \theta^{\dagger}+\bar{\partial}_{\lambda}
$$

Lemma 3.2 (Simpson, [36]). The operator d" gives a holomorphic structure of $p_{\lambda}^{-1}(E)$.

Proof. We only have to see that $d^{\prime \prime} \circ d^{\prime \prime}=0$, which follows from $\bar{\partial}_{E}\left(\theta^{\dagger}\right)=0$.

q.e.d.

The holomorphic bundle $\left(p_{\lambda}^{-1}(E), d^{\prime \prime}\right)$ is denoted by $\mathcal{E}$, which we call the deformed holomorphic bundle. We have the pull back of the hermitian metric $h$. The metric connection is given by the following:

$$
d_{\lambda}+\bar{\partial}_{E}+\partial_{E}+\lambda \cdot \theta^{\dagger}-\bar{\lambda} \cdot \theta
$$

The curvature of the metric is as follows:

$$
-d \bar{\lambda} \cdot \theta+d \lambda \cdot \theta^{\dagger}+R\left(\bar{\partial}_{E}+\partial_{E}\right)-|\lambda|^{2}\left[\theta, \theta^{\dagger}\right] .
$$

We put as follows:

$$
\mathcal{X}^{\lambda}:=\{\lambda\} \times X, \quad \mathcal{X}^{\sharp}:=\mathbf{C}_{\lambda}^{*} \times X .
$$

The restrictions $\left(\mathcal{E}, d^{\prime \prime}\right)_{\mid \mathcal{X}^{\lambda}}$ and $\left(\mathcal{E}, d^{\prime \prime}\right)_{\mid \mathcal{X}^{\sharp}}$ are denoted by $\left(\mathcal{E}^{\lambda}, d^{\prime \prime \lambda}\right)$ and $\left(\mathcal{E}^{\sharp}, d^{\prime \prime \sharp}\right)$.

\subsubsection{The $\lambda$-connection}

Let $\left(E, \bar{\partial}_{E}\right)$ be a holomorphic bundle. Let $\lambda$ be a complex number. In general, an operator $\nabla^{\lambda}: C^{\infty}(X, E) \longrightarrow C^{\infty}\left(X, E \otimes \Omega^{1}\right)$ is called a $\lambda$ connection if the following holds for any $f \in C^{\infty}(X)$ and $v \in C^{\infty}(X, E)$ :

$$
\nabla^{\lambda}(f \cdot v)=(\lambda \partial(f)+\bar{\partial}(f)) \cdot v+f \cdot \nabla^{\lambda}(v) .
$$

It is called holomorphic if the $(0,1)$-part is same as $\bar{\partial}_{E}$. It is called flat if $\nabla^{\lambda} \circ \nabla^{\lambda}=0$.

It is easy to see that a flat holomorphic 0-connection is equivalent to a pair of holomorphic structure and a holomorphic Higgs field. And a 
flat holomorphic 1-connection is equivalent to an ordinary holomorphic flat connection.

Let $\left(E, \bar{\partial}_{E}, \theta, h\right)$ be a harmonic bundle over $X$. Then we have the operator $\mathbb{D}^{\lambda}: C^{\infty}(X, E) \longrightarrow C^{\infty}\left(X, E \otimes \Omega_{X}^{1}\right)$ defined as follows:

$$
\mathbb{D}^{\lambda}=\bar{\partial}_{E}+\theta+\lambda\left(\partial_{E}+\theta^{\dagger}\right)
$$

Recall that we have the holomorphic bundle $\mathcal{E}^{\lambda}$ on $\mathcal{X}^{\lambda}$, whose holomorphic structure is given by $\bar{\partial}_{E}+\lambda \theta^{\dagger}$.

Lemma 3.3. The operator $\mathbb{D}^{\lambda}$ is a flat holomorphic $\lambda$-connection of $\mathcal{E}^{\lambda}$.

Proof. It is clear from the definition that $\mathbb{D}^{\lambda}$ gives a holomorphic $\lambda$-connection of $\mathcal{E}^{\lambda}$. We have the following equality:

$$
\mathbb{D}^{\lambda} \circ \mathbb{D}^{\lambda}=\left(\bar{\partial}_{E}+\lambda \cdot \theta^{\dagger}\right)^{2}+\left(\lambda \partial_{E}+\theta\right)^{2}+\lambda \cdot\left(R\left(\partial_{E}+\bar{\partial}_{E}\right)+\left[\theta, \theta^{\dagger}\right]\right) .
$$

Since we have $\partial(\theta)=\bar{\partial}_{E}\left(\theta^{\dagger}\right)=0$, we can obtain the desired flatness.

q.e.d.

The $\mathbb{D}^{\lambda}$ is called the $\lambda$-connection associated with the harmonic bundle $\left(E, \bar{\partial}_{E}, \theta, h\right)$. We have the operator $\mathbb{D}: C^{\infty}(\mathcal{X}, \mathcal{E}) \longrightarrow C^{\infty}(\mathcal{X}, \mathcal{E} \otimes$ $\left.p_{\lambda}^{*} \Omega_{X}^{1}\right)$ defined by $\mathbb{D}=\bar{\partial}_{E}+\theta+\lambda\left(\partial_{E}+\theta^{\dagger}\right)$. The operator $\mathbb{D}$ is also called the $\lambda$-connection associated with $\left(E, \bar{\partial}_{E}, \theta, h\right)$.

Note that $\mathbb{D}$ and $\bar{\partial}_{\lambda}$ are commutative. Thus $\mathbb{D}(v)$ is holomorphic if $v$ is holomorphic section of $\mathcal{E}$. Let $\boldsymbol{v}$ be a holomorphic frame of $\mathcal{E}$ on an open subset $U$ of $\mathcal{X}$. Then the $\lambda$-connection form $\mathcal{A}=\left(\mathcal{A}_{i j}\right)$ is defined by the following relation:

$$
\mathbb{D} v_{j}=\sum_{i} \mathcal{A}_{i j} \cdot v_{i}
$$

Obviously $\mathcal{A}_{i j}$ are holomorphic sections of $p_{\lambda}^{*} \Omega_{X}^{1,0}$, i.e., $\mathcal{A}$ is an element of $\Gamma\left(U, M(r) \otimes p_{\lambda}^{-1} \Omega_{X}^{1,0}\right)$, where $r$ is a rank of $E$. We describe as $\mathbb{D} \boldsymbol{v}=\boldsymbol{v} \cdot \mathcal{A}$.

\subsubsection{The associated flat connections}

For any $\lambda \neq 0$, we have the holomorphic flat connection of $\mathcal{X}^{\lambda}$ :

$$
\mathbb{D}^{\lambda, f}:=\bar{\partial}_{E}+\lambda \cdot \theta^{\dagger}+\partial_{E}+\lambda^{-1} \theta
$$

Again the flatness follows from the equalities $\partial_{E}(\theta)=\bar{\partial}_{E}\left(\theta^{\dagger}\right)=0$. 
We have the operator $\mathbb{D}^{f}:=\bar{\partial}_{E}+\partial_{E}+\lambda \theta^{\dagger}+\lambda^{-1} \theta: C^{\infty}\left(\mathcal{X}^{\sharp}, \mathcal{E}^{\sharp}\right) \longrightarrow$ $C^{\infty}\left(\mathcal{X}^{\sharp}, \mathcal{E}^{\sharp} \otimes p_{\lambda}^{*} \Omega_{X}^{1}\right)$. We call it the associated family of the flat connections.

Let $\boldsymbol{v}$ be a holomorphic frame of $\mathcal{E}^{\sharp}$ on some open subset $U$ of $\mathcal{X}^{\sharp}$. Then we obtain the holomorphic section $\mathcal{A}^{f}=\left(\mathcal{A}_{i j}^{f}\right)$ of $\Gamma(U, M(r) \otimes$ $\left.p_{\lambda}^{*} \Omega_{X}^{1,0}\right)$ defined as follows:

$$
\mathbb{D}^{f} v_{j}=\sum_{i} \mathcal{A}_{i j}^{f} \cdot v_{i}, \quad \text { i.e., } \quad \mathbb{D}^{f} \boldsymbol{v}=\boldsymbol{v} \cdot \mathcal{A}^{f} .
$$

Lemma 3.4. We have the relation $\mathcal{A}^{f}=\lambda^{-1} \cdot \mathcal{A}$.

Proof. The $(0,1)$-parts of $\mathbb{D}^{f}$ and $\mathbb{D}$ are same, we have the following relation between the $(1,0)$-parts of $\mathbb{D}^{f}$ and $\mathbb{D}$ :

$$
\mathbb{D}^{f(1,0)}=\partial_{E}+\lambda^{-1} \theta=\lambda^{-1}\left(\lambda \cdot \partial_{E}+\theta\right)=\lambda^{-1} \cdot \mathbb{D}^{(1,0)} .
$$

Thus we are done.

q.e.d.

\subsubsection{Conjugate}

We denote the conjugate of $X$ by $X^{\dagger}$. Namely $X^{\dagger}$ denotes the complex manifold whose underlying $C^{\infty}$-manifold is same as $X$ and whose holomorphic structure is given by $\partial$. If $\left(E, \bar{\partial}_{E}\right)$ is a holomorphic bundle over $X$, then $\left(E, \partial_{E}\right)$ is a holomorphic bundle over $X^{\dagger}$. If $\theta$ is a holomorphic Higgs field of $\left(E, \bar{\partial}_{E}\right)$ over $X$, then $\theta^{\dagger}$ is a holomorphic Higgs field of $\left(E, \partial_{E}\right)$ over $X^{\dagger}$.

Let $\left(E, \bar{\partial}_{E}, \theta, h\right)$ be a harmonic bundle over $X$. Then the conjugate $\left(E, \partial_{E}, h, \theta^{\dagger}\right)$ is a harmonic bundle over $X^{\dagger}$. We put $\mathcal{X}^{\dagger}:=\mathbf{C}_{\mu} \times X^{\dagger}$. We denote the projection $\mathcal{X}^{\dagger} \longrightarrow X^{\dagger}$ by $p_{\mu}^{\dagger}$. From a harmonic bundle $\left(E, \partial_{E}, h, \theta^{\dagger}\right)$ over $X^{\dagger}$, we obtain the deformed holomorphic bundle $\left(\mathcal{E}^{\dagger}, d^{\prime \prime} \dagger\right)$ over $\mathcal{X}^{\dagger}$. Under the identification of $X$ and $X^{\dagger}$, the underlying $C^{\infty}$-bundle of $\mathcal{E}^{\dagger}$ is $p_{\mu}^{\dagger-1}(E)$, and the holomorphic structure $d^{\prime \prime \dagger}$ is given by the operator $\partial_{E}+\mu \cdot \theta+\bar{\partial}_{\mu}$.

We put $\mathcal{X}^{\dagger \mu}=\{\mu\} \times X^{\dagger}$ and $\mathcal{X}^{\dagger \sharp}=\mathbf{C}_{\mu}^{*} \times X$. The restrictions of $\left(\mathcal{E}^{\dagger}, d^{\prime \prime \dagger}\right)$ to $\mathcal{X}^{\dagger \mu}$ and $\mathcal{X}^{\dagger \sharp}$ are denoted by $\left(\mathcal{E}^{\dagger \mu}, d^{\prime \prime \mu}\right)$ and $\left(\mathcal{E}^{\dagger \sharp}, d^{\prime \prime \sharp}\right)$. The operator $d^{\prime \prime} \mu$ is same as $\partial_{E}+\mu \cdot \theta$.

We have the associated $\mu$-connections, which we denote by $\mathbb{D}^{\dagger} \mu$. Namely we have the following operator:

$$
\begin{aligned}
\mathbb{D}^{\dagger \mu}:=\partial_{E}+\theta^{\dagger}+\mu \cdot\left(\bar{\partial}_{E}+\theta\right): C^{\infty}\left(\mathcal{X}^{\dagger \mu}, \mathcal{E}^{\dagger \mu}\right) & \\
& \longrightarrow C^{\infty}\left(\mathcal{X}^{\dagger \mu}, \mathcal{E}^{\dagger \mu} \otimes \Omega_{\mathcal{X}^{\dagger \mu}}^{1}\right) .
\end{aligned}
$$


We have the following operator, which we also call the $\mu$-connection:

$$
\begin{aligned}
\mathbb{D}^{\dagger}:=\partial_{E}+\theta^{\dagger}+\mu \cdot\left(\bar{\partial}_{E}+\theta\right): C^{\infty}\left(\mathcal{X}^{\dagger}, \mathcal{E}^{\dagger}\right) & \\
\longrightarrow & C^{\infty}\left(\mathcal{X}^{\dagger}, \mathcal{E}^{\dagger} \otimes p_{\mu}^{\dagger *} \Omega_{X^{\dagger}}^{1}\right) .
\end{aligned}
$$

For any $\mu \neq 0$, The associated flat connections are given as follows:

$$
\begin{aligned}
\mathbb{D}^{\dagger \mu f}:=\partial_{E}+\mu \cdot \theta+\bar{\partial}_{E}+\mu^{-1} \theta^{\dagger}: C^{\infty} & \left(\mathcal{X}^{\dagger \mu}, \mathcal{E}^{\dagger \mu}\right) \\
& \longrightarrow C^{\infty}\left(\mathcal{X}^{\dagger \mu}, \mathcal{E}^{\dagger \mu} \otimes \Omega_{\mathcal{X}^{\dagger \mu}}^{1}\right) .
\end{aligned}
$$

We have the family of the flat connections:

$$
\begin{aligned}
\mathbb{D}^{\dagger f}:=\partial_{E}+\mu \cdot \theta+\bar{\partial}_{E}+\mu^{-1} \theta^{\dagger}: C^{\infty}\left(\mathcal{X}^{\dagger \sharp}, \mathcal{E}^{\dagger \sharp}\right) & \\
& \longrightarrow C^{\infty}\left(\mathcal{X}^{\dagger \sharp}, \mathcal{E}^{\dagger \sharp} \otimes p_{\mu}^{\dagger *} \Omega_{X^{\dagger}}^{1}\right) .
\end{aligned}
$$

The following lemma is clear from the definition.

Lemma 3.5. If $\lambda=\mu^{-1}$, then we have $\mathbb{D}^{\dagger \mu, f}=\mathbb{D}^{\lambda, f}$ as the operator $C^{\infty}(X, E) \longrightarrow C^{\infty}\left(X, E \otimes \Omega_{X}^{1}\right)$. Namely they give the same flat connection.

We have the morphism $\mathbf{C}_{\lambda}^{*} \longrightarrow \mathbf{C}_{\mu}^{*}$ by the correspondence $\mu=\lambda^{-1}$. It induces the $C^{\infty}$-morphism $\boldsymbol{\beta}_{X}: \mathcal{X}^{\sharp} \longrightarrow \mathcal{X}^{\dagger}$. Although $\boldsymbol{\beta}_{X}$ is not holomorphic, it is holomorphic in the direction of $\mathbf{C}_{\lambda}^{*}$. The following lemma can be shown directly from the definitions.

Lemma 3.6. Under the identification of $\mathcal{X}^{\sharp}$ and $\mathcal{X}^{\dagger \sharp}$ by the morphism $\boldsymbol{\aleph}_{X}$ above, we have the relation $\mathbb{D}^{f}=\mathbb{D}^{\dagger, f}$.

\subsubsection{Another relation between $\mathcal{E}$ and $\mathcal{E}^{\dagger}$}

Let $\left(E, \bar{\partial}_{E}, h\right)$ be a holomorphic vector bundle with a hermitian metric. In general, a hermitian metric $h$ induces an anti-linear morphism $\psi$ of $E$ to the dual $E^{\vee}$. The morphism $\psi$ gives an anti-holomorphic isomorphism of $E$ and $E^{\vee}$.

Let $\boldsymbol{v}=\left(v_{1}, \ldots, v_{n}\right)$ be a holomorphic frame of $E$. We have the dual frame $\boldsymbol{v}^{\vee}$ of $E^{\vee}$. Then we put $\boldsymbol{v}^{\dagger}=\psi^{-1}\left(\boldsymbol{v}^{\vee}\right)$. Namely we put as follows: we have the matrix $\left(b_{i, j}\right)=\overline{H(h, \boldsymbol{v})}^{-1}$, and frame $\boldsymbol{v}^{\dagger}=\left(v_{1}^{\dagger}, \ldots, v_{n}^{\dagger}\right)$ defined by the relation $v_{j}^{\dagger}:=\sum b_{i, j} \cdot v_{i}$, that is, $\boldsymbol{v}^{\dagger}=\boldsymbol{v} \cdot \overline{H(h, \boldsymbol{v})}^{-1}$. Then $\boldsymbol{v}^{\dagger}$ is an anti-holomorphic frame of $E$, in other words, $\boldsymbol{v}^{\dagger}$ is a holomorphic frame of $\left(E, \partial_{E}\right)$ over $X^{\dagger}$. 
Let $\left(E, \bar{\partial}_{E}, \theta, h\right)$ be a harmonic bundle over $X$. Then we have the deformed holomorphic bundle $\mathcal{E}^{\lambda}$ over $\mathcal{X}^{\lambda}$, whose holomorphic structure is given by $\bar{\partial}_{E}+\lambda \cdot \theta^{\dagger}$. The anti-holomorphic structure is given by $\partial_{E}-\bar{\lambda} \cdot \theta$. Thus $\boldsymbol{v}^{\dagger}$ gives a holomorphic frame of $\mathcal{E}^{\dagger(-\bar{\lambda})}$ over $\mathcal{X}^{\dagger(-\bar{\lambda})}$. The following lemma can be checked by a direct calculation.

Lemma 3.7. When we have the relation $\mathbb{D}^{\lambda} \boldsymbol{v}=\boldsymbol{v} \cdot \mathcal{A}$, then we have the relation $\mathbb{D}^{\dagger(-\bar{\lambda})} \boldsymbol{v}^{\dagger}=\boldsymbol{v}^{\dagger} \cdot{ }^{t} \overline{\mathcal{A}}$.

Proof. It follows from the following equality:

$$
\begin{aligned}
\left(\mathbb{D}^{\lambda} v_{i}, v_{j}^{\dagger}\right)_{h} & =\left(\left(\lambda \cdot \partial_{E}+\theta\right) v_{i}, v_{j}^{\dagger}\right)_{h} \\
& =\left(v_{i},\left(-\bar{\lambda} \partial_{E}+\theta^{\dagger}\right) v_{j}^{\dagger}\right)_{h}=\left(v_{i}, \mathbb{D}^{\dagger(-\bar{\lambda})} v_{j}\right)_{h} .
\end{aligned}
$$

q.e.d.

We denote the metric connection of $\left(\mathcal{E}, d^{\prime \prime}, h\right)$ by $d^{\prime \prime}+d^{\prime}$. Then we have the holomorphic vector bundle $\left(\mathcal{E}, d^{\prime}\right)$ over $\mathbf{C}_{\lambda}^{\dagger} \times X^{\dagger}$. We have a holomorphic map $F: \mathbf{C}_{\mu} \longrightarrow \mathbf{C}_{\lambda}^{\dagger}$ defined by $\bar{\lambda}=-\mu$. Then we have the naturally defined holomorphic map $F: \mathcal{X}^{\dagger}=\mathbf{C}_{\mu} \times X^{\dagger} \longrightarrow \mathbf{C}_{\lambda}^{\dagger} \times X^{\dagger}$.

Lemma 3.8. The holomorphic bundle $F^{-1}\left(\mathcal{E}, d^{\prime}\right)$ is same as $\left(\mathcal{E}^{\dagger}, d^{\prime \prime}\right)$. We also have $F^{-1} \mathbb{D}=\mathbb{D}^{\dagger}$.

Proof. It can be checked by direct calculations.

q.e.d.

Let $\boldsymbol{v}$ be a (not-necessarily holomorphic) frame of $\mathcal{E}$. Then $\boldsymbol{v}^{\dagger}$ can naturally be regarded as a frame of $\mathcal{E}^{\dagger}$ in the sense of Lemma 3.8.

The following lemmas can be checked by direct calculations.

Lemma 3.9. Let $\boldsymbol{v}_{i}(i=1,2)$ be frames of $\mathcal{E}$ related by the matrices $B$, that is, $\boldsymbol{v}_{1}=\boldsymbol{v}_{2} \cdot B$. Then we have the relation

$$
\boldsymbol{v}_{1}^{\dagger}=\boldsymbol{v}_{2}^{\dagger} \cdot F^{*}\left({ }^{t} \bar{B}^{-1}\right) \text {. }
$$

Here $F$ denotes the above morphism $\mathbf{C}_{\mu} \times X^{\dagger} \longrightarrow \mathbf{C}_{\lambda}^{\dagger} \times X^{\dagger}$ given by $\bar{\lambda}=-\mu$.

Lemma 3.10. Let $\boldsymbol{v}$ be a holomorphic frame of $\mathcal{E}$ and $\mathcal{A}$ be a $\lambda$ connection form of $\mathbb{D}$ with respect to $\boldsymbol{v}$. Then $F^{*}\left({ }^{t} \overline{\mathcal{A}}\right)$ is the $\mu$-connection form of $\mathbb{D}^{\dagger}$.

\subsubsection{Functoriality}

Let $\left(E, \bar{\partial}_{E}, \theta, h\right)$ be a harmonic bundle over $X$. We have various kinds of functorial constructions. We recall some of them to fix our notation. 
Dual. We denote the dual bundle of $E$ by $E^{\vee}$. The metric $h$ induces the naturally defined metric on $E^{\vee}$, which we denote by $h^{\vee}$. The Higgs field $\theta$ naturally induces the holomorphic section $\theta^{\vee}=-\theta$ of End $\left(E^{\vee}\right) \otimes \Omega_{X}^{1,0} \simeq \operatorname{End}(E) \otimes \Omega_{X}^{1,0}$. Then the tuple $\left(E^{\vee}, \bar{\partial}_{E^{\vee}}, \theta^{\vee}, h^{\vee}\right)$ gives a harmonic bundle.

Let $(\mathcal{E}, \mathbb{D})$ be a $\lambda$-connection. We have the naturally induced $\lambda$ connection $\mathbb{D}$ on $\mathcal{E}^{\vee}$. Let $f$ be a holomorphic section of $\mathcal{E}^{\vee}$ and $g$ be a holomorphic section of $\mathcal{E}$. We denote the natural pairing by $(\cdot, \cdot)$. We have the obvious $\lambda$-connection $\mathbb{D}$ of $\mathcal{O}_{\mathcal{X}}$. Then $\mathbb{D}^{\vee}$ is determined by the following relation:

$$
\mathbb{D}(f, g)=\left(\mathbb{D}^{\vee} f, g\right)+\left(f, \mathbb{D}^{\vee} g\right) .
$$

It is easy to see that the deformed holomorphic bundle with $\lambda$-connection of $\left(E^{\vee}, \bar{\partial}_{E^{\vee}}, \theta^{\vee}, h^{\vee}\right)$ is naturally isomorphic to $\left(\mathcal{E}^{\vee}, \mathbb{D}^{\vee}\right)$.

Similarly the conjugate deformed holomorphic bundle with $\mu$-connection of $\left(E^{\vee}, \bar{\partial}_{E^{\vee}}, \theta^{\vee}, h^{\vee}\right)$ is naturally isomorphic to the dual of $\left(\mathcal{E}^{\dagger}, \mathbb{D}^{\dagger}\right)$.

Tensor product. Let $\left(E_{i}, \bar{\partial}_{E_{i}}, \theta_{i}, h_{i}\right)(i=1,2)$ be harmonic bundles over $X$. We have the tensor product $E_{1} \otimes E_{2}$. We have the naturally defined metric $\widetilde{h}:=h_{1} \otimes h_{2}$ and the Higgs field $\widetilde{\theta}:=\theta_{1} \otimes \mathrm{id}_{E_{2}}+\mathrm{id}_{E_{1}} \otimes \theta_{2}$. Here $\mathrm{id}_{E}$ denotes the identity of $E$. The tuple $\left(E_{1} \otimes E_{2}, \widetilde{\theta}, \widetilde{h}\right)$ gives a harmonic bundle over $X$. It is denoted by $\left(E_{1}, \theta_{1}, h_{1}\right) \otimes\left(E_{2}, \theta_{2}, h_{2}\right)$.

It is clear that the deformed holomorphic bundle with $\lambda$-connection of $\left(E_{1}, \theta_{1}, h_{1}\right) \otimes\left(E_{2}, \theta_{2}, h_{2}\right)$ is naturally isomorphic to the tensor product $\left(\mathcal{E}_{1}, \mathbb{D}_{1}\right) \otimes\left(\mathcal{E}_{2}, \mathbb{D}_{2}\right)$. Here $\left(\mathcal{E}_{i}, \mathbb{D}_{i}\right)$ denotes the deformed holomorphic bundle of $\left(E_{i}, \theta_{i}, h_{i}\right)$. We have a similar relation for the conjugate deformed holomorphic bundles with $\mu$-connections.

Direct summand. Assume that $\left(E_{1}, \theta_{1}, h_{1}\right) \oplus\left(E_{2}, \theta_{2}, h_{2}\right)$ are harmonic bundles over $X$. Then it is easy to see that the direct summands $\left(E_{i}, \theta_{i}, h_{i}\right)$ are also harmonic bundles. We have the obvious relations for the deformed holomorphic bundles and the conjugate deformed holomorphic bundles.

The invariant part with respect to the group action. Let $\left(E, \bar{\partial}_{E}, \theta, h\right)$ be a harmonic bundle over $X$. Let $G$ be a finite group acting on the vector bundle $E$. We assume that the action of $G$ on $X$ is trivial, and preserves $\theta$ and the metric. We have the decomposition of $E$ by the irreducible representations of $G$. The direct summands are naturally harmonic bundles. Such decomposition is compatible with the 
construction of the deformed holomorphic bundles and the conjugate deformed holomorphic bundles.

Symmetric products and exterior products. We have the symmetric product and the exterior product of the harmonic bundle $\left(E, \bar{\partial}_{E}, \theta, h\right)$. They are characterized as the direct summands of the tensor products.

We denote the symmetric product by $\operatorname{Sym}^{l}\left(E, \bar{\partial}_{E}, \theta, h\right)=\left(\operatorname{Sym}^{l}(E)\right.$, $\left.\theta^{\operatorname{sym} l}, h^{\operatorname{Sym} l}\right)$. The deformed holomorphic bundle is denoted by $\left(\operatorname{Sym}^{l} \mathcal{E}\right.$, $\left.\mathbb{D}^{\text {sym } l}, h^{\operatorname{sym} l}\right)$.

We denote the exterior product by $\bigwedge^{l}\left(E, \bar{\partial}_{E}, \theta, h\right)=\left(\bigwedge^{l}(E), \theta^{\wedge l}, h^{\wedge l}\right)$. The deformed holomorphic bundle is denoted by $\left(\bigwedge^{l} \mathcal{E}, \mathbb{D}^{\wedge l}, h^{\wedge l}\right)$.

Determinant. As a special case of the exterior product, we have the determinant line bundle $\operatorname{det}(E)$. The metric $h$ naturally induces the metric of $\operatorname{det}(E)$, which we denote by $\operatorname{det}(h)$. We have the natural isomorphism End $(\operatorname{det}(E), \operatorname{det}(E)) \simeq \mathcal{O}_{X}$, and the trace morphism $\operatorname{tr}$ : End $(E, E) \longrightarrow \mathcal{O}_{X}$. The Higgs field $\theta$ naturally induces the Higgs field $\operatorname{tr}(\theta) \in \Gamma\left(X, \Omega^{1,0}\right)$. The tuple $\left(\operatorname{det}(E), \bar{\partial}_{\operatorname{det}(E)}, \operatorname{tr}(\theta), \operatorname{det}(h)\right)$ gives a harmonic bundle. The deformed holomorphic bundle is denoted by $(\operatorname{det}(\mathcal{E}), \operatorname{tr}(\mathbb{D}), \operatorname{det}(h))$.

Hom. Let $\left(E_{i}, \theta_{i}, h_{i}\right)(i=1,2)$ be harmonic bundles. Then we have

$$
\left(\operatorname{Hom}\left(E_{1}, E_{2}\right), \theta_{1}^{\vee}+\theta_{2}, h_{1}^{\vee} \otimes h_{2}\right) \simeq\left(E_{1}^{\vee}, \theta_{1}^{\vee}, h_{1}^{\vee}\right) \otimes\left(E_{2}, \theta_{2}, h_{2}\right) .
$$

We have the obvious relations for the deformed holomorphic bundles with $\lambda$-connections.

Pull back. Let $f: Y \longrightarrow X$ be a morphism of complex manifolds. Let $\left(E, \bar{\partial}_{E}, \theta, h\right)$ be a harmonic bundle over $X$. Then we have the pull back $\left(f^{*} E, f^{*} \theta, f^{*} h\right)$, which gives a harmonic bundle over $Y$. The deformed holomorphic bundle with $\lambda$-connection of $f^{*}(E, \theta, h)$ is naturally isomorphic to the pull back of $(\mathcal{E}, \mathbb{D})$.

\subsection{Model bundles}

In this subsection, we recall some examples of harmonic bundles over the punctured disc $\Delta^{*}$.

\subsubsection{Line bundles $L(a)$}

We put $L=\mathcal{O}_{\Delta^{*}} \cdot e$. For any real number $a \in \mathbf{R}$, we put $h_{a}(e, e)=|z|^{2 a}$. Then $h_{a}$ gives a hermitian metric of $L$. The anti-holomorphic structure 
$\partial_{L}$ is determined by

$$
\partial_{L}(e)=e \cdot a \frac{d z}{z}
$$

Then $\bar{\partial}_{L}+\partial_{L}$ gives a flat connection of $L$.

We have the trivial Higgs field $\theta=0$ of $L$, and the conjugate $\theta^{\dagger}=0$. Then the tuple $L(a)=\left(L, h_{a}, \theta\right)$ gives a harmonic bundle.

Consider the deformed holomorphic bundle $(\mathcal{L}(a), \mathbb{D})$ over $\mathbf{C}_{\lambda} \times \Delta^{*}$. The holomorphic structure $d^{\prime \prime}$ is $\bar{\partial}_{L}+\bar{\partial}_{\lambda}$. Thus the natural $C^{\infty}$-frame $p_{\lambda}^{-1}(e)$ is holomorphic. The $\lambda$-connection $\mathbb{D}$ is $\bar{\partial}_{L}+\lambda \cdot \partial_{L}$. Thus we have the following relation:

$$
\mathbb{D}(e)=e \cdot\left(\lambda a \frac{d z}{z}\right)
$$

The associated family of the flat connections is as follows:

$$
\mathbb{D}^{f}(e)=e \cdot\left(a \frac{d z}{z}\right)
$$

The parabolic structure and the eigenvalue of the residue. We have the natural prolongment of $\mathcal{L}(a)$ over $\mathbf{C}_{\lambda} \times \Delta^{*}$ to the holomorphic line bundle $\mathcal{L}(a)_{a}$ over $\mathbf{C}_{\lambda} \times \Delta$ by using the frame $v$ above. Note that this prolongment is same as the prolongment by increasing order $a$ (See Subsection 4.1). In fact, the norm $|v|$ is $|z|^{a}$. The $\lambda$-connection $\mathbb{D}$ is of log type on $\mathcal{L}(a)_{a}$. Namely, for a holomorphic section $f$ of $\mathcal{L}(a)_{a}$, $\mathbb{D}(f)$ is a holomorphic section of $\mathcal{L}(a)_{a} \otimes p_{\lambda}^{-1} \Omega^{1,0}(\log (O))$. The residue of $\mathbb{D}$ is $\lambda \cdot a \in \Gamma\left(\mathbf{C}_{\lambda}\right.$, End $\left.\left(\mathcal{L}(a)_{a \mid \mathbf{C}_{\lambda} \times O}\right)\right) \simeq \Gamma\left(\mathbf{C}_{\lambda}, \mathcal{O}\right)$.

We have the conjugate $\left(\mathcal{L}(a)^{\dagger}, \mathbb{D}^{\dagger}\right)$. The holomorphic structure is given by $\partial_{L}+\bar{\partial}_{\mu}$. The section $e^{\dagger}=|z|^{-2 a} \cdot e$ gives a holomorphic frame. The $\mu$-connection is $\mathbb{D}^{\dagger}=\partial_{L}+\mu \cdot \bar{\partial}_{L}$, and we have the relation:

$$
\mathbb{D}^{\dagger} e^{\dagger}=e^{\dagger} \cdot \mu \cdot(-a) \frac{d \bar{z}}{\bar{z}}
$$

The associated family of the flat connections are as follows:

$$
\mathbb{D}^{\dagger, f} e^{\dagger}=e^{\dagger} \cdot(-a) \frac{d \bar{z}}{\bar{z}}
$$




\subsubsection{Line bundles $L(\alpha)$}

We put $L=\mathcal{O} \cdot e$ and $h_{0}(e, e)=1$. Then we have $\partial_{L}(e)=0$. For any complex number $\alpha \in \mathbf{C}$, we have the Higgs field $\theta=\alpha \cdot d z / z$. The conjugate $\theta^{\dagger}$ is $\bar{\alpha} \cdot d \bar{z} / \bar{z}$. We have the following relations:

$$
R\left(\partial_{L}+\bar{\partial}_{L}\right)=0, \quad \theta \wedge \theta^{\dagger}+\theta^{\dagger} \wedge \theta=|\alpha|^{2}(d z \cdot d \bar{z}+d \bar{z} \cdot d z)=0 .
$$

Thus $L(\alpha)=\left(L, h_{0}, \theta\right)$ is a harmonic bundle.

We have the deformed holomorphic bundle $(\mathcal{L}(\alpha), \mathbb{D})$. The holomorphic structure is given by the operator $d^{\prime \prime}=\bar{\partial}_{L}+\lambda \bar{\alpha} \cdot d \bar{z} / \bar{z}+\bar{\partial}_{\lambda}$. Thus we have the relation:

$$
d^{\prime \prime}(e)=e \cdot(\lambda \bar{\alpha}) \frac{d \bar{z}}{\bar{z}}
$$

We put $v=\exp \left(-\lambda \bar{\alpha} \log |z|^{2}\right) \cdot e$. Then $v$ gives a holomorphic frame of $\mathcal{L}(\alpha)$. We have the equality:

$$
h(v, v)=\exp \left(-(\lambda \bar{\alpha}+\bar{\lambda} \alpha) \log |z|^{2}\right)=|z|^{-4 \operatorname{Re}(\lambda \bar{\alpha})} .
$$

The $\lambda$-connection $\mathbb{D}$ is as follows:

$$
\mathbb{D}(v)=\left(\lambda \partial_{L}+\theta\right) v=(\lambda(-\lambda \bar{\alpha})+\alpha) \cdot v \cdot \frac{d z}{z}=v \cdot\left(-\lambda^{2} \bar{\alpha}+\alpha\right) \frac{d z}{z} .
$$

The associated family of the flat connection is as follows:

$$
\mathbb{D}^{f} v=v \cdot\left(-\lambda \bar{\alpha}+\lambda^{-1} \alpha\right) \frac{d z}{z} .
$$

The parabolic structure and the eigenvalues of the residues. Fix a $\lambda \in \mathbf{C}_{\lambda}$. We have the natural prolongment of $\mathcal{L}(\alpha)^{\lambda}$ over $\Delta^{*}$ to the line bundle $\mathcal{L}(\alpha)_{-2 \operatorname{Re}(\lambda \bar{\alpha})}^{\lambda}$ by using the holomorphic $v$ above. Note that the prolongment is same as the prolongment by the increasing order $-2 \operatorname{Re}(\lambda \bar{\alpha})$. (See Subsection 4.1). In fact, the norm $|v|$ is $|z|^{-2 \operatorname{Re}(\lambda \bar{\alpha})}$. The $\lambda$-connection $\mathbb{D}$ is of $\log$ type on the $\mathcal{L}(\alpha)_{-2 \operatorname{Re}(\lambda \bar{\alpha})}$. The residue is $\left(-\lambda^{2} \bar{\alpha}+\alpha\right) \in \operatorname{End}\left(\mathcal{L}(\alpha)_{-2 \operatorname{Re}(\lambda \bar{\alpha}) \mid(\lambda, O)}\right) \simeq \mathbf{C}$.

\subsubsection{Line bundles $\mathcal{L}(a, \alpha)$}

As is already seen, we have the harmonic bundles $\mathcal{L}(a)$ and $\mathcal{L}(\alpha)$ for $a \in \mathbf{R}$ and $\alpha \in \mathbf{C}$. The tensor product $\mathcal{L}(a, \alpha):=\mathcal{L}(a) \otimes \mathcal{L}(\alpha)$ is also a harmonic bundle. It is a tuple $\left(L, h_{a}, \alpha \cdot d z / z\right)$. We denote the 
deformed holomorphic bundle by $\mathcal{L}(a, \alpha)$. We have the natural frame $v=\exp \left(-\lambda \bar{\alpha} \log |z|^{2}\right) \cdot e$ of $\mathcal{L}(a, \alpha)$. We have the following:

$$
\begin{gathered}
h_{a}(v, v)=|z|^{2(a-2 \operatorname{Re}(\lambda \bar{\alpha}))}, \quad \mathbb{D}(v)=v \cdot\left(-\lambda^{2} \bar{\alpha}+\alpha+\lambda a\right) \frac{d z}{z}, \\
\mathbb{D}^{f}(v)=v \cdot\left(-\lambda \bar{\alpha}+\lambda^{-1} \alpha+a\right) \frac{d z}{z} .
\end{gathered}
$$

Fix a complex number $\lambda$. We have the prolongment of $\mathcal{L}(\alpha, a)^{\lambda}$ over $\Delta^{*}$ to the line bundle $\left(\mathcal{L}(\alpha, a)^{\lambda}\right)_{a-2 \operatorname{Re}(\lambda \bar{\alpha})}$ over $\Delta$. This is same as the prolongment by the increasing order $a-2 \operatorname{Re}(\lambda \bar{\alpha})$. (See Subsection 4.1). The $\lambda$-connection $\mathbb{D}^{\lambda}$ is of log type. The residue is $-\lambda^{2} \bar{\alpha}+\alpha+\lambda a$.

Lemma 3.11. Take an arbitrary pair $(A, B) \in \mathbf{R} \times \mathbf{C}$. Fix a complex number $\lambda$. We can take a pair $(a, \alpha) \in \mathbf{R} \times \mathbf{C}$ such that the parabolic structure of $\mathcal{L}(a, \alpha)_{a-2 \operatorname{Re}(\lambda \bar{\alpha})}^{\lambda}$ is $A$, and that the residue of $\mathbb{D}^{\lambda}$ is same as $B$. In fact, we only have to put as follows:

$$
a=\frac{\left(1-|\lambda|^{2}\right) \cdot A+2 \operatorname{Re}(\bar{\lambda} \cdot B)}{1+|\lambda|^{2}}, \quad \alpha=\frac{B-\lambda \cdot A}{1+|\lambda|^{2}}
$$

Proof. We only have to check that $A=a-2 \operatorname{Re}(\lambda \bar{\alpha})$ and $B=$ $-\lambda^{2} \bar{\alpha}+\alpha+\lambda a$. It can be checked by a direct calculation. $\quad$ q.e.d.

\subsubsection{Mod $(l, a, C)$}

We put $V_{2}=\mathbf{C} \cdot e_{1} \oplus \mathbf{C} \cdot e_{-1}$. We have the nilpotent map $N_{2}$ defined by $N_{2}\left(e_{1}\right)=e_{-1}$ and $N_{2}\left(e_{-1}\right)=0$. We have the holomorphic vector bundle $E_{2}:=V_{2} \otimes \mathcal{O}_{\Delta^{*}}$, which have the natural frame $\boldsymbol{e}=\left(e_{1}, e_{-1}\right)$. We have the Higgs field $\theta_{2}=N_{2} \cdot d z / z$. We have the following relation:

$$
\theta_{2}\left(e_{1}, e_{-1}\right)=\left(e_{-1}, 0\right)=\left(e_{1}, e_{-1}\right)\left(\begin{array}{ll}
0 & 0 \\
1 & 0
\end{array}\right) \frac{d z}{z}
$$

We put $y=-\log |z|^{2}$. We take a metric $h_{2}$ as follows:

$$
h_{2}\left(e_{-1}, e_{-1}\right)=y^{-1}, \quad h_{2}\left(e_{-1}, e_{1}\right)=0, \quad h_{2}\left(e_{1}, e_{1}\right)=y .
$$

The conjugate $\theta_{2}^{\dagger}$ of $\theta_{2}$ with respect to the metric $h_{2}$ is as follows:

$$
\theta_{2}^{\dagger}\left(e_{1}, e_{-1}\right)=\left(e_{1}, e_{-1}\right) \cdot\left(\begin{array}{cc}
0 & 1 \\
0 & 0
\end{array}\right) \frac{d \bar{z}}{\bar{z} \cdot y^{2}}
$$


Lemma 3.12. A tuple $\operatorname{Mod}(2)=\left(E_{2}, \bar{\partial}_{E_{2}}, h_{2}, \theta_{2}\right)$ gives a harmonic bundle.

Proof. We denote the hermitian matrix $H\left(h_{2}, \boldsymbol{e}\right)$ by $H$. The matrix $H$ and and the connection form $H^{-1} \partial H$ is as follows:

$$
H=\left(\begin{array}{cc}
y & 0 \\
0 & y^{-1}
\end{array}\right), \quad H^{-1} \partial H=\left(\begin{array}{cc}
-y & 0 \\
0 & y^{-1}
\end{array}\right) \frac{d z}{z \cdot y} .
$$

The curvature $R\left(\partial_{E_{2}}+\bar{\partial}_{E_{2}}\right)=\bar{\partial}\left(H^{-1} \partial H\right)$ is as follows:

$$
\bar{\partial}\left(H^{-1} \partial H\right)=\left(\begin{array}{cc}
1 & 0 \\
0 & -1
\end{array}\right) \frac{d z \cdot d \bar{z}}{y^{2} \cdot|z|^{2}} .
$$

On the other hand, we have the following:

$$
\theta_{2} \cdot \theta_{2}^{\dagger}+\theta_{2}^{\dagger} \cdot \theta_{2}=\left(\left(\begin{array}{cc}
0 & 0 \\
0 & 1
\end{array}\right)+\left(\begin{array}{cc}
-1 & 0 \\
0 & 0
\end{array}\right)\right) \frac{d z \cdot d \bar{z}}{|z|^{2} \cdot y^{2}}
$$

Thus we obtain the equality $R\left(\bar{\partial}_{E_{2}}+\partial_{E_{2}}\right)+\left[\theta_{2}, \theta_{2}^{\dagger}\right]=0$. The other relations $\partial_{E_{2}}\left(\theta_{2}\right)=0$ and $\bar{\partial}_{E_{2}}\left(\theta_{2}^{\dagger}\right)$ are trivial in this case, because we have $\Omega_{\Delta^{*}}^{2,0}=\Omega_{\Delta^{*}}^{0,2}=0$.

q.e.d.

Thus we obtain the harmonic bundle Mod (2). Note that we have the natural frame $\boldsymbol{e}=\left(e_{1}, e_{-1}\right)$.

On the vector space $\bigotimes^{l-1} V_{2}(l \geq 1)$, we have the nilpotent map

$$
N_{l}:=\sum_{a=0}^{l-2} \overbrace{1 \otimes \cdots \otimes 1}^{a} \otimes N_{2} \otimes 1 \otimes \cdots \otimes 1 .
$$

We have the action of $(l-1)$-th symmetric group $\mathfrak{S}_{l-1}$ on the $(l-1)$-th tensor product $V_{2}^{\otimes(l-1)}$ defined by the transposition of the components. The $l$-dimensional vector space $V_{l}:=\operatorname{Sym}^{l-1}\left(V_{2}\right)$ is characterized as the invariant part of the action. Since the nilpotent map $N_{l}$ commutes with the action of $\mathfrak{S}_{l-1}$, the map $N_{l}$ preserves the subspace $V_{l}$. We denote the restriction of $N_{l}$ to $V_{l}$ also by $N_{l}$.

We have the harmonic bundle $\left(E_{2}^{\otimes(l-1)}, \theta_{l}, h_{l}\right)$, where we put $\theta_{l}=$ $N_{l} d z / z$ and $h_{l}=\otimes^{l-1} h_{2}$. We have the natural inclusion $E_{l}:=V_{l} \otimes \mathcal{O} \subset$ $E_{2}^{\otimes(l-1)}$, which is the invariant part of the $\mathfrak{S}_{l-1}$-action. The action of $\mathfrak{S}_{l-1}$ preserves $\bar{\partial}_{E_{2}^{\otimes(l-1)}}, \theta_{l}$ and $h_{l}$. Thus the action preserves $\partial_{E_{2}^{\otimes(l-1)}}$, 
and $\theta_{l}^{\dagger}$. Hence $\partial_{E_{2}^{\otimes(l-1)}}, \theta_{l}$ and $\theta_{l}^{\dagger}$ preserves the subbundle $E_{l}$. The adjoint of $\left.\theta_{l}\right|_{E_{l}}$ with respect to the metric $\left.h_{l}\right|_{E_{l}}$ is same as the restriction $\left.\theta_{l}^{\dagger}\right|_{E_{l}}$. We denote the restrictions of $\theta_{l}, \theta_{l}^{\dagger}$ and $h_{l}$ to $E_{l}$ by the same notation. From the consideration above, it is easy to see the following.

Lemma 3.13. The tuple $\operatorname{Mod}(l)=\left(E_{l}, \theta_{l}, h_{l}\right)$ is a harmonic bundle.

Thus we obtain the harmonic bundle $\operatorname{Mod}(l)$. Note that we have the characterization of $\operatorname{Mod}(l)$ as the $\mathfrak{S}_{l-1}$-fixed part of $\operatorname{Mod}(2)^{\otimes(l-1)}$. Note also that we have the natural frame $\boldsymbol{e}=\left(e^{p, q} \mid p+q=l-1\right)$ defined as follows:

$$
e^{p, q}:=e_{1}^{p} \cdot e_{-1}^{q}
$$

Here the product is the symmetric product. Clearly $\boldsymbol{e}$ is an orthogonal holomorphic frame of $E_{l}$.

Take a positive number $a>0$ and a complex number $C \in \mathbf{C}$ such that $|C| \leq 1$. Then the following is easy.

Lemma 3.14. The tuple $\operatorname{Mod}(l, a, C)=\left(E_{l}, \theta_{l}, a \cdot h_{l}(C \cdot z)\right)$ gives a harmonic bundle for any $a>0$ and $0<|C| \leq 1$.

Proof. We have no contribution of the positive constant $a$ to the condition for the harmonic bundles. For any $C$ such that $0<|C| \leq 1$, we have the morphism $f_{c}: \Delta^{*} \longrightarrow \Delta^{*}$ defined by $z \longmapsto C \cdot z$. Then we have the isomorphism between $\operatorname{Mod}(l, a, C)$ and $f_{c}^{*} \operatorname{Mod}(l, a, 1)$ by using the natural frame $\boldsymbol{e}$. Thus $\operatorname{Mod}(l, a, C)$ is also harmonic. q.e.d.

We have the prolongment of $E_{l}$ over $\Delta^{*}$ to $\left(E_{l}\right)_{0}$ over $\Delta$, by using the frame $\boldsymbol{e}$. Note that it is same as the prolongment by the increasing order 0. (See Subsection 4.1). The $\theta_{l}$ is of log type. The residue $\left(\left(E_{l}\right)_{0 \mid O}, \operatorname{Res}\left(\theta_{l}\right)\right)$ is naturally isomorphic to $\left(V_{l}, N_{l}\right)$.

Let $V$ be a finite dimensional vector bundle, and $N$ be a nilpotent map on $V$. We can take an isomorphism $(V, N) \simeq \bigoplus_{i}\left(V_{l_{i}}, N_{l_{i}}\right)$. Thus we have the following:

Lemma 3.15. For any pair $(V, N)$ as above, we have a harmonic bundle $\bigoplus_{i} \operatorname{Mod}\left(l_{i}, a_{i}, C_{i}\right)$ such that the residue is isomorphic to $(V, N)$.

Notation. Although we do not have a canonical choice of such harmonic bundle, we often denote such harmonic bundle by $E(V, N)$ for simplicity. 


\subsubsection{The deformed holomorphic bundle of $\operatorname{Mod}(2, a, C)$}

First we see the deformed holomorphic bundle $\operatorname{Mod}(2, a, C)$ of $\operatorname{Mod}(2$, $a, C)$. We put $y=-\log |z|^{2}$ and $c=-\log |C|^{2} \geq 0$. We have the natural $C^{\infty}$-section $p_{\lambda}^{-1} e_{i}(i=1,-1)$ of $\operatorname{Mod}(2, a, C)$. For simplicity, we denote $p_{\lambda}^{-1} e_{i}$ by $e_{i}$. We put as follows:

$$
v^{1,0}=e_{1}, \quad v^{0,1}=e_{-1}-\frac{\lambda}{y+c} \cdot e_{1} .
$$

Then it can be directly checked that $v^{1,0}$ and $v^{0,1}$ are holomorphic. Thus $\boldsymbol{v}=\left(v^{1,0}, v^{0,1}\right)$ gives a holomorphic frame of $\operatorname{Mod}(2, a, C)$. The $\lambda$-connection $\mathbb{D}$ is as follows:

$$
\begin{array}{r}
\mathbb{D}\left(v^{1,0}, v^{0,1}\right)=\left(v^{0,1} \cdot d z / z, 0\right)=\left(v^{1,0}, v^{0,1}\right) \cdot\left(\begin{array}{ll}
0 & 0 \\
1 & 0
\end{array}\right) \frac{d z}{z} \\
\text { i.e., } \mathbb{D} \boldsymbol{v}=\boldsymbol{v} \cdot\left(\begin{array}{ll}
0 & 0 \\
1 & 0
\end{array}\right) \frac{d z}{z} .
\end{array}
$$

The associated family of the flat connections is as follows:

$$
\mathbb{D}^{f} \boldsymbol{v}=\boldsymbol{v} \cdot\left(\begin{array}{cc}
0 & 0 \\
\lambda^{-1} & 0
\end{array}\right) \frac{d z}{z}
$$

To see the conjugate $\mathcal{M o d}(2, a, C)^{\dagger}$, the frame $\boldsymbol{v}^{\dagger}=\left(v^{\dagger 1,0}, v^{\dagger, 0,1}\right)$ is given by the following formula:

$$
\boldsymbol{v}^{\dagger}=\boldsymbol{v} \cdot{\overline{H\left(h_{2}, \boldsymbol{v}\right)}}^{-1}
$$

Then $\boldsymbol{v}^{\dagger}$ gives a holomorphic frame of $\operatorname{Mod}(2, a, C)^{\dagger}$, and we have the following relation:

$$
\mathbb{D}^{\dagger} \boldsymbol{v}^{\dagger}=\boldsymbol{v}^{\dagger} \cdot\left(\begin{array}{cc}
0 & 1 \\
0 & 0
\end{array}\right) \frac{d \bar{z}}{\bar{z}}, \quad \mathbb{D}^{\dagger f} \boldsymbol{v}^{\dagger}=\boldsymbol{v}^{\dagger} \cdot\left(\begin{array}{cc}
0 & \mu^{-1} \\
0 & 0
\end{array}\right) \frac{d \bar{z}}{\bar{z}} .
$$

We take a $C^{\infty}$-frame $\boldsymbol{v}^{\prime}=\left(v^{\prime 1,0}, v^{\prime 0,1}\right)$ given as follows:

$$
v^{\prime 1,0}=y^{-1 / 2} \cdot v^{1,0}, \quad v^{\prime 0,1}=y^{1 / 2} \cdot v^{0,1} .
$$

Consider the transformation matrices $B_{0}$ and $B_{0}^{\prime}$ defined as follows:

$$
\boldsymbol{v}=\boldsymbol{e} \cdot B_{0}=\boldsymbol{v}(0) \cdot B_{0}, \quad \boldsymbol{v}^{\prime}=\boldsymbol{v}^{\prime}(0) \cdot B_{0}^{\prime}
$$


Here $\boldsymbol{v}(0)$ denotes $p_{\lambda}^{-1}\left(\left.\boldsymbol{v}\right|_{\lambda=0}\right)$ precisely. Similar to $\boldsymbol{v}^{\prime}(0)$. Clearly $B_{0}$ and $B_{0}^{\prime}$ are elements of the space $C^{\infty}\left(\mathbf{C}_{\lambda} \times \Delta^{*}, M(r)\right)$. In fact, they are given as follows:

$$
\begin{aligned}
& B_{0}(\lambda, z)=\left(\begin{array}{cc}
1 & -\lambda \cdot(y+c)^{-1} \\
0 & 1
\end{array}\right), \\
& B_{0}^{\prime}(\lambda, z)=\left(\begin{array}{cc}
1 & -\lambda \cdot y \cdot(y+c)^{-1} \\
0 & 1
\end{array}\right) .
\end{aligned}
$$

Lemma 3.16. Let $B_{0}$ and $B_{0}^{\prime}$ be as above.

1. Let $R_{1}$ and $R_{2}$ be real numbers satisfying $0<R_{1}$ and $0<R_{2}<1$. The function $B_{0}(\lambda, z)$ is bounded over $\Delta_{\lambda}\left(R_{1}\right) \times \Delta_{z}^{*}\left(R_{2}\right)$. The boundedness is independent of $C$ with $|C| \leq 1$.

2. Let $R_{1}$ be a real number satisfying $R_{1}>0$. The function $B_{0}^{\prime}(\lambda, z)$ is bounded over $\Delta_{\lambda}\left(R_{1}\right) \times \Delta^{*}$. The boundedness is independent of $C$ with $|C| \leq 1$.

In particular, the $C^{\infty}$-frame $\boldsymbol{v}^{\prime}$ is adapted on the region.

Proof. The boundedness of the $B_{0}$ and $B_{0}^{\prime}$ are clear. It is easy to directly see that $\boldsymbol{v}^{\prime}(0)$ is adapted. Then the adaptedness of $\boldsymbol{v}^{\prime}$ follows from the boundedness of $B_{0}^{\prime}$.

q.e.d.

For the conjugate, the $C^{\infty}$-frame $\boldsymbol{v}^{\prime \dagger}=\left(v^{\prime \dagger, 1,0}, v^{\prime \dagger, 0,1}\right)$ is given as follows:

$$
v^{\dagger, 1,0}=y^{1 / 2} v^{\dagger 1,0}, \quad v^{\dagger, 0,1}=y^{-1 / 2} v^{\dagger 0,1} .
$$

Then we obtain the transformation matrices $B_{0}^{\dagger}$ and $B_{0}^{\prime \dagger}$ satisfying $\boldsymbol{v}^{\dagger}=$ $\boldsymbol{v}^{\dagger}(0) \cdot B_{0}^{\dagger}$ and $\boldsymbol{v}^{\prime \dagger}=\boldsymbol{v}^{\prime \dagger}(0) B_{0}^{\prime \dagger}$. The formula of $B_{0}^{\dagger}$ and $B_{0}^{\prime \dagger}$ are given as follows:

$$
\begin{aligned}
B_{0}^{\dagger}(\mu, \bar{z}) & =\left(\begin{array}{cc}
1 & 0 \\
-\mu \cdot(y+c)^{-1} & 1
\end{array}\right) \\
B_{0}^{\prime \dagger}(\mu, \bar{z}) & =\left(\begin{array}{cc}
1 & 0 \\
-\mu \cdot y \cdot(y+c)^{-1} & 1
\end{array}\right) .
\end{aligned}
$$

\subsubsection{The deformed holomorphic bundle of $\operatorname{Mod}(l+1, a, C)$}

We describe the deformed holomorphic bundle $\operatorname{Mod}(l+1, a, C)$ of $\operatorname{Mod}(l+1, a, C)$. We remark that the bundle $\operatorname{Mod}(l+1, a, C)$ is the 
$\mathfrak{S}_{l}$-invariant part of $\bigotimes^{l} \operatorname{Mod}(2, a, C)$. Thus the following holomorphic sections $\boldsymbol{v}=\left(v^{p, q} \mid p+q=l\right)$ of $\otimes^{l} \operatorname{Mod}(2, a, C)$ gives a holomorphic frame of $\operatorname{Mod}(l+1, a, C)$ :

$$
v^{p, q}:=\left(v^{1,0}\right)^{p} \cdot\left(v^{0,1}\right)^{q} .
$$

Here the product is the symmetric product. The $\lambda$-connection $\mathbb{D}$ is described as follows:

$$
\mathbb{D}\left(v^{p, q}\right)=p \cdot v^{p-1, q+1} \frac{d z}{z} .
$$

By using the trivialization $\boldsymbol{v}$, the $\operatorname{Mod}(l+1, a, C)$ is prolonged to the holomorphic vector bundle over $\mathbf{C}_{\lambda} \times \Delta$. It is same as the prolongment by the increasing order 0. (See Subsection 4.1). Thus the prolongment is denoted by $\operatorname{Mod}(l+1, a, C)_{0}$.

The $\lambda$-connection $\mathbb{D}$ is of $\log$ type on $\operatorname{Mod}(l+1, a, C)_{0}$. Thus we obtain the residue:

$$
\operatorname{Res}(\mathbb{D}) \in \Gamma\left(\mathbf{C}_{\lambda} \times\{O\}, \operatorname{End}\left(\operatorname{Mod}(l+1, a, C)_{0 \mid \mathbf{C}_{\lambda} \times\{O\}}\right)\right) .
$$

Lemma 3.17. The conjugacy class of $\operatorname{Res}(\mathbb{D})$ of the model bundle is independent of $\lambda$.

Proof. Clear from Equation (12).

q.e.d.

Corollary 3.1. Let $V$ be a finite dimensional vector space and $N$ be a nilpotent map on $V$. Let $E(V, N)$ be a harmonic bundle whose residue of the Higgs field is isomorphic to $(V, N)$. Then the residue of the $\lambda$-connection is also isomorphic to $(V, N)$.

We have the $C^{\infty}$-frame $\boldsymbol{v}^{\prime}=\left(v^{\prime p, q} \mid p+q=l\right)$ of $\operatorname{Mod}(l+1, a, C)$, defined as follows:

$$
v^{p, q}:=v^{p, q} \cdot y^{\frac{q-p}{2}} .
$$

We have the transformation matrices $B_{0}=\left(B_{0, i, p}\right)$ and $B_{0}^{\prime}=\left(B_{0, i, p}^{\prime}\right)$ satisfying the equalities, $\boldsymbol{v}=\boldsymbol{v}(0) \cdot B_{0}$ and $\boldsymbol{v}^{\prime}=\boldsymbol{v}^{\prime}(0) \cdot B_{0}^{\prime}$, or more precisely:

$$
\begin{aligned}
v^{p, l-p}(\lambda, z) & =\sum_{i} B_{0, i, p}(\lambda, z) \cdot v^{i, l-i}(0, z), \\
v^{\prime p, l-p}(\lambda, z) & =\sum_{i} B_{0, i, p}^{\prime}(\lambda, z) \cdot v^{\prime, i, l-i}(0, z) .
\end{aligned}
$$

They are elements of $C^{\infty}\left(\mathbf{C}_{\lambda} \times \Delta^{*}, M(r)\right)$. 
Lemma 3.18. We have the following equalities:

$$
B_{0, i, p}(\lambda, z)= \begin{cases}0, & (i<p) \\ c(l-p, i-p) \cdot \lambda^{i-p} \cdot(-y-c)^{-i+p}, & (i \geq p) .\end{cases}
$$

Here $c(l, p)$ denotes the constant given as the coefficient of $x^{p}$ in the polynomial $(1+x)^{l}$.

Similarly we have the following:

$$
\left(B_{0}\right)_{i, p}^{-1}(\lambda, z)= \begin{cases}0, & (i<p) \\ c(l-p, i-p) \cdot \lambda^{i-p} \cdot(y+c)^{-i+p}, & (i \geq p) .\end{cases}
$$

As a result, the matrices $B_{0}$ and $B_{0}^{-1}$ are bounded on the region $\Delta_{\lambda}\left(R_{1}\right) \times \Delta_{z}^{*}\left(R_{2}\right)$ for any $0<R_{1}$ and $0<R_{2}<1$. The boundedness is independent of the parameter $C \geq 0$.

Proof. We put $\gamma=-\lambda \cdot(y+c)^{-1}$. Then we have the following equalities:

$$
\begin{aligned}
v^{p, l-p}(\lambda, z) & =e_{1}^{p} \cdot\left(e_{-1}(z)+\gamma \cdot e_{1}(z)\right)^{l-p} \\
& =\sum_{j=0}^{l-p} e_{1}^{p} \cdot c(l-p, j) \cdot e_{-1}^{j}(z) \cdot e_{1}^{l-p-j}(z) \cdot \gamma^{l-p-j} \\
& =\sum_{i=p}^{l-p} c(l-p, i-p) \gamma^{i-p} \cdot e_{1}^{i}(z) \cdot e_{-1}^{l-i}(z) \\
& =\sum_{i=p}^{l} c(l-p, i-p) \cdot \gamma^{i-p} \cdot v^{i, l-i}(0, z) .
\end{aligned}
$$

Thus we are done.

q.e.d.

We have the following immediate corollary.

Corollary 3.2. We have the following equalities:

$$
B_{0, i, p}^{\prime}(\lambda, z)= \begin{cases}0, & (i<p), \\ c(l-p, i-p) \cdot \lambda^{i-p} \cdot(-y-c)^{-i+p} \cdot y^{i-p}, & (i \geq p) .\end{cases}
$$

We also have the following equalities:

$$
\left(B_{0}^{\prime-1}\right)_{i, p}(\lambda, z)= \begin{cases}0, & (i<p), \\ c(l-p, i-p) \cdot \lambda^{i-p} \cdot(y+c)^{-i+p} \cdot y^{i-p}, & (i \geq p) .\end{cases}
$$

In particular, $B_{0}^{\prime}(\lambda, z)$ and their inverses are bounded on the region $\Delta_{\lambda}\left(R_{1}\right) \times \Delta_{z}^{*}$ for any $0<R_{1}$. The boundedness is independent of the parameter $C \geq 0$. 
Proof. The formula is obtained from (14). The boundedness follows from the boundedness of $y^{-1}(y+c)$.

q.e.d.

\section{Corollary 3.3.}

1. We put $H_{l}:=H\left(h_{l}, \boldsymbol{v}\right)$. Then $H_{l}(\lambda, z)$ and $H_{l}(0, z)$ are mutually bounded over $\Delta_{\lambda}\left(R_{1}\right) \times \Delta_{z}^{*}\left(R_{2}\right)$ for any $0<R_{1}$ and $0<R_{2}<1$.

2. The $C^{\infty}$-frame $\boldsymbol{v}^{\prime}$ is adapted over $\Delta_{\lambda}\left(R_{1}\right) \times \Delta_{z}^{*}$ for any $R_{1}>0$.

Proof. The first claim follows from the boundedness of $B_{0}$ and $B_{0}^{-1}$. The adaptedness of $\boldsymbol{v}^{\prime}(0)$ is easy to see. Thus the adaptedness of $\boldsymbol{v}^{\prime}$ is obtained from the boundedness of $B_{0}^{\prime}$.

Otherwise, it is also easy to see that the adaptedness of $\boldsymbol{v}^{\prime}$ follows from the adaptedness of $\boldsymbol{v}^{\prime}$ in the case $l=2$.

q.e.d.

Corollary 3.4. When $|z| \rightarrow 0, B_{0}^{\prime}(\lambda, z)$ converge to $B_{0}^{\complement}$ :

$$
\begin{aligned}
B_{0, j, p}^{\odot}(\lambda) & = \begin{cases}0, & (i<p), \\
c(l-p, i-p) \cdot \lambda^{i-p} \cdot(-1)^{i-p}, & (i \geq p),\end{cases} \\
\left(B_{0}^{\odot-1}\right)_{j, p}(\lambda) & = \begin{cases}0, & (i<p), \\
c(l-p, i-p) \cdot \lambda^{i-p}, & (i \geq p),\end{cases}
\end{aligned}
$$

In particular, we have $\left(B_{0}^{\bigodot}\right)_{p, p}=\left(B_{0}^{\bigodot-1}\right)_{p, p}=1$ for any $0 \leq p \leq l$. We also have $\left(B_{0}^{\complement}\right)_{l, 0}=(-1)^{l} \cdot \lambda^{l}$ and $\left(B_{0}^{\complement-1}\right)_{l, 0}=\lambda^{l}$.

Proof. This is a direct corollary of Corollary 3.2.

q.e.d.

It will be convenient to replace the adaptedness for $\boldsymbol{v}^{\prime}$ in Corollary 3.5 with the adaptedness for some more flexible frame. We have the residues $\operatorname{Res}(\mathbb{D})$, which is a holomorphic section of End $(\operatorname{Mod}(l+$ $\left.1, a, C)_{0}\right)$ on $\mathbf{C}_{\lambda} \times\{O\}$. Since the conjugacy classes are independent of $\lambda$, the nilpotent maps $\operatorname{Res}(\mathbb{D})_{\mid(\lambda, O)}$ induces the weight filtration $W$ of $\left.\mathcal{M o d}(l+1, a, C)\right|_{\mathbf{C}_{\lambda} \times O}$ by vector subbundles. Take a holomorphic frame $\boldsymbol{w}$ of $\operatorname{Mod}(l+1, a, C)_{0}$, which is compatible with the filtration $W$ on $\mathbf{C}_{\lambda} \times\{O\}$. We put as follows:

$$
k\left(w_{i}\right):=\frac{1}{2} \operatorname{deg}^{W}\left(w_{i}\right) .
$$

We have the $C^{\infty}$-frame $\boldsymbol{w}^{\prime}=\left(w_{1}^{\prime}, \ldots, w_{l+1}^{\prime}\right)$ of $\operatorname{Mod}(l+1, a, C)$ over $\Delta^{*}$ defined as follows:

$$
w_{i}^{\prime}:=y^{-k\left(w_{i}\right)} \cdot w_{i}
$$

The following is an easy corollary of Corollary 3.3. 
Corollary 3.5. Let $\boldsymbol{w}^{\prime}$ be as above. Then $\boldsymbol{w}^{\prime}$ is adapted over $\Delta_{\lambda}(R) \times X$ for any $R>0$.

Proof. We only have to see that the transformation matrices of $\boldsymbol{w}^{\prime}$ and $\boldsymbol{v}^{\prime}$ and their inverses are bounded. The section $w_{i}$ is described by $\boldsymbol{v}$ as follows:

$$
w_{i}=\sum_{p} C_{p, i} \cdot v^{p, l-p} .
$$

Here $C_{p, i}$ are holomorphic on $\mathbf{C}_{\lambda} \times \Delta$. Since both of $\boldsymbol{w}$ and $\boldsymbol{v}$ are compatible with the filtration $W$, the following holds:

$$
\text { If } \operatorname{deg}^{W}\left(w_{i}\right)<\operatorname{deg}^{W}\left(v^{p, l-p}\right) \text {, then } C_{p, i}(\lambda, O)=0 .
$$

We have the following relation:

$$
w_{i}^{\prime}=\sum_{p} C_{p, i} \cdot y^{-k\left(w_{i}\right)+k\left(v^{p, l-p}\right)} \cdot v^{\prime p, l-p} .
$$

Then the function $C_{p, i} \cdot y^{-k\left(w_{i}\right)+k\left(v^{p, l-p}\right)}$ is bounded over $\Delta_{\lambda}(R) \times X$ for any $R>0$. Similarly the inverse of the transformation matrices are bounded.

q.e.d.

Similarly we can see the conjugate $\operatorname{Mod}(l+1, a, C)$. We have the holomorphic frame $\boldsymbol{v}^{\dagger}=\left(v^{\dagger, p, q} \mid p+q=l\right)$, given as follows:

$$
v^{\dagger, p, q}=\left(v^{\dagger, 1,0}\right)^{p} \cdot\left(v^{\dagger, 0,1}\right)^{q} .
$$

The $\mu$-connection $\mathbb{D}^{\dagger}$ is described as follows:

$$
\mathbb{D}^{\dagger} v^{\dagger, p, q}=q \cdot v^{\dagger, p+1, q-1} .
$$

The prolongment of $\operatorname{Mod}(l+1, a, C)^{\dagger}$ by the frame $\boldsymbol{v}^{\dagger}$ is same as the prolongment by increasing order 0 . We denote the prolongment by $\operatorname{Mod}(l+1, a, C)_{0}^{\dagger}$. The $\mu$-connection $\mathbb{D}^{\dagger}$ is of log type on $\operatorname{Mod}(l+$ $1, a, C)_{0}^{\dagger}$. The residue can be obtained from (12).

We have the $C^{\infty}$-frame $\boldsymbol{v}^{\prime \dagger}=\left(v^{\prime \dagger, p, q} \mid p+q=l\right)$, given as follows:

$$
v^{\prime \dagger, p, q}=y^{\frac{p-q}{2}} \cdot v^{\dagger, p, q} .
$$

Then we have the transformation matrices $B_{0}^{\dagger}=\left(B_{0 i, q}^{\dagger}\right)$ and $B_{0}^{\prime \dagger}=$ $\left(B_{0 i, q}^{\prime \dagger}\right)$ defined as follows:

$$
v^{\dagger, l-q, q}=\sum_{i} B_{0, i, q}^{\dagger} \cdot v^{\dagger, l-i, i}, \quad v^{\prime \dagger, l-q, q}=\sum_{i} B_{0, i, q}^{\prime \dagger} \cdot v^{\dagger, l-i, i} .
$$


Remark 3.2. Note that the rules of the subscripts are slightly different in the cases $B_{0}^{\dagger}, B_{0}^{\prime \dagger}$ and $B_{0}$ and $B_{0}^{\prime}$. Briefly speaking, the order is reversed.

We have the following formulas by the same calculation:

$$
B_{0, i, q}^{\dagger}:= \begin{cases}0 & (i<q) \\ c(l-q, i-q) \cdot \mu^{i-q} \cdot(-y-c)^{-i+q} & (i \geq q) .\end{cases}
$$

And thus we have the following:

$$
B_{0, i, q}^{\prime \dagger}:= \begin{cases}0 & (i<q) \\ c(l-q, i-q) \cdot \mu^{i-q} \cdot y^{i-q} \cdot(-y-c)^{-i+q} & (i \geq q),\end{cases}
$$

and

$$
B_{0, i, q}^{\prime \dagger-1}:= \begin{cases}0 & (i<q) \\ c(l-q, i-q) \cdot \mu^{i-q} \cdot y^{i-q}(y+c)^{-i+q} & (i \geq q) .\end{cases}
$$

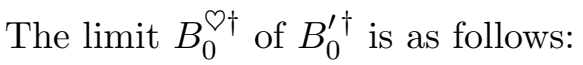

$$
\begin{aligned}
& B_{0, i, q}^{\mathrm{\dagger}}:= \begin{cases}0 & (i<q) \\
c(l-q, i-q) \cdot \mu^{i-q} & (i \geq q),\end{cases}
\end{aligned}
$$

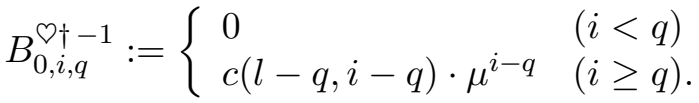

\subsubsection{Limit of $\operatorname{Mod}(l+1, a, C)$}

Let $\psi_{n}$ denote the morphism $\Delta \longrightarrow \Delta$ defined by $\psi_{n}(z)=z^{n}$. Later we will consider a 'limit' of harmonic bundles $\psi_{n}^{-1}\left(E, \bar{\partial}_{E}, \theta, h\right)$ for a harmonic bundle $\left(E, \bar{\partial}_{E}, \theta, h\right)$ on $\Delta^{*}$. Here we see what happens in the case of model bundles $\operatorname{Mod}(l, a, C)=\left(E_{l}, \theta_{l}, a \cdot h_{l}(C \cdot z)\right)$. We can assume that $C \in \mathbf{R}$. Recall that we have the natural frame $\boldsymbol{e}:=\boldsymbol{v}_{\mid \lambda=0}$ of $\operatorname{Mod}(l, a, C)$, that is we put $e^{p, q}(z):=v^{p, q}(0, z)$.

Let $F$ be a holomorphic vector bundle with a frame $\boldsymbol{u}=\left(u^{p, q} \mid p+q=\right.$ $l)$ over $\Delta^{*}$. We take a holomorphic isomorphism of $\psi_{n}^{-1}\left(E_{l}\right)$ to $F$ by the following correspondence:

$$
n^{-(l-2 i) / 2} \cdot \psi_{n}^{-1}\left(e^{i, l-i}\right) \longmapsto u^{i, l-i} .
$$

Then we obtain the Higgs fields $\theta^{(n)}$ and the metrics $h_{l}^{(n)}$ on $F$ for any $n$. 
We also have the natural frame $\boldsymbol{e}:=\left.\boldsymbol{v}\right|_{\lambda=0}$ of $\operatorname{Mod}\left(l, a, C^{1 / n}\right)$. By the frames $\boldsymbol{e}$ and $\boldsymbol{u}$, we have the isomorphism of $F$ and $E_{l}$. It induces the isomorphism of harmonic bundles $\left(F, \theta^{(n)}, h^{(n)}\right)$ and $\operatorname{Mod}\left(l, a, C^{1 / n}\right)$.

We take $\Theta^{(n)} \in \Gamma\left(\Delta^{*}, M(r) \otimes \Omega_{\Delta^{*}}^{1,0}\right)$ and $H^{(n)} \in C^{\infty}\left(\Delta^{*}, \mathcal{H}(r)\right)$ defined as follows:

$$
\theta^{(n)} \boldsymbol{u}=\boldsymbol{u} \cdot \Theta^{(n)}, \quad H^{(n)}=H\left(h^{(n)}, \boldsymbol{u}\right) .
$$

Then it is easy to see that the sequences $\left\{\Theta^{(n)}\right\}$ and $\left\{H^{(n)}\right\}$ are convergent on any compact subset $K \subset \Delta^{*}$, as the elements of $\Gamma\left(\Delta^{*}, M(r) \otimes\right.$ $\left.\Omega_{\Delta^{*}}^{1,0}\right)$ or $C^{\infty}\left(\Delta^{*}, \mathcal{H}(r)\right)$. In fact, we have $\Theta^{(n)}=\Theta$ and $H^{(n)}(z)=$ $a \cdot H\left(C^{1 / n} \cdot z\right)$. Here $\Theta$ and $H$ are given as follows:

$$
\theta_{l} \boldsymbol{e}=\boldsymbol{e} \cdot \Theta, \quad H=H\left(h_{l}, \boldsymbol{e}\right) .
$$

Thus $\Theta^{(n)}$ converges to $\Theta$ and $H^{(n)}$ converges to $a \cdot H$.

In the discussion above, we take a limit at $\lambda=0$. Clearly, we can take a limit at any $\lambda$. Namely, we put $\boldsymbol{w}(z):=\boldsymbol{v}(\lambda, z)$ for a fixed $\lambda$, and consider the frames $\boldsymbol{w}^{(n)}=\left(w^{(n) p, q}\right)$ defined by $w^{(n), p, q}:=$ $n^{-(p-q) / 2} \psi_{n}^{-1}\left(w^{p, q}\right)$. We have the $\lambda$-connection form $\psi_{n}^{-1}\left(\mathbb{D}^{\lambda}\right) \boldsymbol{w}^{(n)}=$ $\boldsymbol{w}^{(n)} \cdot \mathcal{A}^{(n)}$. Then $\left\{H\left(\psi_{n}^{-1}\left(h_{l}\right), \boldsymbol{w}^{(n)}\right)\right\}$ and $\left\{\mathcal{A}^{(n)}\right\}$ converges on any compact subset $K \subset \Delta^{*}$ as the sequences in $C^{\infty}\left(\Delta^{*}, \mathcal{H}(r)\right)$ and $\Gamma\left(\Delta^{*}, M(r) \otimes\right.$ $\left.\Omega_{\Delta^{*}}^{1,0}\right)$

\subsection{A convergence of a sequence of harmonic metrics}

\subsubsection{A convergence at $\lambda=1$}

Let $X$ be $\Delta^{* l} \times \Delta^{d-l}$, and $\left(E^{(n)}, \theta^{(n)}, h^{(n)}\right)$ be a harmonic bundles on $X$ such that $\operatorname{rank}\left(E^{(n)}\right)=r$. Recall that we have the deformed holomorphic bundles $\left(\mathcal{E}^{1(n)}, d^{\prime \prime 1(n)}, \mathbb{D}^{1(n)}, h^{(n)}\right)$ on $\mathcal{X}^{1}=\{1\} \times X \subset \mathcal{X}$. In this subsection, the metric and the measure of $X$ are $\sum_{i=1}^{n} d z_{i} \cdot d \bar{z}_{i}$ and $\prod_{i=1}^{n}\left|d z_{i} \cdot d \bar{z}_{i}\right|$.

Assume that we have frames $\boldsymbol{w}^{(n)}=\left(w_{1}^{(n)}, \ldots, w_{r}^{(n)}\right)$ of $\mathcal{E}^{1(n)}$, and the following holds:

\section{Condition 3.1.}

1. We have the connection forms $A^{(n)} \in \Gamma\left(X, M(r) \otimes \Omega_{X}^{1,0}\right)$ determined by $\mathbb{D}^{1(n)} \boldsymbol{w}^{(n)}=\boldsymbol{w}^{(n)} A^{(n)}$. Then the sequence $\left\{A^{(n)}\right\}$ converges to $A^{(\infty)} \in \Gamma\left(X, M(r) \otimes \Omega_{X}^{1,0}\right)$ on any compact subset $K \subset X$. 
2. We put $H^{(n)}:=H\left(h^{(n)}, \boldsymbol{w}^{(n)}\right) \in C^{\infty}(X, \mathcal{H}(r))$. On any compact subset $K \subset X, H^{(n)}$ and $H^{(n)-1}$ are bounded independently of $n$. Namely we have a constant $C_{K}$ depending on $K$ such that $\left|H^{(n)}\right|<C_{K}$ and $\left|H^{(n)-1}\right|<C_{K}$.

3. On any compact subset $K \subset X$, the norms $\left|\theta^{(n)}\right|_{h^{(n)}}$ are bounded independently of $n$.

The element $\Theta^{(n)} \in C^{\infty}\left(X, M(r) \otimes \Omega_{X}^{1,0}\right)$ is determined by the following relation:

$$
\theta^{(n)} \boldsymbol{w}=\boldsymbol{w} \cdot \Theta^{(n)}
$$

The following lemma is easy to see.

Lemma 3.19. Under Condition 2, Condition 3 is equivalent to the following: On any compact subset $K \subset X,\left|\Theta^{(n)}\right|$ are bounded independently of $n$.

Proposition 3.1. Let $X,\left(E^{(n)}, \theta^{(n)}, h^{(n)}\right)$ and $\boldsymbol{w}^{(n)}$ be as above. Then we can take a subsequence $\left\{n_{i}\right\}$ of $\{n\}$ such that the sequences $\left\{H^{\left(n_{i}\right)}\right\},\left\{H^{\left(n_{i}\right)-1}\right\}$ and $\left\{\Theta^{\left(n_{i}\right)}\right\}$ are convergent on any compact subset $K$ in the $L_{l}^{p}$-sense. Here $p$ is a sufficiently large real number, and $l$ is an arbitrary large real number.

Proof. We will use the following lemma without mention.

Lemma 3.20. Let $K$ be a compact subset of $X$.

1. Let $\left\{f_{n}\right\}$ be a bounded sequence in $L_{l}^{p}(X)$ such that all of $f_{n}$ vanish on $X-K$.

Assume that the sequence $\left\{\bar{\partial}\left(f_{n}\right)\right\}$ are bounded in $L_{l}^{p}(X)$. Then $\left\{f_{n}\right\}$ is bounded in $L_{l+1}^{p}(X)$.

Similarly, when the sequence $\left\{\partial\left(f_{n}\right)\right\}$ is bounded in $L_{l}^{p}(X)$, then $\left\{f_{n}\right\}$ is bounded in $L_{l+1}^{p}(X)$.

2. Let $\left\{f_{n}\right\}$ be a sequence of $L_{l}^{p}(X)$ satisfying that any $f_{n}$ vanish on $X-K$, and that $f_{n}$ converges to $g$ in $L_{l}^{p}(X)$.

Assume that $\left\{\bar{\partial} f_{n}\right\}$ converges to $\bar{\partial} g$ in $L_{l}^{p}(X)$. Then $\left\{f_{n}\right\}$ converges to $g_{n}$ in $L_{l+1}^{p}(X)$.

Similarly when $\left\{\partial f_{n}\right\}$ converges to $\partial g$ in $L_{l}^{p}(X)$, then $\left\{f_{n}\right\}$ converges to $g_{n}$ in $L_{l+1}^{p}(X)$. 
Proof. Consider the first claim. We only have to show the boundedness of $\left\{\partial f_{n}\right\}$ in $L_{l}^{p}(X)$. Let $D$ be a differential operator with constant coefficient whose degree is less than $l$. We have the following equality:

$$
\int\left(\partial D f_{n}, \partial D f_{n}\right)=\int\left(\Delta D f_{n}, D f_{n}\right)=\int\left(\bar{\partial} D f_{n}, \bar{\partial} D f_{n}\right) .
$$

Thus the $L_{l}^{p}$-boundedness of $\left\{\bar{\partial} f_{n}\right\}$ implies that the $L_{l}^{p}$-boundedness of $\left\{\partial f_{n}\right\}$.

The second claim can be shown similarly. Thus the proof of Lemma 3.20 is completed. q.e.d.

Let us return to the proof of Proposition 3.1. The elements $\Theta^{\dagger(n)} \in$ $C^{\infty}\left(X, M(r) \otimes \Omega_{X}^{0,1}\right)$ are determined by the following condition:

$$
\theta^{(n) \dagger} \boldsymbol{w}^{(n)}=\boldsymbol{w}^{(n)} \Theta^{(n) \dagger} .
$$

Then we have the following relations:

$$
\Theta^{(n) \dagger}=\bar{H}^{(n)-1} \cdot{ }^{t} \bar{\Theta}^{(n)} \cdot \bar{H}^{(n)} .
$$

Hence we also have the boundedness of the sequence $\left\{\Theta^{(n) \dagger}\right\}$ on any compact subset $K \subset X$.

Let $\nabla^{(n)}$ be the unitary connection of $\left(\mathcal{E}^{1(n)}, d^{\prime \prime}(n), h^{(n)}\right)$. Then we have the relation

$$
\nabla^{(n)}=\bar{\partial}_{E^{(n)}}+\theta^{\dagger(n)}+\partial_{E^{(n)}}-\theta^{(n)}=\mathbb{D}^{1(n)}-2 \theta^{(n)} .
$$

We determine the element $B^{(n)} \in C^{\infty}\left(X, M(r) \otimes \Omega_{X}^{1,0}\right)$ by the relation $\nabla^{(n)} \boldsymbol{w}^{(n)}=\boldsymbol{w}^{(n)} \cdot B^{(n)}$. Then we have the following equalities by definitions:

$$
B^{(n)}=A^{(n)}-2 \Theta^{(n)}, \quad \text { i.e., } \quad \Theta^{(n)}=\frac{1}{2}\left(A^{(n)}-B^{(n)}\right) .
$$

On the other hand, we have the following relation:

$$
B^{(n)}=\left(H^{(n)}\right)^{-1} \partial H^{(n)}, \quad \text { i.e., } \quad \partial H^{(n)}=H^{(n)} \cdot B^{(n)} .
$$

Pick a compact subset $K \subset X$, and pick compact subsets $K_{1}$ and $K_{2}$ of $X$ such that $K$ is contained in the interior of $K_{1}$, and that $K_{1}$ is contained in the interior of $K_{2}$. We can pick an element $\varphi \in C^{\infty}(X, \mathbf{R})$, satisfying the following:

$$
0 \leq \varphi(x) \leq 1, \quad \varphi(x)= \begin{cases}1 & (x \in K) \\ 0 & \left(x \notin K_{2}\right)\end{cases}
$$

Take a sufficiently large number $p$ satisfying the following: 
Let $l$ be any real number larger than 1 . For any elements $f, g \in L_{l}^{p}(X)$, the product $f \cdot g$ is contained in $L_{l}^{p}(X)$.

Such $p$ can be taken, depending only on $\operatorname{dim} X$.

We need some equalities.

Lemma 3.21. We have the following equality:

$$
\bar{\partial}\left(\varphi^{m} \Theta^{(n)}\right)=\varphi^{m} \cdot\left[\Theta^{(n)}, \Theta^{(n) \dagger}\right]+m \cdot \bar{\partial} \varphi \cdot \varphi^{m-1} \cdot \Theta^{(n)} .
$$

Proof. We have $\bar{\partial}_{E^{(n)}}\left(\theta^{(n)}\right)=0$, in other words, $\left(d^{\prime 1(n)}-\theta^{(n) \dagger}\right)\left(\theta^{(n)}\right)$ $=0$. (Recall that we have $d^{\prime \prime 1}=\bar{\partial}_{E}+\theta^{\dagger}$, by definition for a harmonic bundle, in general.) It is reworded as follows:

$$
\bar{\partial} \Theta^{(n)}=\left[\Theta^{(n) \dagger}, \Theta^{(n)}\right] .
$$

The equality (21) follows immediately.

q.e.d.

Lemma 3.22. We have the following equality:

$$
\partial\left(\varphi^{m} \cdot H^{(n)}\right)=m \cdot(\partial \varphi) \cdot \varphi^{m-1} \cdot H^{(n)}+\varphi^{m} \cdot H^{(n)}\left(A^{(n)}-2 \Theta^{(n)}\right) .
$$

Proof. The equality (22) follows from (20) and the equality $B^{(n)}$ $=A^{(n)}-2 \Theta^{(n)}$.

q.e.d.

Lemma 3.23. For nonnegative integers $k$ and $l$, we have the following equality:

$$
\begin{aligned}
\partial\left(\varphi^{k+l} \cdot H^{(n)-1}\right)=(k+2 l) & \cdot \partial \varphi \cdot \varphi^{k+l-1} \cdot H^{(n)-1} \\
& -\varphi^{k} \cdot H^{(n)-1} \cdot \partial\left(\varphi^{l} \cdot H\right) \cdot H^{(n)-1} .
\end{aligned}
$$

Proof. We have the following direct calculation. We omit $(n)$ for simplicity, and we put $k+l=m$

$$
\begin{aligned}
& \partial\left(\varphi^{m} H^{-1}\right)=m \cdot \partial \varphi \cdot \varphi^{m-1} \cdot H^{-1}-\varphi^{m} \cdot H^{-1} \cdot \partial(H) \cdot H^{-1} \\
& =m \cdot \partial \varphi \cdot \varphi^{m-1} \cdot H^{-1}-\varphi^{k} \cdot H^{-1}\left(\partial\left(\varphi^{l} \cdot H\right)-\partial \varphi^{l} \cdot H\right) H^{-1} \\
& \quad=(m+l) \cdot \partial \varphi \cdot \varphi^{m-1} \cdot H^{-1}-\varphi^{k} \cdot H^{-1} \partial\left(\varphi^{l} \cdot H\right) H^{-1}
\end{aligned}
$$

Thus we are done.

q.e.d. 
Lemma 3.24. We have the following equation:

$$
\varphi^{k+l+m} \Theta^{(n) \dagger}=\left(\varphi^{k} \bar{H}^{(n)-1}\right) \cdot\left(\varphi^{l} \cdot{ }^{t} \bar{\Theta}^{(n)}\right) \cdot\left(\varphi^{m} \cdot \bar{H}^{(n)}\right) .
$$

Proof. The equality follows immediately from (18). q.e.d.

Let us return to the proof of Proposition 3.1. We use the standard boot strapping. We divide the argument into some lemmas.

\section{Lemma 3.25.}

- The sequences $\left\{\varphi \cdot \Theta^{(n)}\right\}$ and $\left\{\varphi^{3} \cdot \Theta^{\dagger(n)}\right\}$ are bounded in $L_{1}^{p}(X)$.

- The sequences $\left\{\varphi^{2} \cdot H^{(n)}\right\}$ and $\left\{\varphi^{4} \cdot H^{(n)-1}\right\}$ are bounded in $L_{2}^{p}(X)$.

Proof. It is clear that $\varphi \cdot \Theta^{(n)}$ are bounded in $L^{p}(X)$ independently of $n$. Then $\bar{\partial}\left(\varphi \cdot \Theta^{(n)}\right)=\varphi\left[\Theta^{(n) \dagger}, \Theta^{(n)}\right]+\bar{\partial} \varphi \cdot \Theta^{(n)}$ are bounded independently of $n$. Thus $\varphi \cdot \Theta^{(n)}$ are bounded in $L_{1}^{p}(X)$.

It is easy to see that $\varphi \cdot H^{(n)}$ are bounded in $L^{p}(X)$. Thus $\partial(\varphi$. $\left.H^{(n)}\right)=\partial \varphi \cdot H^{(n)}+\varphi \cdot H^{(n)}\left(A^{(n)}-2 \Theta^{(n)}\right)$ are bounded in $L^{p}(X)$. It implies that $\varphi \cdot H^{(n)}$ are bounded in $L_{1}^{p}(X)$. Then $\partial\left(\varphi^{2} \cdot H^{(n)}\right)=$ $2 \partial \varphi \cdot H^{(n)}+\varphi \cdot H^{(n)} \cdot\left(\varphi \cdot A^{(n)}-2 \varphi \cdot \Theta^{(n)}\right)$ are bounded in $L_{1}^{p}(X)$. Thus $\varphi^{2} \cdot H^{(n)}$ are bounded in $L_{2}^{p}(X)$.

It is easy to see that $\varphi \cdot H^{(n)-1}$ are bounded in $L^{p}(X)$. Then $\partial(\varphi$. $\left.H^{(n)-1}\right)=\partial \varphi \cdot H^{(n)-1}-\varphi \cdot H^{(n)-1} \partial H^{(n)} \cdot H^{(n)-1}$ are bounded in $L^{p}(X)$. Thus $\varphi \cdot H^{(n)-1}$ are bounded in $L_{1}^{p}(X)$. We have

$$
\begin{aligned}
\partial\left(\varphi^{4} \cdot H^{(n)-1}\right)= & 6 \cdot \partial \varphi \cdot \varphi^{3} \cdot H^{-1} \\
& -\left(\varphi \cdot H^{(n)-1}\right) \cdot \partial\left(\varphi^{2} \cdot H^{(n)}\right) \cdot\left(\varphi \cdot H^{(n)-1}\right) .
\end{aligned}
$$

Thus $\partial\left(\varphi^{4} H^{(n)-1}\right)$ are bounded in $L_{1}^{p}(X)$, and hence $\varphi^{4} H^{(n)-1}$ are bounded in $L_{2}^{p}(X)$.

Clearly, $\varphi^{3} \cdot \Theta^{\dagger(n)}=\left(\varphi \cdot \bar{H}^{(n)-1}\right) \cdot\left(\varphi \cdot{ }^{t} \bar{\Theta}^{(n)}\right) \cdot\left(\varphi \cdot \bar{H}^{(n)}\right)$ are bounded in $L_{1}^{p}(X)$.

q.e.d.

\section{Lemma 3.26.}

- The sequences $\left\{\varphi^{4} \cdot \Theta^{(n)}\right\}$ and $\left\{\varphi^{24} \Theta^{\dagger(n)}\right\}$ are bounded in $L_{2}^{p}(X)$.

- The sequences $\left\{\varphi^{6} \cdot H^{(n)}\right\}$ and $\left\{\varphi^{14} \cdot H^{(n)-1}\right\}$ are bounded in $L_{3}^{p}(X)$. 
Proof. By using the formulas (21) and the boundedness in Lemma 3.25, we obtain the boundedness of $\bar{\partial}\left(\varphi^{4} \Theta^{(n)}\right)$ in $L_{1}^{p}(X)$. Hence we obtain the boundedness of $\varphi^{4} \Theta^{(n)}$ in $L_{2}^{p}(X)$.

Similarly, by using the formulas (22), (23) and the boundedness in Lemma 3.25, we obtain the boundedness of $\partial\left(\varphi^{6} H^{(n)}\right)$ and $\partial\left(\varphi^{14}\right.$. $\left.H^{(n)-1}\right)$ in $L_{3}^{p}(X)$.

Lastly we obtain the boundedness of $\varphi^{10} \cdot \Theta^{(n) \dagger}$ in $L_{2}^{p}(X)$. And thus we obtain the boundedness of $\varphi^{24} \cdot \Theta^{(n) \dagger}$ in $L_{2}^{p}(X)$. $\quad$ q.e.d.

Take a subsequence $\left\{n_{i}\right\}$ of $\{n\}$ satisfying the following:

- The sequences $\left\{\varphi^{4} \cdot \Theta^{\left(n_{i}\right)}\right\}$ and $\left\{\varphi^{24} \cdot \Theta^{\left(n_{i}\right) \dagger}\right\}$ are convergent in $L_{1}^{p}(X)$. The limit is denoted by $a$ and $d$ respectively.

- The sequences $\left\{\varphi^{6} \cdot H^{\left(n_{i}\right)}\right\}$ and $\left\{\varphi^{14} \cdot H^{\left(n_{i}\right)-1}\right\}$ are convergent in $L_{2}^{p}(X)$. The limit is denoted by $b$ and $c$ respectively.

Such subsequence $\left(n_{i}\right)$ can be taken due to the Sobolev's embedding theorem.

We take the sequences of natural numbers $\left\{\alpha_{l}\right\},\left\{\beta_{l}\right\},\left\{\gamma_{l}\right\}$ and $\left\{\delta_{l}\right\}$ as follows:

- $\alpha_{1}=4, \beta_{1}=6, \gamma_{1}=14$ and $\delta_{1}=24$.

- We have the relations:

$$
\begin{array}{ll}
\alpha_{l}=\alpha_{l-1}+\delta_{l-1}, & \beta_{l}=\beta_{l-1}+\alpha_{l}, \\
\gamma_{l}=2 \gamma_{l-1}+\beta_{l}, & \delta_{l}=\alpha_{l}+\beta_{l}+\gamma_{l} .
\end{array}
$$

Such relations determines the sequence of the numbers uniquely.

\section{Lemma 3.27.}

- The sequences $\left\{\varphi^{\alpha_{l}} \cdot \Theta^{\left(n_{i}\right)}\right\}$ and $\left\{\varphi^{\delta_{l}} \cdot \Theta^{\left(n_{i}\right) \dagger}\right\}$ are convergent in $L_{l}^{p}(X)$. The limits are $\varphi^{\alpha_{l}-2} \cdot a$ and $\varphi^{\delta_{l}-24} d$ respectively.

- The sequences $\left\{\varphi^{\beta_{l}} \cdot H^{\left(n_{i}\right)}\right\}$ and $\left\{\varphi^{\gamma_{l}} \cdot H^{\left(n_{i}\right)-1}\right\}$ are convergent in $L_{l+1}^{p}(X)$. The limits are $\varphi^{\beta_{l}-6} \cdot b$ and $\varphi^{\gamma_{l}-14} \cdot c$ respectively.

Proof. We use the induction on $l$. The assertions for $l=1$ hold because of our choice. We assume that the assertions for $l-1$ hold, and we will prove the assertions for $l$. 
Due to the formula (21), we have the following:

$$
\begin{aligned}
\bar{\partial}\left(\varphi^{\alpha_{l-1}+\delta_{l-1}} \cdot \Theta^{\left(n_{i}\right)}\right)= & {\left[\varphi^{\alpha_{l-1}} \Theta^{\left(n_{i}\right)}, \varphi^{\delta_{l-1}} \Theta^{\left(n_{i}\right) \dagger}\right] } \\
& +\left(\alpha_{l-1}+\delta_{l-1}\right) \cdot \bar{\partial} \varphi \cdot \varphi^{\delta_{l-1}-1} \cdot\left(\varphi^{\alpha_{l-1}} \cdot \Theta^{\left(n_{i}\right)}\right) .
\end{aligned}
$$

Thus we obtain the convergence of $\bar{\partial}\left(\varphi^{\alpha_{l}} \cdot \Theta^{\left(n_{i}\right)}\right)$ in $L_{l-1}^{p}(X)$. Thus we obtain the convergence of $\left\{\varphi^{\alpha_{l}} \Theta^{\left(n_{i}\right)}\right\}$ in $L_{l}^{p}(X)$.

We have the following:

$$
\begin{aligned}
\partial\left(\varphi^{\alpha_{l}+\beta_{l-1}} H^{\left(n_{i}\right)}\right)= & \left(\alpha_{l}+\beta_{l-1}\right) \cdot \partial(\varphi) \cdot \varphi^{\alpha_{l}+\beta_{l-1}-1} \cdot H^{\left(n_{i}\right)} \\
& +\left(\varphi^{\beta_{l-1}} H^{\left(n_{i}\right)}\right) \cdot\left(\varphi^{\alpha_{l}} \cdot A^{\left(n_{i}\right)}-\varphi^{\alpha_{l}} \cdot \Theta^{\left(n_{i}\right)}\right) .
\end{aligned}
$$

The convergence of the right-hand side is in $L_{l}^{p}(X)$. Thus we obtain the convergence of $\left\{\varphi^{\beta_{l}} \cdot H^{\left(n_{i}\right)}\right\}$ in $L_{l+1}^{p}(X)$.

We have the following:

$$
\begin{aligned}
& \partial\left(\varphi^{2 \gamma_{l-1}+\beta_{l}} H^{\left(n_{i}\right)-1}\right) \\
& =2\left(\gamma_{l-1}+\beta_{l}\right) \cdot \partial \varphi \cdot \varphi^{2 \gamma_{l-1}+\beta_{l}-1} \cdot\left(\varphi^{\gamma_{l-1}} \cdot H^{\left(n_{i}\right)-1}\right) \\
& \quad-\varphi^{\gamma_{l-1}} \cdot H^{\left(n_{i}\right)-1} \cdot \partial\left(\varphi^{\beta_{l}} \cdot H^{\left(n_{i}\right)}\right) \cdot \varphi^{\gamma_{l-1}} \cdot H^{\left(n_{i}\right)-1} .
\end{aligned}
$$

The convergence of the right-hand side is in $L_{l}^{p}(X)$, and thus the convergence of $\left\{\varphi^{\gamma_{l}} \cdot H^{\left(n_{i}\right)-1}\right\}$ is in $L_{l+1}^{p}(X)$.

Lastly we have the following:

$$
\varphi^{\alpha_{l}+\beta_{l}+\gamma_{l}} \cdot \Theta^{(n) \dagger}=\left(\varphi^{\gamma_{l}} \cdot \bar{H}^{\left(n_{i}\right)-1}\right) \cdot\left(\varphi^{\alpha_{l}} \cdot{ }^{t} \bar{\Theta}^{(n)}\right) \cdot\left(\varphi^{\beta_{l}} \cdot \bar{H}^{\left(n_{i}\right)}\right) .
$$

Thus the induction can proceed.

q.e.d.

By our choice of $\varphi$, we have $\varphi=1$ on $K$. Thus we obtain the convergence of $\left\{\Theta^{\left(n_{i}\right)}\right\},\left\{H^{\left(n_{i}\right)}\right\},\left\{H^{\left(n_{i}\right)-1}\right\}$ and $\left\{\Theta^{\dagger\left(n_{i}\right)}\right\}$ on $K$. By using a standard diagonal argument, we can take a sequence $\left\{n_{i}\right\}$ such that the sequences $\left\{\Theta^{\left(n_{i}\right)}\right\},\left\{H^{\left(n_{i}\right)}\right\},\left\{H^{\left(n_{i}\right)-1}\right\}$ and $\left\{\Theta^{\dagger\left(n_{i}\right)}\right\}$ converge on any compact subset $K \subset X$. Thus the proof of Proposition 3.1 is completed.

q.e.d.

Take a holomorphic bundle $F=\bigoplus \mathcal{O}_{X} \cdot e_{i}$ with the frame $\boldsymbol{e}=$ $\left(e_{i}\right)$. The frames $\boldsymbol{w}^{(n)}$ and $\boldsymbol{e}$ induce the holomorphic isomorphism $\Phi_{n}:\left(\mathcal{E}^{(n) 1}, \boldsymbol{w}^{(n)}\right) \longrightarrow(F, \boldsymbol{e})$. Then we obtain the sequence of the metrics $\left\{h^{(n)}\right\}$, (non-holomorphic) Higgs fields $\left\{\theta^{(n)}\right\}$, the conjugates $\left\{\theta^{(n) \dagger}\right\}$ and the holomorphic structures $\left\{\bar{\partial}^{(n)}:=d_{F}^{\prime \prime}-\theta^{(n) \dagger}\right\}$ on $F$. Take 
the subsequence $\left\{n_{i}\right\}$ as in Proposition 3.1. Then the corresponding sequences converge, and thus we obtain the limits $h^{(\infty)}, \theta^{(\infty)}, \theta^{(\infty) \dagger}$, $\bar{\partial}^{(\infty)}$ on $F$.

Definition 3.2. The tuple of limits $\left(F, \bar{\partial}^{(\infty)}, \theta^{(\infty)}, h^{(\infty)}\right)$ will be called the limiting harmonic bundle of the sequence $\left\{\left(E^{(n)}, \theta^{(n)}, h^{(n)}\right)\right\}$, although we do not have the uniqueness of the limit, in general.

Remark 3.3. In this subsubsection, we discussed the convergence at $\lambda=1$. Clearly, the same argument works if $\lambda \neq 0$. The key equality is (19).

In the case $\lambda=0$, Conditions 1 and 2 in Condition 3.1 imply Condition 3. Hence the convergence of $\theta$ is obtained from the definition. But we do not have the relation of the metric connection and $\theta$ at $\lambda=0$. Thus the argument to treat the metric breaks.

\subsubsection{The dependence on the holomorphic frames $w^{(n)}$}

Let $\dot{\boldsymbol{w}}^{(n)}$ be holomorphic frames of $\mathcal{E}^{(n) 1}$ satisfying Condition 3.1. We take a holomorphic vector bundle $\dot{F}=\bigoplus_{i=1}^{r} \mathcal{O}_{X} \cdot \dot{e}_{i}$. The frames $\dot{\boldsymbol{w}}^{(n)}$ and $\dot{\boldsymbol{e}}$ induces the isomorphism $\dot{\Phi}_{n}:\left(\mathcal{E}^{(n) 1}, \dot{\boldsymbol{w}}^{(n)}\right) \longrightarrow(\dot{F}, \dot{\boldsymbol{e}})$. Then we obtain the sequences $\left\{\dot{h}^{(n)}\right\},\left\{\dot{\theta}^{(n)}\right\},\left\{\dot{\theta}^{(n) \dagger}\right\}$, and $\left\{\dot{\bar{\partial}}^{(n)}\right\}$. We take a subsequence $\left\{\dot{n}_{i}\right\}$ of $\{n\}$ such that the corresponding subsequences are convergent to $\dot{h}^{(\infty)}, \dot{\theta}^{(\infty)}, \dot{\theta}^{(\infty) \dagger}$ and $\dot{\bar{\partial}}^{(\infty)}$ respectively. We can assume that $\left\{\dot{n}_{i}\right\}$ is a subsequence of $\left\{n_{i}\right\}$.

Lemma 3.28. We can take a subsequence $\left\{\dot{n}_{i}\right\}$ such that the limiting harmonic bundles $\left(\dot{F}, \dot{\bar{\partial}}^{(\infty)}, \dot{\theta}^{(\infty)}, \dot{h}^{(\infty)}\right)$ is isomorphic to $\left(F, \bar{\partial}^{(\infty)}\right.$, $\left.\theta^{(\infty)}, h^{(\infty)}\right)$.

Proof. We have the sequence of holomorphic frames $\Phi_{n}\left(\dot{\boldsymbol{w}}^{(n)}\right)$ of $F$. Due to Condition 2 in Condition 3.1 for $\boldsymbol{w}^{(n)}$ and $\dot{\boldsymbol{w}}^{(n)}$, we can take a subsequence $\left\{n_{i_{j}}\right\}$ of $\left\{n_{i}\right\}$ such that $\Phi_{n_{i_{j}}}\left(\dot{\boldsymbol{w}}^{\left(n_{i_{j}}\right)}\right)$ converges to a holomorphic frame of $F$. We can assume that $\left\{n_{i_{j}}\right\}$ is a subsequence of $\left\{\dot{n}_{i}\right\}$. We can replace $\left\{\dot{n}_{i}\right\}$ by $\left\{n_{i_{j}}\right\}$, and thus we can assume that $\left\{\Phi_{\dot{n}_{i}}\left(\dot{\boldsymbol{w}}^{\left(\dot{n}_{i}\right)}\right)\right\}$ are convergent.

The frames $\boldsymbol{w}^{(n)}$ and $\dot{\boldsymbol{w}}^{(n)}$ induce the isomorphism $G^{(n)}$ of the harmonic bundles $\left(F, \bar{\partial}^{(n)}, \theta^{(n)}, h^{(n)}\right)$ and $\left(\dot{F}, \dot{\bar{\partial}}^{(n)}, \dot{\theta}^{(n)}, \dot{h}^{(n)}\right)$, by our construction. For the subsequence $\left\{\dot{n}_{i}\right\}$ above, the sequence $G^{\left(\dot{n}_{i}\right)}$ converges to a holomorphic isomorphism $G^{(\infty)}$. Clearly, $G^{(\infty)}$ gives an isomor- 
phism of limiting harmonic bundles $\left(F, \bar{\partial}^{(\infty)}, \theta^{(\infty)}, h^{(\infty)}\right)$ and $\left(\dot{F}, \dot{\bar{\partial}}^{(\infty)}\right.$, $\left.\dot{\theta}^{(\infty)}, \dot{h}^{(\infty)}\right)$.

Lemma 3.28 is reworded as follows.

Corollary 3.6. Let $\boldsymbol{w}^{(n)}$ and $\dot{\boldsymbol{w}}^{(n)}$ be two holomorphic frames of $\mathcal{E}^{(n) 1}$ satisfying Condition 3.1. Then we can take a subsequence $\left\{n_{i}\right\}$ of $\{n\}$ such that we have the natural isomorphism between the limiting harmonic bundles for the frames $\boldsymbol{w}^{(n)}$ and $\dot{\boldsymbol{w}}^{(n)}$.

\subsubsection{Convergence of a sequence of the frames of the de- formed holomorphic bundles}

Continue to use the notation in the previous subsubsection. From harmonic bundles $\left(F, \bar{\partial}^{(n)}, h^{(n)}, \theta^{(n)}\right)$, we obtain the deformed holomorphic bundles $\mathcal{F}^{(n)}=\left(p_{\lambda}^{-1} F, d^{\prime \prime}(n)\right)$ over $\mathcal{X}$. Note that $\mathcal{F}^{(n) 1}$ over $\mathcal{X}^{1}$ is same as $\left(F, d_{F}^{\prime \prime}\right)$ by our construction. Take a subsequence $\left\{n_{i}\right\}$ as above. Since we have $d^{\prime \prime(n)}=\bar{\partial}^{(n)}+\lambda \cdot \theta^{(n) \dagger}+\bar{\partial}_{\lambda}=d_{F}^{\prime \prime}+(\lambda-1) \cdot \theta^{(n) \dagger}+\bar{\partial}_{\lambda}$, and since the sequence of the Higgs fields $\left\{\theta^{\left(n_{i}\right)}\right\}$ and the metrics $\left\{h^{\left(n_{i}\right)}\right\}$ are convergent, the sequence $\left\{d^{\prime \prime\left(n_{i}\right)}\right\}$ converges to $d^{\prime \prime(\infty)}=d_{F}^{\prime \prime}+(\lambda-1) \theta^{(\infty) \dagger}+\bar{\partial}_{\lambda}$.

Let $\left\{f^{(n)}\right\}$ be a sequence of holomorphic sections of $\mathcal{F}^{(n)}$ over $\mathcal{X}$. Namely $f^{(n)}$ are elements of $C^{\infty}\left(\mathcal{X}, p_{\lambda}^{-1} F\right)$ satisfying $d^{\prime \prime}(n)\left(f^{(n)}\right)=0$. Assume the following:

- For any compact subset $K \subset \mathcal{X}$, we have a constant $C_{K}>0$ such that $\left|h^{(n)}\left(f^{(n)}, f^{(n)}\right)\right|<C_{K}$ for any $n$.

This is equivalent to the following:

- We have the $C^{\infty}$-functions $f_{i}^{(n)}$ determined by $f^{(n)}=\sum f_{i}^{(n)}$. $p_{\lambda}^{-1} e_{i}$. For any compact subset $K \subset \mathcal{X}$, we have a constant $C_{K}>0$ such that $\left|f_{i}^{(n)}\right|<C_{K}$.

Lemma 3.29. Let $f^{(n)}$ be as above. We can take a subsequence $\left\{n_{i_{j}}\right\}$ of $\left\{n_{i}\right\}$ such that the sequence $\left\{f^{\left(n_{i_{j}}\right)}\right\}$ is convergent to a holomorphic section of $\mathcal{F}^{(\infty)}$ on any compact subset $K$ in the $L_{l}^{p}$-sense. Here $p$ is a sufficiently large real number, and $l$ is an arbitrary large real number.

Proof. We can use an argument similar to the proof of Proposition 3.1.

q.e.d. 
Let $\boldsymbol{v}^{(n)}=\left(v_{1}^{(n)}, \ldots, v_{r}^{(n)}\right)$ be frames of $\mathcal{F}^{(n)}$ over $\mathcal{X}$. Assume that we have positive constants $C_{K, 1}$ and $C_{K, 2}$ for any compact subset satisfying the following:

$$
h^{(n)}\left(v_{i}^{(n)}, v_{i}^{(n)}\right)<C_{K, 1}, \quad h^{(n)}\left(\Omega\left(\boldsymbol{v}^{(n)}\right), \Omega\left(\boldsymbol{v}^{(n)}\right)\right)>C_{K, 2} .
$$

Lemma 3.30. We have a subsequence $\left\{n_{i_{j}}\right\}$ of $\left\{n_{i}\right\}$ such that the sequence $\left\{\boldsymbol{v}^{(n)}\right\}$ converges to a holomorphic frame $\left\{\boldsymbol{v}^{(\infty)}\right\}$ of $\mathcal{F}^{(\infty)}$ on any compact subset $K$ in the $L_{l}^{p}$-sense.

Proof. By the boundedness of $h^{(n)}\left(v_{i}^{(n)}, v_{i}^{(n)}\right)$ and Lemma 3.29, we have a subsequence $\left\{n_{i_{j}}\right\}$ such that the sequence of sections $\left\{v_{i}^{\left(n_{i_{j}}\right)}\right\}$ converges to a holomorphic section $v_{i}^{(\infty)}$. We only have to show that $\boldsymbol{v}^{(\infty)}=\left(v_{i}^{(\infty)}\right)$ gives a holomorphic frame.

On any compact subset $K$, we have the estimate

$$
h^{(n)}\left(\Omega\left(\boldsymbol{v}^{(n)}\right), \Omega\left(\boldsymbol{v}^{(n)}\right)\right)>C_{K, 2} .
$$

Thus we obtain $h^{(\infty)}\left(\Omega\left(\boldsymbol{v}^{(\infty)}\right), \Omega\left(\boldsymbol{v}^{(\infty)}\right)\right)>C_{K, 2}$. Hence $\boldsymbol{v}^{(\infty)}$ gives a frame. q.e.d.

Corollary 3.7. Let $\boldsymbol{v}^{(n)}$ be a holomorphic frame of $\mathcal{F}^{(n)}$ satisfying the following:

- For any compact subset $K$, the hermitian matrices $H\left(h^{(n)}, \boldsymbol{v}^{(n)}\right)$ and the inverse $H\left(h^{(n)}, \boldsymbol{v}^{(n)}\right)^{-1}$ are bounded independently of $n$.

Then we can take a subsequence $\left\{n_{i_{j}}\right\}$ of $\left\{n_{i}\right\}$ such that the sequence $\left\{\boldsymbol{v}^{\left(n_{i_{j}}\right)}\right\}$ converges to a holomorphic frame of $\mathcal{F}^{(\infty)}$.

Let $\boldsymbol{v}^{(n)}$ be a holomorphic frame of $\mathcal{F}^{(n)}$. Then we have the transformation matrices $B^{(n)} \in C^{\infty}(\mathcal{X}, M(r))$ determined by the relation $\boldsymbol{v}^{(n)}=p_{\lambda}^{-1} \boldsymbol{e} \cdot B^{(n)}$. Corollary 3.7 can be reworded as follows:

Corollary 3.8. Let $\boldsymbol{v}^{(n)}$ be a holomorphic frame of $\mathcal{F}^{(n)}$. Assume that the transformation matrices $B^{(n)}$ and their inverses $B^{(n)-1}$ are bounded independently of $n$, on any compact subset $K$. Then we can take a subsequence $\left\{n_{i_{j}}\right\}$ of $\left\{n_{i}\right\}$ such that $\left\{\boldsymbol{v}^{\left(n_{i_{j}}\right)}\right\}$ converges to a holomorphic frame of $\mathcal{F}^{(\infty)}$. 


\subsubsection{Convergence at any $\lambda$ and $\mu$}

We reword the result as follows: Let $\left(E^{(n)}, \bar{\partial}_{E^{(n)}}, h^{(n)}, \theta^{(n)}\right)$ be a sequence of harmonic bundles over $X$. We have the deformed holomorphic bundles $\left(\mathcal{E}^{(n)}, d^{\prime \prime(n)}, \mathbb{D}^{(n)}, h^{(n)}\right)$ over $\mathcal{X}$. Let $U_{1}$ be an open subset of $\mathbf{C}_{\lambda}$. We assume that $U_{1}$ contains $1 \in \mathbf{C}_{\lambda}$ for simplicity. We put $\mathcal{U}:=U_{1} \times X \subset \mathcal{X}$.

Let $\boldsymbol{v}^{(n)}$ be a holomorphic frame of $\mathcal{E}^{(n)}$ over $\mathcal{U}$.

\section{Condition 3.2.}

1. We have the $\lambda$-connection form $\mathcal{A}^{(n)} \in \Gamma\left(\mathcal{U}, M(r) \otimes p_{\lambda}^{*} \Omega_{X}^{1,0}\right)$, such that $\mathbb{D}\left(\boldsymbol{v}^{(n)}\right)=\boldsymbol{v}^{(n)} \cdot \mathcal{A}^{(n)}$. Then the sequence $\left\{\mathcal{A}^{(n)}\right\}$ converges to $\mathcal{A}^{(\infty)} \in \Gamma\left(\mathcal{U}, M(r) \otimes p_{\lambda}^{*} \Omega_{X}^{1,0}\right)$ on any compact subset $K \subset \mathcal{U}$.

2. We put $H^{(n)}:=H\left(h^{(n)}, \boldsymbol{v}^{(n)}\right) \in C^{\infty}(\mathcal{U}, \mathcal{H}(r))$. Then $H^{(n)}$ and $H^{(n)-1}$ are bounded independently of $n$, on any compact subset $K \subset \mathcal{U}$.

3. On any compact subset $K$, we have a positive constant $C_{K}$ such that $\left|\theta^{(n)}\right|_{h^{(n)}}<C_{K}$.

The element $\Theta^{(n)} \in C^{\infty}\left(\mathcal{U}, M(r) \otimes p_{\lambda}^{*} \Omega_{X}^{1,0}\right)$ is determined by the relation $\theta^{(n)}\left(\boldsymbol{v}^{(n)}\right)=\boldsymbol{v}^{(n)} \cdot \Theta^{(n)}$.

Proposition 3.2. We can take a subsequence $\left\{n_{i}\right\}$ such that the sequences $\left\{H^{\left(n_{i}\right)}\right\},\left\{H^{\left(n_{i}\right)-1}\right\}$ and $\left\{\Theta^{\left(n_{i}\right)}\right\}$ are convergent in $L_{l}^{p}$ on any compact subset $K$.

Proof. When we take the restriction of $\boldsymbol{v}^{(n)}$ to $\lambda=1$, then Condition 3.1 is satisfied. Thus we can take $\left\{n_{i}\right\}$ as in Proposition 3.1. Then we take a subsequence $\left\{n_{i_{j}}\right\}$ as in Corollary 3.7. By construction, it is clear that the sequences $\left\{H^{\left(n_{i_{j}}\right)}\right\},\left\{H^{\left(n_{i_{j}}\right)-1}\right\}$ and $\left\{\Theta^{\left(n_{i_{j}}\right)}\right\}$ are convergent. We only have to replace $\left\{n_{i}\right\}$ with $\left\{n_{i_{j}}\right\}$. $\quad$ q.e.d.

Pick an element $\lambda \in U$. We denote the restriction of $\boldsymbol{v}^{(n)}$ to $\{\lambda\} \times X$ by $\boldsymbol{v}_{\mid \lambda}^{(n)}$. We take a holomorphic vector bundle $F=\bigoplus_{i=1}^{r} \mathcal{O}_{X} \cdot e_{i}$. By the frames $\boldsymbol{v}_{\mid \lambda}^{(n)}$ and $\boldsymbol{e}=\left(e_{i}\right)$, we have the holomorphic isomorphism $\Phi_{n, \lambda}:\left(\mathcal{E}^{(n) \lambda}, \boldsymbol{v}_{\mid \lambda}^{(n)}\right) \longrightarrow(F, \boldsymbol{e})$. Then we obtain the sequence of the metrics $\left\{h^{(n)}\right\}$, the (non-holomorphic) Higgs fields $\left\{\theta^{(n)}\right\}$, the conjugates $\left\{\theta^{(n) \dagger}\right\}$ and the holomorphic structures $\left\{\bar{\partial}^{(n)}:=d_{F}^{\prime \prime}-\lambda \theta^{(n) \dagger}\right\}$. Take a subsequence $\left\{n_{i}\right\}$ as in Proposition 3.2. Then we obtain the 
limits of the sequences $h^{(\infty)}, \theta^{(\infty)}, \theta^{(\infty) \dagger}$ and $\bar{\partial}^{(\infty)}$. The tuple of limits $\left(F, \bar{\partial}^{(\infty)}, \theta^{(\infty)}, h^{(\infty)}\right)$ is a harmonic bundle, called a limiting harmonic bundle.

By our construction, we can see that the limit is independent of a choice of $\lambda$, in the sense that we have the canonical isomorphisms between the limits, once we fix an appropriate subsequence.

Let $\left\{\boldsymbol{v}^{(n)}\right\}$ and $\left\{\boldsymbol{\boldsymbol { v }}^{(n)}\right\}$ be two sequences of holomorphic frames of $\mathcal{E}_{\mid \mathcal{U}}^{(n)}$ satisfying Condition 3.2. By an argument similar to that in Subsubsection 3.3.2, we can show the following.

Proposition 3.3. We have a subsequence $\left\{n_{i}\right\}$ of $\{n\}$ satisfying the following:

- We have the limiting harmonic bundles for $\left\{\boldsymbol{v}^{\left(n_{i}\right)}\right\}$ and $\left\{\dot{\boldsymbol{v}}^{\left(n_{i}\right)}\right\}$.

- The limits are naturally isomorphic.

Consider the case $\mathcal{U}=\mathcal{X}$ for simplicity. We have the frame $\boldsymbol{v}^{(n) \dagger}$ of $\mathcal{E}^{(n) \dagger}$ over $\mathcal{X}^{\dagger}$. We denote the conjugate deformed holomorphic bundle of $\left(F, \bar{\partial}^{(n)}, \theta^{(n)}, h^{(n)}\right)$ by $\mathcal{F}^{(n) \dagger}$. The morphism $\Phi_{n}$ induces the holomorphic isomorphism $\mathcal{E}^{(n) \dagger} \longrightarrow \mathcal{F}^{(n) \dagger}$, which we also denote by $\Phi_{n}$. Thus we obtain the sequence of holomorphic frames $\left\{\Phi_{n}\left(\boldsymbol{v}^{(n) \dagger}\right)\right\}$. The following lemma can be easily seen from our construction.

Lemma 3.31. The sequence $\left\{\Phi_{n_{i}}\left(\boldsymbol{v}^{\left(n_{i}\right) \dagger}\right)\right\}$ is convergent.

Pick $\mu \in \mathbf{C}_{\mu}$. We denote the restriction of $\boldsymbol{v}^{(n) \dagger}$ to $\{\mu\} \times X^{\dagger}$ by $\boldsymbol{v}_{\mid \mu}^{(n) \dagger}$. We take a holomorphic bundle $F^{\dagger}=\bigoplus_{i=1}^{r} \mathcal{O}_{X^{\dagger}} \cdot e_{i}^{\dagger}$. Due to the frames $\boldsymbol{v}_{\mid \mu}^{(n) \dagger}$ and $\boldsymbol{e}^{\dagger}=\left(e_{i}^{\dagger}\right)$, we have the holomorphic isomorphism $\left(\mathcal{E}^{(n) \dagger \mu}, \boldsymbol{v}_{\mid \mu}^{(n) \dagger}\right) \longrightarrow\left(F^{\dagger}, \boldsymbol{e}^{\dagger}\right)$. Thus we obtain the sequence of the hermitian metrics $\left\{h^{(n)}\right\}$, the Higgs fields $\left\{\theta^{\dagger(n)}\right\}$ on $X^{\dagger}$, the conjugates $\left\{\theta^{(n)}\right\}$, and the holomorphic structure $\left\{\partial^{(n)}=d_{F^{\dagger}}^{\prime \prime}-\mu \cdot \theta^{(n)}\right\}$. Due to Lemma 3.31, the corresponding subsequences for $\left\{n_{i}\right\}$ converge. The limit is independent of a choice of $\mu$ in the sense that we have the canonical isomorphism between the limits, once we fix an appropriate subsequence. Moreover the limit obtained from the frames $\left\{\boldsymbol{v}_{\mid \mu}^{(n) \dagger}\right\}$ are canonically isomorphic to the limit obtained from the frames $\left\{\boldsymbol{v}_{\mid \lambda}^{(n)}\right\}$. 


\section{Prolongation of the deformed holomorphic bundle}

\subsection{Prolongation by increasing orders}

Let $X$ be an $n$-dimensional complex manifold, and let $D=\bigcup_{i \in I} D_{i}$ be a simple normal crossing divisor.

Definition 4.1. Let $P$ be a point of $X$, and let $D_{i_{j}}(j=1, \ldots, l)$ be components of $D$ containing $P$. An admissible coordinate around $P$ is the tuple $(\mathcal{U}, \varphi)$ :

- $\mathcal{U}$ is an open subset of $X$ containing $P$.

- $\varphi$ is a holomorphic isomorphism $\mathcal{U} \longrightarrow \Delta^{n}=\left\{\left(z_{1}, \ldots, z_{n}\right)|| z_{i} \mid<\right.$ $1\}$ such that $\varphi(P)=(0, \ldots, 0)$, and $\varphi\left(D_{i_{j}}\right)=\left\{z_{j}=0\right\}$ for any $j=1, \ldots, l$.

Let $\left(E, \bar{\partial}_{E}, h\right)$ be a holomorphic bundle with a hermitian metric defined over $X-D$. Let $\boldsymbol{\alpha}=\left(\alpha_{i} \mid i \in I\right) \in \mathbf{R}^{I}$ be a tuple of real numbers.

Definition 4.2. Let $U$ be an open subset of $X$, and $s$ be an element of $\Gamma(U-D, E)$. We say that the increasing order of $s$ is less than $\boldsymbol{\alpha}$, if the following holds:

- Let $P$ be a point of $U$. Take an admissible coordinate $(\mathcal{U}, \varphi)$ around $P$. Let $\epsilon$ be any positive real number. Then we have a positive constant $C$ such that the following inequality holds on $\mathcal{U}$ :

$$
|s|_{h} \leq C \cdot \prod_{j=1}^{l}\left|z_{j}\right|^{\alpha_{i_{j}}-\epsilon} .
$$

In the case, it is described as $\operatorname{ord}(s) \leq \boldsymbol{\alpha}$.

Pick a tuple $\boldsymbol{\alpha}$. Then the $\mathcal{O}_{X}$-sheaf $E_{\leq \boldsymbol{\alpha}}$ is defined as follows: For any open subset $U \subset X$,

$$
\Gamma\left(U, E_{\leq \boldsymbol{\alpha}}\right):=\{s \in \Gamma(U-U \cap D, E) \mid \operatorname{ord}(s) \leq \boldsymbol{\alpha}\} .
$$

We often use the notation $E_{\boldsymbol{\alpha}}$ instead of $E_{\leq \alpha}$. The sheaf $E_{\boldsymbol{\alpha}}$ is called the prolongment of $E$ by an increasing order $\boldsymbol{\alpha}$.

Notation $\left({ }^{\diamond} E\right)$. In this paper, we are mainly interested in the case $\alpha_{i}=0$ for any $i$. In that case we will use the notation ${ }^{\diamond} E$. 
We also define the $\mathcal{O}_{X}$-sheaf $E_{<\boldsymbol{\alpha}}$ as follows:

$$
\begin{aligned}
\Gamma\left(U, E_{<\boldsymbol{\alpha}}\right):=\{s \in \Gamma(U-U \cap D, E) \mid \exists \epsilon>0, \\
\text { such that } \operatorname{ord}(s) \leq \boldsymbol{\alpha}+\epsilon \cdot \boldsymbol{\delta}\} .
\end{aligned}
$$

Here we put $\boldsymbol{\delta}=(1, \ldots, 1)$.

The Poincaré metrics $g_{1}$ on $\Delta$ and $g_{0}$ on $\Delta^{*}$ are given by the following formulas up to some minor modification:

$$
g_{1}=\frac{2 d z \cdot d \bar{z}}{\left(1-|z|^{2}\right)^{2}}, \quad g_{0}=\frac{2 d z \cdot d \bar{z}}{|z|^{2}\left(-\log |z|^{2}\right)^{2}}
$$

The associated Kahler forms $\omega_{1}$ and $\omega_{0}$ are as follows:

$$
\omega_{1}=\frac{\sqrt{-1} d z \cdot d \bar{z}}{\left(1-|z|^{2}\right)^{2}}, \quad \omega_{0}=\frac{\sqrt{-1} d z \cdot d \bar{z}}{|z|^{2}\left(-\log |z|^{2}\right)^{2}}
$$

As the metric on $\Delta^{* l} \times \Delta^{n-l}$, we have the metric and the Kahler form:

$$
g_{\mathbf{p}}:=\sum_{j=1}^{l} \pi_{j}^{*} g_{0}+\sum_{j=l+1}^{n} \pi_{j}^{*} g_{1}, \quad \omega_{\mathbf{p}}=\sum_{j=1}^{l} \pi_{j}^{*} \omega_{0}+\sum_{j=l+1}^{n} \pi_{j}^{*} \omega_{1} .
$$

Let $P$ be a point of $X$ and $(\mathcal{U}, \varphi)$ be an admissible coordinate around $P$. By the isomorphism $\varphi: \mathcal{U}-D \simeq \Delta^{* l} \times \Delta^{n-l}$, we take the Poincaré metric $g_{\mathbf{p}}$ on $\mathcal{U}-D$. The metric $h$ of $E$ and the metric $g_{\mathbf{p}}$ on $T(\mathcal{U}-D)$ induce the metric $(\cdot, \cdot)_{h, g_{\mathbf{p}}}$ of $\operatorname{End}(E) \otimes \Omega^{p, q}$ over $\mathcal{U}-D$.

Definition 4.3. We say that $\left(E, \bar{\partial}_{E}, h\right)$ is acceptable at $P$, if the following holds:

- Let $(\mathcal{U}, \varphi)$ be an admissible coordinate around $P$. The norms of the curvature $R(h)$ with respect to the metric $(\cdot, \cdot)_{h, g_{\mathbf{p}}}$ is bounded over $\mathcal{U}-D$.

When $\left(E, \bar{\partial}_{E}, h\right)$ is acceptable at any point $P$, then we say that it is acceptable.

\subsection{Tameness and nilpotentness}

Let $\left(E, \bar{\partial}_{E}, \theta, h\right)$ be a harmonic bundle of rank $r$ defined over $X-D$. 
Definition 4.4. Let $P$ be any point of $X$, and $(\mathcal{U}, \varphi)$ be an admissible coordinate around $P$. On $\mathcal{U}$, we have the description:

$$
\theta=\sum_{j=1}^{l} f_{j} \cdot \frac{d z_{j}}{z_{j}}+\sum_{j=l+1}^{n} g_{j} \cdot d z_{j} .
$$

Tameness. Let $t$ be a formal variable. We have the polynomials $\operatorname{det}(t-$ $\left.f_{j}\right)$ and $\operatorname{det}\left(t-g_{j}\right)$ of $t$, whose coefficients are holomorphic functions defined over $\mathcal{U}-\bigcup_{j=1}^{l} D_{i_{j}}$. When the functions are extended to the holomorphic functions over $\mathcal{U}$, the harmonic bundle is called tame at $P$.

Nilpotentness. Assume that the harmonic bundle is tame at $P$. When $\left.\operatorname{det}\left(t-f_{j}\right)\right|_{\mathcal{U} \cap D_{i_{j}}}=t^{r}$, then the harmonic bundle is called nilpotent at $P$.

When $\left(E, \bar{\partial}_{E}, h, \theta\right)$ is a tame nilpotent at any point $P \in X$, then it is called a tame nilpotent harmonic bundle.

For the tame nilpotent harmonic bundle, we have the following estimate. Note that the proposition is essentially contained in Theorem 1 of [35]. Since we have to care about the dependence of the constant on the family, we give a detailed proof, by following Simpson and Ahlfors $([1])$.

Proposition 4.1 (Simpson). Let $\left(E, \bar{\partial}_{E}, h, \theta\right)$ be a tame nilpotent harmonic bundle. Let $P, f_{j}, g_{j}$ be as above. We put $y_{j}=-\log \left|z_{j}\right|^{2}$ for $j=1, \ldots, l$. Then there exists a positive constant $C>0$ satisfying the following:

$$
\begin{aligned}
& \left|f_{j}\right|_{h} \leq C \cdot y_{j}^{-1}, \quad(j=1, \ldots, l) \\
& \left|g_{j}\right|_{h} \leq C, \quad(j=l+1, \ldots, n) .
\end{aligned}
$$

Proof. From the beginning, we can assume that $\mathcal{U}=\Delta^{n}$, and we can assume that $D=\bigcup_{j=1}^{l} D_{j}$ for $l \leq n$, where we put $D_{j}:=\left\{z_{j}=0\right\}$. We only see that there exists a positive constant $C$ satisfying $\left|f_{j}\right| \leq$ $C \cdot y_{j}^{-1}$ independently of $\left(z_{1}, \ldots, z_{n}\right)$. The estimate for $g_{j}$ can be shown similarly. We can assume that $j=1$. We denote $z_{1}$ by $z$, and we put $f_{0}=z^{-1} \cdot f_{1}$. For any positive number $r$, we put $\psi(r):=(-r$. $\log |r|)^{-1}$. Let $\pi_{1}$ denote the projection $\Delta^{n} \longrightarrow \Delta^{n-1}$, omitting the first component. 
We denote the dual of $f_{0}$ with respect the metric $h$ by $f_{0}^{\dagger}$.

First we note the following:

Lemma 4.1. There exist positive constants $C^{\prime}>0$ and $\epsilon>0$ satisfying the following:

- Let a be one of the eigenvalues of the endomorphism $f_{0}(P)$ of the fiber $E_{\mid P}$. Then the following inequality holds:

$$
|a| \leq C^{\prime} \cdot|z(P)|^{-1+\epsilon}
$$

Proof. Since $\operatorname{det}\left(t-z \cdot f_{0}\right)$ is holomorphic over $\Delta^{n}$, and since $\operatorname{det}(t-$ $\left.z \cdot f_{0}\right)\left.\right|_{z=0}=t^{n}$, the eigenvalues of $\left(z \cdot f_{0}\right)(P)$ is dominated by $C^{\prime} \cdot|z(P)|^{\epsilon}$ for some $C^{\prime}>0$ and $\epsilon>0$. Then we obtain the inequality desired.

q.e.d.

For an element $p \in \Delta^{* n-1}$, the tuple $\left(E_{\mid \pi_{1}^{-1}(P)}, f_{0} \cdot d z, h\right)$ gives a harmonic bundle. Thus we obtain the following inequality due to Simpson (See [34] and [35]):

$$
\Delta \log \left|f_{0}\right|_{h}^{2} \leq-\frac{\left|\left[f_{0}, f_{0}^{\dagger}\right]\right|_{h}^{2}}{\left|f_{0}\right|_{h}^{2}}
$$

Here $\Delta$ is the operator $-\left(\partial_{s}^{2}+\partial_{t}^{2}\right)$ for the real coordinate $z=s+\sqrt{-1} t$. We put $\left|f_{0}\right|_{h}^{2}$.

Lemma 4.2. There are positive numbers $C_{1}$ and $C_{2}$ such that either one of the following holds for any $P \in \Delta^{n}-D$ :

(i) $Q(P) \leq C_{1} \cdot \psi(|z(P)|)^{2}$ or

(ii) $\Delta(\log Q)(P) \leq-C_{2} \cdot Q(P)$.

Proof. Let $P$ be a point of $\Delta^{n}-D$. We take an orthogonal base $\boldsymbol{e}=\left(e_{i}\right)$ of the fiber $E_{\mid P}$ with respect to the metric $h$ such that the filtration $\left\{F_{i}=\left\langle e_{j} \mid j \leq i\right\rangle\right\}$ is preserved by the map $f_{0 \mid P}$. We have the matrix representation $\Gamma$ of $f_{0 \mid P}$ with respect to the base $e$, that is, $f_{0 \mid P} \boldsymbol{e}=\boldsymbol{e} \cdot \Gamma$. Then $\Gamma$ is a triangular matrix by our choice of $\boldsymbol{e}$. We denote the diagonal part of $\Gamma$ by $\Gamma_{0}$ and we put $\Gamma_{1}:=\Gamma-\Gamma_{0}$. Let $\Gamma^{\dagger}$ be the matrix representation of $f_{0}^{\dagger}$ with respect to the base $\boldsymbol{e}$. It is the adjoint matrix of $\Gamma$, that is, $\Gamma^{\dagger}={ }^{t} \bar{\Gamma}$. We denote the diagonal part by $\Gamma_{0}^{\dagger}$ and put $\Gamma_{1}^{\dagger}:=\Gamma^{\dagger}-\Gamma_{0}^{\dagger}$. 
We have the equality $\left[\Gamma, \Gamma^{\dagger}\right]=\left[\Gamma_{0}, \Gamma_{1}^{\dagger}\right]+\left[\Gamma_{1}, \Gamma_{0}^{\dagger}\right]+\left[\Gamma_{1}, \Gamma_{1}^{\dagger}\right]$. We denote the diagonal part of $\left[\Gamma_{1}, \Gamma_{1}^{\dagger}\right]$ by $\Xi$. It is easy to see that there is a positive number $c_{1}$ such that $|\Xi|^{2} \geq c_{1}\left|\Gamma_{1}\right|^{2}$, where $c_{1}$ depends only on the dimension of the vector space $E_{\mid P}$. Note that the diagonal parts of $\left[\Gamma_{0}, \Gamma_{1}^{\dagger}\right]$ and $\left[\Gamma_{1}, \Gamma_{0}^{\dagger}\right]$ are 0 , so that we obtain the inequality $\left|\left[\Gamma, \Gamma^{\dagger}\right]\right| \geq c_{1}\left|\Gamma_{1}\right|^{2}$.

On the other hand, we have $\left|\Gamma_{0}\right| \leq c_{2}|z(P)|^{-1+\epsilon}$ where $c_{2}$ is a positive constant, which follows from the estimates for the eigenvalues. We have the equality $Q=\left|f_{0 \mid P}\right|_{h}^{2}=\left|\Gamma_{0}\right|^{2}+\left|\Gamma_{1}\right|^{2}$. Thus there are positive constants $C_{1}$ and $C_{2}$ such that if $Q \geq C_{1} \cdot \psi(|z(P)|)^{2}$ then $\left|\Gamma_{1}\right|^{4} \geq$ $2^{-1} \cdot Q^{2}$, so $\Delta \log Q \leq-C_{2} \cdot Q$.

q.e.d.

We can assume that $C_{2}=-8$ by taking a multiplication of some constant. Then we know that one of the following holds for any point $P \in \Delta^{* l} \times \Delta^{n-l}:\left(\right.$ i) $Q(P) \cdot|d z \cdot d \bar{z}| \leq C_{1} \cdot \psi(|z(P)|)^{2} \cdot|d z \cdot d \bar{z}|$, or (ii) $\Delta\left(\log Q^{1 / 2}\right)(P) \leq-4 Q(P)$. Note that we can make $C_{1}$ larger. In particular, we can assume that $C_{1}>1$. The (ii) means that the curvature given by the pseudo metric $Q|d z \cdot d \bar{z}|$ is less than -1 .

We use the notation $\Delta_{x}:=\{x \in \Delta\}$ and $\Delta_{z}^{*}:=\left\{z \in \Delta^{*}\right\}$. We take a holomorphic covering map $\Delta_{x} \longrightarrow \Delta_{z}^{*}$. We have the natural isomorphism $\Delta_{z}^{*} \simeq \pi_{1}^{-1}(p)$ for any $p \in \Delta^{* l-1} \times \Delta^{n-l}$. Then we obtain the holomorphic covering map $F: \Delta_{x} \longrightarrow \pi_{1}^{-1}(p)$. The pull back $F^{-1}\left(\psi\left(|z|^{2}\right)|d z d \bar{z}|\right)$ is same as $d \sigma^{2}:=\left(1-|z|^{2}\right)^{-2}|d x d \bar{x}|$, because they are the Poincaré metrics of $\Delta_{x}$. We put $Q_{x}:=F^{-1}(Q) \cdot|d F / d x|$ and $u:=\log Q_{x}^{1 / 2}$. Then one of the following holds for any $P \in \Delta_{x}$ : (i) $Q_{x}(P)|d x d \bar{x}| \leq C_{1} d \sigma^{2}$, or (ii) $\Delta(u)(P) \leq-4 e^{2 u(P)}$.

For any $R<1$ which is sufficiently close to 1 , we put $v_{R}(x):=$ $\log R\left(R^{2}-|x|^{2}\right)^{-1}$ for $|x|<R$. Note that the following equalities:

$$
\Delta v_{R}=-4 e^{2 v_{R}}, \quad d \sigma^{2}=e^{2 v_{1}}|d x d \bar{x}|, \quad v_{1} \leq v_{R} .
$$

We only have to show that $e^{2 u} \leq C_{3} e^{2 v_{1}}$ over $\Delta_{x}$ for some positive constant $C_{3}$, independently of $p \in \Delta^{* l-1} \times \Delta^{n-l}$.

We have already known that there exists a constant $C_{4}>0$ such that one of the following holds for any $P \in \Delta_{x}$ : (i) $u(P) \leq v_{1}(P)+C_{4}$, or (ii) $\Delta(u)(P) \leq-4 e^{2 u(P)}$. We will prove that (i) holds for any $P \in \Delta_{x}$, in fact, in this stage.

We consider the region $S(R):=\left\{P \in \Delta_{x} \mid u(P)>v_{R}(P)+C_{4}\right\}$. On the region, we have the inequality $\Delta\left(u-v_{R}+C_{4}\right) \leq-4\left(e^{2 u}-e^{2 v_{R}}\right)<0$. On $\left\{P \in \Delta_{x}|| x(P) \mid=R\right\}$, we have $v_{R}=\infty$. Thus the boundary of 
$S(R)$ does not intersect with $\left\{P \in \Delta_{x}|| x(P) \mid=R\right\}$. Thus we have the inequality $u-v_{R}+C_{4} \leq 0$ on the boundary of $S(R)$, which raises a contradiction. Thus the region $S(R)$ is empty.

Taking a limit $R \rightarrow 1$, we obtain the desired inequality $u \leq v_{1}+C_{4}$. Thus we have completed the proof of Proposition 4.1. q.e.d.

The following corollary is just a reformulation.

Corollary 4.1. Let $P$ be a point of $X$ and $(\mathcal{U}, \varphi)$ be an admissible coordinate around $P$. We have the metric $(\cdot, \cdot)_{h, g_{\mathbf{p}}}$ of $\operatorname{End}(E) \otimes \Omega_{\mathcal{U}-D}^{p, q}$. The norm of $\theta$ with respect to the metric $(\cdot, \cdot)_{h, g_{\mathbf{p}}}$ is bounded.

Let $\left(E, \bar{\partial}_{E}, \theta, h\right)$ be a tame nilpotent harmonic bundle over $X-D$. Then we have the deformed holomorphic bundle $\left(\mathcal{E}, d^{\prime \prime}, h\right)$ over $\mathcal{X}$, and $\left(\mathcal{E}^{\lambda}, d^{\prime \prime \lambda}, h\right)$ over $\mathcal{X}^{\lambda}$.

Proposition 4.2. The hermitian holomorphic vector bundles $\left(\mathcal{E}^{\lambda}\right.$, $\left.d^{\prime \prime \lambda}, h\right)$ and $\left(\mathcal{E}, d^{\prime \prime}, h\right)$ is acceptable with respect to the divisors $\mathcal{D}^{\lambda}$ and $\mathcal{D}$ respectively.

Proof. The assertion for $\left(\mathcal{E}, d^{\prime \prime}, h\right)$ follows from the formula $(9)$ and Corollary 4.1. Similarly for $\left(\mathcal{E}^{\lambda}, d^{\prime \prime \lambda}, h\right)$.

q.e.d.

\subsection{The tame nilpotent harmonic bundle over the punc- tured disc}

\subsubsection{Prolongation}

We recall some results of Simpson. See the Section 10 of [34] and the Sections 3,4 and 5 of [35]. Let $\left(E, \bar{\partial}_{E}, h\right)$ be a hermitian holomorphic bundle over the punctured disc $\Delta^{*}$. We denote the origin of $\Delta$ by $O$, which gives a smooth divisor of $\Delta$. Simpson showed the following:

Lemma 4.3. Let $\alpha$ be a real number. If $\left(E, \bar{\partial}_{E}, h\right)$ is acceptable, then the prolongation $E_{\alpha}$ is coherent locally free.

Remark 4.1. Our definition of acceptable is slightly different from Simpson's. He showed the stronger result than that stated here. Namely, if the curvature $R(h)$ is dominated by $\left(|z|^{-2}\left(-\log |z|^{2}\right)^{-2}+f\right) d z \cdot d \bar{z}$ for some $L^{p}$-function $f$ on $\Delta$ with respect to the usual measure $|d z \cdot d \bar{z}|$, then prolongments $E_{\alpha}$ are coherent locally free. On the other hand, we assume that $R(h)$ is dominated by $|z|^{-2}\left(-\log |z|^{2}\right)^{-2} d z \cdot d \bar{z}$. We can and will use his stronger result without mention. 
Let $\beta \geq \alpha$ be real numbers. Then we have the naturally defined morphism $E_{\beta} \longrightarrow E_{\alpha}$ of coherent sheaves. We obtain the morphism $E_{\beta \mid O} \longrightarrow E_{\alpha \mid O}$, which gives a descending filtration of the vector space $E_{\alpha \mid O}$. We denote the image of $E_{\beta \mid O}$ by $F^{\beta}\left(E_{\alpha \mid O}\right)$. Similarly we have the morphism $E_{<\beta} \longrightarrow E_{\alpha}$, and thus $E_{<\beta \mid O} \longrightarrow E_{\alpha \mid O}$. We put $G r^{\alpha}=E_{\alpha \mid O} / E_{<\alpha \mid O}$. Then the graduation of the filtration $F^{\cdot}\left(E_{\alpha \mid O}\right)$ is $\bigoplus_{\alpha \leq \beta<\alpha+1} G r^{\beta}$.

Condition 4.1. We mainly consider the case that $\alpha=0$ and $\operatorname{dim}\left(G r^{0}\right)=\operatorname{dim}\left(E_{\mid O}\right)$. In this case, we say that the parabolic structure of $\left(E, \bar{\partial}_{E}, h\right)$ is trivial.

We also refer the following.

Lemma 4.4. Prolongation is compatible with the procedures taking determinant, dual, and tensor products. (See the papers [34] and [35] of Simpson for a precise statement.)

Assume the parabolic structures of all hermitian holomorphic vector bundles are trivial, the the following holds:

- For $\left(E, \bar{\partial}_{E}, h\right)$, we have ${ }^{\diamond} \operatorname{det}(E) \simeq \operatorname{det}\left({ }^{\diamond} E\right)$ and $\left({ }^{\diamond} E\right)^{\vee} \simeq{ }^{\diamond}\left(E^{\vee}\right)$.

- For $\left(E_{i}, \bar{\partial}_{E_{i}}, h_{i}\right)(i=1,2)$, we have ${ }^{\diamond}\left(E_{1} \otimes E_{2}\right)={ }^{\diamond} E_{1} \otimes{ }^{\diamond} E_{2}$. In particular, we have ${ }^{\diamond} \operatorname{Sym}^{l}(E) \simeq \operatorname{Sym}^{l}\left({ }^{\diamond} E\right)$ and ${ }^{\diamond}\left(\bigwedge^{l} E\right) \simeq \bigwedge^{l}\left({ }^{\diamond} E\right)$.

In the case of harmonic bundle, we obtain the following corollary.

Corollary 4.2. Let $\left(E, \bar{\partial}_{E}, h, \theta\right)$ be a tame nilpotent harmonic bundle over $\Delta^{*}$.

- $\mathcal{E}_{\alpha}^{\lambda}$ is coherent locally free for any $\alpha$.

- Let $f$ be a holomorphic section of $\mathcal{E}_{\alpha}^{\lambda}$, and then $\mathbb{D}^{\lambda}(f)$ is a holomorphic section of $\mathcal{E}_{\alpha-1}^{\lambda} \otimes \Omega_{\Delta}^{1,0}$.

Proof. Since $\left(\mathcal{E}^{\lambda}, h\right)$ is acceptable, $\mathcal{E}_{\alpha}^{\lambda}$ is locally free coherent sheaf. By the same argument as that in 737 page of [35], we can obtain the estimate for $d^{\prime \lambda}(f)$ for a holomorphic section $f$ of $\mathcal{E}_{\alpha}^{\lambda}$. Here $d^{\prime \lambda}$ denotes the $(1,0)$-part of of the metric connection of the hermitian holomorphic bundle $\left(\mathcal{E}^{\lambda}, d^{\prime \prime \lambda}, h\right)$. The second claim follows from such estimate. q.e.d.

For each $\lambda$, we obtain the residue $\operatorname{Res}\left(\mathbb{D}^{\lambda}\right)$ :

$$
\operatorname{Res}\left(\mathbb{D}^{\lambda}\right): \mathcal{E}_{\alpha \mid O}^{\lambda} \longrightarrow \mathcal{E}_{\alpha \mid O}^{\lambda}
$$

It preserves the parabolic filtration $F^{\alpha}$. Thus we also obtain the elements of End $\left(G r^{\beta}\right)$, which we denote by $\operatorname{Res}\left(\mathbb{D}^{\lambda}\right)_{\beta}$. 


\section{Lemma 4.5.}

- Let $\left(E, \bar{\partial}_{E}, \theta, h\right)$ be a tame nilpotent harmonic bundle. Assume that the parabolic structure of $\left(\mathcal{E}^{\lambda}, h\right)$ is trivial. Then we have ${ }^{\diamond} \operatorname{det}\left(\mathcal{E}^{\lambda}\right)=\operatorname{det}\left({ }^{\diamond} \mathcal{E}^{\lambda}\right)$, and ${ }^{\diamond}\left(\mathcal{E}^{\lambda \vee}\right)=\left({ }^{\diamond} \mathcal{E}^{\lambda}\right)^{\vee}$. Moreover, the induced residues are same.

- Let $\left(E_{i}, \bar{\partial}_{E_{i}}, \theta_{i}, h_{i}\right)(i=1,2)$ be tame nilpotent harmonic bundles. Assume that the parabolic structure of $\left(\mathcal{E}_{i}^{\lambda}, h_{i}\right)$ are trivial. Then we have ${ }^{\diamond}\left(\mathcal{E}_{1}^{\lambda} \otimes \mathcal{E}_{2}^{\lambda}\right)={ }^{\diamond} \mathcal{E}_{1}^{\lambda} \otimes{ }^{\diamond} \mathcal{E}_{2}^{\lambda}$. The induced residues are isomorphic. In particular, similar things hold for symmetric products and exterior products.

\subsubsection{Some inequalities}

We recall the $\lambda$-connection version of the inequality due to Simpson (Lemma 4.1 of [35]). In this subsubsection, the metric of $\Delta^{*}$ is the standard metric given by $|d z \cdot d \bar{z}|$.

Let $\left(E, d_{E}^{\prime \prime}\right)$ be a holomorphic bundle over $\Delta^{*}$, and $\mathbb{D}^{\lambda}$ be a flat holomorphic $\lambda$-connection on $\left(E, d_{E}^{\prime \prime}\right)$. It is not necessarily obtained from a harmonic metric. Let $h$ be a hermitian metric on $E$, which is not necessarily harmonic. We denote the $(1,0)$-part of the metric connection of $d_{E}^{\prime \prime}$ with respect to $h$ by $d_{E}^{\prime}$. We denote the $(1,0)$-part of $\mathbb{D}^{\lambda}$ by $\mathbb{D}^{\lambda \prime}$, that is $\mathbb{D}^{\lambda}=d_{E}^{\prime \prime}+\mathbb{D}^{\lambda \prime}$.

We put as follows:

$$
\theta:=\frac{1}{1+|\lambda|^{2}}\left(\mathbb{D}^{\lambda \prime}-\lambda d_{E}^{\prime}\right) \in C^{\infty}\left(X, \text { End }(E) \otimes \Omega_{X}^{1,0}\right) .
$$

It is not necessarily holomorphic. Here we start from $\mathbb{D}^{\lambda}$ and the metric. Thus we use the notation $\theta\left(\mathbb{D}^{\lambda}, h\right)$ if we emphasize the dependence of $\theta$ on $\mathbb{D}^{\lambda}$ and $h$.

We denote the adjoint of $\theta$ with respect to $h$ by $\theta^{\dagger}$. Then we put as follows:

$$
\bar{\partial}_{E}:=d_{E}^{\prime \prime}-\lambda \cdot \theta^{\dagger}, \quad \partial_{E}:=d_{E}^{\prime}+\bar{\lambda} \cdot \theta .
$$

Then we put as follows:

$$
G\left(\mathbb{D}^{\lambda}, h\right):=\bar{\partial}_{E}(\theta)
$$

It is easy to see that the tuple $\left(E, \bar{\partial}_{E}, \theta, h\right)$ is harmonic if and only if $G\left(\mathbb{D}^{\lambda}, h\right)=0$.

The following lemma is just a $\lambda$-connection version of Lemma 4.1 of [35]. 
Lemma 4.6. Assume that $\lambda \neq 0$. Let $s$ be a section of $E$ such that $\mathbb{D}^{\lambda}(s)=0$. Then we obtain the following inequality:

$$
\Delta \log |s|^{2} \leq 2\left(|\lambda|^{-1}+|\lambda|\right) \cdot\left|G\left(\mathbb{D}^{\lambda}, h\right)\right|_{h} .
$$

Proof. We denote the curvature of $d_{E}^{\prime}+d_{E}^{\prime \prime}$ by $R\left(h, d_{E}^{\prime \prime}\right)$. By our assumption $\mathbb{D}^{\lambda}(s)=0$, the section $s$ is holomorphic. Thus we have the following equality:

$$
\partial \bar{\partial}|s|_{h}^{2}=\left(d_{E}^{\prime} s, d_{E}^{\prime} s\right)_{h}+\left(s, R\left(h, d_{E}^{\prime \prime}\right) s\right)_{h} .
$$

We also have the following:

$$
R\left(h, d_{E}^{\prime \prime}\right)=\left(\bar{\partial}_{E}+\lambda \cdot \theta^{\dagger}\right) \cdot\left(\partial_{E}-\bar{\lambda} \cdot \theta\right)+\left(\partial_{E}-\bar{\lambda} \cdot \theta\right) \cdot\left(\bar{\partial}_{E}+\lambda \cdot \theta^{\dagger}\right) .
$$

By our assumption of the flatness of $\mathbb{D}^{\lambda}$, we have the following:

$$
0=\mathbb{D}^{\lambda} \circ \mathbb{D}^{\lambda}=\left(\bar{\partial}_{E}+\lambda \cdot \theta^{\dagger}\right) \cdot\left(\lambda \partial_{E}+\theta\right)+\left(\lambda \partial_{E}+\theta\right) \cdot\left(\bar{\partial}_{E}+\lambda \cdot \theta^{\dagger}\right) .
$$

Then we obtain the following by a direct calculation:

$$
R\left(h, d_{E}^{\prime \prime}\right)=-\left(1+|\lambda|^{2}\right) \cdot\left(\theta \wedge \theta^{\dagger}+\theta^{\dagger} \wedge \theta\right)-\lambda^{-1} \cdot\left(1+|\lambda|^{2}\right) \cdot G\left(\mathbb{D}^{\lambda}, h\right) .
$$

By our assumption $\mathbb{D}^{\lambda}(s)=0$, we have $\left(\lambda \partial_{E}+\theta\right) s=0$. Thus we obtain the following:

$$
d_{E}^{\prime}(s)=\left(\partial_{E}-\bar{\lambda} \theta\right) s=-\left(\lambda^{-1}+\bar{\lambda}\right) \cdot \theta(s)=-\lambda^{-1}\left(1+|\lambda|^{2}\right) \cdot \theta(s) .
$$

Hence we obtain the following equality:

$$
\begin{aligned}
\partial \bar{\partial}|s|_{h}^{2}= & \left(|\lambda|^{-2}\left(1+|\lambda|^{2}\right)^{2}-\left(1+|\lambda|^{2}\right)\right) \cdot(\theta s, \theta s)_{h} \\
& -\left(1+|\lambda|^{2}\right) \cdot\left(\theta^{\dagger} s, \theta^{\dagger} s\right)_{h}-\bar{\lambda}^{-1}\left(1+|\lambda|^{2}\right) \cdot\left(s, G\left(\mathbb{D}^{\lambda}, h\right) s\right)_{h} .
\end{aligned}
$$

Then we obtain the following:

$$
\begin{aligned}
\Delta^{\prime \prime}|s|_{h}^{2}= & -\left(1+|\lambda|^{-2}\right) \cdot|\theta s|_{h}^{2}-\left(1+|\lambda|^{2}\right) \cdot\left|\theta^{\dagger} s\right|_{h}^{2} \\
& +\lambda^{-1}\left(1+|\lambda|^{2}\right) \cdot\left(s, \sqrt{-1} \Lambda G\left(\mathbb{D}^{\lambda}, h\right) s\right)_{h} .
\end{aligned}
$$

Here $\Lambda$ denotes the operator from $\Omega^{1,1}$ to $\mathbf{C}$, such that $d z \cdot d \bar{z} \longmapsto-\sqrt{-1}$. 
We have the following equality:

$$
\Delta^{\prime \prime} \log |s|_{h}^{2}=\frac{\Delta^{\prime \prime}|s|_{h}^{2}}{|s|_{h}^{2}}-\frac{\left|\left(s, d_{E}^{\prime} s\right)_{h}\right|^{2}}{|s|_{h}^{4}} .
$$

Due to (30), we have the following:

$$
\left|\left(s, d_{E}^{\prime} s\right)_{h}\right|^{2}=\left(|\lambda|^{-2}+1\right) \cdot\left|(s, \theta s)_{h}\right|^{2}+\left(1+|\lambda|^{2}\right) \cdot\left|\left(s, \theta^{\dagger} s\right)_{h}\right|^{2} .
$$

Thus we obtain the inequality desired.

q.e.d.

Assume that $|\theta|_{h}$ is dominated by $|z|^{-2}\left(-\log |z|^{2}\right)^{2}$. Then, due to the equality (29), the prolongments $E_{\alpha}$ are locally free (See Remark 4.1). When we emphasize the dependence of the prolongments $E_{\alpha}$ to the metric $h$, we use the notation $E_{\alpha}(h)$ instead of $E_{\alpha}$.

Corollary 4.3. Assume that $\lambda \neq 0$. Let $\left(E, \mathbb{D}^{\lambda}\right)$ be as above. Let $h_{1}$ and $h_{2}$ satisfy the following conditions:

1. For $i=1,2$, the hermitian bundles $\left(E, h_{i}\right)$ are acceptable. and the functions $\left|G\left(\mathbb{D}^{\lambda}, h_{i}\right)\right|_{h_{i}}$ are $L^{p}$.

2. We have $E_{\alpha}\left(h_{1}\right)=E_{\alpha}\left(h_{2}\right)$ and $E_{\alpha}^{\vee}\left(h_{1}\right)=E_{\alpha}^{\vee}\left(h_{2}\right)$ for any $\alpha$. Note that it also implies the coincidence of the parabolic structures.

Then the metrics $h_{1}$ and $h_{2}$ are mutually bounded. [35].

Proof. The argument is same as Corollary 4.2 and Corollary 4.3 of q.e.d.

\subsubsection{Norm estimate in one dimensional case}

In this subsubsection, we give a norm estimate in one dimensional case. In [35], Simpson discussed the cases $\lambda=0$ and $\lambda=1$. Clearly his argument works in general case. We only have to indicate how to change.

We recall the argument of Theorem 4 of [35]. Let $\left(E, \bar{\partial}_{E}, \theta, h\right)$ be a tame harmonic bundle over a punctured disc. We have the $\lambda$-connection $\left(\mathcal{E}^{\lambda}, \mathbb{D}^{\lambda}\right)$. Pick a real number $\alpha \in \mathbf{R}$. Then we have the residues of $\mathbb{D}^{\lambda}$ :

$$
\left(G r^{\beta}, \operatorname{Res}\left(\mathbb{D}^{\lambda}\right)_{\beta}\right) \quad(\alpha \leq \beta<\alpha+1) .
$$

We decompose $G r^{\beta}$ into the generalized eigenspaces of $\operatorname{Res}\left(\mathbb{D}^{\lambda}\right)_{\beta}$.

$$
\left(G r^{\beta}, \operatorname{Res}\left(\mathbb{D}^{\lambda}\right)_{\beta}\right)=\bigoplus_{\omega}\left(G r_{\omega}^{\beta}, \operatorname{Res}\left(\mathbb{D}^{\lambda}\right)_{\beta, \omega}\right)
$$


Here $\omega$ runs through the set of eigenvalues of $\operatorname{Res}\left(\mathbb{D}^{\lambda}\right)_{\beta}$. The pair $\left(G r_{\omega}^{\beta}, \operatorname{Res}\left(\mathbb{D}^{\lambda}\right)_{\beta, \omega}\right)$ of the vector space and the endomorphism is called the $(\beta, \omega)$-part of $\left(\mathcal{E}_{\alpha}^{\lambda}, \mathbb{D}^{\lambda}, h\right)$.

For $(\beta, \omega)$, we denote the nilpotent part of $\operatorname{Res}\left(\mathbb{D}^{\lambda}\right)_{\beta, \omega}$ by $N(\beta, \omega)$, and we put $V(\beta, \omega):=G r_{\omega}^{\beta}$.

Let us consider the following harmonic bundle (See Subsection 3.2):

$$
\left(E_{0}, \bar{\partial}_{E_{0}}, \theta_{0}, h_{0}\right):=\bigoplus_{(\beta, \omega)} E(V(\beta, \omega), N(\beta, \omega)) \otimes L\left(C_{1}, C_{2}\right)
$$

Here $C_{1}$ and $C_{2}$ are real numbers depending on $\beta$ and $\omega$, given as follows:

$$
C_{1}=\frac{\left(1-|\lambda|^{2}\right) \cdot \beta+2 \operatorname{Re}(\bar{\lambda} \cdot \omega)}{1+|\lambda|^{2}}, \quad C_{2}=\frac{\beta-\lambda \cdot \omega}{1+|\lambda|^{2}} .
$$

We have the corresponding deformed holomorphic bundle $\mathcal{E}_{0}^{\lambda}$ on $\mathcal{X}^{\lambda}$, and the $\lambda$-connection $\mathbb{D}_{0}^{\lambda}$. By our construction, the $(\beta, \omega)$-part of $\left(\mathcal{E}_{0, \alpha}^{\lambda}, \mathbb{D}_{0}^{\lambda}\right.$, $\left.h_{0}\right)$ is isomorphic to the $(\beta, \omega)$-part of $\left(\mathcal{E}_{\alpha}^{\lambda}, \mathbb{D}^{\lambda}, h\right)$.

Lemma 4.7. There exists a holomorphic isomorphism $f: \mathcal{E}_{0}^{\lambda} \longrightarrow$ $\mathcal{E}^{\lambda}$ satisfying the following:

1. We put $g_{1}:=f_{\mid O} \circ \operatorname{Res}\left(\mathbb{D}_{0}^{\lambda}\right)-\operatorname{Res}\left(\mathbb{D}^{\lambda}\right) \circ f_{\mid O}$ and $g_{2}:=f_{\mid O}^{-1} \circ$ $\operatorname{Res}\left(\mathbb{D}^{\lambda}\right)-\operatorname{Res}\left(\mathbb{D}_{0}^{\lambda}\right) \circ f_{\mid O}^{-1}$. Then $g_{1}\left(F^{\beta}\right) \subset F^{<\beta}$ and $g_{2}\left(F^{\beta}\right) \subset F^{<\beta}$.

Proof. First of all, we take an isomorphism $f_{\mid O}: \mathcal{E}_{0, \alpha \mid O}^{\lambda} \longrightarrow \mathcal{E}_{\alpha \mid O}^{\lambda}$ such that Condition 1 holds. It is possible because the graded parts of the endomorphisms $\operatorname{Res}\left(\mathbb{D}^{\lambda}\right)$ and $\operatorname{Res}\left(\mathbb{D}_{0}^{\lambda}\right)$ are isomorphic. And then we only have to extend $f_{\mid O}$ to a holomorphic map $f$ over $\Delta$. q.e.d.

By the isomorphism $f$, we identify $\mathcal{E}^{\lambda}$ and $\mathcal{E}_{0}^{\lambda}$. Thus the metric $h_{0}$ and the $\lambda$-connection $\mathbb{D}_{0}^{\lambda}$ induces the metric and the $\lambda$-connection on $\mathcal{E}^{\lambda}$. Let us compare $h$ and $h_{0}$. It is clear by our construction that Condition 2 in Corollary 4.3 is satisfied for $h$ and $h_{0}$. Recall that we obtain the non-holomorphic Higgs field from the $\lambda$-connection and the metric (See (28)). Here we have the following:

$$
\theta\left(h, \mathbb{D}^{\lambda}\right)=\theta, \quad \theta\left(h_{0}, \mathbb{D}_{0}^{\lambda}\right)=\theta_{0} .
$$

They are not same in general. We also have $\theta_{1}=\theta\left(h_{0}, \mathbb{D}^{\lambda}\right)$, which is not same as both of them above. 
Lemma 4.8. Condition 1 in Corollary 4.3 is satisfied for the metrics $h$ and $h_{0}$ and the $\lambda$-connection $\mathbb{D}^{\lambda}$.

Proof. Precisely we have to show the following:

- $\left|G\left(h, \mathbb{D}^{\lambda}\right)\right|_{h}$ is $L^{p}$.

- $\left|G\left(h_{0}, \mathbb{D}^{\lambda}\right)\right|_{h_{0}}$ is $L^{p}$.

Since $\left(E, \bar{\partial}_{E}, \theta, h\right)$ is harmonic, we know $G\left(\mathbb{D}^{\lambda}, h\right)=\bar{\partial}_{E}(\theta)=0$. Thus we only have to care $\theta_{1}=\theta\left(h_{0}, \mathbb{D}^{\lambda}\right)$ and $G\left(h_{0}, \mathbb{D}^{\lambda}\right)$. Let $\theta_{1}^{\dagger}$ denote the conjugate of $\theta_{1}$ with respect to $h_{0}$.

Let $\delta^{\prime}$ denote the $(1,0)$-part of the metric connection of $\mathcal{E}^{\lambda}$ with respect to the metric $h_{0}$. By definition, we have the following:

$$
\theta_{1}-\theta_{0}=\frac{1}{|\lambda|^{2}+1}\left(\mathbb{D}^{\lambda}-\lambda \cdot \delta^{\prime}-\mathbb{D}_{0}^{\lambda}+\lambda \cdot \delta^{\prime}\right)=\frac{1}{|\lambda|^{2}+1}\left(\mathbb{D}^{\lambda}-\mathbb{D}_{0}^{\lambda}\right) .
$$

We only have to recall the argument in page 747 of [35]: We put $A=$ $\mathbb{D}^{\lambda}-\mathbb{D}_{0}^{\lambda} \in \Gamma\left(\Delta^{*}, \operatorname{End}\left(\mathcal{E}^{\lambda}\right)\right)$. By our choice of $f$, the order of $A$ is less than $-1+\epsilon$ for some positive $\epsilon>0$. By (29), we have the following:

$$
\begin{aligned}
& \lambda^{-1} \cdot\left(1+|\lambda|^{2}\right) \cdot G\left(\mathbb{D}^{\lambda}, h_{0}\right) \\
& =-\left(1+|\lambda|^{2}\right) \cdot\left(\theta_{1} \wedge \theta_{1}^{\dagger}+\theta_{1}^{\dagger} \wedge \theta_{1}\right)-R\left(h_{0}, d_{\mathcal{E}_{0}^{\lambda}}^{\prime \prime}\right) .
\end{aligned}
$$

Since $h_{0}$ and $\mathbb{D}_{0}^{\lambda}$ is obtained from harmonic bundles, we have the following:

$$
\begin{aligned}
0 & =\lambda^{-1} \cdot\left(1+|\lambda|^{2}\right) \cdot G\left(\mathbb{D}_{0}^{\lambda}, h_{0}\right) \\
& =-\left(1+|\lambda|^{2}\right) \cdot\left(\theta_{0} \wedge \theta_{0}^{\dagger}+\theta_{0}^{\dagger} \wedge \theta_{0}\right)-R\left(h_{0}, d_{\mathcal{E}_{0}^{\lambda}}^{\prime \prime}\right)
\end{aligned}
$$

Hence we obtain the following:

$$
\begin{aligned}
\lambda^{-1} G\left(\mathbb{D}^{\lambda}, h_{0}\right) & =\left(\theta_{0} \wedge \theta_{0}^{\dagger}+\theta_{0}^{\dagger} \wedge \theta_{0}-\theta_{1} \wedge \theta_{1}^{\dagger}+\theta_{1}^{\dagger} \wedge \theta_{1}\right) \\
& =\frac{-1}{|\lambda|^{2}+1}\left(\left[A, A^{\dagger}\right]+\left[A, \theta^{\dagger}\right]+\left[\theta, A^{\dagger}\right]\right) .
\end{aligned}
$$

Hence we obtain the estimate $G\left(\mathbb{D}^{\lambda}, h_{0}\right) \leq C \cdot|z|^{-2+\epsilon}$ for some $\epsilon>0$. Hence $\left|G\left(\mathbb{D}^{\lambda}, h_{0}\right)\right|_{h_{0}}$ is $L_{p}$ with respect to the measure $|d z \cdot d \bar{z}|$. q.e.d.

We have a direct corollary.

Corollary 4.4. The metrics $h$ and $h_{0}$ above are mutually bounded. 
We can reword Corollary 4.4. The nilpotent part of $\operatorname{Res}\left(\mathbb{D}^{\lambda}\right)_{(\beta, \omega)}$ induces the weight filtration $W_{(\beta, \omega)}$ on $G r_{\omega}^{\beta}$. The filtrations $\left\{W_{(\beta, \omega)} \mid \omega\right.$ eigenvalue $\}$ give the filtration of $G r^{\beta}=\bigoplus_{\omega} G r_{\omega}^{\beta}$. Let $W$ denote the filtration of $\bigoplus_{\beta} G r^{\beta}$. We put $F_{\beta}=F^{-\beta} \subset \mathcal{E}_{\alpha \mid O}^{\lambda}$. Then $F_{\beta}$ gives an ascending filtration. Then we obtain the sequence of the filtrations $(F ., W$.$) . Take a holomorphic frame \boldsymbol{v}=\left(v_{1}, \ldots, v_{r}\right)$ of $\mathcal{E}_{\alpha}^{\lambda}$ over $\Delta$ satisfying the following:

- $\boldsymbol{v}_{\mid O}$ is compatible with the ascending filtration $\left\{F_{\beta}\right\}$.

- The induced base $\boldsymbol{v}^{(1)}$ of $\bigoplus G r_{\beta}$ is compatible with the filtration $W$.

Such frame $\boldsymbol{v}$ is called compatible with $\left(F ., W\right.$.). The element $v_{i \mid O}$ induces an element of $\bigoplus G r_{\beta}$, which we denote by $v_{i}^{(1)}$. We put as follows:

$$
\alpha_{i}=\operatorname{deg}^{F \cdot}\left(v_{i \mid O}\right), \quad k_{i}=\frac{1}{2} \operatorname{deg}^{W}\left(v_{i}^{(1)}\right) .
$$

We have the $C^{\infty}$-frame $\boldsymbol{v}^{\prime}=\left(v_{1}^{\prime}, \ldots, v_{r}^{\prime}\right)$ of $\mathcal{E}^{\lambda}$ over $\Delta^{*}$, defined as follows:

$$
v_{i}^{\prime}=|z|^{\alpha_{i}} \cdot(-\log |z|)^{-k_{i}} \cdot v_{i} .
$$

Corollary 4.5. The frame $\boldsymbol{v}^{\prime}$ is adapted (See Definition 2.15).

Proof. The claim follows from Corollary 3.5 and Corollary 4.4. q.e.d.

Let $f$ be a holomorphic section of $\mathcal{E}_{\alpha}^{\lambda}$ over $\Delta$. We have the number $\alpha(f):=\operatorname{deg}^{F}(f(O))$ and $k(f):=2^{-1} \operatorname{deg}^{W}\left(f^{(1)}(O)\right)$. Here $F$ is the ascending filtration above.

Corollary 4.6. There exists positive constants $C_{1}$ and $C_{2}$ such that the following holds over $\Delta^{*}$ :

$$
0<C_{1}<(-\log |z|)^{-k(f)} \cdot|z|^{\alpha(f)} \cdot|f|_{h}<C_{2} .
$$

\subsubsection{Finiteness of some norms and some consequences}

In this section, the metric of $\Delta^{*}$ is the standard one given by $|d z \cdot d \bar{z}|$. Let $\left(E, \bar{\partial}_{E}, \theta, h\right)$ be a tame nilpotent harmonic bundle over $\Delta^{*}$. We have a prolongment ${ }^{\diamond} E$ by increasing order 0 . Then we have the vector spaces. We take a model bundle $\left(E_{0}, \theta_{0}, h_{0}\right)$ and $f:{ }^{\diamond} E_{0} \longrightarrow{ }^{\diamond} E$ as in 
Lemma 4.7. We will identify $E_{0}$ and $E$ over $\Delta^{*}$ by the morphism $f$. We have already known that the metrics $h$ and $h_{0}$ are mutually bounded.

We have the deformed holomorphic bundle $\left(\mathcal{E}^{\lambda}, \mathbb{D}^{\lambda}, h\right)$ and $\left(\mathcal{E}_{0}^{\lambda}, \mathbb{D}_{0}^{\lambda}\right.$, $\left.h_{0}\right)$ over $\{\lambda\} \times \Delta^{*}$, which are induced by $(E, \theta, h)$ and $\left(E_{0}, h_{0}, \theta_{0}\right)$ respectively.

We have the parabolic filtration and the weight filtration for ${ }^{\diamond} \mathcal{E}^{\lambda}$ and ${ }^{\diamond} \mathcal{E}_{0}^{\lambda}$. We denote them by $(F, W)$ and $\left(F_{0}, W_{0}\right)$ respectively.

Take a holomorphic frame $\boldsymbol{v}$ of ${ }^{\diamond} \mathcal{E}^{\lambda}$ such that it is compatible with the sequence of the filtrations $(F, W)$. We put as follows:

$$
\alpha\left(v_{i}\right):=\operatorname{deg}^{F}\left(v_{i}(O)\right), \quad k\left(v_{i}\right):=\frac{1}{2} \operatorname{deg}^{W}\left(v_{i}^{(1)}\right) .
$$

Here $v_{i}^{(1)}$ denotes the induced element of $G r^{F}$. We also take a holomorphic frame $\boldsymbol{v}_{0}$ of ${ }^{\diamond} \mathcal{E}_{0}^{\lambda}$ such that it is compatible with the sequence of the filtrations $\left(F_{0}, W_{0}\right)$. Similarly we obtain the numbers $\alpha\left(v_{0, i}\right)$ and $k\left(v_{0, i}\right)$.

Since the underlying $C^{\infty}$-vector bundles of $\mathcal{E}^{\lambda}$ and $\mathcal{E}_{0}^{\lambda}$ are naturally identified with $E$ over $\Delta^{*}, \boldsymbol{v}$ and $\boldsymbol{v}_{0}$ give the $C^{\infty}$-frames of $E$ over $\Delta^{*}$. Let $\mathcal{I}$ denote the $C^{\infty}$-isomorphism. Then we have the functions $I_{i j} \in C^{\infty}\left(\Delta^{*}, \mathbf{C}\right)$ determined as follows:

$$
\mathcal{I}\left(v_{0 j}\right)=\sum_{i} I_{i j} \cdot v_{i}
$$

Remark 4.2. Note that our rule of subscription is different from the rule in [35]. There, our $I_{i j}$ is denoted by $I_{j i}$ in Section 7 of [35]. We also note that the choice of the signature of $\alpha\left(v_{i}\right)$ is opposite to that of Simpson.

Simpson considered the following norms $\|\cdot\|_{Z}$ and $\|\cdot\|_{W}$ for a function $f$ on $\Delta^{*}(C)$ :

$$
\begin{aligned}
\|f\|_{Z, C} & =\int_{\Delta^{*}(C)}|f| \cdot \frac{d r \cdot d \alpha}{r \cdot(-\log r)}, \\
\|f\|_{W, C} & =\int_{\Delta^{*}(C)}|f| \cdot \frac{d r \cdot d \alpha}{r \cdot(-\log r) \cdot \log (-\log r)} .
\end{aligned}
$$

Here the real coordinate $z=r \cdot \exp (2 \pi \sqrt{-1} \alpha)$ is used. Simpson showed the following lemma to show that the conjugacy classes of the resides are invariant (See Section 7 in [35]). 
Lemma 4.9. For any $i$ and $j$, we have the following finiteness:

$$
\left\|\bar{z} \cdot \bar{\partial}_{z} I_{i j \mid \Delta^{*}(C)} \cdot|\log r|^{k\left(v_{i}\right)-k\left(v_{0, j}\right)+1} \cdot r^{-\alpha\left(v_{i}\right)+\alpha\left(v_{0, j}\right)}\right\|_{Z, C}<\infty .
$$

For any $i$ and $j$ such that $\alpha\left(v_{i}\right)-\alpha\left(v_{0, j}\right) \neq 0$, there exists a positive constant $C>0$ satisfying the following:

$$
\left|I_{i j}\right| \cdot(-\log r)^{k\left(v_{i}\right)-k\left(v_{0, j}\right)} \cdot r^{-\alpha\left(v_{i}\right)+\alpha\left(v_{0, j}\right)} \leq C(-\log |z|)^{-1} .
$$

For any $i$ and $j$ such that $\alpha\left(v_{i}\right)-\alpha\left(v_{0, j}\right)=0$ and that $k\left(v_{i}\right)-k\left(v_{j}^{0}\right) \neq 0$, we have the following finiteness:

$$
\left\|\left.I_{i j}\right|_{\{\lambda\} \times B^{*}(C)}|\log r|^{\left(k\left(v_{i}\right)-k\left(v_{0, j}\right)\right)}\right\|_{W, C}<\infty .
$$

Proof. We have $\bar{\partial}_{z}(\mathcal{I})=\lambda \cdot\left(\theta^{\dagger}-\theta_{0}^{\dagger}\right)$ by definition of the holomorphic structures of $\mathcal{E}_{0}^{\lambda}$ and $\mathcal{E}^{\lambda}$. Here $\theta_{0}^{\dagger}$ denotes the adjoint of $\theta_{0}$ with respect to the metric $h_{0}$. Simpson showed the following inequality (Lemma 7.7 in $[35])$ :

$$
\int\left|\theta^{\dagger}-\theta_{0}^{\dagger}\right|_{h} \cdot(-\log |z|) \cdot|d z \cdot d \bar{z}|<\infty
$$

Here the metric $h$ is used. Since $h_{0}$ and $h$ are mutually bounded, we can also use $h_{0}$. In fact, we can take any metric mutually bounded to $h_{0}$ and $h$. Thus we do not have to care a choice of the metric in the following.

Recall that the frames $\boldsymbol{v}^{\prime}=\left(v_{i}\right)$ and $\boldsymbol{v}_{0}^{\prime}=\left(v_{0, i}^{\prime}\right)$ are adapted, if we put as follows:

$$
\begin{aligned}
v_{i}^{\prime} & :=|z|^{\alpha\left(v_{i}\right)} \cdot(-\log |z|)^{-k\left(v_{i}\right)} \cdot v_{i} \\
v_{0, j}^{\prime} & :=|z|^{\alpha\left(v_{0, j}\right)} \cdot(-\log |z|)^{-k\left(v_{0, j}\right)} \cdot v_{0, j} .
\end{aligned}
$$

Since we know that $\left|\theta^{\dagger}\right|_{h}$ and $\left|\theta_{0}^{\dagger}\right|_{h}$ are bounded by $(-|z| \cdot \log |z|)^{-1}$, we have the inequality:

$$
\begin{aligned}
& \left.\left|\bar{\partial} I_{i j} \cdot\right| z\right|^{-\alpha\left(v_{i}\right)+\alpha\left(v_{0, j}\right)} \cdot(-\log |z|)^{k\left(v_{i}\right)-k\left(v_{0, j}\right)} \mid \\
& \quad \leq C \cdot|\lambda| \times(-|z| \cdot \log |z|)^{-1} .
\end{aligned}
$$

Thus any components $\bar{\partial}_{z} I_{i j}$ satisfy the inequality (32).

We obtain the inequalities (33) and (34) by using Lemma 7.1, Lemma 7.8 and the argument of Corollary 7.10 in [35]. $\quad$ q.e.d.

Note that many of the arguments in Section 7 in [35] are not needed in our case, for we assumed that the residue of $\left(E, \bar{\partial}_{E}, \theta, h\right)$ are nilpotent. 
Corollary 4.7. The conjugacy classes of the residues $\left(G r_{\omega}^{\beta}\right.$, $\left.\operatorname{Res}\left(\mathbb{D}^{\lambda}\right)_{(\beta, \omega)}\right)$ are independent of $\lambda$.

Proof. Simpson showed that the conjugacy classes of $\operatorname{Res}\left(\mathbb{D}^{\lambda}\right)_{\mid(1, O)}$ and $\operatorname{Res}\left(\mathbb{D}^{\lambda}\right)_{\mid(0, O)}$ are same in [35] by using the inequality (34). By the same method and Lemma 4.9 , we can show that the conjugacy classes of $\operatorname{Res}\left(\mathbb{D}^{\lambda}\right)_{(\beta, \omega) \mid(\lambda, O)}$ and $\operatorname{Res}\left(\mathbb{D}^{0}\right)_{(\beta, \omega) \mid(0, O)}$ are same for any $\lambda \neq 0$. q.e.d.

In particular, we obtain the following.

Corollary 4.8. Let $(E, \theta, h)$ be a tame nilpotent harmonic bundle over $\Delta^{*}$. We also assume that the parabolic structure of the prolongment ${ }^{\diamond} \mathrm{E}$ is trivial. Then the following holds:

- The parabolic structure of the prolongment ${ }^{\diamond} \mathcal{E}^{\lambda}$ is trivial for each $\lambda$.

- All of the eigenvalues of $\operatorname{Res}\left(\mathbb{D}^{\lambda}\right)$ on ${ }^{\diamond} \mathcal{E}_{\mid O}^{\lambda}$ are 0 .

- The conjugacy classes of $\left({ }^{\diamond} \mathcal{E}^{\lambda}{ }_{\mid O}, \operatorname{Res}\left(\mathbb{D}^{\lambda}\right)\right)$ are independent of $\lambda$.

Remark 4.3. Even if $\left(E, \bar{\partial}_{E}, \theta, h\right)$ is tame nilpotent with trivial parabolic structure, it is not clear, a priori, that $\left(\mathcal{E}^{\lambda}, \mathbb{D}^{\lambda}\right)$ are so. That is one of the reasons why we considered the general tame harmonic bundles over the punctured disc.

Let $\left(E, \bar{\partial}_{E}, \theta, h\right)$ be a tame nilpotent harmonic bundle over $\Delta^{*}$. Assume that the parabolic structure of ${ }^{\diamond} E$ is trivial. When $\lambda \neq 0$, we have the flat holomorphic bundle $\left(\mathcal{E}^{\lambda}, \mathbb{D}^{\lambda, f}\right)$. We have a holomorphic frame $\boldsymbol{w}$ of $\mathcal{E}^{\lambda}$ over $\Delta^{*}$ satisfying the following:

- We have the flat connection form $A \in \Gamma\left(\Delta, M(r) \otimes \Omega_{\Delta}(\log O)\right)$ determined by the relation $\mathbb{D}^{\lambda, f} \boldsymbol{w}=\boldsymbol{w} \cdot A$. Then $A$ is of the form $A_{0} d z / z$ for some constant matrix $A_{0}$.

- All of the eigenvalues of $A_{0}$ are 0 .

Such frame is called a normalizing frame in this paper. We have the prolongment of $\mathcal{E}^{\lambda}$ by a normalizing frame.

Lemma 4.10. The prolongment by a normalizing frame is same as the prolongment ${ }^{\diamond} \mathcal{E}^{\lambda}$ by an increasing order 0 .

In particular, a normalizing frame naturally gives a frame of the prolongation ${ }^{\diamond} \mathcal{E}^{\lambda}$. 
Proof. The claim follows from the uniqueness of the prolongation, for which the holomorphic connection is of log type. (See [12]. In particular, II Section 5.)

q.e.d.

\subsection{Definition of trivial parabolic structure}

Let $X$ be an $n$-dimensional complex manifold, and $D$ be a normal crossing divisor of $X$. Let $\left(E, \bar{\partial}_{E}, h\right)$ be a hermitian holomorphic bundle over $X-D$. Let $C$ be a curve contained in $X$, transversal with $D$. Then we obtain the hermitian holomorphic bundle $\left(E, \bar{\partial}_{E}, h\right)_{\mid C-C \cap D}$.

Definition 4.5. We say that the parabolic structure of the hermitian holomorphic bundle $\left(E, \bar{\partial}_{E}, h\right)$ over $X-D$ is trivial, if the following holds:

For any curve $C \subset X$ transversal with $D$, the hermitian holomorphic bundle $\left(E, \bar{\partial}_{E}, h\right)_{\mid C-C \cap D}$ over $C-C \cap D$ is trivial in the sense of Condition 4.1.

As an example, consider the case that $X=\Delta^{n}$ and $D=\bigcup_{j=1}^{l} D_{j}$, where $D_{j}=\left\{z_{j}=0\right\}$. The projection $\Delta^{* l} \times \Delta^{n-l} \longrightarrow \Delta^{* l-1} \times \Delta^{n-l}$, omitting the $j$-th component, is denoted by $\pi_{j}$. For any element $a \in$ $\Delta^{* l-1} \times \Delta^{n-l}$, we obtain the curve $\pi_{j}^{-1}(a) \subset \Delta^{* l} \times \Delta^{n-l}$. Let $\left(E, \bar{\partial}_{E}, h\right)$ be a hermitian holomorphic bundle over $X-D=\Delta^{* l} \times \Delta^{n-l}$. Then the parabolic structure is trivial if and only if the parabolic structure of $\left(E, \bar{\partial}_{E}, h\right)_{\mid \pi_{j}^{-1}(a)}$ is trivial for any $a \in \Delta^{* l-1} \times \Delta^{n-l}$ and $j=1, \ldots, l$.

Corollary 4.9. Let $\left(E, \bar{\partial}_{E}, \theta, h\right)$ be a tame nilpotent harmonic bundle over $X-D$. Assume that the parabolic structure of the hermitian holomorphic bundle $\left(E, \bar{\partial}_{E}, h\right)$ is trivial.

- The parabolic structure of $\left(\mathcal{E}^{\lambda}, d^{\prime \prime \lambda}, h\right)$ is trivial for any $\lambda$.

- All of the eigenvalues of $\operatorname{Res}\left(\mathbb{D}^{\lambda}\right)$ are 0 for any $\lambda$.

- If $\lambda \neq 0$, then we have the flat holomorphic bundle $\left(\mathcal{E}^{\lambda}, \mathbb{D}^{\lambda}\right)$. All of the eigenvalues of the monodromies around the divisor $D$ are 1 .

Proof. The claims follow immediately from Corollary 4.8. $\quad$ q.e.d. 


\subsubsection{Rank 1}

Consider the easy case, that is, the rank 1 case:

Lemma 4.11. Let $\left(E, \bar{\partial}_{E}, \theta, h\right)$ be a tame nilpotent harmonic bundle of rank 1 over $\Delta^{* l} \times \Delta^{n-l}$. Assume that the parabolic structure is trivial. Then it is naturally extended to the harmonic bundle over $\Delta^{n}$. The deformed holomorphic bundle is also extended to that over $\mathbf{C}_{\lambda} \times \Delta^{n}$.

Proof. Consider the holomorphic bundle $\left(E, \bar{\partial}_{E}+\theta^{\dagger}\right)$ with the flat connection $\mathbb{D}^{1}=\bar{\partial}_{E}+\partial_{E}+\theta^{\dagger}+\theta$. Since the eigenvalue of the monodromy is 1 , we can take a holomorphic frame $e$ of $E$ over $X-D$ satisfying the following:

$$
\mathbb{D}^{1}(e)=\left(\bar{\partial}_{E}+\theta^{\dagger}\right) e=\left(\partial_{E}+\theta\right) e=0
$$

The (1,0)-part of the metric connection of $\bar{\partial}_{E}+\theta^{\dagger}$ with respect to $h$ is given by $\partial_{E}-\theta$. We put $h_{0}=h(e, e) \in C^{\infty}(X-D, \mathbf{R})$. Then we have the following equation:

$$
\left(\partial h_{0}\right) \cdot h_{0}^{-1}=\partial\left(\log h_{0}\right)=-2 \theta .
$$

Since the rank of $E$ is 1 , the sheaf $\operatorname{End}(E)$ is naturally isomorphic to $\mathcal{O}$. The tameness and the nilpotentness of $\theta$ implies that $\theta$ is, in fact, a holomorphic section of $\Omega_{\Delta^{n}}^{1,0}$. We also have the equality $\partial(\theta)=0$, because $\left(E, \bar{\partial}_{E}, \theta, h\right)$ is harmonic. Thus we have a holomorphic function $f$ such that $\partial(f)=\theta$.

We have the following equality:

$$
\partial\left(\log \left(h_{0}\right)+2 f\right)=0 .
$$

Note that $\log \left(h_{0}\right)$ is $\mathbf{R}$-valued. Thus we can conclude that $\log \left(h_{0}\right)=$ $-4 \operatorname{Re}(f)+C$ for some constant $C \in \mathbf{R}$, that is, we obtain the following:

$$
h_{0}=\exp (-4 R e(f)+C) .
$$

It means that the increasing order of $e$ is 0 , i.e., $e$ is a holomorphic frame of $\diamond \mathcal{E}^{1}$, and $h$ induces the $C^{\infty}$-metric of ${ }^{\diamond} \mathcal{E}^{1}$.

We put $v:=\exp ((1-\lambda) \bar{f}) \cdot e$. We have the equality $\theta^{\dagger}=\bar{\partial}(\bar{f})$. Then we obtain the following equality:

$$
\begin{aligned}
\left(\bar{\partial}_{E}+\lambda \theta^{\dagger}\right) \cdot v & =\left(\bar{\partial}_{E}+\bar{\partial}(\bar{f})\right) \cdot(\exp ((1-\lambda) \bar{f}) \cdot e)+(\lambda-1) \cdot \bar{\partial}(\bar{f}) \cdot v \\
& =0 .
\end{aligned}
$$


We have $h(v, v)=\exp (-2 \operatorname{Re}((1+\lambda) \bar{f}))$. Thus $v$ gives a holomorphic frame of $\diamond \mathcal{E}$ over $\mathbf{C}_{\lambda} \times \Delta$.

q.e.d.

Corollary 4.10. Let $X$ be a complex manifold, and $D$ be a normal crossing divisor. Let $\left(E, \bar{\partial}_{E}, \theta, h\right)$ be a rank 1 tame nilpotent harmonic bundle with trivial parabolic structure over $X-D$. Then it is naturally extended to the harmonic bundle over $X$. The deformed holomorphic bundle $(\mathcal{E}, h)$ over $\mathcal{X}-\mathcal{D}$ is extended to that over $\mathcal{X}$.

\subsection{Some preliminary}

We recall some tools and ideas from [11], which will be used in the proof of the local freeness of ${ }^{\diamond} \mathcal{E}$.

\subsubsection{Some results of Andreotti-Vesentini}

We recall some results of Andreotti-Vesentini in [2]. Let $(Y, g)$ be a complete Kahler manifold, not necessarily compact. We denote the natural volume form by dvol. Let $\left(E, \bar{\partial}_{E}, h\right)$ be a hermitian holomorphic bundle over $Y$. The hermitian metric $h$ and the Kahler metric $g$ induce the fiberwise hermitian metric of $E \otimes \Omega_{Y}^{p, q}$, which we denote by $(\cdot, \cdot)_{h, g}$. The space of $(p, q)$-forms with compact support is denoted by $A_{c}^{p, q}(E)$. For any $\eta_{1}, \eta_{2} \in A_{c}^{p, q}(E)$, we put as follows:

$$
\left\langle\eta_{1}, \eta_{2}\right\rangle_{h}=\int\left(\eta_{1}, \eta_{2}\right)_{h, g} \cdot \mathrm{dvol}, \quad\|\eta\|_{h}^{2}=\langle\eta, \eta\rangle_{h} .
$$

The completion of $A_{c}^{p, q}$ with respect to the norm $\|\cdot\|_{h}$ is denoted by $A_{h}^{p, q}$.

We have the operator $\bar{\partial}_{E}: A_{c}^{p, q}(E) \longrightarrow A_{c}^{p, q+1}(E)$, and the formal adjoint $\bar{\partial}_{E}^{*}: A_{c}^{p, q}(E) \longrightarrow A_{c}^{p, q-1}(E)$. We use the notation $\Delta^{\prime \prime}=$ $\bar{\partial}_{E}^{*} \bar{\partial}_{E}+\bar{\partial}_{E} \bar{\partial}_{E}^{*}$. We have the maximal closed extensions $\bar{\partial}_{E}: A_{h}^{p, q}(E) \longrightarrow$ $A_{h}^{p, q+1}(E)$ and $\bar{\partial}_{E}^{*}: A_{h}^{p, q}(E) \longrightarrow A_{h}^{p, q-1}(E)$. We denote the domains of $\bar{\partial}_{E}$ and $\bar{\partial}_{E}^{*}$ by $\operatorname{Dom}\left(\bar{\partial}_{E}\right)$ and $\operatorname{Dom}\left(\bar{\partial}_{E}^{*}\right)$ respectively.

Proposition 4.3 (Proposition 5 of [2]). In $W^{p, q}:=\operatorname{Dom}\left(\bar{\partial}_{E}\right) \cap$ $\operatorname{Dom}\left(\bar{\partial}_{E}^{*}\right)$, the space $A_{c}^{p, q}(E)$ is dense with respect to the the graph norm: $\|\eta\|_{h}^{2}+\left\|\bar{\partial}_{E} \eta\right\|_{h}^{2}+\left\|\bar{\partial}_{E}^{*} \eta\right\|_{h}^{2}$. (See also [11]).

Proposition 4.4 (Theorem 21 of [2]). Assume that there exists a positive number $c>0$ satisfying the following:

Then, for any $\eta \in W^{p, q}$, we have $\left\|\bar{\partial}_{E} \eta\right\|_{h}^{2}+\left\|\bar{\partial}_{E}^{*} \eta\right\|_{h}^{2} \geq c \cdot\|\eta\|_{h}^{2}$. 
For any $C^{\infty}$-element $\eta \in A_{h}^{p, q}(E)$ such that $\bar{\partial}_{E}(\eta)=0$, we have a $C^{\infty}$ solution $\rho \in A_{h}^{p, q-1}(E)$ satisfying the equation $\bar{\partial}_{E}(\rho)=\eta$.

\subsubsection{Kodaira identity}

For the Kahler manifold $Y$, we have the operator $\Lambda: \Omega^{p, q} \longrightarrow \Omega^{p-1, q-1}$ (see 62 page of [26]). For a section $f$ of End $(E) \otimes \Omega_{Y}^{p_{0}, q_{0}}$, we have the natural morphism $A_{c}^{p, q}(E) \longrightarrow A_{c}^{p+p_{0}, q+q_{0}}(E)$, defined by $\eta \longmapsto f \wedge \eta$. We denote the morphism by $e(f)$.

We have the metric connection of $E$ induced by the holomorphic structure $\bar{\partial}_{E}$ and the hermitian metric $h$. We denote the curvature by $R(h)$. We have the Levi-Civita connection of the tangent bundle of $Y$. It induces the connection of $E \otimes \Omega^{0,1}$ :

$$
\nabla: A_{c}^{0,0}\left(E \otimes \Omega^{0,1}\right) \longrightarrow A_{c}^{0,1}\left(E \otimes \Omega^{0,1}\right) \oplus A_{c}^{1,0}\left(E \otimes \Omega^{0,1}\right) .
$$

We denote the $(0,1)$-part of $\nabla$ by $\nabla^{\prime \prime}$ to distinguish with $\bar{\partial}_{E}: A_{c}^{0,1}(E)$ $\longrightarrow A_{c}^{0,2}$. The $(1,0)$-part of $\nabla$ is same as $\partial$ of $E \otimes \Omega^{0,1}$. We denote the curvature of $\nabla$ by $R(\nabla)$.

We denote the Ricci curvature of the Kahler metric $g$ by $\operatorname{Ric}(g)$. We can naturally regard $\operatorname{Ric}(g)$ as a section of $\operatorname{End}(E) \otimes \Omega^{1,1}$, by the natural diagonal inclusion $\mathbf{C} \longrightarrow$ End $(E)$.

Let $f$ be a section of End $(E) \otimes \Omega_{Y}^{1,1}$, and $\eta$ be an element of $A_{c}^{0,1}(E)$. Then we put as follows:

$$
\begin{gathered}
\langle\langle f, \eta\rangle\rangle_{h}:=-\sqrt{-1}(\xi, \eta)_{h}, \\
\xi:=(\Lambda \circ e(f)-e(\Lambda(f)))(\eta)=\Lambda(f \cdot \eta)-\Lambda(f) \cdot \eta .
\end{gathered}
$$

Let $\varphi_{i}$ be an $C^{\infty}$-orthogonal local coframe $\left(\varphi_{i}\right)$ of the tangent bundle of $Y$, that is $g=\sum \varphi_{i} \cdot \bar{\varphi}_{i}$. We also take a $C^{\infty}$-orthogonal local frame $\left(e_{i}\right)$ of $E$. We denote the dual frame by $\left(e_{i}^{\vee}\right)$. We have the local description:

$$
\eta=\sum \eta_{\mu, i} \cdot e_{\mu} \otimes \bar{\varphi}_{i}, \quad f=\sum f_{\mu, \nu, i, \bar{j}} \cdot e_{\mu}^{\vee} \otimes e_{\nu} \otimes\left(\varphi_{i} \wedge \bar{\varphi}_{j}\right)
$$

Then we have the following local description ((9.1) in [11]):

$$
\langle\langle f, \eta\rangle\rangle_{h}:=\sum f_{\mu, \nu, i, \bar{j}} \cdot \eta_{\mu, i} \cdot \bar{\eta}_{\nu, j}
$$

We recall the identity which is called Kodaira identity in [11]. We only need the following special case. 
Proposition 4.5 (Kodaira [27], Cornalba-Griffiths [11]). Let $\eta$ be an element of $A_{c}^{0,1}(E)$. We have the following equality:

$$
\left\|\bar{\partial}_{E}(\eta)\right\|_{h}^{2}+\left\|\bar{\partial}_{E}^{*}(\eta)\right\|_{h}^{2}=\left\|\nabla^{\prime \prime} \eta\right\|^{2}+\int\langle\langle R(h)+\operatorname{Ric}(g), \eta\rangle\rangle_{h} \mathrm{dvol} .
$$

Proof. (See [33] for some formulas of Nakano type.) We have the equality:

$$
\left\|\bar{\partial}_{E}(\eta)\right\|_{h}^{2}+\left\|\bar{\partial}_{E}^{*}(\eta)\right\|_{h}^{2}=\left\langle\Delta^{\prime \prime} \eta, \eta\right\rangle_{h}
$$

On the $(0,1)$-forms, we also have the equality:

$$
\Delta^{\prime \prime}=\partial \partial^{*}+\partial^{*} \partial-\sqrt{-1} \Lambda \circ e(R(h)) .
$$

On $A_{c}^{0,1}(E)$, we have $\partial^{*}=0$ for an obvious reason. Thus we obtain the following:

$$
\left\langle\Delta^{\prime \prime} \eta, \eta\right\rangle_{h}=\langle\partial \eta, \partial \eta\rangle_{h}-\sqrt{-1}\langle\Lambda(R(h) \cdot \eta), \eta\rangle_{h}
$$

We also have the operator on $A_{c}^{0,0}\left(E \otimes \Omega^{0,1}\right)$ :

$$
\Delta_{1}^{\prime \prime}=\nabla^{\prime \prime} \nabla^{\prime \prime *}+\nabla^{\prime \prime *} \nabla^{\prime \prime}=\partial \partial^{*}+\partial^{*} \partial-\sqrt{-1} \Lambda \circ e(R(\nabla)) .
$$

In this case, we have $\Lambda \circ e(R(\nabla))(\eta)=\Lambda(R(\nabla)) \cdot \eta$. Thus we obtain the following:

$$
\begin{aligned}
\left\langle\Delta^{\prime \prime} \eta, \eta\right\rangle_{h}= & \left\|\nabla^{\prime \prime} \eta\right\|_{h}^{2}-\sqrt{-1}\langle\Lambda(R(h) \cdot \eta), \eta\rangle_{h} \\
& +\sqrt{-1}\langle\Lambda(R(\nabla)) \cdot \eta, \eta\rangle_{h} .
\end{aligned}
$$

We have $R(\nabla)=R(h)+R\left(\Omega^{0,1}\right)$. It can be checked that $\sqrt{-1}\left(\Lambda\left(R\left(\Omega^{0,1}\right)\right.\right.$. $\eta), \eta)_{h}$ is same as $\langle\langle\operatorname{Ric}(g), \eta\rangle\rangle$, by a direct calculation and the coincidence of the Ricci curvature and the mean curvature of the Kahler metric (See (7.23) in page 28 of [26].) Thus we obtain the following:

$$
\begin{aligned}
\left\langle\Delta^{\prime \prime} \eta, \eta\right\rangle_{h}=\left\|\nabla^{\prime \prime} \eta\right\|_{h}^{2}+\int[- & \sqrt{-1}(\Lambda(R(h) \cdot \eta)-\Lambda(R(h)) \cdot \eta, \eta)_{h} \\
& \left.+\langle\langle\operatorname{Ric}(g), \eta\rangle\rangle_{h}\right] \mathrm{dvol} .
\end{aligned}
$$

Thus we are done.

q.e.d. 
Corollary 4.11. Let $\eta$ be an element of $\operatorname{Dom}\left(\bar{\partial}_{E}\right) \cap \operatorname{Dom}\left(\bar{\partial}_{E}^{*}\right)$ in $A_{h}^{0,1}(E)$. Then we have the following inequality:

$$
\left\|\bar{\partial}_{E}(\eta)\right\|_{h}^{2}+\left\|\bar{\partial}_{E}^{*}(\eta)\right\|_{h}^{2} \geq \int\langle\langle R(h)+\operatorname{Ric}(g), \eta\rangle\rangle_{h} \mathrm{dvol} .
$$

Proof. We only have to note the density of $A_{c}^{0,1}(E)$ in $\operatorname{Dom}\left(\bar{\partial}_{E}\right) \cap$ $\operatorname{Dom}\left(\bar{\partial}_{E}^{*}\right)$ (Proposition 4.3).

\subsubsection{The modification of acceptable metrics}

Consider the Poincare metric $g_{\mathbf{p}}$ on $Y=\Delta^{* l} \times \Delta^{n-l}$, and the acceptable hermitian holomorphic vector bundle $\left(E, \bar{\partial}_{E}, h\right)$ (Definition 4.3).

Let $\boldsymbol{a}=\left(a_{1}, \ldots, a_{l}\right)$ be a tuple of real numbers. Let $N$ be a real number. Then we put as follows:

$$
\begin{aligned}
\tau(\boldsymbol{a}, N):= & \sum_{i=1}^{l} a_{i} \log \left|z_{i}\right|^{2} \\
& +N \cdot\left(\sum_{i=1}^{l} \log \left(-\log \left|z_{i}\right|^{2}\right)+\sum_{i=l+1}^{n} \log \left(1-\left|z_{i}\right|^{2}\right)\right) .
\end{aligned}
$$

Recall the following formulas:

$$
\begin{gathered}
\bar{\partial} \partial \log |z|^{2}= \\
0, \quad \bar{\partial} \partial \log \left(-\log |z|^{2}\right)=\frac{d z \cdot d \bar{z}}{\left(-\log |z|^{2}\right)^{2} \cdot|z|^{2}}, \\
\bar{\partial} \partial \log \left(1-|z|^{2}\right)=\frac{d z \cdot d \bar{z}}{\left(1-\left|z_{i}\right|^{2}\right)^{2}} .
\end{gathered}
$$

For a metric $h$, we put as follows:

$$
\begin{aligned}
h_{\boldsymbol{a}, N}: & =h \cdot \exp (-\tau(\boldsymbol{a}, N)) \\
& =h \times \prod_{i=1}^{l}\left|z_{i}\right|^{-2 a_{i}}\left(-\log \left|z_{i}\right|\right)^{-N} \times \prod_{i=l+1}^{n}\left(1-\left|z_{i}\right|^{2}\right)^{-N} .
\end{aligned}
$$

We use the notation $|\cdot|_{\boldsymbol{a}, N},\|\cdot\|_{\boldsymbol{a}, N},(\cdot, \cdot)_{\boldsymbol{a}, N}$ and $\langle\langle\cdot, \cdot\rangle\rangle_{\boldsymbol{a}, N}$ instead of $|\cdot|_{h_{a, N}},\|\cdot\|_{h_{a, N}},(\cdot, \cdot)_{h_{a, N}}$ and $\langle\langle\cdot, \cdot\rangle\rangle_{h_{a, N}}$ for simplicity. We also use the notation $A_{\boldsymbol{a}, N}^{p, q}(E)$ instead of $A_{h_{a, N}}^{p, q}(E)$.

The (1,0)-part of the metric connection for the $h_{\boldsymbol{a}, N}$ is denoted by $\partial_{\boldsymbol{a}, N}$. The curvature is denoted by $R\left(h_{\boldsymbol{a}, N}\right)$. We have the following 
formula for $R\left(h_{\boldsymbol{a}, N}\right)$ :

$$
\begin{aligned}
R\left(h_{\boldsymbol{a}, N}\right) & =R(h)-\bar{\partial} \partial \tau(\boldsymbol{a}, N) \\
& =R(h)-N \cdot\left(\sum_{i=1}^{l} \frac{d z_{i} \cdot d \bar{z}_{i}}{\left(-\log \left|z_{i}\right|^{2}\right)^{2}\left|z_{i}\right|^{2}}+\sum_{i=l+1}^{n} \frac{d z_{i} \cdot d \bar{z}_{i}}{\left(1-\left|z_{i}\right|^{2}\right)^{2}}\right)
\end{aligned}
$$

In particular, we have the equality $\sqrt{-1} \Lambda\left(R\left(h_{\boldsymbol{a}, N}\right)\right)=\sqrt{-1} \Lambda(R(h))-$ $n \cdot N$.

We will use the following later.

Lemma 4.12. When $N$ is sufficiently smaller than 0 , then the following inequality holds for any $(0,1)$-form $\eta$ :

$$
\left\langle\left\langle R\left(h_{\boldsymbol{a}, N}\right)+\operatorname{Ric}(g), \eta\right\rangle\right\rangle_{\boldsymbol{a}, N} \geq|\eta|_{\boldsymbol{a}, N}^{2}
$$

Proof. It immediately follows from the equality (36) and the local description (35).

q.e.d.

Remark 4.4. The real number $N$ has no effect to the increasing order of holomorphic sections at the divisors. Namely, if we have a holomorphic section $s$, the increasing order with respect to $h_{\boldsymbol{a}, N}$ is independent of $N$. On the contrary, $\boldsymbol{a}$ has an effect.

\subsubsection{When $N$ is sufficiently larger than 0 .}

Consider the case $N$ is sufficiently larger than 0 . The function $\tau(\boldsymbol{a}, N)$ diverges at the boundary of $Y$. Thus we consider $Y(C):=\Delta^{*}(C)^{l} \times$ $\Delta(C)^{n-l}$ for a number $0<C<1$. The projection of $\Delta^{*}(C)^{l} \times \Delta(C)^{n-l}$ $\longrightarrow \Delta^{*}(C)^{l-1} \times \Delta(C)^{n-l}$, forgetting $j$-th component $(1 \leq j \leq l)$, is denoted by $\pi_{j}$. For any element $p \in \Delta^{*}(C)^{l-1} \times \Delta(C)^{n-l}$, we obtain the curve $\pi_{j}^{-1}(p) \subset Y(C)$, which is isomorphic to the punctured disc.

Let $s$ be a holomorphic section of $E$ of $Y(C)$. Consider the restriction of $F$ to the curve $\pi_{j}^{-1}(p)$. We denote the restriction by $F_{\mid \pi_{j}^{-1}(p)}$. On 
$\pi_{j}^{-1}(p)$, we have the following formula:

$$
\begin{aligned}
\Delta_{\mathbf{p}}^{\prime \prime} \mid & F_{\mid \pi_{j}^{-1}(p)} \mid \boldsymbol{a}, N \\
= & -\left|\partial_{\boldsymbol{a}, N} F_{\mid \pi_{j}^{-1}(p)}\right|_{\boldsymbol{a}, N}^{2}+\left(F_{\mid \pi_{j}^{-1}(p)}, \sqrt{-1} \Lambda R\left(h_{\boldsymbol{a}, N}\right) \cdot F_{\mid \pi_{j}^{-1}(p)}\right)_{\boldsymbol{a}, N} \\
= & -\left|\partial_{\boldsymbol{a}, N} F_{\mid \pi_{j}^{-1}(p)}\right|_{\boldsymbol{a}, N}^{2}+\left(F_{\mid \pi_{j}^{-1}(p)}, \sqrt{-1} \Lambda R(h) \cdot F_{\mid \pi_{j}^{-1}(p)}\right)_{\boldsymbol{a}, N} \\
& -n \cdot N \cdot\left|F_{\pi_{j}^{-1}(p)}\right|_{\boldsymbol{a}, N}^{2} .
\end{aligned}
$$

Here $\Delta_{\mathbf{p}}^{\prime \prime}$ denotes the Laplacian for the punctured disc $\pi_{j}^{-1}(p)$ with the Poincare metric. Then we obtain the following inequality:

$$
\begin{aligned}
& \Delta_{\mathbf{p}}^{\prime \prime} \log \left|F_{\pi_{j}^{-1}(p)}\right|_{\boldsymbol{a}, N}^{2} \\
& \leq \frac{\Delta_{\mathbf{p}}^{\prime \prime}\left|F_{\mid \pi_{j}^{-1}(p)}\right|_{\boldsymbol{a}, N}^{2}}{\left|F_{\mid \pi_{j}^{-1}(p)}\right|_{\boldsymbol{a}, N}^{2}}+\frac{\left|\left(\partial_{\boldsymbol{a}, N} F_{\mid \pi_{j}^{-1}(p)}, F_{\mid \pi_{j}^{-1}(p)}\right) \boldsymbol{a}_{\boldsymbol{a}, N}\right|^{2}}{\left|F_{\mid \pi_{j}^{-1}(p)}\right|_{\boldsymbol{a}, N}^{4}} \\
& \leq-n \cdot N+|R|_{h, g_{\mathbf{p}}} .
\end{aligned}
$$

If $\left(E, \bar{\partial}_{E}, h\right)$ is acceptable, then $|R|_{h, g_{\mathbf{p}}}$ is bounded by definition, and thus there exists a positive integer $N_{0}$ such that $-n \cdot N+|R|_{h, g_{\mathbf{p}}}<0$ for any $N>N_{0}$. Then we obtain the following inequality for any holomorphic section $F$ and for any $N>N_{0}$ :

$$
\Delta_{\mathbf{p}}^{\prime \prime} \log \left|F_{\mid \pi_{j}^{-1}(p)}\right|_{\boldsymbol{a}, N}^{2}<0 .
$$

We can take such $N$ independently of $p, j$ and $F$. We can replace $\Delta_{\mathbf{p}}^{\prime \prime}$ with the standard Laplacian $-\left(\partial_{s}^{2}+\partial_{t}^{2}\right)$ for the real coordinate $z_{j}=s+\sqrt{-1} t$.

As an example, we obtain the following corollary. It says, we obtain the increasing order of a holomorphic section over $\Delta^{* l} \times \Delta^{n-l}$ from the increasing order of the restriction to the curves.

Corollary 4.12. Let $F$ be a holomorphic section of $\mathcal{E}^{\lambda}$. Let $a_{j}$ and $k_{j}$ be real numbers $(j=1, \ldots, l)$.

For any point $p \in \Delta^{*}(C)^{l-1} \times \Delta(C)^{n-l}$ and any $1 \leq j \leq l$, we assume that we are given numbers $C_{1}(p, j), C_{2}(p, j), a(p, j)$ and $k(p, j)$ satisfying the following:

1. $C_{1}(p, j)$ and $C_{2}(p, j)$ are positive numbers.

2. $a(p, j)$ and $k(p, j)$ are real numbers satisfying $a(p, j) \leq a_{j}$ and $k(p, j) \leq k_{j}$. 
3. The following inequality holds on $\pi_{j}^{-1}(p)$ :

$$
0<C_{1}(p, j) \leq\left|F_{\mid \pi_{j}^{-1}(p)}\right| h \cdot\left|z_{j}\right|^{-a(p, j)} \cdot\left(-\log \left|z_{j}\right|\right)^{-k(p, j)} \leq C_{2}(p, j) .
$$

4. $C_{1}(p, j), C_{2}(p, j), a(p, j)$ and $k(p, j)$ may depend on $p$ and $j$.

Then there exists a positive constant $C_{3}$ and a large number $M$, satisfying the following:

- The inequality $|s|_{h} \leq C_{3} \cdot \prod_{j=1}^{l}\left|z_{j}\right|^{a_{j}}\left(-\log \left|z_{j}\right|\right)^{M}$ holds over $Y(C)$.

- $C_{3}$ depends only on the values of $|s|_{h}$ at $\left\{\left(z_{1}, \ldots, z_{n}\right)|| z_{j} \mid=C\right.$, $j=1, \ldots, l\}$.

Proof. We put $\boldsymbol{a}=(0, \ldots, 0)$. Pick $p \in \Delta^{*}(C)^{l-1} \times \Delta(C)^{n-l}$ and $1 \leq j \leq l$. We note that the following holds: By our assumption, we have the following for any number $N$ :

$$
\lim _{\left|z_{j}\right| \rightarrow 0} \frac{\log \left|F_{\mid \pi_{j}^{-1}(p)}\right| a, N-a \log \left|z_{j}\right|}{\log \left|z_{j}\right|}=0 .
$$

If $N$ is so large that $-n \cdot N+|R|_{h, g_{\mathbf{p}}}<0$, then we obtain the following inequality on $\pi_{j}^{-1}(a)$ :

$$
\Delta\left(\log \left|F_{\mid \pi_{j}^{-1}(p)}\right| a, N-a \log \left|z_{j}\right|\right) \leq 0 .
$$

Here $\Delta$ denotes the standard Laplacian $-\left(\partial_{s}^{2}+\partial_{t}^{2}\right)$ on $\pi_{j}^{-1}(p)$. Then the values of $\log \left|F_{\mid \pi_{j}^{-1}(p)}\right|-a \log \left|z_{j}\right|$ on $\pi_{j}^{-1}(p)$ are dominated by the values at $\left\{\left|z_{j}\right|=C\right\} \cap \pi_{j}^{-1}(p)$. (See [35], in particular, Lemma 2.2 and the proof of Corollary 4.2). Thus we obtain the following inequality:

$$
\left|F_{\mid \pi_{j}^{-1}(p)}\right| \boldsymbol{a}, N \leq C(p, j) \cdot\left|z_{j}\right|^{a} .
$$

Here $C(p, j)$ denotes a constant depending only on the values $F$ at $\left\{\left|z_{j}\right|=C\right\} \cap \pi_{j}^{-1}(p)$ :

$$
C(p, j)=\max \left\{\left|F_{\mid \pi_{j}^{-1}(p)}\left(z_{j}\right)\right|_{a, N}\left|z_{j} \in \mathbf{C},\right| z_{j} \mid=C\right\} .
$$

From the inequality (39), we obtain the following on $\pi_{j}^{-1}(p)$ :

$$
\left|F_{\mid \pi_{j}^{-1}(p)}\right|_{h} \leq C(p, j) \cdot\left|z_{j}\right|^{a_{j}} \cdot\left(-\log \left|z_{j}\right|\right)^{N} .
$$

Then we obtain the result by using an induction on $l$.

q.e.d.

We also have the following: 
Lemma 4.13. Let $(E, h)$ be a hermitian holomorphic bundle over $\Delta^{* l} \times \Delta^{n-l}$. Let $F$ be a holomorphic section of $E$ over $\Delta^{* l} \times \Delta^{n-l}$. Assume that $\|F\|_{\boldsymbol{a}, N}<\infty$. If $N$ is sufficiently large, then $\left\|F_{\mid \pi_{j}^{-1}(p)}\right\|_{\boldsymbol{a}, N}<$ $\infty$ for any $j$ and $p \in \Delta^{* l-1} \times \Delta^{n-l}$.

Proof. We only have to use the subharmonicity of the function $\left|F_{\mid \pi_{j}^{-1}(a)}\right|_{\boldsymbol{a}, N}$.

q.e.d.

\subsection{Some reductions toward the prolongation of the de- formed holomorphic bundles}

\subsubsection{The prolongation of $\mathcal{E}^{\lambda}$ when $\lambda \neq 0$}

Let $\left(E, \bar{\partial}_{E}, \theta, h\right)$ be a tame nilpotent harmonic bundle with trivial parabolic structure over $\Delta^{* l} \times \Delta^{n-l}$. Consider the prolongation of $\mathcal{E}^{\lambda}$ over $\Delta^{* l} \times \Delta^{n-l}$ by increasing order $(0, \ldots, 0)$.

Since the eigenvalues of the monodromies are 1 , we can take a normalizing frame $\boldsymbol{w}$ of $\mathcal{E}^{\lambda}$ over $\Delta^{* l} \times \Delta^{n-l}$, namely, the connection form $A$ of $\mathbb{D}^{\lambda, f}$ with respect to $\boldsymbol{w}=\left(w_{1}, \ldots, w_{r}\right)$ satisfies the following (see Subsubsection 5.1.1):

- $A$ is of the form $A=\sum_{j=1}^{l} A_{j} \cdot d z_{j} / z_{j}$ for some constant matrices $A_{j} \in M(r)$.

- All of the eigenvalues of $A_{j}$ are 0 .

Note that the restriction $\boldsymbol{w}_{\mid \pi_{j}^{-1}(p)}$ of $\boldsymbol{w}$ to the curves $\pi_{j}^{-1}(p)$ gives a normalizing frame of $\left(\mathcal{E}_{\mid \pi_{j}^{-1}(p)}^{\lambda}, \mathbb{D}_{\mid \pi_{j}^{-1}(p)}^{\lambda}\right)$. Thus $\boldsymbol{w}_{\mid \pi_{j}^{-1}(p)}$ is a frame of ${ }^{\diamond} \mathcal{E}_{\mid \pi_{j}^{-1}(p)}^{\lambda}$, due to Lemma 4.10. In particular, the restrictions $w_{i \mid \pi_{j}^{-1}(p)}$ satisfies the conditions of Corollary 4.12. Thus we can conclude that the increasing order of $w_{i}$ is less than $(0, \ldots, 0)$, i.e., $w_{i}$ is a holomorphic section of ${ }^{\diamond} \mathcal{E}^{\lambda}$.

On the other hand, consider the following section of $\operatorname{det}\left(\mathcal{E}^{\lambda}\right)$ over $\Delta^{* l} \times \Delta^{n-l}$ :

$$
\Omega(\boldsymbol{w})=w_{1} \wedge \cdots \wedge w_{r} .
$$

By our choice, we know that $\mathbb{D}^{\lambda}(\Omega(\boldsymbol{w}))=0$. Thus it naturally gives a holomorphic frame of ${ }^{\diamond} \operatorname{det}\left(\mathcal{E}^{\lambda}\right)$ over $\Delta^{n}$.

Lemma 4.14. Let $s=\left(s_{1}, \ldots, s_{r}\right)$ be a tuple of holomorphic sections of the sheaf ${ }^{\diamond} \mathcal{E}^{\lambda}$. 
1. The section $\Omega(s)=s_{1} \wedge \cdots \wedge s_{r}$ over $\Delta^{* l} \times \Delta^{n-l}$ naturally induces the section of ${ }^{\diamond} \operatorname{det}\left(\mathcal{E}^{\lambda}\right)$. We denote the section by the same notation.

2. Assume that $\Omega(s)(P) \neq 0$ in ${ }^{\diamond} \operatorname{det}\left(\mathcal{E}^{\lambda}\right)_{\mid P}$. Then ${ }^{\diamond} \mathcal{E}^{\lambda}$ is locally free around $P$, and $s$ gives a holomorphic frame around $P$.

Proof. The first claim is clear, for we only have to see the increasing order of $\Omega(s)$.

Assume that $\Omega(s)(P) \neq 0$. Then we have an open subset $U$ of $\Delta^{n}$ such that $\Omega(s)$ gives a frame of ${ }^{\diamond} \operatorname{det}\left(\mathcal{E}^{\lambda}\right)$ over $U$. On the open set $U^{\prime}=U \cap\left(\Delta^{* l} \times \Delta^{n-l}\right)$, the tuple $s$ gives a holomorphic frame of $\mathcal{E}^{\lambda}$. Let $f$ be an element of $\Gamma\left(U, \diamond \mathcal{E}^{\lambda}\right)$. Since we have already known that $s$ gives a frame on $U^{\prime}$, we have a holomorphic functions $f_{i}$ defined over $U^{\prime}$ satisfying the following over $U^{\prime}$ :

$$
f=\sum f_{i} \cdot s_{i}
$$

Consider $f_{1}$. We know that $f \wedge s_{2} \wedge \ldots s_{r}=f_{1} \cdot \Omega(s)$ gives a section of ${ }^{\diamond} \operatorname{det}\left(\mathcal{E}^{\lambda}\right)$ on $U$. We also know that $\Omega(s)$ gives a frame of ${ }^{\diamond} \operatorname{det}\left(\mathcal{E}^{\lambda}\right)$ on $U$. Thus we can conclude that $f_{1}$ is in fact a holomorphic function over $U$. Similarly we can show that the other $f_{i}$ are also holomorphic over $U$. q.e.d.

As a corollary, we obtain the following:

Corollary 4.13. When $\lambda \neq 0$, then ${ }^{\diamond} \mathcal{E}^{\lambda}$ is locally free coherent sheaf, and the normalizing frame gives a frame.

Corollary 4.14. The $\lambda$-connection $\mathbb{D}^{\lambda}$ and the flat connection $\mathbb{D}^{\lambda, f}$ is of log type on $\diamond \mathcal{E}^{\lambda}$.

Remark 4.5. The argument of Lemma 4.14, which we learn at [11], will be used without mention.

Let $\boldsymbol{v}$ be a holomorphic frame of ${ }^{\diamond} \mathcal{E}^{1}$ over $\Delta^{n}$. Consider the $\mathcal{H}(r)$ valued function $H(h, \boldsymbol{v})$.

Lemma 4.15. There exists a large number $M$ and positive numbers $C_{i}(i=1,2)$ satisfying the following:

$$
C_{1} \cdot \prod_{i=1}^{l}\left(-\log \left|z_{i}\right|\right)^{-M} \leq H(h, \boldsymbol{v}) \leq C_{2} \cdot \prod_{i=1}^{l}\left(-\log \left|z_{i}\right|\right)^{M} .
$$


Proof. We only have to consider the case that $\boldsymbol{v}$ is a normalizing frame. We have already known the following estimate for some positive number $C_{3}$ and $M_{1}$ :

$$
\left|v_{i}\right| \leq C_{3} \cdot \prod_{i=1}^{l}\left(-\log \left|z_{i}\right|\right)^{M_{1}} .
$$

Thus we obtain the right inequality in (40). To see the left inequality, we use the dual. Namely let $\boldsymbol{v}^{\vee}$ denote the dual frame of $\boldsymbol{v}$. Then $\boldsymbol{v}^{\vee}$ is also a normalizing frame of $\mathcal{E}^{1 \vee}$. Hence it satisfies the inequality similar to (41). Hence we obtain the right inequality for $H\left(h^{\vee}, \boldsymbol{v}^{\vee}\right)$. Since we have $H\left(h^{\vee}, \boldsymbol{v}^{\vee}\right) \cdot H(h, \boldsymbol{v})=1$, we obtain the result.

q.e.d.

Remark 4.6. The argument of the proof of Lemma 4.15 works for any $\lambda \neq 0$. If $\lambda=0$, we do not have a normalizing frame, in general. However, the argument works once we know that the dual frame $\boldsymbol{v}^{\vee}$ of $\mathcal{E}^{0 \vee}$ gives a frame of $\diamond \mathcal{E}^{0 \vee}$.

\subsubsection{The prolongation of $\mathcal{E}^{\sharp}$}

We put $X=\Delta^{n}=\left\{\left(z_{1}, \ldots, z_{n}\right)|| z_{i} \mid<1\right\}$ and $D=\bigcup_{i=1}^{l} D_{i}$, where $D_{i}=\left\{z_{i}=0\right\}$. Pick $\lambda_{0} \in \mathbf{C}_{\lambda}$. Let $\mathcal{O}\left(-\left\{\lambda_{0}\right\}\right)$ denote the sheaf of the holomorphic functions which vanish at the divisor $\mathcal{X}^{\lambda_{0}}$. The sheaf $\mathcal{O}\left(-\left\{\lambda_{0}\right\}\right)$ is a line bundle over $\mathcal{X}$. We have the natural inclusion $\mathcal{O}\left(\left(-\left\{\lambda_{0}\right\}\right)\right) \subset \mathcal{O}$. The restriction of $\mathcal{O}\left(-\left\{\lambda_{0}\right\}\right)$ to an open subset of $\mathcal{X}$ is denoted by the same notation.

We put $\mathcal{E}\left(-\left\{\lambda_{0}\right\}\right):=\mathcal{E} \otimes \mathcal{O}\left(-\left\{\lambda_{0}\right\}\right)$ We have the natural inclusion: $\mathcal{E}\left(-\left\{\lambda_{0}\right\}\right) \subset \mathcal{E}$.

We put $\Delta_{0}:=\left\{\lambda \in \mathbf{C}_{\lambda}|| \lambda-\lambda_{0} \mid<1\right\}$. We can naturally identify $\Delta_{0}$ with $\Delta$. We denote the projection of $\Delta_{0} \times(X-D)$ onto $X-D$ by $p_{\lambda}$. We denote the restriction of $\mathcal{E}$ to $\Delta_{0} \times X$ by the same notation $\mathcal{E}$, for simplicity.

Lemma 4.16. Let $f$ be a holomorphic section of ${ }^{\diamond}\left(\mathcal{E}_{\mid \mathcal{X}^{\lambda_{0}}}\right)$ over $\mathcal{X}^{\lambda_{0}}$. Then there exists a holomorphic section $\widetilde{f}$ of ${ }^{\diamond} \mathcal{E}$ over $\Delta_{0} \times X$, satisfying $\widetilde{f}_{\mid \mathcal{X}^{\lambda_{0}}}=f$.

Proof. We use a standard argument. Let $\chi$ be a $C^{\infty}$-function defined over $\Delta_{0}$, satisfying the following:

$$
\chi(\lambda)= \begin{cases}1 & \left(\left|\lambda-\lambda_{0}\right|<1 / 3\right) \\ 0 & \left(\left|\lambda-\lambda_{0}\right|>2 / 3\right) .\end{cases}
$$


Let $d^{\prime \prime}$ denote the holomorphic structure of $\mathcal{E}$. For simplicity, we denote $p_{\lambda}^{-1}\left(f_{\mid \mathcal{X}^{\lambda_{0}}-\mathcal{D}^{\lambda_{0}}}\right)$ by $p_{\lambda}^{-1}(f)$. Then the $(0,1)$-form $\eta$ defined over $\Delta_{0} \times$ $(X-D)$ is defined as follows:

$$
\eta:=d^{\prime \prime}\left(\chi \cdot p_{\lambda}^{-1}(f)\right)=\bar{\partial} \chi \cdot p_{\lambda}^{-1}(f)+\chi \cdot\left(\lambda-\lambda_{0}\right) \cdot p_{\lambda}^{-1}\left(\theta^{\dagger} f\right) .
$$

Take a sufficiently negative number $N$ as in Lemma 4.12. Let $\epsilon$ be any sufficiently small positive number. Let $\boldsymbol{\delta}$ denote the tuple $\overbrace{(1, \ldots, 1)}^{l}$. By our construction, we have $d^{\prime \prime} \eta=0$. Due to the estimate of $\theta^{\dagger}$ (Proposition 4.1) and our assumption on the increasing order of $f$, we know that $\eta$ is an element of $A_{-\epsilon \cdot \delta, N}^{0,1}(\mathcal{E})$. Moreover, we have $\bar{\partial} \chi=0$ on $\left\{\left|\lambda-\lambda_{0}\right|<1 / 3\right\}$. Hence $\eta$ is, in fact, an element of $A_{-\epsilon \cdot \boldsymbol{\delta}, N}^{0,1}\left(\mathcal{E}\left(\left\{-\lambda_{0}\right\}\right)\right)$. Then we can pick an element $\rho$ of $A_{-\epsilon \cdot \boldsymbol{\delta}, N}^{0,0}\left(\mathcal{E} \otimes \mathcal{O}\left(-\left\{\lambda_{0}\right\}\right)\right)$ over $\Delta_{0} \times(X-$ $D$ ), satisfying $d^{\prime \prime} \rho=\eta$ (Proposition 4.3, Proposition 4.4, Corollary 4.11, Lemma 4.12).

We put $\widetilde{f}:=\chi \cdot p_{\lambda}^{-1}(f)-\rho$, where we regard $\rho$ as an element of $A_{-\epsilon \cdot \delta, N}^{0,0}(\mathcal{E})$ by the inclusion $\mathcal{E}\left(-\left\{\lambda_{0}\right\}\right) \longrightarrow \mathcal{E}$. By our construction, it is easy to check the following:

1. $\tilde{f}$ is holomorphic section of $\mathcal{E}$ over $\Delta_{0} \times(X-D)$.

2. The restriction of $\tilde{f}$ to $\left\{\lambda_{0}\right\} \times(X-D)$ is same as $f$.

3. $\tilde{f}$ is an element of $A_{-\epsilon \cdot \boldsymbol{\delta}, N}^{0,0}(\mathcal{E})$.

We show that the increasing order of $f$ with respect to $\Delta_{0} \times D$ is less than $(0, \ldots, 0)$. Let $\pi_{j}$ denote the projection $X-D \longrightarrow \Delta^{* l-1} \times \Delta^{n-l}$, forgetting $j$-th component for $1 \leq j \leq l$. Consider the restriction of $\tilde{f}$ to $\{\lambda\} \times \pi_{j}^{-1}(p)$ for any $p \in \Delta^{* l-1} \times \Delta^{n-l}$ and for any $1 \leq j \leq l$. We know that the parabolic structure of $\diamond^{\diamond} \mathcal{E}_{\mid \pi_{j}^{-1}(p)}^{\lambda}$ is trivial, by our assumption. Due to Lemma 4.13 and the norm estimate in one dimensional case, the increasing order of $\widetilde{f}_{\mid\{\lambda\} \times \pi_{j}^{-1}(p)}$ is less than $-\epsilon$. Since $\epsilon$ is sufficiently small, we obtain that the increasing order of the restriction of $\tilde{f}$ to $\{\lambda\} \times \pi_{j}^{-1}(a)$ is, in fact, less than 0 .

Then we can apply Corollary 4.12. (We put $a_{j}=0$ in applying.) Then we obtain that the increasing order of $\widetilde{f}$ with respect to $\Delta_{0} \times D$ is less than $(0, \ldots, 0)$. Namely $\widetilde{f}$ is a section of $\diamond \mathcal{E}^{0}$ over $\Delta_{0} \times X$. q.e.d. 
Proposition 4.6. Let $\left(E, \bar{\partial}_{E}, \theta, h\right)$ be a tame nilpotent harmonic bundle with trivial parabolic structure over $X-D$.

1. The prolongment of the deformed holomorphic bundle ${ }^{\sharp} \sharp$ over $\mathcal{X}^{\sharp}$ is locally free. Here $\mathcal{X}^{\sharp}=\mathbf{C}_{\lambda}^{*} \times X$ and $\mathcal{E}^{\sharp}=\mathcal{E}_{\mid \mathcal{X}^{\sharp}-\mathcal{D}^{\sharp}}$.

2. If ${ }^{\diamond} \mathcal{E}^{0}$ over $X$ is locally free, then ${ }^{\diamond} \mathcal{E}$ over $\mathcal{X}$ is locally free.

Proof. We assume that $\lambda_{0} \neq 0$ or that we know that $\mathcal{E}^{0}$ is locally free. Take a holomorphic frame $\boldsymbol{v}=\left(v_{1}, \ldots, v_{n}\right)$ of ${ }^{\diamond} \mathcal{E}^{\lambda_{0}}$ over $X$. Due to Lemma 4.16, we can take holomorphic sections $\widetilde{v}_{i}$ of $\mathcal{E}$ over $\Delta_{0} \times X$ such that $\widetilde{v}_{i \mid \mathcal{X}^{\lambda_{0}}}=v_{i}$.

Thus we obtain the tuple of holomorphic sections $\widetilde{\boldsymbol{v}}=\left(\widetilde{v}_{i}\right)$ of $\diamond \mathcal{E}$ around $\left\{\lambda_{0}\right\} \times\{O\}$. Here $O$ denotes the origin $(0, \ldots, 0)$ of $X=\Delta^{n}$. By our choice, $\Omega(\widetilde{\boldsymbol{v}})\left(\lambda_{0}, O\right)=\Omega(\boldsymbol{v})\left(\lambda_{0}, O\right)$ is not $0 \operatorname{in}{ }^{\diamond} \operatorname{det}(\mathcal{E})_{\mid\left(\lambda_{0}, O\right)}$. By the same argument as the proof of Lemma 4.14 , we obtain that ${ }^{\diamond} \mathcal{E}$ is locally free on a neighborhood $U$ of $\left(\lambda_{0}, O\right)$, and that $\widetilde{\boldsymbol{v}}$ gives a holomorphic frame on $U$. Thus the proof of Proposition 4.6 is completed. q.e.d.

\subsubsection{One dimensional case, revisited}

Corollary 4.15. Let $\left(E, \bar{\partial}_{E}, \theta, h\right)$ be a tame nilpotent harmonic bundle with trivial parabolic structure over the punctured disc $\Delta^{*}$. Then the prolongment ${ }^{\diamond} \mathcal{E}$ of the deformed holomorphic bundle is locally free. The $\lambda$-connection $\mathbb{D}^{\lambda}$ is of log type.

Proof. We have already known that the prolongment of $\mathcal{E}^{0}$ is coherent locally free. Thus ${ }^{\diamond} \mathcal{E}$ is locally free due to Proposition 4.6.

Let $f$ be a holomorphic section of ${ }^{\diamond} \mathcal{E}$. Consider $\mathbb{D}(f)$. It is holomorphic over $\mathcal{X}-\mathcal{D}$. Since a normalizing frame gives a frame of ${ }^{\diamond} \mathcal{E}^{\sharp}$ over $\mathcal{X}^{\sharp}, \mathbb{D}(f)$ is holomorphic as a section of ${ }^{\diamond} \mathcal{E}^{\sharp} \otimes p_{\lambda}^{*} \Omega_{X}^{1,0}$ over $\mathcal{X}^{\sharp}$. Then $\mathbb{D}(f)$ is a holomorphic section of locally free sheaf ${ }^{\diamond} \mathcal{E} \otimes p_{\lambda}^{*} \Omega_{X}(\log D)$, outside the codimension two subset $\mathcal{D}^{0}$. Thus $\mathbb{D}(f)$ is a holomorphic section of $\diamond \mathcal{E} \otimes p_{\lambda}^{*} \Omega_{X}(\log D)$, over $\mathcal{X}$, namely $\mathbb{D}$ is of $\log$ type. q.e.d.

We have already known that the conjugacy classes of $\operatorname{Res}\left(\mathbb{D}^{\lambda}\right)$ is independent of $\lambda$. Thus the weight filtrations of $\operatorname{Res}\left(\mathbb{D}^{\lambda}\right)$ form the filtration of ${ }^{\diamond} \mathcal{E}_{\mid \mathbf{C}_{\lambda} \times O}$. We denote it by $W$. The associated graded vector bundle over $\mathbf{C}_{\lambda} \times\{O\}$ is denoted by $\mathcal{G} r=\bigoplus_{h} \mathcal{G} r_{h}$. We denote the primitive part of $\mathcal{G} r_{h}$ by $P_{|h|+2 a} \mathcal{G} r_{h}$.

Lemma 4.17. There exists a holomorphic frame $\boldsymbol{v}$ of ${ }^{\diamond} \mathcal{E}$ over $\mathbf{C}_{\lambda} \times$ $\Delta$, satisfying the following: 
- We have the $M(r)$-valued holomorphic function $J(\lambda)$ over $\mathbf{C}_{\lambda} \times$ $\{O\}$, such that $\operatorname{Res}\left(\mathbb{D}^{\lambda}\right) \cdot \boldsymbol{v}_{\mid \mathbf{C}_{\lambda} \times O}=\boldsymbol{v}_{\mid \mathbf{C}_{\lambda} \times O} \cdot J(\lambda)$. Then $J(\lambda)$ is, in fact, a constant matrix $J$.

Proof. For any $h \geq 0$, we have the surjective morphism of vector bundles:

$$
\pi_{h}: \operatorname{Ker}\left(\operatorname{Res}\left(\mathbb{D}^{\lambda}\right)^{h+1}\right) \longrightarrow P_{h} \mathcal{G} r_{h}
$$

We take a splitting $\phi_{h}$ of $\pi_{h}$. We denote the image of $\phi_{h}$ by $P_{h} \mathcal{G} r_{h}^{\prime}$, which is a subbundle of ${ }^{\diamond} \mathcal{E}_{\mathbf{C}_{\lambda} \times O}$. For any $0 \leq a \leq h$, we put $P_{h} \mathcal{G} r_{h-2 a}^{\prime}:=$ $\operatorname{Res}\left(\mathbb{D}^{\lambda}\right)^{a}\left(P_{h} \mathcal{G} r_{h}^{\prime}\right)$. Note that $\operatorname{Res}\left(\mathbb{D}^{\lambda}\right)^{h+1}\left(P_{h} \mathcal{G} r_{h}^{\prime}\right)=0$ due to our choice of $P_{h} \mathcal{G} r_{h}^{\prime}$. Then we obtain a decomposition:

$$
{ }^{\diamond} \mathcal{E}_{\mid \mathbf{C}_{\lambda} \times O}=\bigoplus_{h} \bigoplus_{a \geq 0} P_{|h|+2 a} \mathcal{G} r_{h}^{\prime}
$$

We take a holomorphic frame $\boldsymbol{u}_{h}$ of $P_{h} \mathcal{G} r_{h}^{\prime}$. Then we obtain the holomorphic frame of ${ }^{\diamond} \mathcal{E}_{\mid \mathbf{C}_{\lambda} \times O}$ given as follows:

$$
\bigcup_{h}\left(\bigcup_{a=0}^{h} \operatorname{Res}\left(\mathbb{D}^{\lambda}\right)^{a}\left(\boldsymbol{u}_{h}\right)\right) .
$$

We extend the frame to a holomorphic frame of ${ }^{\diamond} \mathcal{E}$ over $\mathbf{C}_{\lambda} \times \Delta$, which is a desired frame. q.e.d.

Let $\boldsymbol{v}$ be a holomorphic frame of ${ }^{\diamond} \mathcal{E}$ over $\mathbf{C}_{\lambda} \times \Delta$, compatible with the filtration $W$. We put $2 \cdot k\left(v_{i}\right):=\operatorname{deg}^{W}\left(v_{i}\right)$. Then we put $v_{i}^{\prime}:=$ $v_{i} \cdot(-\log |z|)^{-k\left(v_{i}\right)}$, and we obtain the $C^{\infty}$-frame $\boldsymbol{v}^{\prime}=\left(v_{i}^{\prime}\right)$ over $\mathbf{C}_{\lambda} \times \Delta^{*}$.

Proposition 4.7. For any compact subset $K$ of $\mathbf{C}_{\lambda}$, the frame $\boldsymbol{v}^{\prime}$ is adapted over $K \times \Delta^{*}$.

Proof. We can assume that $\boldsymbol{v}$ satisfies the condition in Lemma 4.17. We take a model bundle $E(V, J)=\left(E_{0}, \theta_{0}, h_{0}\right)$. We denote the deformed holomorphic bundle by $\left(\mathcal{E}_{0}, \mathbb{D}_{0}, h_{0}\right)$. We have the canonical frame $\boldsymbol{v}_{0}$ of ${ }^{\diamond} \mathcal{E}_{0}$, such that $\mathbb{D}_{0} \cdot \boldsymbol{v}_{0}=\boldsymbol{v}_{0} \cdot J \cdot d z / z$. Then the frames $\boldsymbol{v}$ and $\boldsymbol{v}_{0}$ induce the holomorphic isomorphism $\Phi:{ }^{\diamond} \mathcal{E}_{0} \longrightarrow{ }^{\diamond} \mathcal{E}$. Then we have the following:

$$
\Phi \circ \operatorname{Res}\left(\mathbb{D}_{0}\right)-\operatorname{Res}(\mathbb{D}) \circ \Phi=0, \quad \text { on } \mathbf{C}_{\lambda} \times\{O\} .
$$

We only have to show the boundedness of $\Phi$ and $\Phi^{-1}$ over $K \times \Delta^{*}$ for any compact subset $K \subset \mathbf{C}_{\lambda}$. 
Note that $\Phi$ is a holomorphic section of $\operatorname{Hom}\left(\mathcal{E}_{0}, \mathcal{E}\right)$, which is a deformed holomorphic bundle of $\left(\operatorname{Hom}\left(E_{0}, E\right),-\theta_{0} \otimes 1+1 \otimes \theta, h_{0} \otimes h\right)$. Due to the Lemma 4.18 below, we have the inequality $\Delta_{z}^{\prime \prime}|\Phi|_{h_{0} \otimes h} \leq$ $|\mathbb{D}(\Phi)|_{h_{0} \otimes h}$. Due to $(42), \mathbb{D}(\Phi)$ is holomorphic over $\mathbf{C}_{\lambda} \times \Delta$, Thus we have the following inequality over $K \times \Delta^{*}$ for any compact subset $K \subset \mathbf{C}_{\lambda}$ :

$$
\Delta_{z}^{\prime \prime}|\Phi|_{h_{0} \otimes h} \leq|\mathbb{D}(\Phi)|_{h_{0} \otimes h} \leq C \cdot r^{-1+\epsilon}
$$

Here $\Delta_{z}^{\prime \prime}=-\left(\partial_{t}^{2}+\partial_{s}^{2}\right)$ for a real coordinate $z=s+\sqrt{-1} t$. Hence we obtain the estimate, which is locally uniform for $\lambda$. q.e.d.

Lemma 4.18. Let $\left(E, \bar{\partial}_{E}, \theta, h\right)$ be a tame nilpotent harmonic bundle over $\Delta^{*}$. Let $f$ be a holomorphic section of $\mathcal{E}^{\lambda}$. Then we obtain the following inequality:

$$
\Delta_{z}^{\prime \prime}|f|_{h} \leq\left|\mathbb{D}^{\lambda} f\right|_{h}
$$

Here $\Delta_{z}^{\prime \prime}=-\left(\partial_{t}^{2}+\partial_{s}^{2}\right)$ for a real coordinate $z=s+\sqrt{-1} t$.

Proof. We denote the metric connection of $\mathcal{E}^{\lambda}$ by $d^{\prime \prime \lambda}+d^{\prime \lambda}$. We have the following equality:

$$
\partial \bar{\partial}|f|_{h}^{2}=\left(d^{\prime \lambda} f, d^{\prime \lambda} f\right)_{h}+\left(f, R\left(d^{\prime \prime \lambda}\right) f\right)_{h} .
$$

We also have the following equality:

$$
\begin{aligned}
R\left(d^{\prime \prime \lambda}\right) & =\bar{\partial}_{E} \partial_{E}+\partial_{E} \bar{\partial}_{E}-|\lambda|^{2} \cdot\left(\theta^{\dagger} \cdot \theta+\theta \cdot \theta^{\dagger}\right) \\
& =-\left(1+|\lambda|^{2}\right) \cdot\left(\theta^{\dagger} \cdot \theta+\theta \cdot \theta^{\dagger}\right) .
\end{aligned}
$$

Thus we obtain the following:

$$
\partial \bar{\partial}|f|_{h}^{2}=\left(d^{\prime \lambda} f, d^{\prime \lambda} f\right)_{h}-\left(1+|\lambda|^{2}\right) \cdot(\theta f, \theta f)_{h}-\left(1+|\lambda|^{2}\right) \cdot\left(\theta^{\dagger} f, \theta^{\dagger} f\right)_{h} .
$$

We also have the following:

$$
\begin{aligned}
& \left(d^{\prime \lambda} f, d^{\prime \lambda} f\right)_{h}+\left(\mathbb{D}^{\lambda} f, \mathbb{D}^{\lambda} f\right)_{h}-\left(1+|\lambda|^{2}\right) \cdot(\theta f, \theta f)_{h} \\
& =-\left(1+|\lambda|^{2}\right) \cdot\left(\partial_{E} f, \partial_{E} f\right)_{h} .
\end{aligned}
$$

Thus we obtain the following:

$$
\begin{aligned}
\partial \bar{\partial}|f|_{h}^{2}= & \left(1+|\lambda|^{2}\right) \cdot\left(\partial_{E} f, \partial_{E} f\right)_{h}-\left(\mathbb{D}^{\lambda} f, \mathbb{D}^{\lambda} f\right)_{h} \\
& -\left(1+|\lambda|^{2}\right) \cdot\left(\theta^{\dagger} f, \theta^{\dagger} f\right)_{h} .
\end{aligned}
$$


Then we obtain the following:

$$
\Delta^{\prime \prime}|f|_{h}^{2}=-\left(1+|\lambda|^{2}\right) \cdot\left|\partial_{E} f\right|_{h}^{2}+\left|\mathbb{D}^{\lambda} f\right|_{h}^{2}-\left(1+|\lambda|^{2}\right) \cdot\left|\theta^{\dagger} f\right|_{h}^{2} \leq\left|\mathbb{D}^{\lambda} f\right|_{h}^{2} .
$$

Thus we are done.

q.e.d.

We have a $\lambda$-family version of Lemma 4.9. Let $\boldsymbol{v}$ be a holomorphic frame of ${ }^{\diamond} \mathcal{E}$ over $\mathbf{C}_{\lambda} \times \Delta$, compatible with the filtration $W$. We have the vector space $V={ }^{\diamond} \mathcal{E}_{\mid(0, O)}$ and the endomorphism $N=\operatorname{Res}(\mathbb{D})_{\mid(0, O)}$. Then we obtain the model bundle $E(V, N)=\left(E_{0}, \theta_{0}, h_{0}\right)$. We denote the deformed holomorphic bundle by $\left(\mathcal{E}_{0}, \mathbb{D}_{0}, h_{0}\right)$. We have the canonical frame $\boldsymbol{v}_{0}$.

By the frames $\boldsymbol{v}_{\mid\{0\} \times \Delta^{*}}$ and $\boldsymbol{v}_{0 \mid\{0\} \times \Delta^{*}}$, we obtain the holomorphic isomorphism $\mathcal{I}: \mathcal{E}_{0}^{0} \longrightarrow \mathcal{E}^{0}$ over $\{0\} \times \Delta$. Then we obtain the $C^{\infty}$ isomorphism $\mathcal{I}: \mathcal{E}_{0} \longrightarrow \mathcal{E}$ defined over $\mathbf{C}_{\lambda} \times \Delta^{*}$. Then we obtain the elements $I_{i j}(\lambda, z) \in C^{\infty}\left(\mathbf{C}_{\lambda} \times \Delta_{z}^{*}\right)$, determined as follows:

$$
\mathcal{I}\left(v_{0 j}\right)=\sum I_{i j}(\lambda, z) \cdot v_{i}, \quad \mathcal{I}\left(\boldsymbol{v}_{0}\right)=\boldsymbol{v} \cdot I .
$$

Note that $I_{i j}$ are holomorphic along the direction of $\lambda$, although it is not holomorphic along the direction of $z$. The following lemma is clear from the proof of Lemma 4.9.

Lemma 4.19. The finiteness in Lemma 4.9 is locally uniform on $\mathbf{C}_{\lambda}$. Namely the values of integrals (32) and (34) are bounded on any compact subset $K \subset \mathbf{C}_{\lambda}$.

\subsection{Extension of holomorphic sections over hyperplanes}

\subsubsection{Preliminary}

For later use, we give a way of the construction of some $C^{\infty}$-section from a holomorphic section of the restriction of ${ }^{\diamond} \mathcal{E}^{0}$ to a hyperplane, which is transversal with the singularity. Let $\left(E, \bar{\partial}_{E}, \theta, h\right)$ be a tame nilpotent harmonic bundle with the trivial parabolic structure over $\Delta_{\zeta} \times$ $\Delta_{z}^{* n-1}=\left\{\left(\zeta, z_{1}, \ldots, z_{n-1}\right)\right\}$. We note that we have already known that the prolongment ${ }^{\diamond} \mathcal{E}^{1}$ is locally free (Subsubsection 4.6.1).

We put $X-D:=\Delta_{\zeta} \times \Delta_{z}^{* n-1}, X:=\Delta_{\zeta} \times \Delta_{z}^{n-1}, X_{0}-D_{0}:=$ $\{0\} \times \Delta_{z}^{* n-1}$, and $X_{0}:=\{0\} \times \Delta_{z}^{n-1}$.

Recall that we have already known the following (Lemma 4.15):

Condition 4.2. Let $\boldsymbol{v}$ be a frame of ${ }^{\diamond} \mathcal{E}^{1}$ over $\Delta_{\zeta} \times \Delta_{z}^{* n-1}$. The components of the hermitian matrices $H(h, \boldsymbol{v})$ and the inverse $H(h, \boldsymbol{v})^{-1}$ are dominated by polynomials of $-\log \left|z_{i}\right|$ for $i=1, \ldots, n-1$. 
By the relations $\theta \cdot \boldsymbol{v}=\boldsymbol{v} \cdot \Theta$ or $\theta^{\dagger} \cdot \boldsymbol{v}=\boldsymbol{v} \cdot \Theta^{\dagger}$, we have the following elements:

$$
\begin{aligned}
& \Theta=\Theta^{\zeta} \cdot d \zeta+\sum \Theta^{k} \cdot d z_{k} / z_{k} \in C^{\infty}\left(X, M(r) \otimes \Omega_{X-D}^{1,0}\right) \\
& \Theta^{\dagger}=\Theta^{\zeta \dagger} \cdot d \bar{\zeta}+\sum \Theta^{k \dagger} \cdot d \bar{z}_{k} / \bar{z}_{k} \in C^{\infty}\left(X, M(r) \otimes \Omega_{X-D}^{0,1}\right) .
\end{aligned}
$$

By the estimate for $\theta$ (Proposition 4.1), the absolute values of the components of $\Theta^{\zeta}, \Theta^{k}, \Theta^{\zeta \dagger}$ and $\Theta^{k \dagger}$ are bounded by the polynomials of $-\log \left|z_{i}\right|$ for $i=1, \ldots, n-1$.

We have the flat connection form $A$ of $\mathbb{D}^{1}$ with respect to the frame $\boldsymbol{v}$, that is, $\mathbb{D}^{1} \boldsymbol{v}=\left(\partial_{E}+\theta\right) \boldsymbol{v}=\boldsymbol{v} \cdot A$. The form $A$ is a holomorphic section of $M(r) \otimes \Omega_{X}^{1,0}(\log D)$.

We have the relation $\partial_{E}\left(\theta^{\dagger}\right)=0$. It is translated as follows:

$$
\partial \Theta^{\dagger}+\left[A-\Theta, \Theta^{\dagger}\right]=0 .
$$

\section{Lemma 4.20.}

- The components of $\partial_{\zeta} \Theta^{\dagger}$ is of the form $\sum_{k} C_{k} \cdot d \bar{z}_{k} / \bar{z}_{k} \cdot d \zeta+C_{\zeta}$. $d \bar{\zeta} \cdot d \zeta$. Then the absolute values of $C_{k}$ and $C_{\zeta}$ are dominated by a polynomial of $\left(-\log \left|z_{i}\right|\right)$ for $i=1, \ldots, n-1$.

- The components of $\partial_{k} \Theta^{\zeta \dagger}$ is of the form $C \cdot d \bar{z}_{k} / \bar{z}_{k} \cdot d \bar{\zeta}$. Then the absolute values of $C$ is dominated by a polynomials of $\left(-\log \left|z_{i}\right|\right)$ for $i=1, \ldots, n-1$.

- Hence, for any $z=\left(z_{1}, \ldots, z_{n-1}\right) \in X_{0}, \Theta^{\dagger}(\zeta, z)$ is $L_{k}^{p}$ as a function of $\zeta$. And the $L_{k}^{p}$-norm is dominated by the polynomials of $\left(-\log \left|z_{i}\right|\right)$ for $i=1, \ldots, n-1$.

Proof. The claims immediately follow from (43).

q.e.d.

The restriction of the frame $\boldsymbol{v}$ to $\mathcal{E}^{1}$ over $X-D$ can be regarded as a $C^{\infty}$-frame of $\mathcal{E}^{0}$. Let $f$ be a holomorphic section of $\diamond^{\circ}\left(\mathcal{E}_{\mid X_{0}-D_{0}}^{0}\right)$. We have $C^{\infty}$-functions $f_{i}$ on $X_{0}-D_{0}$ determined by the relation:

$$
f(z)=\sum_{i} f_{i}(z) \cdot v_{i}(0, z)
$$

Since an increasing order of $f$ is less than 0 , we have some inequalities $|f|_{h} \leq C_{\epsilon} \cdot\left(\prod_{j=1}^{l}\left|z_{j}\right|\right)^{-\epsilon}$ for any $\epsilon>0$. Due to Condition 4.2 , we have 
some inequalities $\left|f_{i}\right|_{h} \leq C_{\epsilon} \cdot\left(\prod_{j=1}^{l}\left|z_{j}\right|\right)^{-\epsilon}$ for any $\epsilon>0$. Since $f$ is holomorphic, we have the following equality:

$$
0=\bar{\partial} f(z)=\sum\left(\bar{\partial} f_{i}(z)+\sum_{k} \Theta_{i j}^{k \dagger}(0, z) \cdot \bar{\eta}_{k} f_{j}(z)\right) \cdot v_{i}(0, z) .
$$

Here we use the notation $\bar{\eta}_{i}=d \bar{z}_{i} / \bar{z}_{i}$.

Let $\mathcal{O}\left(-X_{0}\right)$ denote the sheaf of holomorphic functions which vanish on the divisor $X_{0}$. It is the line bundle over $X$. The restriction of $\mathcal{O}\left(-X_{0}\right)$ is denoted by the same notation. We put $\mathcal{E}^{0}\left(-X_{0}\right):=\mathcal{E}^{0} \otimes$ $\mathcal{O}\left(-X_{0}\right)$.

Lemma 4.21. There exists a $C^{\infty}$-function $\rho$ defined over $X-D$ satisfying the following:

1. $\rho$ is an element of $A_{-\epsilon \cdot \boldsymbol{\delta}, N}^{0,0}\left(\mathcal{E}^{0}\right)$.

2. $\bar{\partial}_{E}(\rho)$ is an element of $A_{-\epsilon \cdot \boldsymbol{\delta}, N}^{0,1}\left(\mathcal{E}^{0}\left(-X_{0}\right)\right)$.

3. We have $\rho_{\mid X_{0}-D_{0}}=f$.

4. The restriction of $\rho$ to $\{\zeta|| \zeta \mid>2 / 3\} \times \Delta^{* n-1}$ vanishes identically.

Proof. Take a cut function $\chi$ on $\Delta_{\zeta}$ satisfying $\chi(\zeta)=1$ for $|\zeta|<1 / 3$ and $\chi(\zeta)=0$ for $|\zeta|>2 / 3$. We put as follows:

$$
\begin{aligned}
f^{1} & :=\sum_{i} f_{i}(z) \cdot v_{i}(\zeta, z), \quad f^{2}:=\bar{\zeta} \cdot g^{2}, \\
g^{2} & :=\sum_{i, j} \Theta_{i j}^{\zeta \dagger}(0, z) \cdot f_{j}(z) \cdot v_{i}(\zeta, z) .
\end{aligned}
$$

Then we put $\rho:=\chi \cdot\left(f_{1}-f_{2}\right)$. Then Conditions 3 and 4 are satisfied.

We have the following equality:

$$
\begin{aligned}
\bar{\partial} f^{1}= & \sum\left(\bar{\partial} f_{i}(z)+\left(\sum_{k} \Theta_{i j}^{k \dagger}(\zeta, z) \bar{\eta}_{k}+\Theta_{i j}^{\zeta \dagger}(\zeta, z) d \bar{\zeta}\right) \cdot f_{j}\right) v_{i}(\zeta, z) \\
= & \sum\left(\sum_{k}\left(\Theta_{i j}^{k \dagger}(\zeta, z)-\Theta_{i j}^{k \dagger}(0, z)\right) \cdot \bar{\eta}_{k}\right) \cdot f_{j}(z) \cdot v_{i}(\zeta, z) \\
& +\sum \Theta_{i j}^{\zeta \dagger}(\zeta, z) \cdot d \bar{\zeta} \cdot f_{j}(z) \cdot v_{i}(\zeta, z) .
\end{aligned}
$$

We also have the following equality:

$$
\bar{\partial} f^{2}=\sum \Theta_{i j}^{\zeta \dagger}(0, z) \cdot d \bar{\zeta} \cdot f_{j}(z) \cdot v_{i}(\zeta, z)+\bar{\zeta} \cdot \bar{\partial} g^{2} .
$$


Thus we obtain the equality $\bar{\partial} \rho=\zeta \cdot\left(F_{1}+F_{2}\right)$, when we put as follows:

$$
\begin{aligned}
F_{1}:=\zeta^{-1} \cdot \bar{\partial} \chi \cdot\left(f_{1}-f_{2}\right) \\
F_{2}:=\zeta^{-1} \cdot \chi \cdot\left[\sum \left(\sum_{k}\left(\Theta_{i j}^{k \dagger}(\zeta, z)-\Theta_{i j}^{k \dagger}(0, z)\right) d \bar{z}_{k}\right.\right. \\
\left.\left.\quad+\left(\Theta_{i j}^{\zeta \dagger}(\zeta, z)-\Theta_{i j}^{\zeta \dagger}(0, z)\right) d \bar{\zeta}\right) \cdot f_{j} \cdot v_{i}(\zeta, z)+\bar{\zeta} \cdot \bar{\partial} g^{2}\right] .
\end{aligned}
$$

By our construction, the norms $\left|F_{i}\right|_{h, g_{\mathbf{p}}}$ are dominated by polynomials of $\left(-\log \left|z_{i}\right|\right)$ for $i=1, \ldots, l$. (Use Lemma 4.20.) Thus we have the finiteness for any $\epsilon>0$ and any number $M$ and $i=1,2$ :

$$
\int\left|F_{i}\right|_{h}^{2} \cdot \prod_{j=1}^{n-1}\left(\left|z_{j}\right|^{\epsilon} \cdot\left(-\log \left|z_{j}\right|\right)^{M}\right) \mathrm{dvol}<\infty .
$$

Here dvol is obtained from the Poincaré metric. Hence we obtain Condition 2. Then Condition 1 is easy.

q.e.d.

\subsubsection{Extension of holomorphic sections, statement}

We put $X=\Delta^{n}=\left\{\left(z_{1}, \ldots, z_{n}\right)\right\}$ and $D=\bigcup_{i=1}^{l} D_{i}$, where $D_{i}:=\left\{z_{i}=\right.$ $0\}$. We put $X^{(1)}:=\left\{\left(z_{1}, \ldots, z_{n}\right) \in X \mid z_{1}=z_{2}\right\}$ and $D^{(1)}:=X^{(1)} \cap D$. We put $X_{0}=\left\{\left(z_{1}, \ldots, z_{n}\right) \in X \mid z_{1}=z_{2}=0\right\}$. Let $\left(E, \bar{\partial}_{E}, \theta, h\right)$ be a tame nilpotent harmonic bundle with trivial parabolic structure over $X-D$.

We will prove the following proposition in the next subsubsections.

Proposition 4.8. Let $f$ be a holomorphic section of ${ }^{\diamond} \mathcal{E}_{\mid X^{(1)}-D^{(1)}}^{0}$ on $X^{(1)}$. Then there exist a neighborhood $U$ of $X_{0}$ in $X$ and a holomorphic section $\widetilde{f}$ of $\diamond \mathcal{E}^{0}$ over $U$ satisfying $\widetilde{f}_{\mid X^{(1)} \cap U}=f_{\mid X^{(1)} \cap U}$.

We reword the proposition as follows: We put $\Delta_{z}^{2}=\left\{\left(z_{1}, z_{2}\right)|| z_{i} \mid<\right.$ $1\}$ and $D_{i}^{\prime}=\left\{z_{i}=0\right\} \subset \Delta_{z}^{2}$. Let $\varphi: \widetilde{\Delta_{z}^{2}} \longrightarrow \Delta_{z}^{2}$ denote the blow up of $\Delta_{z}^{2}$ at the origin $O=(0,0)$. We have the exceptional divisor $\varphi^{-1}(O)$, the proper transforms $\widetilde{D^{\prime}}{ }_{i}$ of $D_{i}^{\prime}$.

We put $\widetilde{X}=\widetilde{\Delta_{z}^{2}} \times \Delta_{w}^{n-2}$. Then we have the composite $\psi$ of the natural morphisms:

$$
\widetilde{X} \stackrel{\varphi \times \mathrm{id}}{\longrightarrow} \Delta_{z}^{2} \times \Delta_{w}^{n-2} \longrightarrow \Delta_{z}^{n}
$$


Here the latter morphism is the natural isomorphism given by $w_{i}=z_{i+2}$. We put $\widetilde{D}:=\psi^{-1}(D)$, which is same as the following:

$$
\left(\varphi^{-1}(0,0) \cup \widetilde{D_{1}^{\prime}} \cup \widetilde{D_{2}^{\prime}}\right) \times \Delta_{w}^{n-2} \cup \Delta_{z}^{2} \times\left(\bigcup_{i=1}^{n-2}\left\{w_{i}=0\right\}\right) .
$$

The restriction of $\psi$ to $\widetilde{X}-\widetilde{D}$ gives an isomorphism $\widetilde{X}-\widetilde{D} \simeq X-D$.

We have the diagonal $C(1,1):=\left\{(z, z) \in \Delta^{2}\right\}$ of $\Delta^{2}$, and the proper transform $\widetilde{C}(1,1) \subset \widetilde{\Delta^{2}}$. We put $\widetilde{X^{(1)}}:=\widetilde{C}(1,1) \times \Delta_{w}^{n-2}$ and $\widetilde{D^{(1)}}:=$ $\widetilde{X^{(1)}} \cap \widetilde{D}$. The restriction $\psi$ to $\widetilde{X^{(1)}}$ gives an isomorphism $\widetilde{X^{(1)}} \simeq X^{(1)}$.

We also put $\widetilde{X}_{0}:=\varphi^{-1}(0,0) \times \Delta_{w}^{n-2}=\psi^{-1}\left(X_{0}\right)$.

Proposition 4.8 is equivalent to the following lemma.

Lemma 4.22. Let $f$ be a holomorphic section of $\diamond\left(\psi^{*} \mathcal{E}_{\mid \widetilde{X^{(1)}}}^{0}-\widetilde{D^{(1)}}\right)$ over $\widetilde{X^{(1)}}$. Then there exist a neighborhood $\widetilde{U}$ of $\widetilde{X_{0}}$ in $\widetilde{X}$ and a holomorphic section $\tilde{f}$ of ${ }^{\diamond} \psi^{*} \mathcal{E}^{0}$ over $\widetilde{U}$ satisfying $\widetilde{f}_{\mid \widetilde{X^{(1)}} \cap U}=f_{\mid \widetilde{X^{(1)} \cap}}$.

Let us see the equivalence of Proposition 4.8 and Lemma 4.22. First we remark that neighborhoods of $X_{0}$ and $\widetilde{X}_{0}$ correspond bijectively. Since $\widetilde{X}-\widetilde{D}$ and $X-D$ are isomorphic via $\psi$, we have the following:

$$
\Gamma\left(\widetilde{U} \cap(\widetilde{X}-\widetilde{D}), \psi^{*} \mathcal{E}^{0}\right)=\Gamma\left(U \cap(X-D), \mathcal{E}^{0}\right)=: \mathcal{H} .
$$

The spaces $\Gamma\left(\widetilde{U},{ }^{\diamond} \psi^{*} \mathcal{E}^{0}\right)$ and $\Gamma\left(U,{ }^{\diamond} \mathcal{E}^{0}\right)$ are defined as the subspaces of $\mathcal{H}$. It is easy to see that the conditions are equivalent. Thus the equivalence of Proposition 4.8 and Lemma 4.22 are shown.

In the next subsubsections, we will show Proposition 4.8 or equivalently, Lemma 4.22 .

\subsubsection{The metrics and the curvatures of $\mathcal{O}(i)$ on $\mathbb{P}^{1}$}

Before entering the proof of Lemma 4.22, we need some preparation on the line bundles over $\mathbb{P}^{1}$. We denote the one dimensional projective space over $\mathbf{C}$ by $\mathbb{P}^{1}=\left\{\left[t_{0}: t_{1}\right]\right\}$. The points $[0: 1]$ and $[1: 0]$ are denoted by 0 and $\infty$ respectively. We use the coordinates $t=t_{0} / t_{1}$ and $s=t_{1} / t_{0}$. We have the line bundle $\mathcal{O}(i)$ over $\mathbb{P}^{1}$. The coordinates of $\mathcal{O}(i)$ is given as follows: $\left(t, \zeta_{1}\right)$ over $\mathbb{P}^{1}-\{\infty\}$, and $\left(s, \zeta_{2}\right)$ over $\mathbb{P}^{1}-\{0\}$. The relations are given by $s=t^{-1}$ and $t^{-i} \cdot \zeta_{1}=\zeta_{2}$.

Recall that we have the smooth metric $h_{i}$ of $\mathcal{O}(i)$. Let $\xi=\left(t, \zeta_{1}\right)=$ $\left(s, \zeta_{2}\right)$ be an element of $\mathcal{O}(i)$.

$$
h_{i}(\xi, \xi):=\left|\zeta_{1}\right|^{2} \cdot\left(1+|t|^{2}\right)^{-i}=\left|\zeta_{2}\right|^{2} \cdot\left(1+|s|^{2}\right)^{-i} .
$$


For any real numbers $a$ and $b$, we have the possibly singular metrics $h_{i,(a, b)}$ of $\mathcal{O}(i)$ : Let $\xi=\left(t, \zeta_{1}\right)=\left(s, \zeta_{2}\right)$ be an element of $\mathcal{O}(i)$.

$$
\begin{aligned}
h_{i,(a, b)}(\xi, \xi): & =h_{i}(\xi, \xi) \cdot\left(1+|t|^{-2}\right)^{a} \cdot\left(1+|t|^{2}\right)^{b} \\
& =h_{i}(\xi, \xi) \cdot\left(1+|s|^{2}\right)^{a} \cdot\left(1+|s|^{-2}\right)^{b} .
\end{aligned}
$$

Around $|t|=0$, the order of $h_{0,(a, b)}$ is equivalent to $|t|^{-2 a}$. Around $|s|=$ 0 , the order of $h_{0,(a, b)}$ is equivalent to $|s|^{-2 b}$. The curvature $R\left(h_{i, a, b}\right)$ is as follows:

$$
R\left(h_{i, a, b}\right)=(-a-b+i) \cdot \frac{d t \cdot d \bar{t}}{(1+|t|)^{2}} .
$$

When $i=-1$ and $a=b=-1 / 2$, then we have the following:

$$
R\left(h_{-1, a, b}\right)=0 .
$$

Take a point $P \in \mathbb{P}^{1}$. Then we obtain a morphism $\mathcal{O}(i) \longrightarrow \mathcal{O}(i+1)$ of coherent sheaves. The morphism is bounded with respect to the metrics $h_{i,(a, b)}$ and $h_{i+1,(a, b)}$.

We are mainly interested in the case $i=-1$. We regard $\mathcal{O}(-1)$ as a complex manifold. The open submanifold $Y$ is defined to be $\{\xi \in$ $\left.\mathcal{O}(-1) \mid h_{-1,(0,0)}(\xi, \xi)<1\right\}$. We denote the naturally defined projection of $Y$ onto $\mathbb{P}^{1}$ by $\pi$. We denote the image of the 0 -section $\mathbb{P}^{1} \longrightarrow Y$ by $\mathbb{P}^{1}$. Then we have the normal crossing divisor $D^{\prime}=\mathbb{P}^{1} \cup \pi^{-1}(0) \cup \pi^{-1}(\infty)$ of $Y$. The manifold $Y-D^{\prime}$ is same as $\left\{\left.(t, x) \in \mathbf{C}^{* 2}|| x\right|^{2}\left(1+|t|^{2}\right)<1\right\}$. We have the complete Kahler metric $g:=g_{1}+g_{2}+g_{3}$ of $Y-D^{\prime}$ given as follows: As a contribution of the 0 -section $\mathbb{P}^{1}$, we put $\tau_{1}=-\log [(1+$ $\left.\left.|t|^{2}\right) \cdot|x|^{2}\right]$, and as follows:

$$
g_{1}:=\frac{1}{\tau_{1}^{2}}\left(\frac{\bar{t} \cdot d t}{1+|t|^{2}}+\frac{d x}{x}\right) \cdot\left(\frac{t \cdot d \bar{t}}{1+|t|^{2}}+\frac{d \bar{x}}{\bar{x}}\right)+\frac{1}{\tau_{1}} \frac{d t \cdot d \bar{t}}{\left(1+|t|^{2}\right)^{2}} .
$$

Note that $g_{1}$ gives the complete Kahler metric of $Y-\mathbb{P}^{1}$.

As a contribution of $\pi^{-1}(\infty)$, we put $\tau_{2}=\log \left(1+|t|^{2}\right)$, and as follows:

$$
g_{2}=\frac{1}{\tau_{2}}\left(-1+\frac{|t|^{2}}{\tau_{2}}\right) \cdot \frac{d t \cdot d \bar{t}}{\left(1+|t|^{2}\right)^{2}} .
$$


Note that we have $-1+|t|^{2} \cdot \tau_{2}^{-1}>0$. Around $|t|=\infty$, or equivalently, around $|s|=0$, the $g_{2}$ is similar to $(-|s| \log |s|)^{-2} d s \cdot d \bar{s}$. Around $|t|=0$, we have $g_{2}=\left(2^{-1}+o\left(|t|^{2}\right)\right) \cdot d t \cdot d \bar{t}$.

As the contribution of the divisor $\pi^{-1}(0)$, we put $\tau_{3}:=\log \left(1+|t|^{2}\right)-$ $\log |t|^{2}=\log \left(1+|s|^{2}\right)$, where we use $s=t^{-1}$. And we put as follows:

$$
g_{3}=\frac{1}{\tau_{3}} \cdot\left(-1+\frac{|s|^{2}}{\tau_{3}}\right) \frac{d s \cdot d \bar{s}}{\left(1+|s|^{2}\right)^{2}} .
$$

By the symmetry, the behaviour of $g_{3}$ is similar to $g_{2}$.

The following lemma is easy to see.

Lemma 4.23. $g$ gives the complete Kahler metric of $Y-D^{\prime}$. Around the 0 -section $\mathbb{P}^{1}, \pi^{-1}(0)$ and $\pi^{-1}(\infty)$, the behaviours of the metric are equivalent to the Poincaré metric.

We note the following formulas:

$$
\begin{aligned}
\bar{\partial} \partial \log \tau_{1}= & \frac{1}{\tau_{1}^{2}}\left(\frac{\bar{t} \cdot d t}{1+|t|^{2}}+\frac{d x}{x}\right) \wedge\left(\frac{t \cdot d \bar{t}}{1+|t|^{2}}+\frac{d \bar{x}}{\bar{x}}\right) \\
& +\frac{1}{\tau_{1}} \frac{d t \wedge d \bar{t}}{\left(1+|t|^{2}\right)^{2}}=: \omega_{1}, \\
& \bar{\partial} \partial \log \tau_{2}=\frac{1}{\tau_{2}}\left(-1+\frac{|t|^{2}}{\tau_{2}}\right) \cdot \frac{d t \wedge d \bar{t}}{\left(1+|t|^{2}\right)^{2}}=: \omega_{2}, \\
& \bar{\partial} \partial \log \tau_{3}=\frac{1}{\tau_{3}}\left(-1+\frac{|s|^{2}}{\tau_{3}}\right) \cdot \frac{d s \wedge d \bar{s}}{\left(1+|s|^{2}\right)^{2}}=: \omega_{3} .
\end{aligned}
$$

We put $\omega=\omega_{1}+\omega_{2}+\omega_{3}$. We put as follows:

$$
H_{0}=\frac{1}{\tau_{1}}+\frac{1}{\tau_{2}}\left(-1+\frac{|t|^{2}}{\tau_{2}}\right)+\frac{1}{\tau_{3}}\left(-1+\frac{|s|^{2}}{\tau_{3}}\right)>0 .
$$

Then we have the following:

$$
\begin{aligned}
\omega^{2} & =\operatorname{det}(g) \cdot d t \wedge d \bar{t} \wedge d x \wedge d \bar{x} \\
& =\left(\frac{1}{\tau_{1}^{2} \cdot|x|^{2} \cdot\left(1+|t|^{2}\right)^{2}} \times H_{0}\right) \cdot d t \wedge d \bar{t} \wedge d x \wedge d \bar{x}
\end{aligned}
$$

We put as follows:

$$
H_{1}:=\frac{H_{0}}{\left(1+|t|^{2}\right) \cdot\left(1+|s|^{2}\right)} .
$$

Recall that we have $\operatorname{Ric}(g)=\bar{\partial} \partial(\operatorname{det}(g))$. 


\section{Lemma 4.24.}

- Let $C$ be a number such that $0<C<1$. On the domain $\{(t, x) \in$ $\left.\left.\mathbf{C}^{* 2}|| x\right|^{2} \cdot\left(1+|t|^{2}\right) \leq C\right\}$, we have the following similarity of the behaviour:

$$
H_{1} \sim(\log |t|)^{-2}, \quad(|t| \rightarrow \infty, \text { or },|t| \rightarrow 0) .
$$

- We have the equality: Ric $(g)-\bar{\partial} \partial \log \left(H_{1}\right)=-\bar{\partial} \partial \log \tau_{1}^{2}$.

\subsubsection{Proof of Proposition 4.8}

Consider the blow up $\widetilde{\Delta_{z}^{2}} \longrightarrow \Delta_{z}^{2}=\left\{\left(z_{1}, z_{2}\right)\right\}$ at $O=(0,0)$ as in Subsubsection 4.7.2. We can take a holomorphic embedding $\iota$ of $Y$, given in the previous subsubsection, to $\widetilde{\Delta}^{2}$ satisfying the following:

- The image of the 0 -section $\mathbb{P}^{1}$ is the exceptional divisor $\phi^{-1}(O)$.

- We have $\iota^{-1}\left(D_{1}^{\prime}\right)=\pi^{-1}(\infty)$ and $\iota^{-1}\left(D_{2}^{\prime}\right)=\pi^{-1}(0)$.

We may assume that $\pi^{-1}(P)=\iota^{-1}(\widetilde{C}(1,1))$ for $P=[1: 1] \in \mathbb{P}^{1}$.

We put $\bar{X}:=Y \times \Delta_{w}^{n-2}$. Then we have the naturally induced morphism $\bar{X} \longrightarrow \widetilde{\Delta_{z}^{2}} \times \Delta_{w}^{n-2}$, which we also denote by $\iota$. We put as follows:

$$
\begin{array}{rlrl}
\bar{D}:=\iota^{-1}(\widetilde{D}), & \overline{X^{(1)}}:=\iota^{-1}\left(\widetilde{X^{(1)}}\right)=\pi^{-1}(P) \times \Delta_{w}^{n-2}, \\
\overline{D^{(1)}}:=\overline{X^{(1)}} \cap \bar{D} .
\end{array}
$$

Note that $\iota(\bar{X})$ gives a neighborhood of $\widetilde{X_{0}}$ in $\widetilde{X}$. The composite $\psi \circ \iota$ is denoted by $\psi_{1}$.

Let $\left(E, \bar{\partial}_{E}, \theta, h\right)$ be a tame nilpotent harmonic bundle with trivial parabolic structure over $X-D$. We denote the curvature of $\psi_{1}^{*}\left(E, \bar{\partial}_{E}, h\right)$ by $\psi_{1}^{*} R(h)$. It is dominated by $\psi_{1}^{*} \bar{\partial} \partial \log \tau(\boldsymbol{a}, N)$ for sufficiently negative number $N$.

Let $\epsilon$ be sufficiently small positive number. The metric $\widetilde{h}$ of $\psi_{1}^{*}(E) \otimes$ $\mathcal{O}_{\mathbb{P}}(-1)$ over $Y-D^{\prime}$ is defined as follows:

$$
\widetilde{h}:=\psi_{1}^{*} h_{\boldsymbol{a}, N} \cdot h_{-1,-1 / 2,-1 / 2} \cdot H_{1}^{-1} \cdot \tau_{1}^{2+\epsilon}\left(\tau_{2} \cdot \tau_{3}\right)^{\epsilon} .
$$

Lemma 4.25. When $N$ is sufficiently smaller than 0 , then the following inequality holds for any $\eta \in A_{c}^{0,1}\left(\psi_{1}^{*} E\right)$ :

$$
\langle\langle R(\widetilde{h})+\operatorname{Ric}(g), \eta\rangle\rangle_{\widetilde{h}} \geq \epsilon\|\eta\|_{\widetilde{h}}^{2} .
$$


Proof. We have the following equality:

$$
\begin{aligned}
R(\widetilde{h})+\operatorname{Ric}(g)= & R\left(\psi_{1}^{*} h_{\boldsymbol{a}, N}\right)+R\left(h_{-1,-1 / 2,-1 / 2}\right)-\bar{\partial} \partial \log H_{1} \\
& +(2+\epsilon) \cdot \bar{\partial} \partial \log \tau_{1}+\epsilon \cdot \bar{\partial} \partial\left(\log \tau_{2}+\log \tau_{3}\right)+\operatorname{Ric}(g) \\
= & R\left(\psi_{1}^{*} h_{\boldsymbol{a}, N}\right)+\epsilon\left(\omega_{1}+\omega_{2}+\omega_{3}\right) .
\end{aligned}
$$

By taking sufficiently negative $N$, we can assume the following inequality for any $\eta \in A_{c}^{0,1}(E)$ on $X-D$ :

$$
\left\langle\left\langle R\left(h_{\boldsymbol{a}, N}\right), \eta\right\rangle\right\rangle_{\boldsymbol{a}, N} \geq 0 .
$$

Then, by a fiberwise linear algebraic argument, it is easy to see that the following inequality holds for any $\eta \in A_{c}^{0,1}\left(\psi_{1}^{*}(E)\right)$ :

$$
\left\langle\left\langle\psi^{*} R\left(h_{\boldsymbol{a}, N}\right), \eta\right\rangle\right\rangle_{\tilde{h}} \geq 0 .
$$

On the other hand, we obtain $\left\langle\left\langle\omega_{1}+\omega_{2}+\omega_{3}, \eta\right\rangle\right\rangle_{\widetilde{h}} \geq \epsilon \cdot\|\eta\|_{\tilde{h}}$, which can be checked directly from definition. Thus we are done. q.e.d.

Let $f$ be a holomorphic section of $\diamond^{\diamond}\left(\mathcal{E}_{\mid X_{0}-D_{0}}^{0}\right)$, or equivalently, ${ }^{\diamond} \psi^{*} \mathcal{E}_{\mid \widetilde{X}_{0}-\widetilde{D}_{0}}^{0}$. Clearly Lemma 4.22 can be reduced to the following lemma.

Lemma 4.26. There exists a holomorphic section $\tilde{f}$ of ${ }^{\diamond} \psi_{1}^{*} \mathcal{E}^{0}$ over $\overline{X^{(1)}}$ such that $\tilde{f}_{\mid \bar{X}^{(1)}}=f_{\mid \bar{X}^{(1)}}$.

Proof. Take an embedding $\kappa: \Delta_{\zeta} \longrightarrow \mathbb{P}^{1}-\{0, \infty\}$ such that $\kappa(0)=$ $P$ By using Lemma 4.21, we can take a $C^{\infty}$-function $\rho$ whose support is contained in $\pi^{-1}\left(\kappa\left(\Delta_{\zeta}\right)\right)$, and satisfying the following:

- $\rho$ is an element of $A_{\tilde{h}}^{0,0}\left(\psi_{1}^{*} \mathcal{E}^{0}\right)$.

- $\bar{\partial}_{E}(\rho)$ is an element of $A_{\widetilde{h}}^{0,1}\left(\psi_{1}^{*} \mathcal{E}^{0}\left(-\overline{X^{(1)}}\right)\right)$.

- We have $\rho_{\mid \bar{X}^{(1)}-\bar{D}^{(1)}}=f$.

Here $\psi_{1}^{*} \mathcal{E}^{0}\left(-\overline{X^{(1)}}\right)$ is same as $\psi_{1}^{*} \mathcal{E}^{0} \otimes \mathcal{O}_{\mathbb{P}^{1}}(-1)$.

Due to Proposition 4.3, Proposition 4.4, Corollary 4.11 and Lemma 4.25 , we can pick an element $G$ of $A_{\widetilde{h}}^{0,0}\left(\psi_{1}^{*} \mathcal{E}^{0}\left(-\overline{X^{(1)}}\right)\right)$ such that $\bar{\partial} G=\bar{\partial} \rho$. Then we put $\tilde{f}:=\rho-G$. Then it satisfies $\bar{\partial} \tilde{f}=0, \tilde{f} \in$ $A_{\widetilde{h}}^{0,0}\left(\psi_{1}^{*} \mathcal{E}^{0}\right)$, and $\widetilde{f}_{\mid \bar{X}^{(1)}-\bar{D}^{(1)}}=f_{\mid \bar{X}^{(1)}-\bar{D}^{(1)}}$. 
Let us check that $\tilde{f}$ gives a section of ${ }^{\diamond} \psi_{1}^{*} \mathcal{E}^{0}$. We regard $\tilde{f}$ as a section of $\mathcal{E}$ over an open subset $\psi_{1}(\bar{X}-\bar{D})$ of $X-D$. We only have to check that $\tilde{f}$ gives a section of ${ }^{\diamond} \mathcal{E}^{0}$ over $\psi_{1}(\bar{X})$. To show it, consider the norm of $\widetilde{f}$ with respect to our original metric $h$. We consider the restriction of $\widetilde{f}$ to $\pi_{j}^{-1}(a)$, for any $a \in \Delta^{* l-1} \times \Delta^{n-l}$ and for $1 \leq j \leq l$. First we consider the case $j=1$. On $\pi_{1}^{-1}(a)$, the metric $\widetilde{h}$ is equivalent to $h \cdot\left|z_{1}\right|^{1 / 2+\epsilon} \times Q$, where $Q$ denotes a polynomial of $\log \left|z_{1}\right|$. Thus the increasing order of $\left|\widetilde{f}_{\mid \pi_{1}^{-1}(a)}\right|_{h}$ is less than $-1 / 2-\epsilon$. Since the parabolic structure is trivial, we can conclude that the increasing order is, in fact, less than 0 . Similarly we can show that the increasing order of $\widetilde{f}_{\mid \pi_{2}^{-1}(a)}$ with respect to the metric $h$ is 0 . If $j>2$, then the metrics $h$ and $\tilde{h}$ are equivalent on $\pi_{j}^{-1}(a)$. Thus the increasing order of $\widetilde{f}_{\mid \pi_{j}^{-1}(a)}$ with respect to the metric $h$, is less than 0 , also in this case. Thus we obtain that $\tilde{f}$ is, in fact, a section of ${ }^{*} \psi_{1}^{*} \mathcal{E}^{0}$, due to Corollary 4.12 . $\quad$ q.e.d.

\subsubsection{Local freeness of prolongments}

We put $X=\Delta^{n}=\left\{\left(z_{1}, \ldots, z_{n}\right)\right\}$ and $D=\bigcup_{i=1}^{l} D_{i}$, where $D_{i}:=$ $\left\{z_{i}=0\right\}$. We put $X^{(1)}:=\left\{\left(z_{1}, \ldots, z_{n}\right) \in X \mid z_{1}=z_{2}\right\}$ and $D^{(1)}:=$ $X^{(1)} \cap D$. Let $\left(E, \bar{\partial}_{E}, \theta, h\right)$ be a tame nilpotent harmonic bundle with trivial parabolic structure over $X$.

The following theorem is an easy corollary of Proposition 4.8.

Theorem 4.1. The prolongments ${ }^{\diamond} \mathcal{E}^{0}$ and ${ }^{\diamond} \mathcal{E}$ are locally free.

Proof. We only have to show that ${ }^{\diamond} \mathcal{E}^{0}$ is locally free, due to Proposition 4.6. To show the local freeness of $\diamond^{0}$, we use an induction of the dimension $n$ of base manifolds. We assume that the claim holds for $n-1$. Then ${ }^{\diamond} \mathcal{E}_{\mid X^{(1)}-D^{(1)}}^{0}$ is locally free. Let $\boldsymbol{v}=\left(v_{i}\right)$ be a holomorphic frame of $\diamond\left(\mathcal{E}_{\mid C(1,1) \times \Delta^{n-2}}^{0}\right)$. We can extend $v_{i}$ to a section $\widetilde{v}_{i}$ of $\diamond^{0}$ due to Proposition 4.8.

Then we obtain the tuple of holomorphic sections $\widetilde{\boldsymbol{v}}=\left(\widetilde{v}_{i}\right)$. By the same argument as the proof of Proposition 4.6, we can show that $\mathcal{E}^{0}$ is locally free and that $\widetilde{\boldsymbol{v}}$ gives a local frame. Thus the induction can proceed.

q.e.d.

\section{Proposition 4.9.}

- For any $\lambda \in \mathbf{C}, \mathbb{D}^{\lambda}$ is of log type. Namely if $f$ is a holomorphic section of ${ }^{\diamond} \mathcal{E}^{\lambda}$, then $\mathbb{D}^{\lambda}(f)$ is a holomorphic section of $\mathcal{E}^{\lambda} \otimes$ $\Omega_{\mathcal{X}^{\lambda}}^{1,0}\left(\log \mathcal{D}^{\lambda}\right)$ 
- $\mathbb{D}$ is of log type.

Proof. If $\lambda \neq 0$, a normalizing frame gives a trivialization of ${ }^{\diamond} \mathcal{E}^{\lambda}$. It implies that $\mathbb{D}^{\lambda}$ is of log type. If $\lambda=0$, we know the estimate of the norm of $\theta$. It implies that $\theta$ is of $\log$ type.

Let $f$ be a holomorphic section of $\diamond \mathcal{E}$. The section $\mathbb{D}(f)$ is holomorphic over $\mathcal{X}^{\sharp} \cup(\mathcal{X}-\mathcal{D})=\mathcal{X}-\mathcal{D}^{0}$. Thus it naturally gives a holomorphic section of $\diamond \mathcal{E} \otimes p_{\lambda}^{*} \Omega_{X}(\log D)$. $\quad$ q.e.d.

Remark 4.7. Note that we also obtain the extendability of the holomorphic sections over the curves. We will use the fact without mention.

Remark 4.8. It is rather easy to see that ${ }^{\diamond} \mathcal{E}^{0}$ is locally free in codimension one. We only have to use the construction in Subsubsection 4.7.1, the arguments in the proofs of Lemma 4.16 and Proposition 4.6.

It is interesting that we can derive the local freeness of ${ }^{\diamond} \mathcal{E}^{0}$ from the codimension one result above, if the dimension of the base complex manifold is 2. Consider the case $X=\Delta^{2}=\left\{\left(z_{1}, z_{2}\right)\right\}$ and $D=\left\{z_{1} \cdot z_{2}=\right.$ $0\}$. We put $O=(0,0)$. We have already known that ${ }^{\diamond} \mathcal{E}^{0}$ is locally free over $X-\{O\}$. We can show that we have a sections $f_{1}, \ldots, f_{N}$ of ${ }^{\diamond} \mathcal{E}^{0}$ such that they generate ${ }^{\diamond} \mathcal{E}^{0}$ over $X-\{O\}$. Use, for example, an argument in page 35 in [11]. Then we obtain the morphism $\varphi$ : $\mathcal{O}^{\oplus N} \longrightarrow{ }^{\diamond} \mathcal{E}^{0}$. The image of $\varphi$ is denoted by $\operatorname{Im}(\varphi)$, which is coherent. On $X-\{O\}$, we have $\operatorname{Im}(\varphi)={ }^{\diamond} \mathcal{E}^{0}$. Consider the double dual of $\operatorname{Im}(\varphi)$, namely, we put $\mathcal{F}:=\operatorname{Hom}(\operatorname{Im}(\varphi, \mathcal{O}), \mathcal{O})$. Then $\mathcal{F}$ is coherent, and locally free. Since $\mathcal{F}$ is locally free, we have ${ }^{\diamond} \mathcal{E}^{0} \subset \mathcal{F}$ due to Hartogs theorem. On the other hand, we can show that the increasing orders any holomorphic sections of $\mathcal{F}$ are less than 0, due to Corollary 4.12. Thus we have $\mathcal{F}={ }^{\diamond} \mathcal{E}^{0}$.

The argument partially works in higher dimensional case, i.e., we can show that ${ }^{\diamond} \mathcal{E}^{0}$ is coherent and reflexive by the same argument. But the double dual of coherent sheaf is just reflexive, and not locally free in general.

However, the referee kindly informed to the author that we can derive the local freeness of ${ }^{\diamond} \mathcal{E}^{0}$ by using the argument above, the resolution of the singularity of coherent sheaves, the functoriality below, and some 'semistability' argument of vector bundles. 


\subsection{Functoriality}

Proposition 4.10. We have the following functoriality of the prolongment.

$$
\begin{array}{ll}
\operatorname{det}\left({ }^{\diamond} \mathcal{E}\right) \simeq{ }^{\diamond}(\operatorname{det}(\mathcal{E})), & { }^{\diamond} \mathcal{E}_{1} \otimes \diamond \mathcal{E}_{2} \simeq{ }^{\diamond}\left(\mathcal{E}_{1} \otimes \mathcal{E}_{2}\right), \\
\diamond\left(\mathcal{E}^{\vee}\right) \simeq\left({ }^{\diamond}\right)^{\vee} . & \operatorname{Hom}\left({ }^{\diamond} \mathcal{E}_{1},{ }^{\diamond} \mathcal{E}_{2}\right) \simeq{ }^{\diamond} \operatorname{Hom}\left(\mathcal{E}_{1}, \mathcal{E}_{2}\right) \\
\operatorname{Sym}^{l}\left({ }^{\diamond} \mathcal{E}\right) \simeq{ }^{\diamond} \operatorname{Sym}^{l} \mathcal{E}, & \bigwedge^{l}\left({ }^{\diamond} \mathcal{E}\right) \simeq \bigwedge^{l} \mathcal{E} .
\end{array}
$$

In each case, the $\lambda$-connection $\mathbb{D}$ is also isomorphic.

We also a similar isomorphisms of the conjugate deformed holomorphic bundles with $\mu$-connections.

Proof. Consider the case of determinant bundles. We have the natural morphism $\operatorname{det}\left({ }^{\diamond} \mathcal{E}\right) \longrightarrow{ }^{\diamond} \operatorname{det}(\mathcal{E})$. By using the result of Simpson (see [35], or Lemma 4.4 in this paper) and the extension property of the holomorphic sections, we can show that the morphism is surjective, and thus isomorphic. The other cases are similar. q.e.d.

Consider the morphism $f: \Delta_{z}^{m} \longrightarrow \Delta_{\zeta}^{n}$ given as follows:

$$
f^{*}\left(\zeta_{i}\right)=\prod_{j} z_{j}^{a_{i j}}
$$

Let $\left(E, \bar{\partial}_{E}, \theta, h\right)$ be a tame nilpotent harmonic bundle with trivial parabolic structure over $\Delta_{\zeta}^{* n}$. Then the deformed holomorphic bundle of $f^{*}\left(E, \bar{\partial}_{E}, \theta, h\right)$ is naturally isomorphic to the pull back of $(\mathcal{E}, \mathbb{D}, h)$.

Proposition 4.11. We have the natural isomorphism of $f^{*}\left({ }^{\diamond} \mathcal{E}\right)$ and $^{\diamond}\left(f^{*} \mathcal{E}\right)$.

Proof. Since the prolongment is characterized by increasing orders, we have the natural morphism $f^{*}\left({ }^{\diamond} \mathcal{E}\right) \longrightarrow{ }^{\diamond}\left(f^{*} \mathcal{E}\right)$. We have to show that it is isomorphic. When rank of $E$ is 1 , then the claim is obvious. Consider the general case. Let $\boldsymbol{v}$ be a holomorphic frame of ${ }^{\diamond} \mathcal{E}$. Then $f^{*} \Omega(\boldsymbol{v})$ gives a holomorphic frame of $f^{*} \operatorname{det}\left({ }^{\diamond} \mathcal{E}\right) \simeq \operatorname{det}\left({ }^{\diamond} f^{*} \mathcal{E}\right)$. Thus $f^{*}(\boldsymbol{v})$ naturally gives a frame of ${ }^{\diamond} f^{*} \mathcal{E}$.

q.e.d.

We see the relation of prolongments of $\mathcal{E}$ and $\mathcal{E}^{\dagger}$. We put $X=\Delta^{n}$ and $D=\left\{\prod_{i=1}^{n} z_{i}=0\right\}$. Let $\boldsymbol{v}$ be a holomorphic frame of ${ }^{\diamond} \mathcal{E}$ over $\mathcal{X}$. We have the restriction $\boldsymbol{v}_{\mid \mathcal{X}-\mathcal{D}}$ of $\boldsymbol{v}$ to $\mathcal{X}-\mathcal{D}$, which is a frame of $\mathcal{E}$ over $\mathcal{X}-\mathcal{D}$. Then we obtain the conjugate frame $\left(\boldsymbol{v}_{\mid \mathcal{X}-\mathcal{D}}\right)^{\dagger}$ of $\mathcal{E}^{\dagger}$ over $\mathcal{X}^{\dagger}-\mathcal{D}^{\dagger}$ 
Proposition 4.12. $\left(\boldsymbol{v}_{\mid \mathcal{X}-\mathcal{D}}\right)$ naturally induces the frame of ${ }^{\diamond} \mathcal{E}^{\dagger}$ over $\mathcal{X}^{\dagger}$.

Proof. When the rank of $E$ is 1, the claim is obvious. Consider the general case. By using the result in the case of curves, we can show that the increasing order of $v_{i \mid \mathcal{X}-\mathcal{D}}^{\dagger}$ is less than 0 , and thus it gives a holomorphic section of ${ }^{\diamond} \mathcal{E}^{\dagger}$. Consider the section $\Omega\left(\boldsymbol{v}_{\mathcal{X}-\mathcal{D}}^{\dagger}\right)$. By

functoriality of our construction, it is same as $\Omega(\boldsymbol{v})_{\mathcal{X}-\mathcal{D}}^{\dagger}$, which gives a holomorphic frame of $\operatorname{det}\left({ }^{\diamond} \mathcal{E}^{\dagger}\right)$. Thus $\boldsymbol{v}_{\mathcal{X}-\mathcal{D}}^{\dagger}$ gives a frame of ${ }^{\diamond} \mathcal{E}^{\dagger}$. q.e.d.

We will denote the induced frame by $\boldsymbol{v}_{\mid \mathcal{X}-\mathcal{D}}^{\dagger}$ simply by $\boldsymbol{v}^{\dagger}$.

\section{Limiting mixed twistor structure}

\subsection{Preliminary}

\subsubsection{Normalizing frame}

Let $\left(E, \mathbb{D}^{1}\right)$ be a holomorphic vector bundle with a holomorphic flat connection over $\Delta^{* n}$. Assume that the monodromies are unipotent. Fix the coordinate $\left(z_{1}, \ldots, z_{n}\right)$ of $\Delta^{* n}$. Recall that we have a holomorphic frame such that the corresponding connection form is of the form $\sum_{i} A_{i} d z_{i} / z_{i}$ for $A_{i} \in M(r)$. Such a frame is called the normalizing frame in this paper. Let us recall the construction of such a frame.

Let $\mathbb{H}$ denote the upper half plain $\{\zeta \in \mathbf{C} \mid \operatorname{Im}(\zeta)>0\}$. Take the universal covering $\pi: \mathbb{H}^{n} \longrightarrow \Delta^{* n}$, given by $z_{i}=\exp \left(2 \pi \sqrt{-1} \zeta_{i}\right)$. Let $P$ be a point of $\Delta^{* n}$ and $\widetilde{P}$ be a point of $\mathbb{H}^{n}$ such that $\pi(\widetilde{P})=P$. Let $\gamma_{i}$ denote the following path:

$$
\begin{aligned}
\{t \in \mathbf{R} \mid 0 \leq t \leq 1\} \ni t \\
\\
\longmapsto\left(z_{1}, \ldots, z_{i-1}, \exp (2 \pi \sqrt{-1} t) \cdot z_{i}, z_{i+1}, \ldots, z_{n}\right) \in \Delta^{* n} .
\end{aligned}
$$

We denote the monodromy along $\gamma_{i}$ by $M\left(\gamma_{i}\right)$.

Pick a base $\boldsymbol{e}(P)$ of $E_{\mid P}$. Let $M_{i}$ be the matrix representing the endomorphism $M\left(\gamma_{i}\right) \in \operatorname{End}\left(E_{\mid P}\right)$ with respect to the frame $\boldsymbol{w}(P)$, that is, $M\left(\gamma_{i}\right) \boldsymbol{w}=\boldsymbol{e} \cdot M_{i}$. By our assumption, $M_{i}$ is unipotent. We denote the logarithm of $M_{i}$ by $N_{i}$, i.e.,

$$
N_{i}=\log \left(M_{i}\right):=\sum_{m=l}^{\infty} \frac{1}{m}\left(M_{i}-1\right)^{m}, \quad \exp \left(N_{i}\right)=M_{i} .
$$


The frame $e(P)$ of $E_{\mid P}$ naturally induces the base $\boldsymbol{e}(\widetilde{P})$ of $\pi^{*} E_{\mid \widetilde{P}}$. We can take the flat frame $\boldsymbol{u}$ of $\pi^{*} E$ such that $\boldsymbol{u}_{\mid \widetilde{P}}=\boldsymbol{e}(\widetilde{P})$. Then we put as follows:

$$
\boldsymbol{w}=\boldsymbol{u} \cdot \exp \left(-\sum_{i=1}^{n}\left(\zeta_{i}-\zeta_{i}(\widetilde{P})\right) \cdot N_{i}\right)
$$

Then $\boldsymbol{w}$ is, in fact, the pull back of some holomorphic frame of $E$ on $\Delta^{* n}$. We denote the frame over $\Delta^{* n}$ by the same notation $\boldsymbol{w}$. We have the following equality:

$$
\mathbb{D}^{1} \boldsymbol{w}=\boldsymbol{v} \cdot\left(-\sum_{i=1}^{n} N_{i} \cdot d \zeta_{i}\right)=\boldsymbol{w} \cdot\left(-\sum_{i=1}^{n} N_{i} \cdot \frac{d z_{i}}{2 \pi \sqrt{-1} z_{i}}\right)
$$

Note that such normalizing frame is determined once we pick a point $P$ and the frame $\boldsymbol{e}(P)$, when we fix a coordinate $\left(z_{1}, \ldots, z_{n}\right)$. Namely, we have the following lemma.

Lemma 5.1. Let $\boldsymbol{w}_{1}$ and $\boldsymbol{w}_{2}$ be normalizing frames such that $\boldsymbol{w}_{1}(P)$ $=\boldsymbol{w}_{2}(P)$. Then we have $\boldsymbol{w}_{1}=\boldsymbol{w}_{2}$.

Proof. On $\mathbb{H}^{n}$, the frames $\boldsymbol{w}_{i}$ are solutions of Equation (46), such that $\boldsymbol{w}_{1}(\widetilde{P})=\boldsymbol{w}_{2}(\widetilde{P})$. Such solution is uniquely determined. q.e.d.

Let us see the dependence of a normalizing frame $\boldsymbol{w}$ on a choice of $P, \boldsymbol{e}(P)$ and the coordinate. Take another frame $\boldsymbol{e}_{1}(P)$ of $E_{\mid P}$. We denote the corresponding normalizing frame by $\boldsymbol{w}_{1}$. We have an element $g \in G L(r)$ such that $\boldsymbol{e}_{1}(P)=\boldsymbol{e}(P) \cdot g$. Then we have $\boldsymbol{w}_{1}(P)=\boldsymbol{w}(P) \cdot g$. We also have $\mathbb{D}^{1} \boldsymbol{w} \cdot g=\boldsymbol{w} \cdot A \cdot g=\boldsymbol{w} \cdot g \cdot\left(g^{-1} \cdot A \cdot g\right)$. Thus we obtain that $\boldsymbol{w}_{1}=\boldsymbol{w} \cdot g$.

Let $Q$ be another point of $\Delta^{* n}$, and $\boldsymbol{e}(Q)$ be a base of $E_{\mid Q}$. We denote the corresponding normalizing frame by $\boldsymbol{w}_{2}$. We have an element $g \in G L(r)$ such that $\boldsymbol{w}_{2}(P)=\boldsymbol{w}(P) \cdot g$. Then we know that $\boldsymbol{w}_{2}=\boldsymbol{w} \cdot g$.

Let $\left(z_{1}^{\prime}, \ldots, z_{n}^{\prime}\right)$ denote another holomorphic coordinate system, satisfying $\left\{z_{i}^{\prime}=0\right\}=\left\{z_{i}=0\right\}=: D_{i}$ for any $i$. Note that $\left(z_{i}^{\prime}\right)$ be a coordinate of an open subset $U$ of $\mathbf{C}^{n}$, which is not necessarily same as $\Delta^{n}$ above. Take a covering $\pi^{\prime}: \mathbb{H}^{n} \longrightarrow U-\bigcup_{i} D_{i}$. We use the coordinate $\left(\zeta_{i}^{\prime}\right)$ for $\mathbb{H}^{n}$ in this case. We denote the corresponding normalizing frame $\boldsymbol{w}^{\prime}$.

We have a holomorphic function $g_{i}$ defined over $\Delta^{n}$ satisfying $\left(\zeta_{i}-\right.$ $\left.\zeta_{i}^{\prime}\right)=g_{i}$, or $z_{i} / z_{i}^{\prime}=\exp \left(2 \pi \sqrt{-1} g_{i}\right)$. Then we have the following relation: 


$$
\begin{array}{r}
\boldsymbol{w}^{\prime}=\boldsymbol{u} \cdot \exp \left(-\sum\left(\zeta_{i}^{\prime}-\zeta_{i}^{\prime}(P)\right) \cdot N_{i}\right) \\
=\boldsymbol{u} \cdot \exp \left(-\sum\left(\zeta_{i}-\zeta_{i}(P)\right) \cdot N_{i}+\sum_{i}\left(g_{i}+\left(\zeta_{i}^{\prime}(P)-\zeta_{i}(P)\right)\right) \cdot N_{i}\right) \\
=\boldsymbol{w} \cdot \exp \left[\sum_{i}\left(g_{i}+\left(\zeta_{i}^{\prime}(P)-\zeta_{i}(P)\right)\right) \cdot N_{i}\right] .
\end{array}
$$

\subsubsection{Isomorphisms}

Let $\boldsymbol{w}=\left(w_{1}, \ldots, w_{r}\right)$ be a normalizing frame of a holomorphic flat connection $\left(E, \mathbb{D}^{1}\right)$. We regard it as a frame of the prolongment ${ }^{\diamond} E$ over $\Delta^{n}$. Let $P$ and $Q$ be points of $\Delta^{n}$. Let $v$ be an element of ${ }^{\diamond} E_{\mid P}$. Then $v$ can be uniquely described as a linear combination of $w_{i \mid P}$, that is, $v=\sum_{i} v_{i} \cdot w_{i \mid P}$ for $v_{i} \in \mathbf{C}$. Then we obtain the element $\Phi_{P, Q}(v)=\sum_{i} v_{i} \cdot w_{i \mid Q}$ of ${ }^{\diamond} E_{\mid Q}$. Then we obtain the linear isomorphism $\Phi_{(P, Q)}:{ }^{\diamond} E_{\mid P} \longrightarrow{ }^{\diamond} E_{\mid Q}$.

Lemma 5.2. When we fix a coordinate of $\Delta^{n}$, then the morphism $\Phi_{(P, Q)}$ is independent of a choice of normalizing frame.

Proof. Let $\boldsymbol{w}_{1}$ and $\boldsymbol{w}_{2}$ be normalizing frames. Then we have the element $g \in G L(r, \mathbf{C})$ such that $\boldsymbol{w}_{1}=\boldsymbol{w}_{2} \cdot g$. Thus we have the relation $\boldsymbol{w}_{1 \mid P}=\boldsymbol{w}_{2 \mid P} \cdot g$ and $\boldsymbol{w}_{1 \mid Q}=\boldsymbol{w}_{2 \mid Q} \cdot g$. It implies that $\Phi_{(P, Q)}$ is independent of a choice of a normalizing frame. $\quad$ q.e.d.

Let $P$ be a point contained in $\Delta^{* n}$. Then we have the action of monodromies $M\left(\gamma_{i}\right)$ and the logarithms $N\left(\gamma_{i}\right)$ on $E_{\mid P}$. Let $P$ be a point contained in $D_{i}=\left\{z_{i}=0\right\}$. Then we have the endomorphism $\operatorname{Res}_{D_{i}}\left(\mathbb{D}^{1}\right)_{\mid P}$ of ${ }^{\diamond} E_{\mid P}$. We denote it by $N\left(\gamma_{i}\right)$ by abbreviation.

Lemma 5.3. Let $P$ and $Q$ be points of $\Delta^{n}$. Then the isomorphism $\Phi_{(P, Q)}$ is compatible with $N\left(\gamma_{i}\right)$.

Proof. Both of the endomorphisms $N\left(\gamma_{i}\right)_{\mid P}$ and $N\left(\gamma_{i}\right)_{\mid Q}$ are represented by $N_{i}$ with respect to the frames $\boldsymbol{w}_{\mid P}$ and $\boldsymbol{w}_{\mid Q}$. Thus the isomorphism $\Phi_{(P, Q)}$ are compatible with $N\left(\gamma_{i}\right)$ q.e.d.

We see the dependence of $\Phi_{(P, Q)}$ on a choice of the coordinate. Let $\left(z_{i}^{\prime}\right)$ be another coordinate. We denote the corresponding morphism by $\Phi_{(P, Q)}^{\prime}$. 
Lemma 5.4. We have the relation

$$
\Phi_{(P, Q)}=\exp \left(\sum_{i}\left(g_{i}(P)-g_{i}(Q)\right) \cdot N\left(\gamma_{i}\right)\right) \circ \Phi_{(P, Q)}^{\prime} .
$$

Proof. We have the relation (47) between $\boldsymbol{w}$ and $\boldsymbol{w}^{\prime}$. It implies the relation of $\Phi_{(P, Q)}$ and $\Phi_{(P, Q)}^{\prime}$.

q.e.d.

\subsection{The vector bundle of Simpson}

\subsubsection{Normalizing frames}

We put $X=\Delta^{n}, D_{i}=\left\{z_{i}=0\right\}$ and $D=\bigcup_{i=1}^{l} D_{i}$ for $l \leq n$. Let $\left(E, \bar{\partial}_{E}, \theta, h\right)$ be a tame nilpotent harmonic bundle with trivial parabolic structure over $X-D$. We have the prolongment of the deformed holomorphic bundle ${ }^{\diamond} \mathcal{E}$ over $\mathcal{X}$, and the $\lambda$-connection $\mathbb{D}^{\lambda}$ of log type. The following lemma is easy from the construction of normalizing frames.

Lemma 5.5. We can take a holomorphic frame $\boldsymbol{w}$ of $\mathcal{E}^{\sharp}$ over $\mathcal{X}^{\sharp}$ such that the restrictions $\boldsymbol{w}_{\mid \mathcal{X}^{\lambda}}$ are normalizing frames for $\mathbb{D}^{\lambda, f}$.

In that case, the following holds: Let $\mathcal{A}^{f} \in \Gamma\left(\mathcal{X}^{\sharp}, M(r) \otimes p_{\lambda}^{*} \Omega_{X}^{1,0}\right)$ be a holomorphic flat connection form of $\mathbb{D}^{f}$ with respect to $\boldsymbol{w}$, that is, $\mathbb{D}^{f} \cdot \boldsymbol{w}=\boldsymbol{w} \cdot \mathcal{A}^{f}$ over $\mathcal{X}^{\sharp}$. Then $\mathcal{A}^{f}$ is of the following form:

$$
\sum_{i} C_{i}(\lambda) \cdot \frac{d z_{i}}{z_{i}}
$$

Here $C_{i}(\lambda)$ are $M(r)$-valued holomorphic functions of $\lambda$, but they are independent of $z_{i}$. Consider the $\lambda$-connection form $\mathcal{A}$ of $\mathbb{D}$ with respect to the frame $\boldsymbol{w}$. Then we have the relation $\mathcal{A}^{f}=\lambda^{-1} \cdot \mathcal{A}$. Thus $\mathcal{A}$ is also of the form as in (48). A frame such as $\boldsymbol{w}$ above is also called a normalizing frame for $\mathbb{D}$.

Note that there does not exist a normalizing frame for Higgs field. Namely the normalizing frame is defined only over $\mathcal{X}^{\sharp}$, in general.

\subsubsection{Construction}

Let $P$ and $Q$ be points of $X$. As in Subsubsection 5.1.2, we obtain the following isomorphisms by using the normalizing frame for any $\lambda \neq 0$ :

$$
\Phi_{\lambda, P, Q}:{ }^{\diamond} \mathcal{E}_{\mid(\lambda, P)} \longrightarrow{ }^{\diamond} \mathcal{E}_{\mid(\lambda, Q)}
$$


Since $\boldsymbol{w}$ is holomorphic with respect to $\lambda$, the dependence of the morphisms $\Phi_{\lambda, P, Q}$ on $\lambda$ is holomorphic. Thus we obtain the holomorphic isomorphism of the following locally free sheaves over $\mathbf{C}_{\lambda}^{*}$ for any $P, Q \in X$ :

$$
\Phi_{\lambda, P, Q}:{ }^{\diamond} \mathcal{E}^{\sharp}\left|\mathbf{C}_{\lambda}^{*} \times\{P\} \longrightarrow{ }^{\diamond} \mathcal{E}^{\sharp}\right| \mathbf{C}_{\lambda}^{*} \times\{P\} .
$$

Similarly we obtain the holomorphic isomorphism for any $P, Q \in X^{\dagger}$ :

$$
\Phi_{\mu, P, Q}^{\dagger}:{ }^{\diamond} \mathcal{E}^{\dagger \sharp} \mid \mathbf{C}_{\mu}^{*} \times\{P\} \longrightarrow{ }^{\diamond} \mathcal{E}^{\dagger}{ }_{\mid \mathbf{C}_{\mu}^{*} \times\{P\}} .
$$

We have the isomorphism $\mathbf{C}_{\lambda}^{*} \simeq \mathbf{C}_{\mu}^{*}$ given by $\mu=\lambda^{-1}$. Recall that we have the following identification of $C^{\infty}$-bundles with the families of the flat connections (Lemma 3.5.):

$$
\left(\mathcal{E}^{\sharp}, \mathbb{D}^{f}\right)=\left(\mathcal{E}^{\dagger \sharp}, \mathbb{D}^{\dagger f}\right) \text {, over } \mathcal{X}^{\sharp}-\mathcal{D}^{\sharp}=\mathcal{X}^{\dagger \sharp}-\mathcal{D}^{\dagger \sharp} \text {. }
$$

Note that they are identification of the $C^{\infty}$-vector bundles, and the identification is holomorphic with respect to $\lambda$ and $\mu$. Let $P$ be a point of $X-D$. Then we have the following identification of holomorphic bundles over $\mathbf{C}_{\lambda}^{*}=\mathbf{C}_{\mu}^{*}$ :

$$
\mathcal{E}_{\mid \mathbf{C}_{\lambda}^{*} \times\{P\}}^{\sharp}=\mathcal{E}_{\mid \mathbf{C}_{\mu}^{*} \times\{P\}}^{\sharp} \cdot
$$

Let $Q_{1}$ and $Q_{2}$ be points of $X$ and let $P$ be a point of $X-D$. Then we obtain the following sequence of isomorphisms:

$$
{ }^{\diamond} \mathcal{E}^{\sharp} \mid \mathbf{C}_{\lambda}^{*} \times\left\{Q_{1}\right\} \stackrel{\Phi_{\lambda, Q_{1}, P}}{\longrightarrow} \mathcal{E}_{\mid \mathbf{C}_{\lambda}^{*} \times\{P\}}^{\sharp}=\mathcal{E}_{\mid \mathbf{C}_{\mu}^{*} \times\{P\}}^{\dagger} \stackrel{\Phi_{\mu, P, Q_{2}}^{\dagger}}{\longrightarrow} \mathcal{E}^{\dagger \sharp} \mathbf{C}_{\mu}^{*} \times\left\{Q_{2}\right\} .
$$

We denote the composite by $\Psi\left(Q_{1}, Q_{2}, P\right)$. We have the vector bundles $\mathcal{E}_{\mid \mathbf{C}_{\lambda} \times\left\{Q_{1}\right\}}$ over $\mathbf{C}_{\lambda}$ and $\mathcal{E}_{\mid \mathbf{C}_{\mu} \times\left\{Q_{2}\right\}}^{\dagger}$ over $\mathbf{C}_{\mu}$. By the gluing $\Psi\left(Q_{1}, Q_{2}, P\right)$, we obtain the holomorphic vector bundle over $\mathbb{P}^{1}=\mathbf{C}_{\lambda} \cup \mathbf{C}_{\mu}$. The vector bundle is denoted by $S\left(Q_{1}, Q_{2}, P,\left(z_{i}\right)\right)$. When we fix the coordinate, then we often omit to denote $\left(z_{i}\right)$. When $Q_{1}=Q_{2}=Q$, it is denoted by $S(Q, P)$. When we distinguish the harmonic bundle $\left(E, \bar{\partial}_{E}, \theta, h\right)$, it is denoted by $S\left(Q, P,\left(E, \bar{\partial}_{E}, \theta, h\right)\right)$. The vector bundle $S\left(Q_{1}, Q_{2}, P\right)$ is independent of a choice of normalizing frame, when we fix a coordinate, due to Lemma 5.2. 


\subsubsection{Nilpotent maps}

Assume that $Q_{1}$ and $Q_{2}$ are contained in $D_{i}$. Then we have the following morphisms of coherent sheaves:

$$
\operatorname{Res}_{\mathcal{D}_{i}}(\mathbb{D}) \in \operatorname{End}\left({ }^{\diamond} \mathcal{E}_{\mid \mathbf{C}_{\lambda} \times\left\{Q_{1}\right\}}\right), \quad \operatorname{Res}_{\mathcal{D}_{i}^{\dagger}}\left(\mathbb{D}^{\dagger}\right) \in \operatorname{End}\left({ }^{\diamond} \mathcal{E}^{\dagger} \mid \mathbf{C}_{\mu} \times\left\{Q_{2}\right\}\right) .
$$

Lemma 5.6. The nilpotent morphisms $\operatorname{Res}_{\mathcal{D}_{i}}(\mathbb{D})$ and $-\operatorname{Res}_{\mathcal{D}_{i}^{\dagger}}\left(\mathbb{D}^{\dagger}\right)$ induce the morphism of the coherent sheaves:

$$
\mathcal{N}_{i}^{\triangle}: S\left(Q_{1}, Q_{2}, P\right) \longrightarrow S\left(Q_{1}, Q_{2}, P\right) \otimes \mathcal{O}_{\mathbb{P}^{1}}(2) .
$$

Proof. Then we have the following morphisms of the coherent sheaves:

$$
\operatorname{Res}_{\mathcal{D}_{i}}\left(\mathbb{D}^{f}\right) \in \operatorname{End}\left({ }^{\diamond} \mathcal{E}_{\mid \mathbf{C}_{\lambda} \times\left\{Q_{1}\right\}}\right), \quad \operatorname{Res}_{\mathcal{D}_{i}^{\dagger}}\left(\mathbb{D}^{\dagger f}\right) \in \operatorname{End}\left({ }^{\diamond} \mathcal{E}^{\dagger} \mid \mathbf{C}_{\mu} \times\left\{Q_{2}\right\}\right) .
$$

The relation of $\operatorname{Res}_{\mathcal{D}_{i}}\left(\mathbb{D}^{f}\right)$ and $\operatorname{Res}_{\mathcal{D}_{i}}(\mathbb{D})$, (resp. $\operatorname{Res}_{\mathcal{D}_{i}^{\dagger}}\left(\mathbb{D}^{\dagger f}\right)$ and $\left.\operatorname{Res}_{\mathcal{D}_{i}^{\dagger}}\left(\mathbb{D}^{\dagger}\right)\right)$ is given as follows:

$$
\operatorname{Res}_{\mathcal{D}_{i}}\left(\mathbb{D}^{f}\right)=\lambda^{-1} \cdot \operatorname{Res}_{\mathcal{D}_{i}}(\mathbb{D}), \quad\left(\text { resp. } \operatorname{Res}_{\mathcal{D}_{i}^{\dagger}}\left(\mathbb{D}^{\dagger f}\right)=\mu^{-1} \cdot \operatorname{Res}_{\mathcal{D}_{i}^{\dagger}}\left(\mathbb{D}^{\dagger}\right) .\right)
$$

On the other hand, the logarithms of the monodromies of the flat connections $\mathbb{D}$ along the path $\gamma_{i}$, induces the following morphism of the coherent sheaves:

$$
N\left(\gamma_{i}\right) \in \operatorname{End}\left({ }^{\diamond} \mathcal{E}_{\mid \mathbf{C}_{\lambda} \times\{P\}}\right) .
$$

By the morphisms $\Phi_{\lambda, Q_{1}, P}$, the $\operatorname{Res}_{\mathcal{D}_{i}}\left(\mathbb{D}^{f}\right)$ is mapped to $N\left(\gamma_{i}\right)$. On the other hand, the morphism $\Phi_{\mu, Q_{2}, P}^{\dagger}$ map the $\operatorname{Res}_{\mathcal{D}_{i}^{\dagger}}\left(\mathbb{D}^{\dagger f}\right)$ to $-N\left(\gamma_{i}\right)$. Thus we are done. q.e.d.

\subsubsection{Gluing matrices}

Let $\boldsymbol{v}$ be a holomorphic frame of $\diamond \mathcal{E}$ over $\mathcal{X}$. Then $\boldsymbol{v}^{\dagger}$ is naturally a holomorphic frame of ${ }^{\diamond} \mathcal{E}^{\dagger}$ over $\mathcal{X}^{\dagger}$. We obtain the frames $\boldsymbol{v}_{\mid \mathbf{C}_{\lambda} \times Q_{1}}$ and $\boldsymbol{v}_{\mid \mathbf{C}_{\mu} \times Q_{2}}^{\dagger}$ of ${ }^{\diamond} \mathcal{E}_{\mid \mathbf{C}_{\lambda} \times Q_{1}}$ and ${ }^{\diamond} \mathcal{E}^{\dagger} \mathbf{C}_{\mu} \times Q_{2}$ respectively. We would like to describe the gluing morphism $\Psi\left(Q_{1}, Q_{2}, P\right)$ with respect to the frames.

Let $\boldsymbol{w}$ be a normalizing frame of $\mathcal{E}^{\sharp}$ for the flat connections $\mathbb{D}^{f}$. Then $\boldsymbol{w}^{\dagger}$ is naturally a normalizing frame of $\mathcal{E}^{\dagger \sharp}$ for the flat connection $\mathbb{D}^{\dagger f}$. Let $\Gamma \in C^{\infty}\left(\mathcal{X}^{\sharp}, G L(r)\right)$ be the transformation matrices of $\boldsymbol{v}$ and $\boldsymbol{w}$ that is, $\boldsymbol{w}=\boldsymbol{v} \cdot \Gamma$. Then the transformation matrices of $\boldsymbol{v}^{\dagger}$ and $\boldsymbol{w}^{\dagger}$ is given 
by $\Gamma^{\dagger}=F^{*}\left({ }^{t} \bar{\Gamma}^{-1}\right)$, that is, $\boldsymbol{w}^{\dagger}=\boldsymbol{v}^{\dagger} \cdot \Gamma^{\dagger}$. Here $F$ denotes the morphism $\mathbf{C}_{\mu} \longrightarrow \mathbf{C}_{\lambda}^{\dagger}$ given by $\mu=-\bar{\lambda}$ (see Subsubsection 3.1.6).

Let $\boldsymbol{e}$ be a frame of $\mathcal{E}^{0}$ over $\mathcal{X}^{0}-\mathcal{D}^{0}$. Then $\boldsymbol{e}^{\dagger}$ gives a frame of $\mathcal{E}^{\dagger 0}$. The relation of $\boldsymbol{e}$ and $\boldsymbol{e}^{\dagger}$ is given by $\boldsymbol{e}^{\dagger}=\boldsymbol{e} \cdot \overline{H(h, e)}^{-1}$.

Let $B \in C^{\infty}(\mathcal{X}-\mathcal{D}, G L(r))$ be a transformation matrices of $p_{\lambda}^{-1}(\boldsymbol{e})$ and $\boldsymbol{v}$, that is, $\boldsymbol{v}=p_{\lambda}^{-1}(\boldsymbol{e}) \cdot B$. Then the transformation matrices of $\boldsymbol{v}^{\dagger}$ and $p_{\mu}^{\dagger-1}\left(\boldsymbol{e}^{\dagger}\right)$ is given by $B^{\dagger}=F^{*}\left({ }^{t} \bar{B}^{-1}\right)$, that is, $\boldsymbol{v}^{\dagger}=p_{\mu}^{\dagger-1}\left(\boldsymbol{e}^{\dagger}\right) \cdot B^{\dagger}$.

Then the gluing morphism $\Psi\left(Q_{1}, Q_{2}, P\right)$ for $S\left(Q_{1}, Q_{2}, P\right)$ is represented by the following $G L(r)$-valued function $A(\lambda, P)$, with respect to the frames $\boldsymbol{v}_{\mid \mathbf{C}_{\lambda}^{*} \times Q_{1}}$ and $\boldsymbol{v}_{\mid \mathbf{C}_{\mu}^{*} \times Q_{2}}^{\dagger}$ :

$$
\begin{aligned}
\boldsymbol{v}_{\mid \mathbf{C}_{\mu}^{*} \times Q_{2}}^{\dagger} & =\boldsymbol{v}_{\mid \mathbf{C}_{\lambda}^{*} \times Q_{1}} \cdot A(\lambda, P) \\
A(\lambda, P):= & \Gamma\left(\lambda, Q_{1}\right) \cdot \Gamma(\lambda, P)^{-1} \cdot B(\lambda, P)^{-1} \\
& \cdot \overline{H(h, e)}^{-1}(P) \cdot B^{\dagger}(\mu, P) \cdot \Gamma^{\dagger}(\mu, P) \cdot \Gamma^{\dagger}\left(\mu, Q_{2}\right)^{-1} .
\end{aligned}
$$

Here $\mu=\lambda^{-1}$.

\subsubsection{Functoriality}

We show the functoriality of the construction of the vector bundle $S(O, P)$. To distinguish the harmonic bundle $\left(E, \bar{\partial}_{E}, \theta, h\right)$, we use the notation $S\left(O, P,\left(E, \bar{\partial}_{E}, \theta, h\right)\right)$.

Proposition 5.1. We fix the coordinate of $\Delta^{n}$. Then we have the following natural isomorphisms:

$$
\begin{aligned}
& \operatorname{det}\left(S\left(O, P,\left(E, \bar{\partial}_{E}, \theta, h\right)\right)\right) \simeq S\left(O, P, \operatorname{det}\left(E, \bar{\partial}_{E}, \theta, h\right)\right), \\
& S\left(O, P,\left(E_{1}, \theta_{1}, h_{1}\right)\right) \otimes S\left(O, P,\left(E_{2}, \theta_{2}, h_{2}\right)\right) \\
& \simeq S\left(O, P,\left(E_{1}, \theta_{1}, h_{1}\right) \otimes\left(E_{2}, \theta_{2}, h_{2}\right)\right), \\
& S\left(O, P,\left(E, \bar{\partial}_{E}, \theta, h\right)\right)^{\vee} \simeq S\left(O, P,\left(E^{\vee}, \bar{\partial}_{E^{\vee}}, \theta^{\vee}, h^{\vee}\right)\right), \\
& \operatorname{Hom}\left(S\left(O, P,\left(E_{1}, \theta_{1}, h_{1}\right)\right), S\left(O, P,\left(E_{2}, \theta_{2}, h_{2}\right)\right)\right) \\
& \simeq S\left(O, P, \operatorname{Hom}\left(\left(E_{1}, \theta_{1}, h_{1}\right),\left(E_{2}, \theta_{2}, h_{2}\right)\right)\right), \\
& S\left(O, P, \operatorname{Sym}^{l}\left(E, \bar{\partial}_{E}, \theta, h\right)\right) \simeq \operatorname{Sym}^{l} S\left(O, P,\left(E, \bar{\partial}_{E}, \theta, h\right)\right), \\
& \quad l \\
& S\left(O, P, \bigwedge\left(E, \bar{\partial}_{E}, \theta, h\right)\right) \simeq \bigwedge S\left(O, P,\left(E, \bar{\partial}_{E}, \theta, h\right)\right),
\end{aligned}
$$


In each isomorphism, the nilpotent morphisms $N_{i}^{\triangle}$ are also isomorphic, if $O$ is contained in $D_{i}=\left\{z_{i}=0\right\}$.

Proof. Consider the case of the tensor product. Let $\boldsymbol{w}_{a}=\left(w_{a i}\right)$ be normalizing frames of $\diamond \mathcal{E}_{a}$ over $\mathbf{C}_{\lambda} \times \Delta^{n}$ for $a=1,2$. Then $\boldsymbol{w}_{1} \otimes \boldsymbol{w}_{2}=$ $\left(w_{1 i} \otimes w_{2 j}\right)$ gives a normalizing frame of ${ }^{\diamond}\left(\mathcal{E}_{1} \otimes \mathcal{E}_{2}\right)$. Then it is easy to check that our construction is functorial with respect to the tensor product. The other cases are similar.

q.e.d.

Consider the morphism $f: \Delta_{z}^{m} \longrightarrow \Delta_{\zeta}^{n}$ considered in Subsection 4.8, given as follows:

$$
f^{*}\left(\zeta_{i}\right)=\prod_{j} z_{j}^{a_{i j}}
$$

Let $\left(E, \bar{\partial}_{E}, \theta, h\right)$ be a tame nilpotent harmonic bundle with trivial parabolic structure over $\Delta_{\zeta}^{* l} \times \Delta_{\zeta}^{n-l}$.

Proposition 5.2. We have the natural isomorphism of

$$
S\left(Q_{1}, Q_{2}, P, f^{*}\left(E, \bar{\partial}_{E}, \theta, h\right)\right) \simeq S\left(f\left(Q_{1}\right), f\left(Q_{2}\right), f(P),\left(E, \bar{\partial}_{E}, \theta, h\right)\right) .
$$

Proof. Let $\boldsymbol{w}$ be a normalizing frame of ${ }^{\diamond} \mathcal{E}^{\sharp}$. Then $f^{*}(\boldsymbol{w})$ is a normalizing frame of $f^{* \diamond} \mathcal{E}^{\sharp}$. Then the proposition follows from the functoriality of the prolongation (Proposition 4.11).

q.e.d.

\subsection{A limiting mixed twistor theorem}

\subsubsection{The filtration}

Consider the case that the base manifold is one dimensional, i.e., $X=\Delta$ and $D=\{O\}$. Due to the result of Simpson, the conjugacy classes of $\mathcal{N}^{\triangle}(\lambda, O)=\operatorname{Res}\left(\mathbb{D}^{\lambda}\right)$ is independent of a choice of $\lambda$. Thus the weight filtrations $W\left(\operatorname{Res}\left(\mathbb{D}^{\lambda}\right)\right)$ induced by $\operatorname{Res}\left(\mathbb{D}^{\lambda}\right)$ form the filtrations

of $S(O, P)$ by subvector bundles. We denote the filtration by $W^{\triangle}$. Thus we obtain the filtered vector bundle $\left(S(O, P), W^{\triangle}\right)$.

Ideally, we hope that the filtered vector bundle is mixed twistor, namely, we hope that the $l$-th graded part $\mathcal{G} r_{l}$ is a direct sum of $\mathcal{O}_{\mathbb{P}^{1}}(l)$.

We call it a conjecture of Simpson. 


\subsubsection{Example (the case of $\operatorname{Mod}(2, a, C))$}

As an example, let us see what kind of filtered vector bundle is obtained in the case $\operatorname{Mod}(2, a, C)$. (See Subsubsection 3.2.4.) This is an extremely easy example. We have the canonical frame $\boldsymbol{v}=\left(v^{1,0}, v^{0,1}\right)$ of $\mathcal{E}$ over $\mathbf{C}_{\lambda} \times \Delta$. The restriction $\boldsymbol{v}_{\mid \mathbf{C}_{\lambda} \times O}$ gives a frame of $\mathcal{M o d}(2, a, C)_{\mid \mathbf{C}_{\lambda} \times O}$. For simplicity, we omit to denote the notation $\mid \mathbf{C}_{\lambda} \times O$. The weight filtration $W^{\triangle}$ on $\mathbf{C}_{\lambda}$ is obviously given as follows:

$$
W_{-1}^{\triangle}=\left\langle v^{0,1}\right\rangle, \quad W_{1}^{\triangle}=\left\langle v^{1,0}, v^{0,1}\right\rangle .
$$

On the other hand, $\boldsymbol{v}^{\dagger}=\left(v^{\dagger 1,0}, v^{\dagger 0,1}\right)$ gives a holomorphic frame of $\mathcal{M o d}(2, a, C)^{\dagger}$. Then the filtration $W^{\triangle}$ on $\mathbf{C}_{\mu}$ is as follows:

$$
W_{-1}^{\triangle}=\left\langle v^{\dagger 1,0}\right\rangle, \quad W_{1}^{\triangle}=\left\langle v^{\dagger 1,0}, v^{\dagger 0,1}\right\rangle
$$

Let us consider the gluing matrices. Luckily $\boldsymbol{v}$ and $\boldsymbol{v}^{\dagger}$ are normalizing frames. We have the relations (10) between $\boldsymbol{v}$ and $p_{\lambda}^{-1} \boldsymbol{v}_{\mid \mathcal{X}^{0}-\mathcal{D}_{0}}=$ $p_{\lambda}^{-1} \boldsymbol{e}$, and the relation (11) between $\boldsymbol{v}^{\dagger}$ and $p_{\mu}^{\dagger-1} \boldsymbol{v}_{\mid \mathcal{X}^{\dagger 0}-\mathcal{D}^{\dagger 0}}=p_{\mu}^{\dagger-1} \boldsymbol{e}^{\dagger}$. Then the gluing matrices $A(\lambda, P)$ is given as follows:

$$
\begin{aligned}
& \left(\begin{array}{cc}
1 & \lambda \cdot(y+c)^{-1} \\
0 & 1
\end{array}\right) \cdot\left(\begin{array}{cc}
(y+c)^{-1} & 0 \\
0 & y+c
\end{array}\right) \cdot\left(\begin{array}{cc}
1 & 0 \\
-\mu \cdot(y+c)^{-1} & 1
\end{array}\right) \\
& =\left(\begin{array}{cc}
0 & \lambda \\
-\mu & y+c
\end{array}\right) .
\end{aligned}
$$

Here $y$ denotes $-\log |z(P)|$, and we used the relation $\lambda \cdot \mu=1$. Namely, we have the following relation:

$$
\begin{aligned}
\left(v^{\dagger 1,0}, v^{\dagger 0,1}\right) & =\left(v^{1,0}, v^{0,1}\right) \cdot\left(\begin{array}{cc}
0 & \lambda \\
-\mu & y+c
\end{array}\right) \\
& =\left(-\mu \cdot v^{0,1}, \lambda \cdot v^{1,0}+(y+c) \cdot v^{0,1}\right) .
\end{aligned}
$$

We have the graded vector space $\mathcal{G} r=\mathcal{G} r_{-1} \oplus \mathcal{G} r_{1}$ associated with the filtration $W^{\triangle}$. The line bundle $\mathcal{G} r_{-1}$ is obtained by the relation $v^{\dagger 1,0}=-\mu \cdot v^{0,1}$. Thus the first Chern class is -1 . On the other hand, the line bundle $\mathcal{G} r_{1}$ is obtained by the relation $v^{\dagger 0,1}=\lambda \cdot v^{1,0}$. Thus the first Chern class is 1 . Namely the filtered vector bundle $\left(S(O, P), W^{\triangle}\right)$ is a mixed twistor, in this case. 


\subsubsection{Example (the case of $\operatorname{Mod}(l+1, a, C))$}

We consider the case of $\operatorname{Mod}(l+1, a, C)$ for general $l$. Since we know that $\operatorname{Mod}(l+1, a, C)=\operatorname{Sym}^{l} \operatorname{Mod}(2, a, C)$, we obtain a mixed twistor structure also in this case. We see the gluing matrices. We have the canonical frame $\boldsymbol{v}=\left(v^{p, q} \mid p+q=l\right)$ of $\operatorname{Mod}(l+1, a, C)$ over $\mathbf{C}_{\lambda} \times \Delta$. It is also a normalizing frame. The filtration $W^{\triangle}$ is given as follows:

$$
W_{h}^{\triangle}=\left\langle v^{i, l-i} \mid 2 i-l \leq h\right\rangle .
$$

We have the conjugate frame $\boldsymbol{v}^{\dagger}=\left(v^{\dagger p, q} \mid p+q=l\right)$. It is also a normalizing frame. The filtration $W_{h}^{\triangle}$ is given as follows:

$$
W_{h}^{\triangle}=\left\langle v^{\dagger i, l-i} \mid 2 i-l \geq-h\right\rangle
$$

We would like to show that the Chern class of the $h$-th graded part $\mathcal{G} r_{h}$ is $h$. For that purpose, we would like to calculate the transformation matrices $A$ of $\boldsymbol{v}$ and $\boldsymbol{v}^{\dagger}$ determined by the following relation:

$$
v^{\dagger p, l-p}=\sum_{i} A_{i, p} \cdot v^{i, l-i}
$$

However it is not so easy to directly calculate whole of the matrices $A$. We have already known that the filtration $W^{\triangle}$ have to be preserved by the gluing. Then we can derive the following information:

- It implies that $A_{i, p}=0$ if $i>l-p$.

- We also know that $A$ have to be an element of $G L(l+1)$, so that $A_{i, l-i} \neq 0$.

On the other hand, we can show the following by a direct calculation:

- $A_{i, l-i}$ is of the form $c_{i} \cdot \lambda^{2 i-l}$ for some complex number $c_{i}$.

(We only have to use the relations (14), (17) and the orthogonality of the frames $\boldsymbol{v}_{\mid\{0\} \times \Delta}$ and $\left.\boldsymbol{v}_{\mid\{0\} \times \Delta^{\dagger}}^{\dagger}\right)$

Thus the Chern class of $\mathcal{G} r_{h}$ is $h$, namely the filtered vector bundle $(S(O, P), W)$ is a mixed twistor in this case. 


\subsubsection{Some modification of the frames of ${ }^{\diamond} \mathcal{E}_{\mid \mathrm{C}_{\lambda} \times O}$ and ${ }^{\diamond} \mathcal{E}^{\dagger} \mid \mathrm{C}_{\mu} \times O$}

The gluing matrices (49) are divergent when $P \rightarrow O$. (For example, see (50).) We would like to modify our choice of frames of ${ }^{\diamond} \mathcal{E}_{\mid \mathbf{C}_{\lambda} \times O}$ and ${ }^{\diamond} \mathcal{E}^{\dagger} \mid \mathbf{C}_{\mu} \times O$, so that the gluing matrices are convergent for some sequence of points $P_{i} \rightarrow O$.

Let $\boldsymbol{v}$ be a holomorphic frame of $\diamond \mathcal{E}$, compatible with the weight filtration $W^{\triangle}$, over $\mathbf{C}_{\lambda} \times \Delta$. Then the weight filtration $W^{\triangle}$ over $\mathbf{C}_{\lambda} \times O$ is obviously given as follows:

$$
W_{h}^{\triangle}=\left\langle v_{i \mid \mathbf{C}_{\lambda} \times O} \mid \operatorname{deg}^{W^{\triangle}}\left(v_{i}\right) \leq h\right\rangle .
$$

We have the conjugate frame $\boldsymbol{v}^{\dagger}$ of ${ }^{\diamond} \mathcal{E}^{\dagger}$ over $\mathbf{C}_{\mu} \times \Delta$. It is also compatible with the filtration $W^{\triangle}$. Note that $\operatorname{deg}^{W^{\triangle}}\left(v_{i}\right)=-\operatorname{deg}^{W^{\triangle}}\left(v_{i}^{\dagger}\right)$. The weight filtration $W^{\triangle}$ over $\mathbf{C}_{\mu} \times O$ is given as follows:

$$
W_{h}^{\triangle}=\left\langle v_{i \mid \mathbf{C}_{\lambda} \times O}^{\dagger} \mid \operatorname{deg}^{W^{\triangle}}\left(v_{i}^{\dagger}\right) \leq h\right\rangle=\left\langle v_{i \mid \mathbf{C}_{\lambda} \times O}^{\dagger} \mid \operatorname{deg}^{W^{\triangle}}\left(v_{i}\right) \geq-h\right\rangle .
$$

Take holomorphic vector bundles $F:=\bigoplus_{i=1}^{r} \mathcal{O}_{\mathbf{C}_{\lambda}} \boldsymbol{u}_{i}$ over $\mathbf{C}_{\lambda}$. We give the filtration $\mathcal{W}_{F}$ to the bundle $F$, defined as follows:

$$
\mathcal{W}_{F, h}:=\left\langle u_{i} \mid \operatorname{deg}^{W^{\triangle}}\left(v_{i}\right) \leq h\right\rangle .
$$

Similarly we put $F^{\dagger}:=\bigoplus_{i=1}^{r} \mathcal{O}_{\mathbf{C}_{\mu}} \boldsymbol{u}_{i}^{\dagger}$. We give the filtration $\mathcal{W}_{F^{\dagger}}$ defined as follows:

$$
\mathcal{W}_{F^{\dagger}, h}:=\left\langle u_{i}^{\dagger} \mid \operatorname{deg}^{W^{\triangle}}\left(v_{i}^{\dagger}\right) \leq h\right\rangle=\left\langle u_{i}^{\dagger} \mid \operatorname{deg}^{W^{\triangle}}\left(v_{i}\right) \geq-h\right\rangle .
$$

We also put as follows:

$$
U_{h}:=\left\langle u_{i} \mid \operatorname{deg}^{\mathcal{W}}{ }^{\triangle}\left(v_{i}\right)=h\right\rangle, \quad U_{h}^{\dagger}:=\left\langle u_{i}^{\dagger} \mid \operatorname{deg}^{\mathcal{W} \triangleleft}\left(v_{i}^{\dagger}\right)=h\right\rangle .
$$

Then we have $\mathcal{W}_{F, h}=\bigoplus_{l \leq h} U_{l}$ and $\mathcal{W}_{F^{\dagger}, h}=\bigoplus_{l \leq h} U_{l}^{\dagger}$. Note the following obvious lemma.

Lemma 5.7. Let $\boldsymbol{c}=\left(c_{i} \mid i=1, \ldots, r\right)$ be a tuple of nonzero complex numbers. We have the morphism $\eta_{c}:{ }^{\diamond} \mathcal{E}_{\mid \mathbf{C}_{\lambda} \times O} \longrightarrow F$ defined by $v_{i} \longmapsto$ $c_{i} \cdot u_{i}$ for $1 \leq i \leq r$. Then the morphism $\eta_{c}$ preserves the filtrations $W^{\triangle}$ and $\mathcal{W}_{F}$.

Similarly we have the morphism $\eta_{c}^{\dagger}:{ }^{\diamond} \mathcal{E}^{\dagger}{ }_{\mid \mathbf{C}_{\mu} \times O} \longrightarrow F^{\dagger}$ defined by $v_{i}^{\dagger} \longmapsto c_{i} \cdot u_{i}$ for $1 \leq i \leq r$. Then the morphism $\eta^{\dagger}$ preserves the filtrations $W^{\triangle}$ and $\mathcal{W}_{F^{\dagger}}$. 
Proof. Clear from our choices of $\mathcal{W}_{F}$ and $\mathcal{W}_{F^{\dagger}}$.

q.e.d.

For any $P \in \Delta^{*}$, we take the tuple of complex numbers $\boldsymbol{c}(P)=$ $\left(c_{i}(P)\right)$ and $\boldsymbol{c}^{\dagger}(P)=\left(c_{i}^{\dagger}(P)\right)$, given as follows:

$$
\begin{aligned}
c_{i}(P) & :=(-\log |z(P)|)^{k\left(v_{i}\right)}, \\
c_{i}^{\dagger}(P) & :=(-\log |z(P)|)^{k\left(v_{i}^{\dagger}\right)}=(-\log |z(P)|)^{-k\left(v_{i}\right)} .
\end{aligned}
$$

Here we put $2 \cdot k\left(v_{i}\right):=\operatorname{deg}^{W^{\triangle}}\left(v_{i}\right)$ and $2 \cdot k\left(v_{i}^{\dagger}\right):=\operatorname{deg}^{W^{\triangle}}\left(v_{i}^{\dagger}\right)$. Then we obtain the following isomorphisms preserving the filtrations:

$$
\begin{aligned}
\eta_{\boldsymbol{c}(P)}:\left({ }^{\diamond} \mathcal{E}_{\mid \mathbf{C}_{\lambda} \times O}, W\right) & \simeq\left(F, \mathcal{W}_{F}\right), \\
\eta_{\boldsymbol{c}^{\dagger}(P)}:\left({ }^{\diamond} \mathcal{E}^{\dagger}{ }_{\mid \mathbf{C}_{\mu} \times O}, W\right) & \simeq\left(F^{\dagger}, \mathcal{W}_{F^{\dagger}}\right) .
\end{aligned}
$$

Then the gluing morphism $\Psi(O, P)$ for the vector bundle $S(O, P)$ induces the isomorphism $g(P):\left(F, \mathcal{W}_{F}\right)_{\mid \mathbf{C}_{\lambda}^{*}} \simeq\left(F^{\dagger}, \mathcal{W}_{F^{\dagger}}\right)_{\mid \mathbf{C}_{\mu}^{*}}$. The filtered vector bundle $\left(S(O, P), W^{\triangle}\right)$ is naturally isomorphic to the filtered vector bundle obtained from $\left(F, \mathcal{W}_{F}\right),\left(F^{\dagger}, \mathcal{W}_{F^{\dagger}}\right)$ and the gluing $g(P)$.

We have the diagonal matrix $L(P)$ whose $(i, i)$-component is $c_{i}(P)=$ $(-\log |z(P)|)^{k\left(v_{i}\right)}$. We also have the diagonal matrix $L^{\dagger}(P)$ whose $(i, i)$ component is $c_{i}^{\dagger}(P)=(-\log |z(P)|)^{k\left(v_{i}^{\dagger}\right)}=\left.(-\log \mid z(P))\right|^{-k\left(v_{i}\right)}$. The matrix $L^{\dagger}(P)$ is the inverse of $L(P)$. Let $A(\lambda, P)$ be an element of $\Gamma\left(\mathbf{C}_{\lambda}^{*}, G L(r)\right)$ given in (49).

Lemma 5.8. The gluing $g(P)$ is represented by $L(P) \cdot A(\lambda, P)$. $L^{\dagger}(P)^{-1}$ with respect to the frames $\boldsymbol{u}$ and $\boldsymbol{u}^{\dagger}$.

Proof. Since $A(\lambda, P)$ represents $\Psi(O, P)$ with respect to the frames $\boldsymbol{v}_{\mid \mathbf{C}_{\lambda} \times O}$ and $\boldsymbol{v}_{\mid \mathbf{C}_{\mu} \times O}$, the claim is clear from our choices of $\eta_{\boldsymbol{c}}$ and $\eta_{\boldsymbol{c}^{\dagger}}^{\dagger}$. q.e.d.

In the following, we assume that we take $\boldsymbol{e}=\boldsymbol{v}_{\mid\{0\} \times \Delta^{*}}$ for the construction of $A(\lambda)$.

\subsubsection{The statement and an outline of a proof}

We will prove the following theorem.

Theorem 5.1 (A limiting mixed twistor theorem). Let $\left(E, \bar{\partial}_{E}, \theta, h\right)$ be a tame nilpotent harmonic bundle with parabolic structure over $\Delta^{*}$. For any open set $U$ containing $O$, there is a point $P \in U \cap \Delta^{*}$ such that the filtered vector bundle $\left(S(O, P), W^{\triangle}\right)$ gives a mixed twistor structure. 
An outline of the proof of the theorem is as follows:

1. We can take some sequence of the points $\left\{P_{i}\right\}$ converging to $O$, such that the corresponding sequence of the gluing functions $\left\{g\left(P_{i}\right)\right\}$ converge to the gluing function $g_{\infty}$ (Subsubsection 5.3.6).

2. We have the filtered vector bundle $\left(S_{\infty}, \mathcal{W}\right)$ obtained from $\left(F, \mathcal{W}_{F}\right)$, $\left(F^{\dagger}, \mathcal{W}_{F^{\dagger}}\right)$ and $g_{\infty}$. We will see that $\left(S_{\infty}, \mathcal{W}\right)$ is a mixed twistor (Subsubsection 5.3.7).

3. Consider the condition that a filtered vector bundle is a mixed twistor. We can see that the condition is open. Since the sequence of the filtered vector bundles $\left(S\left(O, P_{i}\right), W^{\triangle}\right)$ converge to $\left(S_{\infty}, \mathcal{W}\right)$ in a sense, we can conclude that they are mixed twistors for sufficiently large $i$ (Subsubsection 5.3.8).

Before entering the proof, we state an obvious corollary.

Corollary 5.1. Let $P$ be any point of $\Delta^{*}$. The Chern class $c_{1}\left(\mathcal{G} r_{h}^{\triangle}\right)$ is $h \cdot \operatorname{rank}\left(\mathcal{G} r_{h}^{\triangle}\right)$.

Proof. If $\left(S(O, P), W^{\triangle}\right)$ is mixed twistor, the claim is clear. Since the Chern class is an topological invariant, we obtain the result. q.e.d.

Remark 5.1. See Note Added in Proof in the last of the paper.

\subsubsection{Convergency of the gluing functions}

We only have to investigate the convergency of the sequence $\left\{L\left(P_{l}\right)\right.$. $\left.A\left(P_{l}\right) \cdot L^{\dagger}\left(P_{l}\right)^{-1}\right\}$ for some sequence $\left\{P_{l}\right\}$. We decompose $L(P) \cdot A(P)$. $L^{\dagger}(P)^{-1}$ as follows:

$$
\begin{aligned}
& \left(L(P) \cdot \Gamma(\lambda, O) \cdot \Gamma(\lambda, P)^{-1} \cdot L(P)^{-1}\right) \\
& \cdot\left(L(P) \cdot B(\lambda, P)^{-1} L(P)^{-1}\right) \\
& \cdot\left(L(P) \cdot \overline{H(h, \boldsymbol{e})}^{-1}(P) \cdot L^{\dagger}(P)^{-1}\right) \\
& \cdot\left(L^{\dagger}(P) \cdot B^{\dagger}(\mu, P) \cdot L^{\dagger}(P)^{-1}\right) \\
& \cdot\left(L^{\dagger}(P) \cdot \Gamma^{\dagger}(\mu, P) \cdot \Gamma^{\dagger}(\mu, O)^{-1} \cdot L^{\dagger}(P)^{-1}\right) \text {. }
\end{aligned}
$$

Lemma 5.9. Let $R$ be a real number such that $0<R<1$. On the region $T(R)=\left\{\lambda \in \mathbf{C}_{\lambda}|R<| \lambda \mid<R^{-1}\right\}$, the sequence $\left\{L\left(P_{l}\right) \cdot \Gamma(\lambda, O)\right.$. $\left.\Gamma\left(\lambda, P_{l}\right)^{-1} \cdot L\left(P_{l}\right)^{-1}\right\}$ converges to the identity matrix, with respect to the sup norms, for any sequence $\left\{P_{l}\right\}$ converging to $O$. 
Proof. The sequence $\left\{\Gamma(\lambda, O) \cdot \Gamma\left(\lambda, P_{l}\right)^{-1}\right\}$ converges to the identity matrix for any sequence $\left\{P_{l}\right\}$. In fact, we have an equality $\mid \Gamma(\lambda, O)$. $\Gamma(\lambda, P)^{-1}-1|\leq C \cdot| z(P) \mid$ over the region $T(R)$, for some positive constant $C$.

q.e.d.

Lemma 5.10. Let $\left\{P_{l}\right\}$ be a sequence of points of $\Delta^{*}$, converging to $O$. Then we can take a subsequence $\left\{P_{l_{i}}\right\}$ such that the corresponding sequence $\left\{L\left(P_{l_{i}}\right) \cdot \overline{H(h, e)}^{-1}\left(P_{l_{i}}\right) \cdot L^{\dagger}\left(P_{l_{i}}\right)^{-1}\right\}$ converges to a positive definite hermitian matrix $M$.

Proof. Recall we assume $\boldsymbol{e}=\boldsymbol{v}_{\mid\{0\} \times \Delta^{*}}$. We put $e_{i}^{\prime}:=(-\log |z|)^{-k\left(e_{i}\right)}$. $e_{i}$ for $i=1, \ldots, r$. Then the frame $\boldsymbol{e}^{\prime}=\left(e_{1}^{\prime}, \ldots, e_{r}^{\prime}\right)$ of $\mathcal{E}^{0}$ over $\Delta^{*}$ is adapted. We have the following equality:

$$
H\left(h, \boldsymbol{e}^{\prime}\right)=L(P)^{-1} \cdot H(h, \boldsymbol{e}) \cdot L(P)^{-1}=L^{\dagger}(P) \cdot H(h, \boldsymbol{e}) \cdot L(P)^{-1} .
$$

Thus we are done.

q.e.d.

Let us investigate the convergency of $\left\{L(P)^{-1} \cdot B(\lambda, P) \cdot L(P)\right\}$. For that purpose, we take a model bundle. We put $V={ }^{\diamond} \mathcal{E}^{0} \mid O$ and $N=\operatorname{Res}(\theta)$. Then we have a model bundle $\left(E_{0}, \theta_{0}, h_{0}\right)=E(V, N)$ over $\Delta^{*}$. We denote the deformed holomorphic bundle of $\left(E_{0}, \theta_{0}, h_{0}\right)$ by $\left(\mathcal{E}_{0}, \mathbb{D}_{0}, h_{0}\right)$. We have the canonical frame $\boldsymbol{v}_{0}$ of ${ }^{\diamond} \mathcal{E}_{0}$ over $\mathbf{C}_{\lambda} \times \Delta$. We can assume $\operatorname{deg}^{W}\left(v_{0}\right)=\operatorname{deg}^{W}\left(v_{i}\right)$.

We put $\boldsymbol{e}_{0}:=\boldsymbol{v}_{0 \mid\{0\} \times \Delta}$. Then the frames $\boldsymbol{e}_{0}$ and $\boldsymbol{e}$ induce the isomorphism $F$ of the holomorphic bundles ${ }^{\diamond} \mathcal{E}_{0}^{0}$ and ${ }^{\diamond} \mathcal{E}_{0}$, such that $F_{\mid O}{ }^{\circ}$ $\operatorname{Res}\left(\theta_{0}\right)=\operatorname{Res}(\theta) \circ F_{\mid O}$. The morphism $F$ induces the holomorphic isomorphism $F: \mathcal{E}_{0}^{0} \longrightarrow \mathcal{E}_{0}$ over $\Delta^{*}$, such that $F$ and the inverse $F^{-1}$ are bounded with respect to the metrics $h_{0}$ and $h$.

Since we have $\mathcal{E}_{0}=p_{\lambda}^{-1} \mathcal{E}_{0}^{0}$ and $\mathcal{E}=p_{\lambda}^{-1} \mathcal{E}_{0}$ by our definition, we obtain a $C^{\infty}$-isomorphism $\mathcal{I}: \mathcal{E}_{0} \longrightarrow \mathcal{E}$ over $\mathbf{C}_{\lambda} \times \Delta^{*}$. The morphism $\mathcal{I}$ and the inverse $\mathcal{I}^{-1}$ are bounded with respect to the metrics $h$ and $h_{0}$ by our construction.

We have the $G L(r)$-valued function $I$ determined by $\mathcal{I}\left(\boldsymbol{v}_{0}\right)=\boldsymbol{v} \cdot I$. We have the $G L(r)$-valued function $B_{0}$ determined by $\boldsymbol{v}_{0}=p_{\lambda}^{-1}\left(\boldsymbol{e}_{0}\right) \cdot B_{0}$.

Lemma 5.11. We have the relation $B=B_{0} \cdot I^{-1}$.

Proof. We have $\boldsymbol{v}=p_{\lambda}^{-1}(\boldsymbol{e}) \cdot B$. On the other hand, we have the following:

$$
\boldsymbol{v}=\mathcal{I}\left(\boldsymbol{v}_{0}\right) \cdot I^{-1}=\mathcal{I}\left(p_{\lambda}^{-1} \boldsymbol{e}_{0}\right) \cdot B_{0} \cdot I^{-1}
$$


By our choice of $\mathcal{I}$, we have $\mathcal{I}\left(p_{\lambda}^{-1} \boldsymbol{e}_{0}\right)=p_{\lambda}^{-1} \boldsymbol{e}$. Thus we are done. q.e.d.

Hence we have the following:

$$
\begin{aligned}
& L(P) \cdot B(\lambda, P) \cdot L(P)^{-1} \\
& =\left(L(P) \cdot B_{0}(\lambda, P) \cdot L(P)^{-1}\right) \times\left(L(P) \cdot I^{-1}(\lambda, P) \cdot L(P)^{-1}\right) .
\end{aligned}
$$

For the model bundle, we have already known the behaviour of the transformation matrices $B_{0}(P, \lambda)$ when $P \rightarrow 0$.

Lemma 5.12. When $P \rightarrow O$, the sequence $\left\{L(P) \cdot B_{0}(\lambda, P)\right.$. $\left.L(P)^{-1}\right\}$ converges to a $G L(r)$-valued holomorphic function $\bar{B}(\lambda)=$ $\left(\bar{B}_{i j}(\lambda)\right)$ over $\mathbf{C}_{\lambda}$ satisfying the following:

- We put $k\left(v_{i}\right):=2^{-1} \cdot \operatorname{deg}^{W}\left(v_{i}\right)=2^{-1} \cdot \operatorname{deg}^{W}\left(v_{0 i}\right)$. Then we have the following:

$$
\bar{B}_{i j}(\lambda)= \begin{cases}0 & \text { if } k\left(v_{i}\right)<k\left(v_{j}\right) \\ & \text { or if } k\left(v_{j}\right)-k\left(v_{i}\right) \\ & \text { is not an integer }), \\ b_{i j} \cdot \lambda^{k\left(v_{i}\right)-k\left(v_{j}\right)},\left(b_{i j} \in \mathbf{C}\right) & \text { (if } k\left(v_{j}\right)-k\left(v_{i}\right) \\ & \text { is an integer }) .\end{cases}
$$

- The matrices $\bar{B}(h, h):=\left(b_{i j} \mid \operatorname{deg}^{W}\left(v_{i}\right)=\operatorname{deg}^{W}\left(v_{j}\right)=h\right)$ are invertible for any $h$.

- The matrices $\bar{B}(h,-h):=\left(b_{i j} \mid \operatorname{deg}^{W}\left(v_{i}\right)=h, \operatorname{deg}^{W}\left(v_{j}\right)=-h\right)$ are invertible for any $h \leq 0$.

A similar convergence holds for the sequence $L^{\dagger}(P) B_{0}^{\dagger}(P) L^{\dagger}(P)^{-1}$, when $P \rightarrow O$.

Proof. The claims can be checked directly. (See Corollary 3.4.)

We put $C(P):=L(P) \cdot I(\lambda, P) \cdot L(P)^{-1}$, which is an element of $C^{\infty}\left(\mathbf{C}_{\lambda} \times \Delta^{*}, G L(r)\right)$.

Lemma 5.13. Let $K$ be a compact subset of $\mathbf{C}_{\lambda}$. Then $C(P)$ and the inverse $C(P)^{-1}$ are bounded over $K \times \Delta^{*}$.

Proof. We put $v_{i}^{\prime}:=(-\log |z|)^{-k\left(v_{i}\right)} \cdot v_{i}$. Then we obtain $C^{\infty}$-frame $\boldsymbol{v}^{\prime}=\left(v_{i}^{\prime}\right)$ of $\mathcal{E}$ over $\mathbf{C}_{\lambda} \times \Delta^{*}$. We also put $v_{0 i}^{\prime}=(-\log |z|)^{-k\left(v_{0} i\right)} \cdot v_{0 i}$, 
and then we obtain the $C^{\infty}$-frame $\boldsymbol{v}_{0}^{\prime}$ of $\mathcal{E}_{0}$. For any compact subset $K \subset \mathbf{C}_{\lambda}$, the frames $\boldsymbol{v}^{\prime}$ and $\boldsymbol{v}_{0}^{\prime}$ are adapted over $K \times \Delta^{*}$, for the metrics $h$ and $h_{0}$ respectively. Since $\mathcal{I}$ and the inverse $\mathcal{I}^{-1}$ are bounded over $K \times \Delta^{*}$, the $C^{\infty}$-frame $\mathcal{I}\left(\boldsymbol{v}_{0}^{\prime}\right)$ is adapted for the metric $h$. Then the transformation matrices between $\boldsymbol{v}^{\prime}$ and $\mathcal{I}\left(\boldsymbol{v}_{0}^{\prime}\right)$ are bounded. Now we have the relation $\mathcal{I}\left(\boldsymbol{v}_{0}^{\prime}\right)=\boldsymbol{v}^{\prime} \cdot C$ by our construction. Thus $C$ and the inverse $C^{-1}$ are bounded over $K \times \Delta^{*}$.

q.e.d.

We have the following immediate corollary.

Corollary 5.2. Let $\left\{P_{l}\right\}$ be a sequence of points in $\Delta^{*}$ converging to $O$. Then we can take a subsequence $\left\{P_{l_{i}}\right\}$ such that the corresponding sequence $\left\{C\left(P_{l}\right)\right\}$ converges to a $G L(r)$-valued holomorphic function $\bar{C}$ with respect to the sup norm, over the region $\Delta_{\lambda}(R)$ for any $0<R<1$.

Then we have already known the existence of the sequence $\left\{P_{l}\right\}$ such that $\left\{L\left(P_{l}\right) \cdot A\left(\lambda, P_{l}\right) \cdot L^{\dagger}\left(P_{l}\right)^{-1}\right\}$ converges to a $G L(r)$-valued holomorphic functions over the region $T(R)=\left\{\lambda|R<| \lambda \mid<R^{-1}\right\}$. However, we need a better sequence.

Lemma 5.14. There is a sequence $\left\{P_{l}\right\}$ satisfying the following:

- The sequence $\left\{C\left(P_{l}\right)\right\}$ converges to $G L(r)$-valued holomorphic function $\bar{C}$ over $\mathbf{C}_{\lambda}$.

- The $(i, j)$-components $\bar{C}_{i j}$ are 0 if $\operatorname{deg}\left(v_{i}\right) \neq \operatorname{deg}\left(v_{0 j}\right)$. In other words, $\bar{C}$ preserves the decomposition $F=\bigoplus_{h} U_{h}$.

Proof. We only have to see that we can take a sequence $\left\{P_{l}\right\}$ satisfying $C_{i j}\left(P_{l}\right) \rightarrow 0$ for any pair $(i, j)$ such that $\operatorname{deg}\left(v_{0 j}\right) \neq \operatorname{deg}\left(v_{i}\right)$.

Assume that $\operatorname{deg}\left(v_{0 j}\right) \neq \operatorname{deg}\left(v_{i}\right)$. Due to Lemma 4.9 and Lemma 4.19, we obtain the following finiteness:

$$
\left\|\int_{|\lambda|<R}\left|C_{i j}(\lambda, z)\right| \cdot|d \lambda \cdot d \bar{\lambda}|\right\|_{W}=\int_{|\lambda|<R}\left\|C_{i j}(\lambda, z)\right\|_{W} \cdot|d \lambda \cdot d \bar{\lambda}|<\infty .
$$

Thus we can take a sequence $\left\{P_{l}\right\}$ such that the sequence

$$
\left\{\int_{|\lambda|<R}\left|C_{i j}\left(\lambda, P_{l}\right)\right| \cdot|d \lambda \cdot d \bar{\lambda}|\right\}
$$

converges to 0 for any $R>0$ Since the components $C_{i j}\left(\lambda, P_{l}\right)$ depend on $\lambda$ holomorphically, the sequence $\left\{C_{i j}\left(\lambda, P_{l}\right)\right\}$ converges to 0 on $\Delta_{\lambda}(R)$ for any $R>0$.

In all, we obtain the following: 
Proposition 5.3. There exists a sequence $\left\{P_{l}\right\}$ of points in $\Delta^{*}$ converging to $O$ satisfying the following:

- The corresponding sequence $\left\{L(P) \cdot A(\lambda, P) \cdot L^{\dagger}(P)^{-1}\right\}$ converges to a holomorphic $G L(r)$-valued function $\bar{A}$ on $\mathbf{C}_{\lambda}^{*}$. The convergence is with respect to the sup norms on $T(R):=\left\{\lambda \in \mathbf{C}_{\lambda} \mid R<\right.$ $\left.|\lambda|<R^{-1}\right\}$ for any $0<R<1$.

- We have the decomposition of $\bar{A}$ into the product:

$$
\bar{A}=\bar{C} \cdot \bar{B}_{0}^{-1} \cdot M \cdot \bar{B}_{0}^{\dagger} \cdot \bar{C}^{\dagger-1} .
$$

- $M$ is a positive definite hermitian matrix. It is independent of $\lambda$ and $\mu$.

- $\bar{B}_{0}$ is a holomorphic $G L(r)$-valued function defined over $\mathbf{C}_{\lambda}$. It is given in Lemma 5.12.

- $\bar{C}$ is a holomorphic $G L(r)$-valued function defined over $\mathbf{C}_{\lambda}$. It preserves the decomposition $F=\bigoplus_{h} U_{h}$.

- $\bar{B}_{0}^{\dagger}$ and $\bar{C}^{\dagger}$ are $G L(r)$-valued holomorphic functions defined over $\mathbf{C}_{\mu}$. They are given as follows:

$$
\bar{B}_{0}^{\dagger}(\mu)={ }^{t}{\overline{\bar{B}_{0}(-\bar{\lambda})}}^{-1}, \quad \bar{C}^{\dagger}(\mu)={ }^{t} \overline{\bar{C}(-\bar{\lambda})}^{-1} .
$$

- Similar things hold for the exterior products $\bigwedge^{l}(E, \theta, h)$.

Proof. We have already seen the first five properties. The last two properties are clear from our construction. Note that the transformation matrices for $S\left(O, P, \bigwedge^{l}(E, \theta, h)\right)$ is obtained from the transformation matrices of $S(O, P,(E, \theta, h))$, by some standard linear algebraic procedures.

q.e.d.

\subsubsection{The property of $g_{\infty}$}

Let $g_{\infty}$ denote the gluing given by the matrices $\bar{A}$. From the filtered vector bundles $\left(F, \mathcal{W}_{F}\right),\left(F^{\dagger}, \mathcal{W}_{F^{\dagger}}\right)$ and the gluing $g_{\infty}$, we obtain the filtered vector bundle. We denote it by $\left(S^{(\infty)}, \mathcal{W}^{(\infty)}\right)$. We denote the associated graded vector bundle by $\bigoplus_{h} \mathcal{G} r_{h}$.

Proposition 5.4. The filtered vector bundle $\left(S^{(\infty)}, \mathcal{W}^{(\infty)}\right)$ is a mixed twistor. Namely $\mathcal{G} r_{h}$ is isomorphic to a direct sum of $\mathcal{O}_{\mathbb{P}^{1}}(h)$. 
Proof. First we see the following:

Lemma 5.15. We only have to consider the transformation given by $\bar{B}^{-1} \cdot M \cdot \bar{B}^{\dagger}$.

Proof. The matrix valued function $\bar{C}$ and the inverse are holomorphic for the variable $\lambda$ over $\mathbf{C}_{\lambda}$. Moreover they preserve the decomposition $\bigoplus U_{h}$. In particular, they preserve the filtration $\mathcal{W}_{F}$ over $\mathbf{C}_{\lambda}$. Thus we can ignore $\bar{C}$ to see $\mathcal{G} r_{h}$. Similarly we can ignore $\bar{C}^{\dagger}$. q.e.d.

We denote the associated graded vector bundle of $\mathcal{W}_{F}$ and $\mathcal{W}_{F^{\dagger}}$ by $\mathcal{G} r_{F}$ and $\mathcal{G} r_{F^{\dagger}}$. Since $\bar{B}^{-1} \cdot M \cdot \bar{B}^{\dagger}$ preserves the filtrations $\mathcal{W}_{F}$ and $\mathcal{W}_{F^{\dagger}}$, we obtain the morphisms of the graded parts. We denote them as follows:

$$
G r_{l}\left(\bar{B}^{-1} \cdot M \cdot \bar{B}^{\dagger}\right): \mathcal{G} r_{l}^{\mathcal{W}_{F}} \longrightarrow \mathcal{G} r_{l}^{\mathcal{W}_{F}^{\dagger}}
$$

Let $b(W)$ be the bottom number of the filtration $\mathcal{W}^{(\infty)}$.

Lemma 5.16. The morphism

$$
\operatorname{Gr}_{b(W)}\left(\bar{B}^{-1} M \bar{B}^{\dagger}\right): \mathcal{G} r_{b(W)}^{\mathcal{W}_{F}} \longrightarrow \mathcal{G} r_{b(W)}^{\mathcal{W}_{F}^{\dagger}}
$$

is of the form $\lambda^{-b(W)} \times \Phi_{b(W)}(\mu)$, where $\Phi_{b(W)}$ is holomorphic and invertible on $\mathbf{C}_{\mu}$. In particular, $\mathcal{G} r_{b(W)}$ is a pure twistor of weight $b(W)$.

Proof. We can regard the matrices $\bar{B}^{-1}$ and $\bar{B}^{\dagger}$ as the endomorphisms of $F$ and $F^{\dagger}$. And the hermitian matrix $M$ can be regarded as the transformation of $\boldsymbol{u}=\left(u_{i}\right)$ to $\boldsymbol{u}^{\dagger}=\left(u_{i}^{\dagger}\right)$. We denote the submatrices $\left(\left(\bar{B}^{-1}\right)_{i j} \mid \operatorname{deg}\left(u_{i}\right)=x, \operatorname{deg}\left(u_{j}\right)=y\right)$ by $\bar{B}^{-1}(x, y)$. Similarly we put as follows:

$$
\begin{aligned}
\bar{B}^{\dagger}(x, y) & :=\left(\bar{B}_{i j}^{\dagger} \mid \operatorname{deg}\left(u_{i}^{\dagger}\right)=x, \operatorname{deg}\left(u_{j}^{\dagger}\right)=y\right), \\
M(x, y) & :=\left(M_{i j} \mid \operatorname{deg}\left(u_{i}\right)=x, \operatorname{deg}\left(u_{j}^{\dagger}\right)=y\right), \\
\left(\bar{B}^{-1} \cdot M \cdot \bar{B}^{\dagger}\right)(x, y) & :=\left(\left(\bar{B}^{-1} \cdot M \cdot \bar{B}^{\dagger}\right)_{i j} \mid \operatorname{deg}\left(u_{i}\right)=x, \operatorname{deg}\left(u_{j}^{\dagger}\right)=y\right) .
\end{aligned}
$$

We will calculate $\left(\bar{B}^{-1} \cdot M \cdot \bar{B}^{\dagger}\right)(b(W), b(W))$. Note the following obvious lemma.

Lemma 5.17. We have $\bar{B}^{\dagger}(x, y)=0$ for any $x>y$. In particular $\bar{B}^{\dagger}(x, b(W))=0$ if $x \neq b(W)$. 
Then we obtain the following equalities:

$$
\begin{aligned}
& \left(\bar{B}^{-1} \cdot M \cdot \bar{B}^{\dagger}\right)(b(W), b(W)) \\
& =\sum_{y} \bar{B}^{-1}(b(W), y) \cdot M(y, b(W)) \cdot \bar{B}^{\dagger}(b(W), b(W)) \\
& =\bar{B}^{-1}(b(W),-b(W)) \cdot M(-b(W), b(W)) \cdot \bar{B}^{\dagger}(b(W), b(W)) \\
& \quad+\sum_{y<-b(W)} \bar{B}^{-1}(b(W), y) \cdot M(y, b(W)) \cdot \bar{B}^{\dagger}(b(W), b(W)) .
\end{aligned}
$$

If follows from Lemma 5.12 that the function $\bar{B}^{-1}(b(W),-b(W))$ is of the form $\lambda^{-b(W)} Q_{1}$ where $Q_{1}$ is an element of $G L_{r^{\prime}}(\mathbf{C})$. Here $r^{\prime}$ denotes $\operatorname{dim} \mathcal{G} r_{b(W)}$. The matrix $M(-b(W), b(W))$ is positive hermitian. Note that we have the equality $\operatorname{deg}\left(u_{i}\right)=-\operatorname{deg}\left(u_{i}^{\dagger}\right)$. Moreover $\bar{B}^{\dagger}(b(W), b(W))$ is an element of $G L_{r^{\prime}}(\mathbf{C})$. Thus the first term in the right-hand side $\bar{B}^{-1}(b(W),-b(W)) \cdot M(-b(W), b(W)) \cdot \bar{B}^{\dagger}(b(W), b(W))$ is of the form $\lambda^{-b(W)} Q_{2}$ where $Q_{2}$ is an element of $G L_{r^{\prime}}(\mathbf{C})$.

If follows from Lemma 5.12 that the function $\bar{B}^{-1}(b(W), y) \quad(y<$ $-b(W))$ is of the form $\lambda^{(-b(W)+y) / 2} S_{y}$ where $S_{y}$ denotes a matrix with C-coefficient and $S_{y}$ is 0 unless $b(W)-y$ is even. Also $M(y, b(W))$ is a matrix with $\mathbf{C}$-coefficient. Thus we have the following equality:

$$
\begin{aligned}
& \left(\bar{B}^{-1} M \bar{B}^{\dagger}\right)(b(W), b(W))=\lambda^{-b(W)} \times \Phi_{b(W)}(\mu) \\
& \Phi_{b(W)}(\mu)=Q_{2}+\sum_{i>0} \mu^{i} S_{i}^{\prime} .
\end{aligned}
$$

Here $S_{i}^{\prime}(i>0)$ denote the matrices with $\mathbf{C}$-coefficient. The function $\Phi_{b(W)}(\mu)$ is holomorphic and invertible over $\mathbf{C}_{\mu}$.

In particular, $\Phi_{b(W)}(\mu)$ is just a change of the trivialization of $\mathcal{G} r_{b(W)}^{\mathcal{W}_{F}^{\dagger}}$ over $\mathbf{C}_{\mu}$. As a result, we can conclude that $\mathcal{G} r_{b(W)}$ is a pure twistor of weight $b(W)$. Thus the proof of Lemma 5.16 is completed. q.e.d.

Then we will show the following claim by using an induction on $h$.

$\left(\boldsymbol{P}_{\boldsymbol{h}}\right)$ For any $l<h$, the graded part $\mathcal{G} r_{l}$ is a pure twistor of weight $l$.

We assume that $P_{h-1}$ holds and show that $P_{h}$ holds. By assumption, $\mathcal{G} r_{l}$ are pure twistor of weight $l$. In particular the first Chern class of $\operatorname{det}\left(\mathcal{G} r_{l}\right)$ is $l \cdot \operatorname{rank}\left(\mathcal{G} r_{l}\right)$. 
We put $R=\operatorname{rank} \mathcal{W}_{h-1}^{(\infty)}+1$. Note that we have already seen that the bottom part is a pure twistor of an appropriate weight for any tame nilpotent harmonic bundle with nilpotent residues. In particular, the bottom part of the filtered vector bundle $\bigwedge^{R}\left(S^{(\infty)}, \mathcal{W}^{(\infty)}\right)$ is pure twistor of the following weight:

$$
b_{0}:=\sum_{l=1}^{h-1} l \cdot \operatorname{rank} \mathcal{G} r_{l}+h=\sum_{l=1}^{h-1} c_{1}\left(\operatorname{det} \mathcal{G} r_{l}\right)+h=c_{1}\left(\operatorname{det} \mathcal{W}_{h-1}^{(\infty)}\right)+h .
$$

There is the natural isomorphism:

$$
\mathcal{G} r_{b_{0}}\left(\bigwedge^{R}\left(S^{(\infty)}, \mathcal{W}^{(\infty)}\right)\right) \simeq \operatorname{det}\left(\mathcal{W}_{h-1}^{(\infty)}\right) \otimes \mathcal{G} r_{h}
$$

Thus we can conclude that $\mathcal{G} r_{h}$ is a pure twistor of weight $h$.

Hence the proof of Proposition 5.4 is completed.

\subsubsection{The end of the proof of a limiting mixed twistor theo- rem}

For a positive number $R$ such that $1<R<\infty$, we put $T(R):=\{z \in$ $\left.\mathbf{C}\left|R^{-1}<\right| z \mid<R\right\}$. For any holomorphic function $g: T(R) \longrightarrow G L(n)$, we can naturally associate the holomorphic vector bundle of rank $n$, which we denote by $V(g)$. We denote the set of holomorphic function $g: T(R) \longrightarrow G L(n)$ such that $c_{1}(V(g))=0$ by $\mathcal{C}_{0}$. We have the subset $\mathcal{C}_{\text {triv }}=\left\{g \in \mathcal{C}_{0} \mid V(g)\right.$ trivial $\}$.

Lemma 5.18. $\quad \mathcal{C}_{\text {triv }}$ is open with respect to the topology given by the sup norm, i.e., if a sequence $\left\{g_{i} \in \mathcal{C}_{0}\right\}$ converges to an element $g \in \mathcal{C}_{\text {tirv }}$, then $g_{i} \in \mathcal{C}_{\text {triv }}$ for any sufficiently large $i$.

Proof. We can translate the sequence of the gluing $g_{i}$ to the sequence of holomorphic structures $\bar{\partial}_{i}$ on a $C^{\infty}$-vector bundle $E$ such that $c_{1}(E)=0$. The sequence $\bar{\partial}_{i}$ converges to a holomorphic structure $\bar{\partial}$ in $L_{l}^{p}$ for any $l$, such that $(E, \bar{\partial})$ is isomorphic to the trivial bundle. Then we have to show that $\bar{\partial}_{i}$ gives the holomorphic structure which is holomorphically trivial for any sufficiently large $i$. It is a consequence of the vanishing $H^{1}\left(\mathbb{P}^{1}, \mathcal{O}\right)=0$.

q.e.d.

By Lemma 5.18, we obtain the open-ness of the condition that a filtered vector bundle is a mixed twistor. We have already known that the sequence of the gluings $\left\{g\left(P_{l}\right)\right\}$ converges to a gluing $g(P)$, which gives 
a mixed twistor. Then we can conclude that $g\left(P_{l}\right)$ gives a mixed twistor if $l$ is sufficiently large. Thus the proof of Theorem 5.1 is completed.

q.e.d.

\subsection{Higher dimensional case of a limiting mixed twistor theorem}

\subsubsection{The morphisms induced by the residues}

We put $X=\Delta^{n}, D_{i}=\left\{z_{i}=0\right\}$, and $D=\bigcup_{i=1}^{l} D_{i}$ for some $l \leq n$.

Consider the tame nilpotent harmonic bundle $\left(E, \bar{\partial}_{E}, \theta, h\right)$ with trivial parabolic structure over $X-D$. Take a point $Q \in D$. We put $I=\left\{i \in \underline{l} \mid Q \in D_{i}\right\}$. Take a point $P$ and consider $S(O, P)$ over $\mathbb{P}^{1}$ for $\left(E, \bar{\partial}_{E}, \theta, h\right)$. We have the nilpotent maps induced by the residues of $\mathbb{D}$ and $\mathbb{D}^{\dagger}$ at $\mathcal{D}_{i}$ and $\mathcal{D}_{i}^{\dagger}$ respectively:

$$
N_{i}^{\triangle}: S(Q, P) \longrightarrow S(Q, P) \otimes \mathcal{O}_{\mathbb{P}}(2), \quad(i \in I) .
$$

For a tuple $\boldsymbol{a}=\left(a_{i} \mid i \in I\right) \in \mathbf{C}^{I}$, we put $N^{\triangle}(\boldsymbol{a}):=\sum_{i \in I} a_{i} \cdot N_{i}^{\triangle}$.

Lemma 5.19. Assume that all of $a_{i}$ are positive integers.

- The conjugacy classes of $N^{\triangle}(\boldsymbol{a})_{\mid \lambda}$ are independent of the choice of $\lambda \in \mathbb{P}^{1}$.

- Let $W^{\triangle}(\boldsymbol{a})$ denote the weight filtration of $N^{\triangle}(\boldsymbol{a})$. For an appropriate point $P$, the filtered vector bundle $\left(S(Q, P), W^{\triangle}(\boldsymbol{a})\right)$ is a mixed twistor.

Proof. Consider the embedding $\varphi: \Delta \longrightarrow X$ given as follows:

$$
z_{i}(\varphi(t))= \begin{cases}t^{a_{i}} & (i \in I) \\ z_{i}(Q) \neq 0 & (i \notin I) .\end{cases}
$$

We denote the origin of $\Delta$ by $O$. Take a point $\widetilde{P} \in \Delta$ such that $\varphi(\widetilde{P})=$ $P$.

We obtain the harmonic bundle $\varphi^{*}\left(E, \bar{\partial}_{E}, \theta, h\right)$ over $\Delta^{*}$. By our construction and the functoriality (Proposition 5.2), the residue $\operatorname{Res}\left(\varphi^{*} \mathbb{D}\right.$ ) is isomorphic to $N^{\triangle}(\boldsymbol{a})_{\mid \mathbf{C}_{\lambda}}$. Thus the conjugacy classes of $N^{\triangle}(\boldsymbol{a})_{\mid \mathbf{C}_{\lambda}}$ are independent of a choice of $\lambda \in \mathbf{C}_{\lambda}$. Similarly the conjugacy class of $N^{\triangle}(\boldsymbol{a})_{\mid \mathbf{C}_{\mu}}$ are independent of a choice of $\mu \in \mathbf{C}_{\mu}$. Moreover, the filtration $W^{\triangle}(\boldsymbol{a})$ gives a mixed twistor if we take an appropriate point $\widetilde{P}$ of $\Delta^{*}$, which is a consequence of Theorem 5.1 .

q.e.d. 
We can take a general $\boldsymbol{a}$, in the sense of 2.14, from $\mathbf{Q}_{>0}^{I}$. Take an appropriate point $P$ such that $\left(S(Q, P), W^{\triangle}(\boldsymbol{a})\right)$ is a mixed twistor. We denote the associated graded vector bundle by $\mathcal{G} r^{\triangle}(\boldsymbol{a})$.

Consider the morphism $N_{i}^{\triangle}: S(Q, P) \longrightarrow S(Q, P) \otimes \mathcal{O}_{\mathbb{P}^{1}}(2)$ for $i \in I$. Due to Lemma 2.10, we obtain the following morphisms for $h$ and $i \in I$ :

$$
\widetilde{N}_{i, h}^{\triangle}: \mathcal{G} r^{\triangle}(\boldsymbol{a})_{h} \longrightarrow \mathcal{G} r^{\triangle}(\boldsymbol{a})_{h-1} \otimes \mathcal{O}(2)
$$

Lemma 5.20. Consider the case $\lambda=0 \in \mathbb{P}^{1}$ and $Q$ as above. We have the following implication:

$$
N_{i \mid 0}^{\triangle}\left(W^{\triangle}(\boldsymbol{a})_{h \mid 0}\right) \subset W^{\triangle}(\boldsymbol{a})_{h-2 \mid 0} .
$$

Proof. We describe $\theta$ as

$$
\theta=\sum_{j=1}^{l} f_{j} \cdot \frac{d z_{j}}{z_{j}}+\sum_{j=l+1}^{n} g_{j} \cdot d z_{j} .
$$

We know that $\left|f_{i}\right|_{h} \leq C \cdot\left(-\log \left|z_{i}\right|\right)^{-1}$. Thus we have

$$
\left|\varphi^{*}\left(f_{i}\right)\right|_{\varphi^{*} h} \leq C^{\prime} \cdot(-\log |t|)^{-1} .
$$

Here $\varphi$ denotes the morphism given in (53) for $\boldsymbol{a}$. Consider the section $s$ of ${ }^{\diamond} \varphi^{*} E$ over $\Delta$. Let $\operatorname{deg}(s)$ be the degree of $s(O)$ with respect to the weight filtration of $\operatorname{Res}\left(\varphi^{*}(\theta)\right)$. Then we have the norm estimate:

$$
|s|_{\varphi^{*}(h)} \sim(-\log |t|)^{\operatorname{deg}(s) / 2} .
$$

It implies the following:

$$
\left|\varphi^{*}\left(f_{i}\right) \cdot s\right|_{\varphi^{*}(h)} \leq C^{\prime \prime} \cdot(-\log |t|)^{(\operatorname{deg}(s)-2) / 2} .
$$

By the norm estimate for the sections on the punctured disc, we can conclude that the degree of $\varphi^{*} f_{i} \cdot s(O)$ is less than $\operatorname{deg}(s)-2$. Thus we are done.

q.e.d.

We have the following immediate corollary.

Corollary 5.3. For $i \in I$, let $\widetilde{N}_{i, h}^{\triangle}$ be the morphism in (54). Then we have $\widetilde{N}_{i, h \mid 0}^{\triangle}=0$. We also have $\widetilde{N}_{i, h \mid \infty}^{\triangle}=0$. 
Proof. The first claim is obvious from Lemma 5.20. The second claim is obtained by applying Lemma 5.20 to the tame nilpotent harmonic bundle $\left(E^{\dagger}, \theta^{\dagger}, h\right)$.

q.e.d.

Corollary 5.4. Let $\widetilde{N}_{i, h}^{\triangle}$ be the morphism in (54) for $i \in I$. Then $\tilde{N}_{i, h}^{\triangle}$ is, in fact, 0 .

Proof. Since $\left(S(Q, P), W^{\triangle}\right)$ is a mixed twistor, the vector bundles $\mathcal{G} r_{h}(\boldsymbol{a})$ and $\mathcal{G} r_{h-1}(\boldsymbol{a}) \otimes \mathcal{O}_{\mathbb{P}^{1}}(2)$ are isomorphic to direct sums of $\mathcal{O}(h)$ and $\mathcal{O}(h+1)$ respectively. Thus $\widetilde{N}_{i, h}^{\triangle}$ is a section of the vector bundle isomorphic to a direct sum of $\mathcal{O}(1)$.

On the other hand, we know that $\widetilde{N}_{i, h}^{\triangle}$ vanishes at two points $\{0, \infty\}$ due to Corollary 5.4. Thus we can conclude that $\tilde{N}_{i, h}^{\triangle}$ vanishes over $\mathbb{P}^{1}$.

q.e.d.

As a direct corollary, we obtain the following important theorem.

Theorem 5.2. The morphism

$$
N_{i}^{\triangle}:\left(S(Q, P), W^{\triangle}(\boldsymbol{a})\right) \longrightarrow\left(S(Q, P), W^{\triangle}(\boldsymbol{a})\right) \otimes \mathcal{O}_{\mathbb{P}^{1}}(2) \quad(i \in I)
$$

gives morphisms of mixed twistor.

Proof. The claim is equivalent to $N_{i}^{\triangle} \cdot W^{\triangle}(\boldsymbol{a})_{h} \subset W^{\triangle}(\boldsymbol{a})_{h-2} \otimes$ $\mathcal{O}_{\mathbb{P}^{1}}(2)$. It is proved in Corollary 5.4.

\subsubsection{Some consequences}

Theorem 5.2 implies the following, for example.

Corollary 5.5. The conjugacy classes of $N_{i \mid \lambda}^{\triangle}$ are independent of a choice of $\lambda \in \mathbb{P}^{1}$ for each $i \in I$. Moreover the conjugacy classes of $N^{\triangle}(\boldsymbol{a})_{\mid \lambda}$ are independent of a choice of $\lambda \in \mathbb{P}^{1}$ for each $\boldsymbol{a} \in \mathbf{C}^{I}$.

Proof. We only have to use Lemma 2.21. q.e.d.

The claim of Corollary 5.5 for $\lambda \neq 0, \infty$ is rather obvious. However the fact that the conjugacy classes of $N_{j}^{\triangle}$ does not degenerate at $\lambda=$ $0, \infty$ is not trivial.

In each point $Q \in D_{i}$, the nilpotent map $N_{i \mid(\lambda, Q)}$ induces the weight filtration $W_{i \mid(\lambda, Q)}$.

Corollary 5.6. The conjugacy classes of $N_{i \mid(\lambda, Q)}$ are independent of $(\lambda, Q) \in \mathcal{D}_{i}$. As a result, the filtration $\left\{W_{i \mid(\lambda, Q)} \mid(\lambda, Q) \in \mathcal{D}_{i}\right\}$ forms the filtration of ${ }^{\diamond} \mathcal{E}_{\mathcal{D}_{i}}$ by vector subbundles. 
Proof. Fix $\lambda \neq 0$. Then it is easy to see that the conjugacy classes of $N_{i \mid(\lambda, Q)}$ are independent of a choice of $Q \in D_{i}$. To see it, we only have to use a normalizing frame, for example.

Fix $Q \in D_{i}$. Then we know that the conjugacy classes of $N_{i \mid(\lambda, Q)}$ are independent of $\lambda$ (Lemma 2.21). Thus we obtain our result. q.e.d.

For any subset $I \subset \underline{l}$, we put $\mathcal{D}_{I}=\bigcap_{i \in I} \mathcal{D}_{i}$. On $\mathcal{D}_{I}$, we have nilpotent maps, $N(\boldsymbol{a})=\sum_{i \in I} a_{i} \cdot N_{i \mid \mathcal{D}_{I}}$ for any $\boldsymbol{a} \in \mathbf{C}^{I}$.

Corollary 5.7. The weight filtrations $W(\boldsymbol{a})_{\mid(\lambda, Q)}$ of $N(\boldsymbol{a})_{\mid(\lambda, Q)}$ form the filtration of ${ }^{\diamond} \mathcal{E}_{\mid \mathcal{D}_{I}}$ by vector subbundles.

Proof. Similar to Corollary 5.6.

q.e.d.

On $D_{j}$, we have the residues $N_{i \mid \mathcal{D}_{j}}$ for $i \leq j$. We put $N(\underline{j})=$ $\sum_{i \leq j} N_{i \mid \mathcal{D}_{\underline{j}}}$. We have the weight filtration $W(\underline{j})$ of $N(\underline{j})$, which is a filtration of ${ }^{\diamond} \mathcal{E}_{\mathcal{D}_{\underline{j}}}$. In particular, we have the filtrations $W(\underline{j})$ on ${ }^{\diamond} \mathcal{E}_{\mathcal{D}_{\underline{m}}}$ for any $j \leq m$.

Lemma 5.21. Let $\boldsymbol{h}=\left(h_{1}, \ldots, h_{m}\right)$ be an m-tuple of integers. The intersections $\bigcap_{j=1}^{m} W(\underline{j})_{h_{j}}$ form a vector subbundle of ${ }^{\diamond} \mathcal{E}_{\mathcal{D}_{\underline{m}}}$.

Proof. Fix $\lambda \neq 0$. Then the rank of $\bigcap_{j=1}^{m} W(\underline{j})_{h_{j} \mid(\lambda, Q)}$ is easily seen to be independent of $Q \in D_{m}$. We only have to use a normalizing frame, for example.

Fix $Q \in D_{\underline{m}}$. Let $I$ denote the set $\left\{i \in \underline{l} \mid Q \in D_{i}\right\}$. Let $\boldsymbol{a} \in$ $\mathbf{Q}_{>0}^{I}$ be an element such that $N(\boldsymbol{a})_{\mid(\lambda, Q)}$ is general for any $\lambda$. We pick an appropriate point $P$ such that the vector bundle $S(Q, P)$ with the filtration $W^{\triangle}(\boldsymbol{a})$ is a mixed twistor. Then we know that $\bigcap_{j=1}^{m} W^{\triangle}(\underline{j})_{h_{j}}$ is a sub mixed twistor of $S(Q, P)$. In particular, we obtain that the rank of $\bigcap_{j=1}^{m} W(\underline{j})_{h_{j} \mid(\lambda, Q)}$ is independent of $\lambda \in \mathbf{C}_{\lambda}$ (Lemma 2.25). Thus we obtain our result.

q.e.d.

Let $\mathcal{G} r^{\triangle}(1)$ denote the associated graded vector bundle to $W^{\triangle}(\underline{1})$. We remark the following.

Lemma 5.22. We have $c_{1}\left(\mathcal{G} r_{h}^{\triangle(1)}\right)=h \cdot \operatorname{rank}\left(\mathcal{G} r_{h}^{\triangle(1)}\right)$. Here $c_{1}(\mathcal{F})$ denotes the first Chern class of a coherent sheaf $\mathcal{F}$ on $\mathbb{P}^{1}$.

Proof. If $Q \in D_{1}$ is contained in $D_{1}-\bigcup_{i=2}^{l} D_{1} \cap D_{i}$, then we only have to consider the restriction of the harmonic bundle to a curve which transversally intersects with $D_{1}$ at $Q$. In the general case, we use the topological invariance of the Chern class.

q.e.d. 


\subsubsection{The graded part}

Let $Q$ be a point of $D_{\underline{m}}$. We put $I=\left\{i \mid Q \in D_{i}\right\}$. Let $\boldsymbol{a}$ be an element of $\mathbf{Q}_{>0}^{I}$ such that $N^{\triangle}(\boldsymbol{a})$ is general. For any $j \leq m$, we put $N^{\triangle}(\underline{j}):=\sum_{i \leq j} N_{i}^{\triangle}$. Let $W^{\triangle}(\underline{j})$ denote the weight filtration of $N^{\triangle}(\underline{j})$. In particular, we obtain the associated graded vector bundle $\mathcal{G} r^{\triangle(1)}$ of $W^{\triangle}(\underline{1})$. When $\left(S(W, P), W^{\triangle}(\boldsymbol{a})\right)$ is a mixed twistor, we have the naturally induced mixed twistor structure on $\left(\mathcal{G} r^{\triangle(1)}, W^{\triangle(1)}(\boldsymbol{a})\right)$.

We have the induced morphisms $N^{\triangle(1)}(\underline{j}): \mathcal{G} r^{\triangle(1)} \longrightarrow \mathcal{G} r^{\triangle(1)} \otimes$ $\mathcal{O}_{\mathbb{P}^{1}}(2)$. They are again the morphisms of mixed twistors. In particular, the conjugacy classes of $N^{\triangle(1)}(\underline{j})_{\mid \lambda}$ are not independent of $\lambda \in \mathbb{P}^{1}$.

We will use the following special case later.

Lemma 5.23. The morphism $N^{\triangle(1)}(\underline{2})$ induces the filtration $W\left(N^{\triangle(1)}(\underline{2})\right)$ on $\mathcal{G} r^{\triangle(1)}=\bigoplus \mathcal{G} r_{h}^{\triangle(1)}$ by vector subbundles. Thus we obtain the graded vector bundle $\mathcal{G} r_{\left(h_{1}, h_{2}\right)}^{W\left(N^{\Delta(1)}(2)\right)}:=G r_{h_{2}}^{W\left(N^{\Delta(1)}(\underline{2})\right)}\left(\mathcal{G} r_{h_{1}}^{\triangle(1)}\right)$.

On $\mathcal{D}_{1}$, we obtain the graded vector bundle $\mathcal{G} r^{(1)}$ of $W(\underline{1})$. For $(\lambda, Q) \in \mathcal{D}_{\underline{m}}$, we have the induced filtrations $W^{(1)}(\underline{m})_{\mid(\lambda, Q)}$ on $\mathcal{G} r_{\mid(\lambda, Q)}^{(1)}$. We also obtain the morphism $N^{(1)}(\underline{m})_{\mid(\lambda, Q)} \in \operatorname{End}\left(\mathcal{G} r_{\mid(\lambda, Q)}^{(1)}\right)$.

\section{Lemma 5.24.}

- The filtrations $\left\{W^{(1)}(\underline{m})_{\mid(\lambda, Q)} \mid(\lambda, Q) \in \mathcal{D}_{\underline{m}}\right\}$ form a filtration of ${ }^{\diamond} \mathcal{E}_{\mid \mathcal{D}_{\underline{m}}}$ by vector subbundles.

- The conjugacy classes of $N^{(1)}(\underline{m})_{\mid(\lambda, Q)}$ are independent of a choice of $(\lambda, Q) \in \mathcal{D}_{\underline{m}}$.

Proof. Fix $\lambda \neq 0$. Then the claims can be checked by a normalizing frame.

Fix $Q \in D_{\underline{m}}$. Let $I$ denote the set $\left\{i \in \underline{l} \mid Q \in D_{i}\right\}$. Let $\boldsymbol{a} \in$ $\mathbf{Q}_{>0}^{I}$ be an element such that $N(\boldsymbol{a})_{\mid(\lambda, Q)}$ is general for any $\lambda$. We pick an appropriate point $P$ such that the vector bundle $S(Q, P)$ with the filtration $W^{\triangle}(\boldsymbol{a})$ is a mixed twistor.

Then we know that $W^{\triangle(1)}(\underline{m})$ is a sub mixed twistor of $\left(\mathcal{G} r^{\triangle(1)}\right.$, $\left.W^{\triangle}(\boldsymbol{a})\right)$. Thus the rank of $W^{(1)}(\underline{m})_{h}$ is independent of $\lambda$. Since $N^{\triangle(1)}(\underline{m})$ is a morphism of mixed twistor, the conjugacy classes are independent of a choice of $\lambda$. Thus we obtain the result. $\quad$ q.e.d. 
Lemma 5.25. The rank and the first Chern class of $\mathcal{G} r_{\left(h_{1}, h_{2}\right)}^{W\left(N^{\Delta(1)}(2)\right)}$ is independent of a choice of $Q$ and $P$.

Proof. We have already seen the independence of the rank. Since the dependence of the vector bundle on $Q$ and $P$ is continuous, the Chern class is invariant. q.e.d.

\subsubsection{The weak constantness of the filtrations}

We continue to use the notation in the previous subsubsections. By using Theorem 5.2, we can show the following weak constantness of the filtrations on the positive cones.

Proposition 5.5. Let $\boldsymbol{a}_{1}, \boldsymbol{a}_{2} \in \mathbf{C}^{I}$ be general. Then $W^{\triangle}\left(\boldsymbol{a}_{1}\right)=$ $W^{\triangle}\left(\boldsymbol{a}_{2}\right)$.

Proof. Let $\boldsymbol{a}_{1}$ be general. We have already known that $N^{\triangle}(\boldsymbol{a})$ drops the degree with respect to the filtration $W^{\triangle}\left(\boldsymbol{a}_{1}\right)$ by 2 , for any $\boldsymbol{a}$. For nonnegative $k \geq 0$, consider the following morphism:

$$
\left(N^{\triangle}(\boldsymbol{a})\right)^{k}: \mathcal{G} r_{k}^{\triangle} \longrightarrow \mathcal{G} r_{-k}^{\triangle} \otimes \mathcal{O}_{\mathbb{P}^{1}}(2 k) .
$$

It is isomorphic when $\boldsymbol{a}=\boldsymbol{a}_{1}$. Thus there is a Zariski open subset $U_{k}$ of $\mathbf{C}^{n}$ such that $\left(N^{\triangle}(\boldsymbol{a})\right)^{k}$ is isomorphic for any $\boldsymbol{a} \in O_{k}$. Then we know that there is a Zariski open subset $U$, such that $W^{\triangle}(\boldsymbol{a})=W^{\triangle}\left(\boldsymbol{a}_{1}\right)$ for any $\boldsymbol{a} \in U$. Then we obtain $W^{\triangle}(\boldsymbol{a})=W^{\triangle}\left(\boldsymbol{a}_{1}\right)$ if $\boldsymbol{a}$ is general. q.e.d.

\section{Limiting harmonic bundle in one direction}

\subsection{The method of comparison}

We put $X=\Delta^{n}=\left\{\left(\zeta_{1}, \ldots, \zeta_{n}\right) \in \Delta^{n}\right\}, D_{i}:=\left\{\zeta_{i}=0\right\}$, and $D=$ $\bigcup_{i=1}^{l} D_{i}$ for $l \leq n$. Let $\left(E, \bar{\partial}_{E}, \theta, h\right)$ be a tame nilpotent harmonic bundle with trivial parabolic structure over $X-D$. We have the deformed holomorphic bundle $(\mathcal{E}, \mathbb{D}, h)$ on $\mathcal{X}-\mathcal{D}$, and the prolongment ${ }^{\diamond} \mathcal{E}$. We have the residue $N_{i}:=\operatorname{Res}_{\mathcal{D}_{i}}(\mathbb{D})$.

We have already known that the conjugacy classes of $N_{1 \mid(\lambda, Q)}$ are independent of a choice of $(\lambda, Q) \in \mathcal{D}_{1}$ (Corollary 5.6). We have the weight filtration $W(\underline{1})$ of $N_{1}$. For any $k \geq 0$ and $h \in \mathbb{Z}$, we have the number $d(k, h):=\operatorname{dim} P_{k} G r_{h}^{(1)}$ determined by the conjugacy class of $N_{1}$. 
Take a holomorphic frame $\boldsymbol{v}$ of ${ }^{\diamond} \mathcal{E}$ over $\mathcal{X}$ satisfying the following:

- $\boldsymbol{v}=\left(v_{k, h, \eta} \mid k \geq 0, h \in \mathbb{Z}, \eta=1, \ldots, d(k, h)\right)$.

- $N_{1}\left(v_{k, h, \eta}\right)=v_{k, h-2, \eta}$ if $h>-k$, and $N_{1}\left(v_{k,-k, \eta}\right)=0$.

Note that $N_{1}$ is represented by a constant matrix with respect to the frame $\boldsymbol{v}_{\mid \mathcal{D}_{1}}$.

We put $\tilde{X}:=\Delta^{n}=\left\{\left(z_{1}, \ldots, z_{n}\right) \in \Delta^{n}\right\}, \widetilde{D}_{i}=\left\{z_{i}=0\right\}$, and $\widetilde{D}=\bigcup_{i=1}^{l} \widetilde{D}_{i}$. We have the morphism $\widetilde{X} \longrightarrow X$ defined as follows:

$$
\phi^{*}\left(\zeta_{i}\right)= \begin{cases}\prod_{j=i}^{l} z_{j}, & (i \leq l) \\ z_{i}, & (i>l) .\end{cases}
$$

We obtain the tame nilpotent harmonic bundle $\phi^{*}\left(E, \bar{\partial}_{E}, \theta, h\right)$, and the deformed holomorphic bundle $\phi^{*}(\mathcal{E}, \mathbb{D}, h)$. We put $\widetilde{\mathcal{E}}:=\phi^{*} \mathcal{E}$. We also have the prolongment ${ }^{\diamond} \widetilde{\mathcal{E}}=\phi^{* \diamond} \mathcal{E}$.

We have the projection $\mathfrak{q}_{1}: \Omega_{X}^{1,0} \longrightarrow q_{1}^{*} \Omega_{\Delta}^{1,0}$, and $\mathfrak{q}_{1}: \Omega_{X}^{1,0}(\log D) \longrightarrow$ $q_{1}^{*} \Omega_{\Delta}^{1,0}(\log O)$ :

$$
\mathfrak{q}_{1}\left(\sum_{i=1}^{n} f_{i} \cdot d z_{i}\right):=f_{1} \cdot d z_{1} .
$$

From the $\lambda$-connection

$$
\widetilde{\mathbb{D}}=\phi^{*} \mathbb{D}: \Gamma\left(\widetilde{\mathcal{X}},{ }^{\diamond} \phi^{*} \mathcal{E}\right) \longrightarrow \Gamma\left(\widetilde{\mathcal{X}}, \diamond \phi^{*} \mathcal{E} \otimes p_{\lambda}^{*} \Omega_{X}^{1,0}(\log D)\right),
$$

we obtain the family of $\lambda$-connections along the $z_{1}$-direction:

$$
\mathfrak{q}_{1}(\widetilde{\mathbb{D}}): \Gamma(\widetilde{\mathcal{X}}, \diamond \widetilde{\mathcal{E}}) \longrightarrow \Gamma\left(\widetilde{\mathcal{X}},{ }^{\diamond} \widetilde{\mathcal{E}} \otimes p_{\lambda}^{*} q_{1}^{*} \Omega_{\Delta}(\log O)\right)
$$

The residue $\operatorname{Res}_{\widetilde{\mathcal{D}}_{1}}\left(\mathfrak{q}_{1}(\widetilde{\mathbb{D}})\right)$ is same as $\phi^{*} N_{1}$.

We have the holomorphic frame $\widetilde{\boldsymbol{v}}:=\phi^{*} \boldsymbol{v}$. We have the $\lambda$-connection form $A \in \Gamma\left(\widetilde{\mathcal{X}}, M(r) \otimes \mathcal{O}_{\mathcal{X}}\right)$ of $\widetilde{\mathbb{D}}$ with respect to $\widetilde{\boldsymbol{v}}$, that is, $\widetilde{\mathbb{D}} \widetilde{\boldsymbol{v}}=$ $\widetilde{\boldsymbol{v}} \cdot A \cdot d z_{1} / z_{1}$.

Lemma 6.1. The restrictions $A_{\mid \widetilde{\mathcal{D}}_{i}}(i=1, \ldots, l)$ are constant, say $A_{0}$. 
Proof. Clear from our construction. Note that $\phi\left(\widetilde{\mathcal{D}}_{i}\right) \subset \mathcal{D}_{1}$. q.e.d.

Let $V$ be ${ }^{\diamond} \mathcal{E}_{\mid(0, O)}$ and $N$ be the residue $\operatorname{Res}(\mathbb{D})_{\mid(0, O)}$. From the pair $(V, N)$, we have a model bundle $E(V, N)=\left(E_{0}, \theta_{0}, h_{0}\right)$ on $\Delta^{*}$. We denote the deformed holomorphic bundle by $\left(\mathcal{E}_{0}, \mathbb{D}_{0}, h_{0}\right)$. We have the canonical frame $\boldsymbol{v}_{0}$ such that $\mathbb{D}_{0} \boldsymbol{v}_{0}=\boldsymbol{v}_{0} \cdot A_{0} \cdot d z / z$.

Let $q_{1}$ denote the projection $\Delta^{n} \longrightarrow \Delta$ onto the first component. We put $\widetilde{\mathcal{E}}_{0}:=q_{1}^{*} \mathcal{E}_{0}$. We have the $\lambda$-connection $\widetilde{\mathbb{D}}_{0}:=q_{1}^{*} \mathbb{D}_{0}$ along the $z_{1}$-direction. We also put $\widetilde{\boldsymbol{v}}_{0}:=q_{1}^{*} \boldsymbol{v}_{0}$.

Due to the frames $\widetilde{\boldsymbol{v}}$ and $\widetilde{\boldsymbol{v}}_{0}$, we obtain the holomorphic isomorphism $\Phi: \diamond \widetilde{\mathcal{E}}_{0} \longrightarrow{ }^{\diamond} \widetilde{\mathcal{E}}$.

Lemma 6.2. Note the following:

- We have $\Phi \circ \widetilde{\mathbb{D}}_{0}-\widetilde{\mathbb{D}} \circ \Phi=0$ on $\widetilde{\mathcal{D}}_{i}$ for $i=2, \ldots$, . Similarly we have $\Phi^{-1} \circ \widetilde{\mathbb{D}}-\widetilde{\mathbb{D}}_{0} \circ \Phi^{-1}=0$ on $\widetilde{\mathcal{D}}_{i}$ for $i=2, \ldots, l$.

- We have $\operatorname{Res}\left(\Phi \circ \widetilde{\mathbb{D}}_{0}-\widetilde{\mathbb{D}} \circ \Phi\right)=0$ on $\widetilde{\mathcal{D}}_{1}$. Similarly we have $\operatorname{Res}\left(\Phi^{-1} \circ \widetilde{\mathbb{D}}-\widetilde{\mathbb{D}}_{0} \circ \Phi^{-1}\right)=0$ on $\widetilde{\mathcal{D}}_{1}$.

Proof. Clear from our construction. q.e.d.

We have holomorphic bundles $\operatorname{Hom}\left({ }^{\diamond} \widetilde{\mathcal{E}}_{0}, \diamond \widetilde{\mathcal{E}}\right)$ and $\operatorname{Hom}\left({ }^{\diamond} \widetilde{\mathcal{E}},{ }^{\diamond} \widetilde{\mathcal{E}}_{0}\right)$. We have the naturally defined family of the $\lambda$-connections along the $z_{1}$ direction. induced by $\mathfrak{q}_{1}(\widetilde{\mathbb{D}})$ and $\widetilde{\mathbb{D}}_{0}$. We denote them by $\mathbb{D}_{1}$ and $\mathbb{D}_{2}$.

The morphism $\Phi$ and $\Phi^{-1}$ can be regarded as the sections of $\operatorname{Hom}\left(\diamond \widetilde{\mathcal{E}}_{0}, \diamond \widetilde{\mathcal{E}}\right)$ and $\operatorname{Hom}\left(\diamond \widetilde{\mathcal{E}}, \diamond \widetilde{\mathcal{E}}_{0}\right)$ respectively. Then Lemma 6.2 can be reworded as follows:

\section{Lemma 6.3.}

- $\mathbb{D}_{1} \Phi$ is holomorphic section of $\operatorname{Hom}\left({ }^{\diamond} \widetilde{\mathcal{E}}_{0}, \diamond \widetilde{\mathcal{E}}\right) \otimes p_{\lambda}^{*} q_{1}^{*} \Omega_{\Delta}(\log O)$. It vanishes on $\bigcup_{i=2}^{l} \widetilde{\mathcal{D}}_{i}$.

- $\mathbb{D}_{2} \Phi$ is holomorphic section of $\operatorname{Hom}\left(\diamond \widetilde{\mathcal{E}}, \diamond \widetilde{\mathcal{E}}_{0}\right) \otimes p_{\lambda}^{*} q_{1}^{*} \Omega_{\Delta}(\log O)$. It vanishes on $\bigcup_{i=2}^{l} \widetilde{\mathcal{D}}_{i}$.

We explain our method to obtain some estimate of norms. We have the metric $q_{1}^{*}\left(h_{0}\right)$ of $\widetilde{\mathcal{E}}_{0}$. Let $a$ and $b$ be functions as follows:

- $a$ is a positive function defined over $\Delta^{* n-1}$. For simplicity, we assume that $a\left(z_{2}, \ldots, z_{n}\right)$ is a polynomial of $-\log \left|z_{2}\right|, \ldots,-\log \left|z_{n}\right|$.

- $b$ is a holomorphic function defined over $\Delta^{n-1}$, such that $\mid b\left(z_{2}\right.$, $\left.\ldots, z_{n}\right) \mid \leq 1$ for any $\left(z_{2}, \ldots, z_{n}\right) \in \Delta^{n-1}$. 
If we are given such functions $a$ and $b$, we put as follows:

$$
\widetilde{h}_{0}\left(\lambda, z_{1}, z_{2}, \ldots, z_{n}\right):=a\left(z_{2}, \ldots, z_{n}\right) \times h_{0}\left(\lambda, b\left(z_{2}, \ldots, z_{n}\right) \cdot z_{1}\right) .
$$

From the metrics $\widetilde{h}$ and $\widetilde{h}_{0}$, we obtain the metrics of $\operatorname{Hom}\left(\widetilde{\mathcal{E}}_{0}, \widetilde{\mathcal{E}}\right)$ and $\operatorname{Hom}\left(\widetilde{\mathcal{E}}, \widetilde{\mathcal{E}}_{0}\right)$. We denote them by $|\cdot|_{\tilde{h}, \widetilde{h}_{0}}$.

Lemma 6.4. Let $C$ be a real number such that $0<C<1$. Let $R$ be a positive number.

- Assume that $\Phi$ is bounded with respect to the metrics $\widetilde{h}$ and $\widetilde{h}_{0}$ on the boundary:

$$
\Delta_{\lambda}(R) \times\left\{\left(z_{1}, \ldots, z_{n}\right) \in \widetilde{X}-\widetilde{D}|| z_{1} \mid=C\right\} .
$$

Then $\Phi$ is bounded over the following region:

$$
\Delta_{\lambda}(R) \times\left\{\left(z_{1}, \ldots, z_{n}\right) \in \widetilde{X}-\widetilde{D}|| z_{1} \mid \leq C\right\} .
$$

- Assume that $\Phi^{-1}$ is bounded with respect to the metrics $\widetilde{h}_{0}$ and $\widetilde{h}_{0}$ on the boundary $\Delta_{\lambda}(R) \times\left\{\left(z_{1}, \ldots, z_{n}\right) \in \widetilde{X}-\widetilde{D},|| z_{1} \mid=C\right\}$. Then $\Phi^{-1}$ is bounded over $\Delta_{\lambda}(R) \times\left\{\left(z_{1}, \ldots, z_{n}\right) \in \widetilde{X}-\widetilde{D}|| z_{1} \mid \leq C\right\}$.

Proof. Since $\mathbb{D}_{1} \Phi$ is holomorphic, and since $\mathbb{D}_{1} \Phi$ vanishes on $\bigcup_{i=1}^{l} \widetilde{\mathcal{D}}_{i}$, we obtain the inequality $\left|\mathbb{D}_{1} \Phi\right|_{\tilde{h}, \widetilde{h}_{0}}<C_{\epsilon} \cdot\left|z_{1}\right|^{-\epsilon}$ over $\Delta_{\lambda}(R) \times(\widetilde{X}-\widetilde{D})$ for any $0<\epsilon<1$. Then we can dominate the values $|\Phi|_{\tilde{h}, \tilde{h}_{0}}$ by the boundary values, due to the same argument as that in Proposition 4.7. Thus we obtain the result.

q.e.d.

Let us use the method. Let $M$ be an integer. We put as follows:

$$
\widetilde{h}_{M}\left(\lambda, z_{1}, \ldots, z_{n}\right):=\prod_{i=2}^{l}\left(-\log \left|z_{i}\right|\right)^{M} \cdot h_{0}\left(\lambda, z_{1}\right) .
$$

Lemma 6.5. If $M$ is sufficiently larger than 0 , then $\Phi$ is bounded. If $M$ is sufficiently smaller than 0 , then $\Phi^{-1}$ is bounded.

Proof. The claims are consequences of Lemma 6.4 and Lemma 4.15. Note that Lemma 4.15 is stated in the case $\lambda=1$. However the argument works for any $\lambda$.

q.e.d.

We reword the lemma as follows: Let $\boldsymbol{v}_{1}$ be a holomorphic frame of ${ }^{\diamond} \mathcal{E}$ over $\mathcal{X}$, compatible with the filtration $W(\underline{1})$ on $\mathcal{D}_{1}$. Then we obtain 
the frame $\boldsymbol{v}_{2}=\phi^{*} \boldsymbol{v}_{1}$ of $\diamond \widetilde{\mathcal{E}}$ over $\widetilde{\mathcal{X}}$. It is compatible with the filtration on $\widetilde{\mathcal{D}}_{1}$. We put $2 \cdot k\left(v_{2, i}\right):=\operatorname{deg} W(\underline{1})\left(v_{2, i}\right)$. We have the $C^{\infty}$-frame $\boldsymbol{v}_{2}^{\prime}$ of $\widetilde{\mathcal{E}}$ over $\widetilde{\mathcal{X}}-\widetilde{\mathcal{D}}_{1}$, given by $v_{2 i}^{\prime}:=\left(-\log \left|z_{1}\right|\right)^{-k\left(v_{2 i}\right)} \cdot v_{2 i}$.

Corollary 6.1. Let $\epsilon$ and $R$ be any positive numbers. Consider the following region:

$$
\Delta_{\lambda}(R) \times\left\{\left(z_{1}, \ldots, z_{n}\right) \in \widetilde{X}-\widetilde{D}|\epsilon<| z_{j} \mid,(j=2, \ldots, l)\right\} .
$$

On the region, the frame $\boldsymbol{v}_{2}^{\prime}$ is adapted.

We also have the following estimate over $\Delta_{\lambda}(R) \times(\widetilde{X}-\widetilde{D})$ :

$$
C_{1} \prod_{i=2}^{l}\left(-\log \left|z_{i}\right|\right)^{-M} \leq\left|v_{2 i}\right|_{\tilde{h}} \cdot\left(-\log \left|z_{1}\right|\right)^{-k\left(v_{2 i}\right)} \leq C_{2} \prod_{i=2}^{l}\left(-\log \left|z_{i}\right|\right)^{M} .
$$

Here $C_{1}$ and $C_{2}$ denote some positive constant, and $M$ denotes a sufficiently large number.

\subsection{Taking limit}

\subsubsection{Replacement of notation}

We essentially use the setting in Subsection 6.1. For simplicity of the notation, we replace $\widetilde{X}$ with $X$, and make the same replacement for others. More precisely, we consider as follows:

We put $X=\Delta^{n}, D_{i}=\left\{z_{i}=0\right\}$ and $D=\bigcup_{i=1}^{l} D_{i}$ for $l \leq n$. Let $\left(E, \bar{\partial}_{E}, \theta, h\right)$ be a tame nilpotent harmonic bundle with trivial parabolic structure over $X-D$. As usual, $(\mathcal{E}, \mathbb{D})$ denote the deformed holomorphic bundle with the $\lambda$-connection.

Let $\phi: \Delta^{n} \longrightarrow \Delta^{n}$ be the morphism considered in Subsection 6.1. It gives a morphism $\phi: X-D \longrightarrow X-D$.

Assumption 6.1. Assume the following:

- We have a tame nilpotent harmonic bundle $\left(E_{1}, \theta_{1}, h_{1}\right)$ with trivial parabolic structure over $X-D$, and $(E, \theta, h)$ is $\phi^{*}\left(E_{1}, \theta_{1}, h_{1}\right)$.

Let $\left(\mathcal{E}_{1}, \mathbb{D}_{1}\right)$ be the deformed holomorphic bundle with $\lambda$-connection of $\left(E_{1}, \theta_{1}, h_{1}\right)$. On $\mathcal{D}_{1}$, we have the weight filtration $W(\underline{1})$ of $\diamond \mathcal{E}_{1}$, induced by the residue $\operatorname{Res}_{\mathcal{D}_{1}}\left(\mathbb{D}_{1}\right)$. Let $\boldsymbol{v}_{1}$ be a holomorphic frame of the prolongment ${ }^{\diamond} \mathcal{E}_{1}$ compatible with the filtration $W(\underline{1})$.

Then we have the holomorphic frame $\boldsymbol{v}=\phi^{*} \boldsymbol{v}_{1}$ of $\diamond \mathcal{E}$ over $\mathcal{X}$. It is compatible with the weight filtration $W(\underline{1})$ induced by $\operatorname{Res}_{\mathcal{D}_{1}}(\mathbb{D})$, which is same as the pull back of the filtration above. 
We put $2 \cdot k\left(v_{i}\right):=\operatorname{deg}^{W(\underline{1})}\left(v_{i}\right)$. We have the $C^{\infty}$-frame $\boldsymbol{v}^{\prime}$ of $\mathcal{E}$ over $\mathcal{X}-\mathcal{D}_{1}$, given by $v_{i}^{\prime}:=\left(-\log \left|z_{1}\right|\right)^{-k\left(v_{i}\right)} \cdot v_{i}$.

The following lemma is completely same as Corollary 6.1.

Lemma 6.6. Let $\epsilon$ and $R$ be any positive numbers. Consider the following region:

$$
\left\{\left(\lambda, z_{1}, \ldots, z_{n}\right) \in \mathcal{X}-\mathcal{D}|| \lambda|<R, \epsilon<| z_{i} \mid,(i=2, \ldots, l)\right\} .
$$

On the region, the frame $\boldsymbol{v}^{\prime}$ is adapted.

We also have the following estimate over the region $\Delta_{\lambda}(R) \times(X-D)$ :

$$
C_{1} \prod_{i=2}^{l}\left(-\log \left|z_{i}\right|\right)^{-M} \leq\left|v_{i}\right|_{h} \cdot\left(-\log \left|z_{1}\right|\right)^{-k\left(v_{i}\right)} \leq C_{2} \prod_{i=2}^{l}\left(-\log \left|z_{i}\right|\right)^{M} .
$$

Here $C_{1}$ and $C_{2}$ denote some positive constant, and $M$ denotes a sufficiently large number.

By the frame $\boldsymbol{v}$, we decompose ${ }^{\diamond} \mathcal{E}$ as follows:

$$
{ }^{\diamond} \mathcal{E}=\bigoplus_{h} U_{h}, \quad U_{h}:=\left\langle v_{i} \mid \operatorname{deg}^{W(1)}\left(v_{i}\right)=h\right\rangle .
$$

\subsubsection{Pull backs}

Let $m$ be a nonnegative integers. We have the morphism $\psi_{m, \underline{1}}: X \longrightarrow$ $X$ or $X-D \longrightarrow X-D$, defined as follows:

$$
\psi_{m, \underline{1}}\left(z_{1}, z_{2}, \ldots, z_{n}\right):=\left(z_{1}^{m}, z_{2}, \ldots, z_{n}\right) .
$$

Then we obtain the harmonic bundles $\psi_{m, \underline{1}}^{*}\left(E, \bar{\partial}_{E}, \theta, h\right)$ on $X-D$. We also obtain the deformed holomorphic bundles and the $\lambda$-connections $\left(\psi_{m, \underline{1}}^{*} \diamond \mathcal{E}, \psi_{m, \underline{1}}^{*} \mathbb{D}\right)$. We obtain the holomorphic frame $\boldsymbol{v}^{(m)}$ of $\psi_{m, \underline{1}}^{*} \mathcal{E}$, defined as follows:

$$
v_{i}^{(m)}:=\psi_{m, \underline{1}}^{-1}\left(v_{i}\right) \cdot m^{-k\left(v_{i}\right)} .
$$

We put $H^{(m)}:=H\left(\psi_{m, \underline{1}}^{*}(h), \boldsymbol{v}^{(m)}\right)$, which is an $\mathcal{H}(r)$-valued function.

Lemma 6.7. On any compact subset $K \subset X-D$, the $\mathcal{H}(r)$-valued functions $\left\{H^{(m)}\right\}$ and $\left\{H^{(m)-1}\right\}$ are bounded independently of $m$.

Proof. We put $H^{\prime}:=H\left(h, \boldsymbol{v}^{\prime}\right)$. Let $K^{\prime}$ be a compact subset of $\Delta^{* l-1} \times \Delta^{n-l}$. Then $\Delta_{1}^{*} \times K^{\prime}$ naturally gives a subset of $X-D$. We 
have $\psi_{m, \underline{1}}\left(\Delta_{1}^{*} \times K\right) \subset \Delta_{1}^{*} \times K$. Due to Lemma $6.6, H^{\prime}$ and $H^{\prime-1}$ are bounded over the region $\Delta_{1}^{*} \times K \subset X$.

We put as follows:

$$
\boldsymbol{v}^{\prime(m)}=\psi_{m, \underline{1}}^{*}\left(\boldsymbol{v}^{\prime}\right), \quad H^{\prime(m)}:=H\left(\psi_{m, \underline{1}}^{*}(h), \boldsymbol{v}^{\prime m}\right)=\psi_{m, \underline{1}}^{*} H^{\prime} .
$$

Then $H^{\prime(m)}$ and $H^{\prime(m)-1}$ are bounded over the region $\Delta_{1}^{*} \times K \subset X$, independently of $m$.

Let $L$ denote the diagonal matrix whose $(i, i)$-component is $\left(-\log \left|z_{1}\right|\right)^{k\left(v_{i}\right)}$. It is easy to check the following relation:

$$
L \cdot H^{\prime(m)} \cdot L=H^{(m)} .
$$

Thus we obtain our result.

q.e.d.

We have the $\lambda$-connection form $\mathcal{A} \in \Gamma\left(\mathcal{X}, M(r) \otimes p_{\lambda}^{*} \Omega_{X}^{1,0}(\log X)\right)$ of $\mathbb{D}$ with respect to the frame $\boldsymbol{v}$. We decompose $\mathcal{A}$ as follows:

$$
\mathcal{A}=\sum_{j} \mathcal{A}_{j}, \quad \mathcal{A}_{j} \in \Gamma\left(\mathcal{X}, M(r) \otimes p_{\lambda}^{*} q_{i}^{*} \Omega_{\Delta}(\log O)\right) .
$$

We obtain $A_{j} \in M(r) \otimes \mathcal{O}_{\mathcal{X}}$ satisfying the following:

$$
\mathcal{A}_{j}= \begin{cases}A_{1} \cdot \frac{d z_{1}}{z_{1}}, & (j=1), \\ A_{j} \cdot d z_{j}, & (j \neq 1) .\end{cases}
$$

Let $f_{A_{j}}$ denote the section of $\operatorname{End}\left({ }^{\diamond} \mathcal{E}\right)$ over $\mathcal{X}$, determined by the frame $\boldsymbol{v}$ and $A_{j}$, that is $f_{A_{j}}(\boldsymbol{v})=\boldsymbol{v} \cdot A_{j}$. The decomposition ${ }^{\diamond} \mathcal{E}=\bigoplus U_{h}$ induce the decomposition of $f_{j}$ as follows:

$$
f_{A_{j}}=\sum_{h, k} f_{A_{j}(h, k)}, \quad f_{A_{j}(h, k)}\left(U_{k}\right) \subset U_{h} .
$$

The section $f_{A_{j}(h, k)}$ induces the section of $M(r) \otimes \mathcal{O}_{\mathcal{X}}$ by the relation $f_{A_{j}(h, k)}(\boldsymbol{v})=\boldsymbol{v} \cdot A_{j(h, k)}$. Thus we obtain the decomposition $\mathcal{A}_{j}=$ $\sum_{h, k} A_{j(h, k)} \cdot d z_{j}$.

Lemma 6.8. We have the following vanishing results:

- If $h>k-2$, we have $A_{1(h, k) \mid \mathcal{D}_{1}}=0$.

- If $h>k$, we have $A_{j(h, k) \mid \mathcal{D}_{1}}=0$ for $j=2, \ldots, n$. 
Proof. Since $\mathcal{A}$ is the flat $\lambda$-connection form, we obtain the following equality:

$$
\lambda \cdot d \mathcal{A}+\mathcal{A} \wedge \mathcal{A}=0
$$

Note that $A_{1 \mid \mathcal{D}_{1}}$ is constant, by our construction, i.e., $d\left(A_{1 \mid \mathcal{D}_{1}}\right)=0$. It implies that $\left[A_{1 \mid \mathcal{D}_{1}}, A_{j \mid \mathcal{D}_{1}}\right]=0$ for any $j$, in other words, $f_{A_{1} \mid \mathcal{D}_{1}}$ and $f_{A_{j} \mid \mathcal{D}_{1}}$ are commutative. Hence the sections $f_{A_{j} \mid \mathcal{D}_{1}}$ preserves the filtration $W(\underline{1})$ on $\mathcal{D}_{1}$. Thus we obtain the second claim. Moreover $f_{A_{1} \mid \mathcal{D}_{1}}$ drops the degree by 2 . Thus we obtain the first claim. q.e.d.

We have the $\lambda$-connection form $\mathcal{A}^{(m)}$ of $\psi_{m, 1}^{*} \mathbb{D}$ with respect to the frame $\boldsymbol{v}^{(m)}$. We decompose $\mathcal{A}^{(m)}$ into $\sum_{j} \mathcal{A}_{j}^{(m)}$ as in the case of $\mathcal{A}$.

Lemma 6.9. We have the following equalities:

$$
\mathcal{A}_{j}^{(m)}= \begin{cases}\sum_{h, k} \psi_{m, \underline{1}}^{*} A_{1(h, k)} \cdot m^{(h-k+2) / 2} \cdot \frac{d z_{1}}{z_{1}} & (j=1) \\ \sum_{h, k} \psi_{m, \underline{1}}^{*} A_{j(h, k)} \cdot m^{(h-k) / 2} \cdot d z_{j} & (j \neq 1) .\end{cases}
$$

In particular, the sequences $\left\{\mathcal{A}^{(m)}\right\}$ converges to $\mathcal{A}^{(\infty)}=\sum_{j} \mathcal{A}_{j}^{(\infty)}$ given as follows:

$$
\mathcal{A}_{j}^{(\infty)}= \begin{cases}\sum_{h} \pi_{1}^{*} A_{1(h, h+2)} \cdot \frac{d z_{1}}{z_{1}} & (j=1) \\ \sum_{h} \pi_{1}^{*} A_{j(h, h)} \cdot d z_{j} & (j \neq 1) .\end{cases}
$$

Here $\pi_{1}$ denote the projection $\mathcal{X} \longrightarrow \mathcal{D}_{1}$, omitting $z_{1}$.

Proof. The $\lambda$-connection form of $\psi_{m, 1}^{*} \mathbb{D}^{*}$ with respect to the frame $\psi_{m, \underline{1}}^{*} \boldsymbol{v}$ is represented by $\psi_{m, \underline{1}}^{*} \mathcal{A}$. Thus we obtain our result by a direct calculation.

q.e.d.

We decompose $\theta$ into $\sum_{i=1}^{l} f_{i} \cdot d z_{i} / z_{i}+\sum_{i=l+1}^{n} g_{i} \cdot d z_{i}$. Then we have $\psi_{m, \underline{1}}^{*} \theta=m \cdot \psi_{m, \underline{1}}^{*}\left(f_{1}\right) \cdot d z_{1} / z_{1}+\sum_{i=2}^{l} \psi_{m, \underline{1}}^{*}\left(f_{i}\right) \cdot d z_{i} / z_{i}+\sum_{i=l+1}^{n} \psi_{m, \underline{1}}^{*}\left(g_{i}\right)$. $d z_{i}$.

Lemma 6.10. We have the following inequalities independent of $m$ :

$$
\begin{aligned}
&\left|m \cdot \psi_{m, \underline{1}}^{*}\left(f_{1}\right)\right|_{\psi_{m, \underline{1}}^{*}(h)} \leq C \cdot\left(-\log \left|z_{1}\right|\right)^{-1}, \\
&\left|\psi_{m, \underline{1}}^{*}\left(f_{i}\right)\right|_{\psi_{m, \underline{1}}^{*}(h)} \leq C \cdot\left(-\log \left|z_{i}\right|\right)^{-1},(2 \leq i \leq l), \\
&\left|\psi_{m, \underline{1}}^{*}\left(g_{i}\right)\right|_{\psi_{m, \underline{1}}^{*}(h)} \leq C,(l+1 \leq i \leq n) .
\end{aligned}
$$


Proof. We have the equality

$$
\psi_{m, \underline{1}}^{*}\left(\left(-\log \left|z_{1}\right|\right)^{-1}\right)=m^{-1}\left(-\log \left|z_{1}\right|\right)^{-1} .
$$

Thus we obtain the result due to Proposition 4.1

q.e.d.

In all we obtain the following.

Lemma 6.11. The sequence $\left\{\boldsymbol{v}^{(m)}\right\}$ satisfies Condition 3.2.

\subsubsection{Limit}

We can apply the result in Subsubsection 3.3.4. Let $F=\bigoplus_{i=1}^{r} \mathcal{O}_{X-D} \cdot u_{i}$ be a holomorphic bundle with the frame $\boldsymbol{u}=\left(u_{i}\right)$, over $X-D$. We put $\boldsymbol{e}^{(m)}:=\boldsymbol{v}_{\mid \mathcal{X}^{0}}^{(m)}$. It is the frame of ${ }^{\diamond} \psi_{m, \underline{1}}^{*} \mathcal{E}^{0}$ over $\mathcal{X}^{0}=\{0\} \times X$. The frames $\boldsymbol{e}^{(m)}$ and $\boldsymbol{u}$ give the holomorphic isomorphism $\Phi_{m}: \mathcal{E}^{0} \longrightarrow F$ over $X-D$.

The morphism $\Phi_{m}$ induces the structure of harmonic bundle on $F$. Namely, we obtain the metric $h^{(m)}$, the holomorphic Higgs fields $\left\{\theta^{(m)}\right\}$ defined over $X-D$, which are the image of $\psi_{m, \underline{1}}^{*}(h)$ and $\psi_{m, \underline{1}}^{*} \theta$ via the morphism $\Phi_{m}$. The tuple $\left(F, \bar{\partial}_{F}, \theta^{(m)}, h^{(m)}\right)$ gives a harmonic bundle for each $m$.

We obtain the deformed holomorphic bundles with $\lambda$-connection $\left(\mathcal{F}^{(m)}, \mathbb{D}^{(m)}\right)$. The morphism $\Phi_{m}$ induces the holomorphic isomorphism $\psi_{m, 1}^{*} \mathcal{E} \longrightarrow \mathcal{F}^{(m)}$. Thus we obtain the frame $\psi_{m, \underline{1}}\left(\boldsymbol{v}^{(m)}\right)$ of $\mathcal{F}^{(m)}$ over $\mathcal{X}-\mathcal{D}$.

Lemma 6.12. We can pick a subsequence $\left\{m_{i}\right\}$ of $\{m\}$ satisfying the following:

- We have the holomorphic Higgs field $\theta^{(\infty)}$ and the metric $h^{(\infty)}$. The sequence $\left\{\theta^{\left(m_{i}\right)}\right\}$ and $\left\{h^{\left(m_{i}\right)}\right\}$ converges to $\theta^{(\infty)}$ and $h^{(\infty)}$ respectively, on any compact subset $K \subset X-D$. We denote the deformed holomorphic bundle of $\left(F, \theta^{(\infty)}, h^{(\infty)}\right)$ by $\left(\mathcal{F}^{(\infty)}, \mathbb{D}^{(\infty)}\right)$.

- We have the holomorphic frame of $\boldsymbol{v}^{(\infty)}$ of $\mathcal{F}^{(\infty)}$ over $\mathcal{X}-\mathcal{D}$. The sequence $\left\{\Phi_{m}\left(\boldsymbol{v}^{\left(m_{i}\right)}\right)\right\}$ converges on any compact subset $K \subset$ $\mathcal{X}-\mathcal{D}$.

Proof. We only have to apply Proposition 3.2 and Lemma 3.30. 
Lemma 6.13. Let $N$ be a sufficiently large number. We have the following estimate over $\mathcal{X}-\mathcal{D}$, for the frame $\boldsymbol{v}^{(\infty)}=\left(v_{i}^{(\infty)}\right)$ :

$$
\left|v_{i}^{(\infty)}\right|_{h^{(\infty)}}<C \cdot\left(-\log \left|z_{1}\right|\right)^{k\left(v_{i}\right)} \prod_{j=2}^{l}\left(-\log \left|z_{j}\right|\right)^{N} .
$$

We also have the following estimate over $\mathcal{X}-\mathcal{D}$, for $\Omega\left(\boldsymbol{v}^{(\infty)}\right)=v_{1}^{(\infty)} \wedge$ $\cdots \wedge v_{r}^{(\infty)}$ :

$$
0<C_{1}<\left|\Omega\left(\boldsymbol{v}^{(\infty)}\right)\right|_{h^{(\infty)}}
$$

Proof. By our construction of $\boldsymbol{v}^{(m)}$, we obtain the estimate:

$$
\left|v_{i}^{(m)}\right|_{\psi_{m, \underline{1}}^{*}(h)}<C \cdot\left(-\log \left|z_{1}\right|\right)^{k\left(v_{i}\right)} \prod_{j=2}^{l}\left(-\log \left|z_{j}\right|\right)^{N} .
$$

Hence we obtain the inequality in the limit.

We have the inequality $0<C_{1}<|\Omega(\boldsymbol{v})|_{h}$. It induces the following:

$$
0<C_{1}<\left|\Omega\left(\boldsymbol{v}^{(m)}\right)\right|_{\psi_{m, \underline{1}}^{*}(h)} .
$$

Hence we obtain the inequality in the limit.

q.e.d.

Corollary 6.2. The frame $\boldsymbol{v}^{(\infty)}$ of $\mathcal{F}^{(\infty)}$ over $\mathcal{X}-\mathcal{D}$ naturally induces the frame of the prolongment ${ }^{\diamond} \mathcal{F}^{(\infty)}$ over $\mathcal{X}$.

The $\lambda$-connection form of $\mathbb{D}^{(\infty)}$ with respect to the frame $\boldsymbol{v}^{(\infty)}$ is given by $\mathcal{A}^{(\infty)}$.

\subsection{The decomposition in limit}

\subsubsection{Construction}

Let $\mathcal{U}_{h}$ denote the vector subbundle of ${ }^{\diamond} \mathcal{F}^{(\infty)}$ generated by

$$
\left\{v_{i}^{(\infty)} \mid \operatorname{deg}^{\mathcal{W}(\underline{1})}\left(v_{i}\right)=h\right\} .
$$

Lemma 6.14. The vector bundle $\mathcal{U}_{h}$ does not depend on a choice of the original frame $\boldsymbol{v}$ of ${ }^{\diamond} \mathcal{E}$ compatible with the filtration $W(\underline{1})$.

Proof. Let $\dot{\boldsymbol{v}}$ be another frame of $\diamond \mathcal{E}$ over $\mathcal{X}$ compatible with the filtration $W(\underline{1})$. We have the holomorphic functions $b_{j i}$ satisfying $v_{i}=$ 
$\sum_{j} b_{j} \dot{v}_{j}$. Since $\boldsymbol{v}$ and $\dot{\boldsymbol{v}}$ are compatible with the filtration $W(\underline{1})$, we have the following vanishing:

$$
b_{j i \mid \mathcal{D}_{1}}=0, \quad \text { if } \operatorname{deg}^{W(\underline{1})}\left(\dot{v}_{j}\right)>\operatorname{deg}^{W(\underline{1})}\left(v_{i}\right) .
$$

We obtain the relation of $\boldsymbol{v}^{(m)}$ and $\dot{\boldsymbol{v}}^{(m)}$ for each $m$ :

$$
v_{i}^{(m)}=\sum_{j} \psi_{m}^{*}\left(b_{j i}\right) \cdot m^{k\left(\dot{v}_{j}\right)-k\left(v_{i}\right)} \cdot \dot{v}_{j}^{(m)} .
$$

Here we have $2 k\left(\dot{v}_{j}\right)=\operatorname{deg}^{W(\underline{1})}\left(\dot{v}_{j}\right)$. Then we obtain the relation of $\boldsymbol{v}^{(\infty)}$ and $\dot{\boldsymbol{v}}^{(\infty)}$ as follows:

$$
v_{i}^{(\infty)}=\sum_{k\left(\dot{v}_{j}\right)=k\left(v_{i}\right)} \pi_{1}^{*}\left(b_{j i}\right) \cdot \dot{v}_{j}^{(\infty)} .
$$

Here $\pi_{1}$ denotes the projection $\mathcal{X} \longrightarrow \mathcal{D}_{1}$, omitting the first component. Thus $\mathcal{U}_{h}$ does not depend on a choice of the original frame. q.e.d.

Thus we obtain the decomposition $\mathcal{F}^{(\infty)}=\bigoplus_{h} \mathcal{U}_{h}$.

We have the $\lambda$-connection form

$$
\mathcal{A}^{(\infty)}=\sum_{j=1}^{l} A_{1}^{(\infty)} \cdot d z_{j} / z_{j}+\sum_{j=l+1}^{n} A_{j}^{(\infty)} \cdot d z_{j}
$$

of $\mathbb{D}^{(\infty)}$ with respect to the frame $\boldsymbol{v}^{(\infty)}$. Let $f_{A_{j}^{(\infty)}}$ denote the sections of $\operatorname{End}\left(\mathcal{F}^{(\infty)}\right)$ over $\mathcal{X}$ determined by $A_{j}^{(\infty)}$ and the frame $\boldsymbol{v}^{(\infty)}$.

\section{Lemma 6.15.}

- We have $f_{A_{1}^{(\infty)}}\left(\mathcal{U}_{h}\right) \subset \mathcal{U}_{h-2}$.

- When $j \neq 1$, the morphisms $f_{A_{j}^{(\infty)}}$ preserve the decomposition $\mathcal{F}^{(\infty)}=\bigoplus_{h} \mathcal{U}_{h}$

Proof. The claims immediately follow from Lemma 6.9 and Corollary 6.2 .

q.e.d.

\subsubsection{Orthogonality}

We put $\mathcal{U}_{h}^{\lambda}:=\mathcal{U}_{h \mid \mathcal{X}^{\lambda}}$.

Theorem 6.1. If $h \neq h^{\prime}$, then $\mathcal{U}_{h}^{0}$ and $\mathcal{U}_{h^{\prime}}^{0}$ are orthogonal. 
Proof. We only have to consider the case $X=\Delta$ and $D=\{O\}$. We use the notation $\psi_{m}$ instead of $\psi_{m, 1}$ for simplicity. We put $V=$ ${ }^{\diamond} \mathcal{E}^{0}{ }_{\mid(0, O)}$ and $N=\operatorname{Res}(\mathbb{D})_{\mid(0, O)}$. We have a model bundle $E(V, N)=$ $\left(E_{0}, \theta_{0}, h_{0}\right)$. Let $\left(\mathcal{E}_{0}, \mathbb{D}_{0}\right)$ denote the deformed holomorphic bundle with the $\lambda$-connection. We have the canonical frame $\boldsymbol{v}$ of the prolongment $\diamond^{\mathcal{E}_{0}}$, such that $\mathbb{D}_{0} \cdot \boldsymbol{v}=\boldsymbol{v} \cdot N \cdot d z / z$.

We put $\boldsymbol{e}_{0}:=\boldsymbol{v}_{0 \mid \mathcal{X}^{0}}$. Due to the frames $\boldsymbol{e}_{0}$ and $\boldsymbol{e}$, we obtain the holomorphic isomorphism $\mathcal{I}:{ }^{\diamond} \mathcal{E}_{0}^{0} \longrightarrow{ }^{\diamond} \mathcal{E}^{0} \operatorname{such}$ that $\operatorname{Res}\left(\mathcal{I} \circ \theta_{0}-\theta \circ \mathcal{I}\right)=$ 0 . We obtain $\psi_{m}^{*} \mathcal{I}: \psi_{m}^{*} \mathcal{E}_{0}^{0} \longrightarrow \psi_{m}^{*} \mathcal{E}^{0}$.

We take a limit of $\left(\mathcal{E}_{0}, \mathbb{D}_{0}, h_{0}\right)$ by using the frame $\boldsymbol{v}_{0}$, as in Subsection 6.2. Namely we have the frames $\boldsymbol{v}_{0}^{(m)}$ of $\psi_{m}^{*} \mathcal{E}_{0}$ defined as follows:

$$
v_{0 i}^{(m)}:=\psi_{m}^{-1}\left(v_{0 i}\right) \cdot m^{-k\left(v_{0 i}\right)} .
$$

We have the holomorphic isomorphism $\Phi_{0 m}: \mathcal{E}_{0}^{0} \longrightarrow F$ given by the frames $\boldsymbol{e}_{0}^{(m)}$ and $\boldsymbol{u}$. Note that we have $\Phi_{0 m}=\Phi_{m} \circ \psi_{m}^{*} \mathcal{I}$.

Then $\Phi_{0 m}$ induces the holomorphic Higgs field $\theta_{0}^{(m)}$ and the metric $h_{0}^{(m)}$ on $F$. The tuple $\left(F, \bar{\partial}_{F}, \theta_{0}^{(m)}, h_{0}^{(m)}\right)$ gives a harmonic bundle. We denote the deformed holomorphic bundles by $\left(\mathcal{F}_{0}^{(m)}, \mathbb{D}_{0}^{(m)}\right)$. We also obtain the holomorphic frames $\Phi_{0 m}\left(\boldsymbol{v}_{0}^{(m)}\right)$ of $\mathcal{F}_{0}^{(m)}$.

For the subsequence $\left\{m_{i}\right\}$, we have the limits $\theta_{0}^{(\infty)}, h_{0}^{(\infty)}$ and $\boldsymbol{v}_{0}^{(\infty)}$ of the sequences $\left\{\theta_{0}^{\left(m_{i}\right)}\right\},\left\{h_{0}^{\left(m_{i}\right)}\right\}$ and $\left\{\Phi_{0 m_{i}}\left(\boldsymbol{v}_{0}^{\left(m_{i}\right)}\right)\right\}$ respectively. By our construction, we have the equality $\theta_{0}^{(\infty)}=\theta^{(\infty)}$.

From the two harmonic bundles $\left(F, \theta^{(\infty)}, h^{(\infty)}\right)$ and $\left(F, \theta_{0}^{(\infty)}, h_{0}^{(\infty)}\right)$, we obtain the two deformed holomorphic bundles $\mathcal{F}^{(\infty)}$ and $\mathcal{F}_{0}^{(\infty)}$. Since the underlying $C^{\infty}$-vector bundles of them is same as $p_{\lambda}^{-1}(F)$, we have the natural $C^{\infty}$-isomorphism $\mathcal{I}^{(\infty)}: \mathcal{F}_{0}^{(\infty)} \longrightarrow \mathcal{F}^{(\infty)}$.

\section{Proposition 6.1.}

- The morphism $\mathcal{I}^{(\infty)}$ is holomorphic.

- $\mathcal{I}^{(\infty)}$ naturally induces the isomorphism of the prolongments ${ }^{\diamond} \mathcal{F}_{0}^{(\infty)}$ $\longrightarrow{ }^{\diamond} \mathcal{F}^{(\infty)}$.

- $\mathcal{I}^{(\infty)}$ preserves the weight filtrations of the residues $\operatorname{Res}\left(\mathbb{D}^{0}\right)$ and $\operatorname{Res}(\mathbb{D})$.

Proof. The holomorphic map $\mathcal{I}: \mathcal{E}_{0}^{0} \longrightarrow \mathcal{E}^{0}$ induces the $C^{\infty_{-}}$ isomorphism $\mathcal{I}: \mathcal{E}_{0} \longrightarrow \mathcal{E}$ defined over $\mathcal{X}-\mathcal{D}$. We have the elements 
$I_{i j}(\lambda, z) \in C^{\infty}(\mathcal{X}-\mathcal{D})$ determined as follows:

$\mathcal{I}\left(v_{0 j}\right)=\sum_{i} I_{i j} \cdot v_{i}, \quad$ or equivalently, $\quad \Phi_{00}\left(v_{0 j}^{(0)}\right)=\sum_{i} I_{i j} \cdot \Phi_{0}\left(v_{i}^{(0)}\right)$.

Similarly we obtain the functions $I_{i j}^{(m)} \in C^{\infty}(\mathcal{X}-\mathcal{D})$ determined as follows:

$$
\Phi_{0 m}\left(v_{0 j}^{(m)}\right)=\sum_{i} I_{i j}^{(m)} \cdot \Phi_{m}\left(v_{i}^{(m)}\right)
$$

Since $\left\{\Phi_{0 m_{i}}\left(\boldsymbol{v}_{0}^{\left(m_{i}\right)}\right)\right\}$ and $\left\{\Phi_{m_{i}}\left(\boldsymbol{v}^{\left(m_{i}\right)}\right)\right\}$ converge to $\boldsymbol{v}_{0}^{(\infty)}$ and $\boldsymbol{v}^{(\infty)}$ in $L_{k}^{p}$ for any large $k$ and for a sufficiently large $p$ over any compact subset $K \subset \mathcal{X}-\mathcal{D}$, the functions $\left\{I_{i j}^{\left(m_{i}\right)}\right\}$ converges similarly. We denote the limit by $I_{i j}^{(\infty)}$. We obtain the following relation:

$$
v_{0 j}^{(\infty)}=\sum_{i} I_{i j}^{(\infty)} \cdot v_{i}^{(\infty)}
$$

We will show that $I_{i j}^{(\infty)}$ are holomorphic, which implies that $\mathcal{I}^{(\infty)}$ is holomorphic. In fact, $I_{i j}^{(m)}$ are holomorphic along the direction of $\lambda$. Thus we will check that $I_{i j}^{(\infty)}$ are holomorphic along the direction of $z$.

We use the following lemma.

Lemma 6.16. Note that we have the equality $\left\|\psi_{m}^{-1}(f)\right\|_{Z, C}=$ $\|f\|_{Z, C^{n}}$ for any $C^{\infty}$-function $f$ on $\Delta^{*}$ such that $\|f\|_{Z, C}<\infty$.

Proof. By using the real coordinate $z=r \cdot \exp (\sqrt{-1} \alpha)$, we have the following:

$$
\begin{aligned}
\left\|\psi_{m}^{-1}(f)\right\|_{Z . C}: & =\int_{\Delta^{*}(C)}\left|\psi_{m}^{-1}(f)\right| \frac{d r \cdot d \alpha}{r \cdot(-\log r)} \\
& =\frac{1}{m} \int_{\Delta^{*}(C)} \psi_{m}^{-1}\left(|f| \frac{d r \cdot d \alpha}{r \cdot(-\log r)}\right) .
\end{aligned}
$$

Since the degree of the map $\psi_{m}: \Delta(C) \longrightarrow \Delta\left(C^{m}\right)$ is $m$, the right-hand side is same as the following:

$$
\int_{\Delta^{*}\left(C^{m}\right)}|f| \frac{d r \cdot d \alpha}{r \cdot(-\log r)}=:\|f\|_{Z, C^{m}} .
$$

Thus we are done.

q.e.d. 
Let us return to the proof of Proposition 6.1. Note the following equality:

$$
I_{i j}^{(m)}=\psi_{m}^{-1}\left(I_{i j}\right) \cdot m^{k\left(v_{i}\right)-k\left(v_{0 j}\right)} .
$$

Thus we obtain the following equality:

$$
\begin{aligned}
& \bar{z} \cdot \bar{\partial}_{z} I_{i j}^{(m)}(-\log |z|)^{k\left(v_{i}\right)-k\left(v_{0 j}\right)+1} \\
& =\psi_{m}^{-1}\left(\bar{z} \cdot \bar{\partial}_{z} I_{i j}(-\log |z|)^{k\left(v_{i}\right)-k\left(v_{0 j}\right)+1}\right) .
\end{aligned}
$$

Due to the result of Simpson, we know the finiteness for any $C<1$ :

$$
\|\left.\bar{z} \cdot \bar{\partial}_{z} I_{i j}(-\log |z|)^{k\left(v_{i}\right)-k\left(v_{0 j}\right)+1}\right|_{Z, C}<\infty .
$$

Thus we obtain the following convergence:

$$
\begin{aligned}
& \lim _{m \rightarrow \infty}\left\|\bar{z} \cdot \bar{\partial}_{z} I_{i j}^{(m)}(-\log |z|)^{k\left(v_{i}\right)-k\left(v_{0 j}\right)+1}\right\|_{Z, C} \\
& =\lim _{m \rightarrow \infty}\left\|\bar{z} \cdot \bar{\partial}_{z} I_{i j}(-\log |z|)^{k\left(v_{i}\right)-k\left(v_{0 j}\right)+1}\right\|_{Z, C^{m}}=0 .
\end{aligned}
$$

Thus we obtain the following vanishing:

$$
\left\|\bar{z} \cdot \bar{\partial}_{z} I_{i j}^{(\infty)}(-\log |z|)^{k\left(v_{i}\right)-k\left(v_{0 j}\right)+1}\right\|_{Z, C}=0 .
$$

It implies the vanishing $\bar{\partial}_{z} I_{i j}^{(\infty)}=0$. Thus $\mathcal{I}^{(\infty)}$ is holomorphic.

We know that $I_{i j} \cdot(-\log |z|)^{k\left(v_{i}\right)-k\left(v_{0 j}\right)}$ is bounded over $K \times \Delta^{*}$ for any compact subset $K \subset \mathbf{C}_{\lambda}$. Thus $I_{i j}^{(\infty)}$ is dominated by a polynomial of $(-\log |z|)$ on such regions. It implies that $\mathcal{I}^{(\infty)}$ naturally induces the morphism of the prolongments.

When $k\left(v_{i}\right)-k\left(v_{0 j}\right) \neq 0$, we have the finiteness:

$$
\left\|I_{i j}^{(\infty)} \cdot(-\log |z|)^{k\left(v_{i}\right)-k\left(v_{0 j}\right)}\right\|_{W, C}<\infty .
$$

It implies that $I_{i j \mid \mathcal{D}}^{(\infty)}=0$ if $k\left(v_{i}\right)>k\left(v_{0 j}\right)>0$. Namely $\mathcal{I}^{(\infty)}$ preserves the weight filtration on $\mathcal{D}$.

Thus the proof of Proposition 6.1 is completed. q.e.d.

We have the conjugate $\theta^{(\infty) \dagger}$ of $\theta^{(\infty)}$ with respect to the metric $h^{(\infty)}$. We also have the conjugate $\theta_{0}^{(\infty) \dagger}$ of $\theta_{0}^{(\infty)}$ with respect to the metric $h_{0}^{(\infty)}$. 
Corollary 6.3. We have $\theta^{(\infty) \dagger}=\theta_{0}^{(\infty) \dagger}$.

Proof. The holomorphic structures of $\mathcal{F}^{(\infty)}$ and $\mathcal{F}_{0}^{(\infty)}$ are given by the following:

$$
\bar{\partial}_{\lambda}+\bar{\partial}_{F}+\lambda \cdot \theta^{(\infty) \dagger}, \quad \bar{\partial}_{\lambda}+\bar{\partial}_{F}+\lambda \cdot \theta_{0}^{(\infty) \dagger} .
$$

Since the $C^{\infty}$-isomorphism $\mathcal{F}^{(\infty)} \stackrel{=}{\longrightarrow} p_{\lambda}^{-1}(F) \stackrel{=}{\longrightarrow} \mathcal{F}_{0}^{(\infty)}$ is holomorphic, we obtain the equality $\theta^{(\infty) \dagger}=\theta_{0}^{(\infty) \dagger}$.

Corollary 6.4. We have the equality:

$$
\theta^{(\infty)} \cdot \theta^{(\infty) \dagger}+\theta^{(\infty) \dagger} \cdot \theta^{(\infty)}=\theta_{0}^{(\infty)} \cdot \theta_{0}^{(\infty) \dagger}+\theta_{0}^{(\infty) \dagger} \cdot \theta_{0}^{(\infty)} .
$$

We define the section $C \in C^{\infty}\left(\Delta^{*}\right.$, End $\left.(F)\right)$ by $C \cdot d z \cdot d \bar{z}:=\theta^{(\infty)}$. $\theta^{(\infty) \dagger}+\theta^{(\infty) \dagger} \cdot \theta^{(\infty)}$.

By our construction, we have $\mathcal{U}_{0, h}^{0}=\mathcal{U}_{h}^{0}$ on $\mathcal{X}^{0}=\{0\} \times X$.

Lemma 6.17. For any $P \in \Delta^{*}$, the subspace $\mathcal{U}_{0, h \mid(0, P)}^{0}$ is the eigenspace of $C_{\mid(0, P)}$ with the eigenvalue $h \cdot \phi(P)$. Here $\phi(P)$ denotes the function as follows:

$$
-\phi(P)=|z(P)|^{-2} \cdot(-\log |z(P)|)^{-2} \neq 0 .
$$

Proof. Since we have $C \cdot d z \cdot d \bar{z}=\theta_{0}^{(\infty)} \cdot \theta_{0}^{(\infty) \dagger}+\theta_{0}^{(\infty) \dagger} \cdot \theta_{0}^{(\infty)}$, we only have to check the equality in the case of model bundles $\operatorname{Mod}(l+1,1,1)$.

On $\operatorname{Mod}(l+1,1,1)$, we have the canonical frame $\left(e_{1}^{p} \cdot e_{-1}^{q} \mid p+q=l\right)$. Here $\left(e_{1}, e_{-1}\right)$ is the orthogonal frame for $\operatorname{Mod}(2,1,1)$, introduced in Subsubsection 3.2.4. We have the following:

$$
\begin{aligned}
& \theta_{0}\left(e_{1}^{p} \cdot e_{-1}^{q}\right)=p \cdot e_{1}^{p-1} \cdot e_{-1}^{q+1} \cdot \frac{d z}{z} \\
& \theta_{0}^{\dagger}\left(e_{1}^{p} \cdot e_{-1}^{q}\right)=q \cdot e_{1}^{p+1} \cdot e_{-1}^{q-1} \cdot \frac{d \bar{z}}{\bar{z} \cdot(-\log |z|)^{2}} .
\end{aligned}
$$

Thus we obtain the following:

$$
C\left(e_{1}^{p} \cdot e_{-1}^{q}\right)=-(p-q) \cdot e_{1}^{p} \cdot e_{-1}^{q} \cdot \phi(P) .
$$

Hence we are done.

q.e.d.

Lemma 6.18. The endomorphism $C_{\mid P}$ of $E_{\mid P}$ is anti-self adjoint with respect to the metric $h_{\mid P}$. 
Proof. We put $\theta=f \cdot d z$. Let $f^{\dagger}$ denote the adjoint of $f$ with respect to the metric $h$. Then $C_{\mid P}$ is $f \circ f^{\dagger}-f^{\dagger} \circ f$. Thus it is anti-self adjoint.

q.e.d.

In general, the eigenspaces of the anti-self adjoint operator corresponding to the eigenvalues $\alpha_{1}$ and $\alpha_{2}$ are orthogonal, if $\alpha_{1} \neq \alpha_{2}$. Thus we can conclude that $\mathcal{U}_{h \mid P}^{0}$ and $\mathcal{U}_{h^{\prime} \mid P}^{0}$ are orthogonal if $h$ and $h^{\prime}$ are different. Hence the proof of Theorem 6.1 is completed. q.e.d.

\subsubsection{Limiting CVHS in one dimensional case}

We have the following immediate corollary.

Corollary 6.5. Let $\left(E, \bar{\partial}_{E}, \theta, h\right)$ be a tame nilpotent harmonic bundle with trivial parabolic structure over $\Delta^{*}$. Let $\left(F, \theta^{(\infty)}, h^{(\infty)}\right)$ be a limiting harmonic bundle, via the pull backs $\psi_{m, \underline{1}}$. Then $\left(F, \theta^{(\infty)}, h^{(\infty)}\right)$ gives a complex variation of polarized Hodge structure, up to grading.

Proof. We have the decomposition $F=\bigoplus_{h} \mathcal{U}_{h}^{0}$. We have already known that $\mathcal{U}_{h}$ and $\mathcal{U}_{h^{\prime}}$ are orthogonal if $h \neq h^{\prime}$, and $\theta^{(\infty)}\left(\mathcal{U}_{h}\right) \subset \mathcal{U}_{h-2} \otimes$ $\Omega_{\Delta}(\log O)$.

Let $a$ be an element of $S^{1}=\{z \in \mathbf{C}|| z \mid=1\}$. We have the morphism $\rho_{a}: F \longrightarrow F$ given by $\rho_{a}:=\bigoplus_{h} a^{-h} \cdot \operatorname{id}_{\mathcal{U}_{h}^{0}}$. It gives the isomorphism of the Higgs bundle $\left(F, \theta^{(\infty)}\right)$ and $\left(F, a \cdot \theta^{(\infty)}\right)$. It also gives the isomorphism of the hermitian metrics. Hence we obtain the $S^{1}$-action on the harmonic bundle on the harmonic bundle $\left(F, \theta^{(\infty)}, h^{(\infty)}\right)$. Thus it gives a complex variation of the polarized Hodge structures. (See [34] and [35]. See also the Appendix.)

q.e.d.

Let us use the real coordinate $z=r \cdot \exp (\sqrt{-1} \alpha)$.

Proposition 6.2. The $\mathcal{H}(r)$-valued function $H\left(h^{(\infty)}, \boldsymbol{v}^{(\infty)}\right)$ is independent of $\alpha$.

Proof. Consider $H^{(m)}=H\left(\psi_{m}^{*}(h), \boldsymbol{v}^{(m)}\right)$, and the restriction of $H^{(m)}$ to $S_{a}^{1}:=\{z \in \mathbf{C}|| z \mid=a\}$ for $0<a<1$. The components $H_{i j}^{(m)}$ is contained in the image of the linear morphism: $F_{m}: L^{2}\left(S_{a^{n}}^{1}\right) \longrightarrow$ $L^{2}\left(S_{a}^{1}\right)$. The image $J_{m}$ of $F_{m}$ is generated by the following:

$$
\{\exp (\sqrt{-1} h \cdot m \cdot \alpha) \mid h \in \mathbb{Z}\}
$$

Since the intersection $\bigcap_{m_{i}} J_{m_{i}}$ is $\{0\}$, we obtain the result. q.e.d. 


\subsection{The Chern class of the vector bundle $\mathcal{G} r_{h_{1}, h_{2}}^{W\left(N^{\Delta(1)}(\underline{2})\right)}$}

We use the notation in Subsubsection 5.4.3. Consider the case $Q \in$ $D_{\underline{2}}$. We have the vector bundle $\mathcal{G} r_{h_{1}, h_{2}}^{W\left(N^{\triangle(1)}(2)\right)}$ over $\mathbb{P}^{1}$. For later use (Subsection 8.1), we calculate the Chern class of it.

Lemma 6.19. Let $b$ denote the bottom number of $W(\underline{1})$. We have the following:

$$
c_{1}\left(\mathcal{G} r_{b, h_{2}}^{W\left(N^{\triangle(1)}(\underline{2})\right)}\right)=\left(h_{1}+h_{2}\right) \cdot \operatorname{rank} \mathcal{G} r_{b, h_{2}}^{W\left(N^{\triangle(1)}(\underline{2})\right)} .
$$

Proof. We only have to consider the harmonic bundles as in Assumption 6.1 , because the property on $\mathcal{G} r^{\triangle(1)}$ of $S(Q, P)$ is not changed if we take a pull back via $\phi$ given in (55). Thus we assume Assumption 6.1 in the rest of the proof.

Since the first Chern class is topological invariant, it does not depend on a choice of points $P \in X-D$ and $Q \in D_{2}$. We will use it in the following without mention. One immediate consequence is that we only have to consider the case $Q \in D_{\underline{2}}-\bigcup_{i \neq 1,2} D_{\underline{2}} \cap D_{i}$.

Let $(E, \theta, h)$ be a harmonic bundle satisfying Assumption 6.1. We denote the vector bundle $\mathcal{G} r_{h_{1}, h_{2}}^{W\left(N^{\Delta(1)}(\underline{2})\right)}$ for $(E, \theta, h)$ by $\mathcal{G} r_{h_{1}, h_{2}}^{W\left(N^{\Delta(1)}(\underline{2})\right)}(E$, $\theta, h)$ to distinguish the dependence on $(E, \theta, h)$.

Take a limit $\left(F, \theta^{(\infty)}, h^{(\infty)}\right)$ of $(E, \theta, h)$ as in Subsection 6.2. We obtain the vector bundle $\mathcal{G} r_{h_{1}, h_{2}}^{W\left(N^{\Delta(1)}\right)(\underline{2})}\left(F, \theta^{(\infty)}, h^{(\infty)}\right)$.

We need the following lemma.

Lemma 6.20. We have the equality of the Chern classes:

$$
c_{1}\left(\mathcal{G} r_{h_{1}, h_{2}}^{W\left(N^{\triangle(1)}\right)(\underline{2})}\left(F, \theta^{(\infty)}, h^{(\infty)}\right)\right)=c_{1}\left(\mathcal{G} r_{h_{1}, h_{2}}^{W\left(N^{\Delta(1)}\right)(\underline{2})}(E, \theta, h)\right) .
$$

Proof. We denote the vector bundle $S(Q, P)$ for $(E, \theta, h)$ by $S(Q, P$, $(E, \theta, h))$. Let $\boldsymbol{w}$ be a normalizing frame of the deformed holomorphic bundle $(\mathcal{E}, \mathbb{D})$ of $(E, \theta, h)$ over $\mathcal{X}^{\sharp}-\mathcal{D}^{\sharp}$. Assume that $\boldsymbol{w}$ is compatible with the filtration $W(\underline{1})$. We put, as usual, $w_{i}^{(m)}:=\psi_{m}^{*}\left(w_{i}\right) \cdot m^{-k\left(w_{i}\right)}$. Here we put $2 \cdot k\left(w_{i}\right)=\operatorname{deg}^{W(\underline{1})}\left(w_{i}\right)$. Since it gives the normalizing frame of $\left(\psi_{m}^{*} \mathcal{E}, \mathbb{D}^{(m)}\right)$, we obtain the following natural isomorphism:

$$
S\left(Q, P, \psi_{m}^{*}(E, \theta, h)\right) \simeq S\left(Q, \psi_{m}(P),(E, \theta, h)\right) .
$$

The isomorphism preserves the nilpotent maps $N_{1}^{\triangle}$ and $N_{2}^{\triangle}$. Thus we obtain the following:

$$
c_{1}\left(\mathcal{G} r_{h_{1}, h_{2}}^{W\left(N^{\triangle(1)}\right)(\underline{2})}\left(\psi_{m}^{*}(E, \theta, h)\right)\right)=c_{1}\left(\mathcal{G} r_{h_{1}, h_{2}}^{W\left(N^{\triangle(1)}\right)(\underline{2})}(E, \theta, h)\right) .
$$


Pick the subsequence $\left\{m_{i}\right\}$ of $\{m\}$ for the limit $\left(F, \theta^{(\infty)}, h^{(\infty)}\right)$. We can assume that the sequence of the frames $\left\{\Phi_{m_{i}}\left(\boldsymbol{w}^{\left(m_{i}\right)}\right)\right\}$ converges to $\boldsymbol{w}^{(\infty)}$. Then the sequence of the gluings of the bundle $S\left(Q, P, \psi_{m}^{*}(E\right.$, $\theta, h)$ ) converging to the gluings of $S\left(Q, P,\left(F, \theta^{(\infty)}, h^{(\infty)}\right)\right)$. In Lemma 6.9 and Corollary 6.2, we have seen the following:

- The conjugacy class of $N(\underline{1})$ is not changed in the limit.

- The conjugacy class of $N^{(1)}(\underline{2})$ is not changed in the limit.

Then the gluings of the vector bundle $\mathcal{G} r_{h_{1}, h_{2}}^{W\left(N^{\Delta(1)}\right)(2)}\left(\psi_{m_{i}}^{*}(E, \theta, h)\right)$ converges to the gluing of the vector bundle $\mathcal{G} r_{h_{1}, h_{2}}^{W\left(N^{\Delta(1)}\right)(2)}\left(F, \theta^{(\infty)}, h^{(\infty)}\right)$. Since the Chern class is topological invariant, we obtain the following for sufficiently large $m_{i}$ :

$$
c_{1}\left(\mathcal{G} r_{h_{1}, h_{2}}^{W\left(N^{\Delta(1)}\right)(\underline{2})}\left(\psi_{m_{i}}^{*}(E, \theta, h)\right)\right)=c_{1}\left(\mathcal{G} r_{h_{1}, h_{2}}^{W\left(N^{\Delta(1)}\right)(\underline{2})}\left(F, \theta^{(\infty)}, h^{(\infty)}\right)\right) .
$$

Thus the proof of Lemma 6.20 is completed.

q.e.d.

Let us return to the proof of Lemma 6.19. We can assume that the harmonic bundle considered is a limiting harmonic bundle in one direction. Let $Q$ be a point contained in $D_{\underline{2}}$. Consider the tuple of vector space $\mathcal{V}:={ }^{\diamond} \mathcal{E}_{\mid(\lambda, Q)}$ and the nilpotent maps $\mathcal{N}_{1}:=N_{1 \mid(\lambda, Q)}$ and $\mathcal{N}_{2}:=N_{2 \mid(\lambda, Q)}$. The tuple $\left(\mathcal{V}, \mathcal{N}_{1}, \mathcal{N}_{2}\right)$ is decomposed as follows:

Lemma 6.21. There exists the number $M$ and the following data:

- Vector spaces $\mathcal{V}_{i a}$ for $i=1,2$ and for $a=1, \ldots, M$.

- Nilpotent maps $\mathcal{N}_{i a} \in \operatorname{End}\left(\mathcal{V}_{i a}\right)$ for $i=1,2$ and for $a=1, \ldots, M$.

They satisfy the following:

- $\mathcal{V}$ is isomorphic to a direct sum $\bigoplus_{a=1}^{M} \mathcal{V}_{1 a} \otimes \mathcal{V}_{2 a}$.

- Under the isomorphism above, the nilpotent map $\mathcal{N}_{1}$ is same as $\sum_{a=1}^{M} \mathcal{N}_{1 a} \otimes \mathrm{id}_{V_{2 a}}$.

- Under the isomorphism above, the nilpotent map $\mathcal{N}_{2}$ is same as $\sum_{a=1}^{M} \operatorname{id}_{V_{1 a}} \otimes \mathcal{N}_{2 a}$.

Proof. We use the notation in Subsection 6.2.2. We have the decomposition $\diamond \mathcal{E}=\bigoplus_{h} \mathcal{U}_{h}$ satisfying the following:

$$
f_{A_{1}^{(\infty)}}\left(\mathcal{U}_{h}\right) \subset \mathcal{U}_{h-2}, \quad f_{A_{2}^{(\infty)}}\left(\mathcal{U}_{h}\right) \subset \mathcal{U}_{h} .
$$


The space $\bigoplus_{h} \mathcal{U}_{h \mid(\lambda, Q)}$ is naturally isomorphic to the graded associated vector space of the weight filtration of $\mathcal{N}_{1}$. Thus we can decompose $\mathcal{U}_{h \mid(\lambda, Q)}$ into the primitive parts. Since $\mathcal{N}_{1}$ and $\mathcal{N}_{2}$ are commutative, the primitive parts are preserved by $\mathcal{N}_{2}$. Hence we obtain Lemma 6.21.

q.e.d.

Let us compare the filtrations $W(\underline{1})_{\mid(\lambda, Q)}, W(\underline{2})_{\mid(\lambda, Q)}$ of ${ }^{\diamond} \mathcal{E}_{\mid(\lambda, Q)}$, and $W\left(N^{(1)}(\underline{2})\right)_{\mid(\lambda, Q)}$ of $\mathcal{G} r^{(1)}=\mathcal{G} r^{W(\underline{1})}$. For simplicity of the notation, we omit to denote the notation of the restriction ' $\mid(\lambda, Q)^{\prime}$. Let $b$ be the bottom number of the filtration $W(\underline{1})$. Let $v$ be a nonzero element of $W(\underline{1})_{b}$. We have the degree of $v$ with respect to the filtration $W(\underline{2})$, which we denote by $\operatorname{deg}^{W(\underline{2})}(v)$. Since we have the natural isomorphism $W(\underline{1})_{b} \simeq \mathcal{G} r_{b}^{(1)}$, we have the degree of $v \in G r_{b}^{(1)}$ with respect to the filtration $W\left(N^{(1)}(\underline{2})\right)$. The degree is denoted by $\operatorname{deg}^{W\left(N^{(1)}(\underline{2})\right)}(v)$.

Lemma 6.22. We have the following equality:

$$
\operatorname{deg}^{W\left(N^{(1)}(\underline{2})\right)}(v)+b=\operatorname{deg}^{W(\underline{2})}(v) .
$$

Proof. Due to the decomposition as in Lemma 6.21, we only have to consider the case: $\mathcal{V}=\mathcal{V}_{1} \otimes \mathcal{V}_{2}, \mathcal{N}_{1}=\mathcal{N}_{1}^{\prime} \otimes \mathrm{id}_{\mathcal{V}_{2}}$, and $\mathcal{N}_{2}=\mathrm{id}_{\mathcal{V}_{1}} \otimes \mathcal{N}_{2}^{\prime}$. In this case, we can check the equality by using the decomposition as in Subsubsection 2.2.1.

q.e.d.

Thus we obtain the following implication of the vector subbundle over $S(O, P)$ for any point $P$ :

$$
\mathcal{G} r_{b, h_{2}}^{W\left(N^{\triangle(1)}(\underline{2})\right)} \subset \mathcal{G} r_{b+h_{2}}^{W\left(N^{\triangle}(2)\right)} .
$$

Pick an appropriate point $P$ from the curve $\left\{\left(t, t, c_{3}, \ldots, c_{n}\right) \in X-\right.$ $D\}$. Then $S(O, P)$ with the filtration $W^{\triangle}(\underline{2})$ is a mixed twistor. Then $\mathcal{G} r_{b+h_{2}}^{W(\underline{(2}))}$ is isomorphic to a direct sum of $\mathcal{O}_{\mathbb{P}^{1}}\left(b+h_{2}\right)$. Thus we obtain the following inequality:

$$
c_{1}\left(\mathcal{G} r_{b, h_{2}}^{W\left(N^{\triangle(1)}(\underline{2})\right)}\right) \leq\left(b+h_{2}\right) \cdot \operatorname{rank}\left(\mathcal{G} r_{b, h_{2}}^{W\left(N^{\triangle(1)}(\underline{2})\right)}\right) .
$$

On the other hand, we have the following equality:

$$
\sum_{h_{2}} c_{1}\left(\mathcal{G} r_{b, h_{2}}^{W\left(N^{\triangle(1)}(\underline{2})\right)}\right)=c_{1}\left(\mathcal{G} r_{b}^{(1)}\right)=b \cdot \operatorname{rank} \mathcal{G} r_{b}^{(1)}
$$


Note the following equalities:

$$
\begin{aligned}
\sum_{h_{2}} \operatorname{rank}\left(\mathcal{G} r_{b, h_{2}}^{W\left(N^{\Delta(1)}(\underline{2})\right)}\right) & =\operatorname{rank}\left(\mathcal{G} r_{b}^{(1)}\right), \\
\sum_{h_{2}} h_{2} \cdot \operatorname{rank}\left(\mathcal{G} r_{b, h_{2}}^{W\left(N^{\Delta(1)}(\underline{2})\right)}\right) & =0 .
\end{aligned}
$$

Thus we have the following equality:

$$
\sum_{h_{2}} c_{1}\left(\mathcal{G} r_{b, h_{2}}^{W\left(N^{\triangle(1)}(\underline{2})\right)}\right)=\sum_{h_{1}}\left(b+h_{2}\right) \cdot \operatorname{rank}\left(\mathcal{G} r_{b, h_{2}}^{W\left(N^{\triangle(1)}(\underline{2})\right)}\right) .
$$

The inequality (58) and the equality (59) imply the equality (56). Hence the proof of Lemma 6.19 is completed. $\quad$ q.e.d.

\section{The constantness of the filtrations on the positive cones}

\subsection{Preliminary norm estimate}

We put $X=\Delta^{n}=\left\{\left(\zeta_{1}, \ldots, \zeta_{n}\right) \in \Delta^{n}\right\}, D_{i}:=\left\{\zeta_{i}=0\right\}$, and $D=$ $\bigcup_{i=1}^{n} D_{i}$. We also put $D_{\underline{m}}=\bigcap_{j \leq m} D_{j}$. Let $\left(E, \bar{\partial}_{E}, \theta, h\right)$ be a tame nilpotent harmonic bundle with trivial parabolic structure over $X-D$. We have the deformed holomorphic bundle $(\mathcal{E}, \mathbb{D}, h)$ on $\mathcal{X}-\mathcal{D}$, and the prolongment ${ }^{\diamond} \mathcal{E}$. We have the residue $N_{i}:=\operatorname{Res}_{\mathcal{D}_{i}}(\mathbb{D})$. For an element $\boldsymbol{a}=\left(a_{i}\right) \in \mathbf{C}^{n}$, we put $N(\boldsymbol{a})_{\mid(\lambda, O)}:=\sum_{i=1}^{n} a_{i} \cdot N_{i \mid(\lambda, O)}$. We denote the weight filtration of $N(\boldsymbol{a})_{\mid(\lambda, O)}$ by $W(\boldsymbol{a})_{\mid(\lambda, O)}$.

We have already known the following (Proposition 5.5 and Corollary 5.7):

Lemma 7.1. If $N\left(\boldsymbol{a}_{1}\right)_{\mid(\lambda, O)}$ and $N\left(\boldsymbol{a}_{2}\right)_{\mid(\lambda, O)}$ be general in the sense of Definition 2.14 , then $W\left(\boldsymbol{a}_{1}\right)_{\mid(\lambda, O)}=W\left(\boldsymbol{a}_{2}\right)_{\mid(\lambda, O)}$. We also know that if $N\left(\boldsymbol{a}_{1}\right)_{\mid(\lambda, O)}$ is general, then $N\left(\boldsymbol{a}_{1}\right)_{\mid\left(\lambda^{\prime}, O\right)}$ is general for any $\lambda^{\prime}$.

Thus we say $\boldsymbol{a}$ is general if $N(\boldsymbol{a})_{\mid(\lambda, O)}$ is general for all $\lambda$. In the following of this subsection, we fix a general element $\boldsymbol{a}$.

Let $d$ be a positive integer. Let $\boldsymbol{b}_{j}=\left(b_{j 1}, b_{j 2}, \ldots, b_{j n}\right)$ be a general element of $\mathbb{Z}_{>0}^{n}$, for $j=1, \ldots, d$. We put $\tilde{X}=\left\{\left(z_{1}, \ldots, z_{d}\right) \in \Delta^{d}\right\}$, $\widetilde{D}_{i}=\left\{z_{i}=0\right\}$, and $\widetilde{D}=\bigcup_{i=1}^{d} \widetilde{D}_{i}$. We also put $\widetilde{D}_{\underline{m}}=\bigcap_{j \leq m} \widetilde{D}_{j}$. We have the morphism $f: \widetilde{X} \longrightarrow X$ defined as follows:

$$
f^{*}\left(\zeta_{i}\right)=\prod_{j=1}^{d} z_{j}^{b_{j i}}
$$


Note that we have $f\left(\widetilde{D}_{i}\right) \subset D_{\underline{n}}$. We put $(\widetilde{E}, \widetilde{\theta}, \widetilde{h}):=f^{*}(E, \theta, h)$. We also put $(\widetilde{\mathcal{E}}, \widetilde{\mathbb{D}})=f^{*}(\mathcal{E}, \mathbb{D})$.

Let $\boldsymbol{v}$ be a holomorphic frame of ${ }^{\diamond} \mathcal{E}$ over $\mathcal{X}$, which is compatible with the filtration $W(\boldsymbol{a})$ on $\mathcal{D}_{\underline{d}}$. We put $2 \cdot k\left(v_{i}\right)=\operatorname{deg}^{W(\boldsymbol{a})}\left(v_{i}\right)$. We obtain the frame $\widetilde{\boldsymbol{v}}:=f^{*} \boldsymbol{v}$, which gives the frame of ${ }^{\diamond} \widetilde{\mathcal{E}}$. We obtain the $C^{\infty}$-frame $\widetilde{\boldsymbol{v}}^{\prime}$ defined as follows:

$$
\widetilde{v}_{i}^{\prime}:=\left(-\sum_{j=1}^{d} \log \left|z_{j}\right|\right)^{-k\left(v_{i}\right)} \cdot \widetilde{v}_{i} .
$$

Proposition 7.1. The frame $\widetilde{\boldsymbol{v}}^{\prime}$ is adapted over $\Delta_{\lambda}(R) \times(\widetilde{X}-\widetilde{D})$ for any $R>0$.

Proof. We use an induction on $d$. We assume that the claim holds for $d-1$, and we will prove the claim for $d$, in the following. The assumption will be used in Lemma 7.5.

First of all, we note the following: Let $\boldsymbol{v}_{1}$ be another holomorphic frame of $\diamond \mathcal{E}$ over $\mathcal{D}$, which is compatible with the filtration $W(\boldsymbol{a})$. Then we obtain the $C^{\infty}$-frame $\widetilde{\boldsymbol{v}}_{1}^{\prime}$ by the same procedure.

Lemma 7.2. If $\widetilde{\boldsymbol{v}}_{1}^{\prime}$ is adapted over $\Delta_{\lambda}(R) \times(\widetilde{X}-\widetilde{D})$, then $\widetilde{\boldsymbol{v}}^{\prime}$ is adapted over the same region.

Proof. We have the relation $v_{i}=\sum_{j} c_{j i} \cdot v_{1 j}$ over $\mathcal{X}$. Since $\boldsymbol{v}$ and $\boldsymbol{v}_{1}$ are compatible with the filtration $W(\boldsymbol{a})$, we have the following:

$$
\operatorname{deg}^{W(\boldsymbol{a})}\left(v_{i}\right)<\operatorname{deg}^{W(\boldsymbol{a})}\left(v_{1 j}\right) \Longrightarrow c_{j i}(\lambda, O)=0 .
$$

We have the following relation:

$$
\widetilde{v}_{i}^{\prime}=\sum_{j} f^{*}\left(c_{j i}\right) \cdot\left(-\sum_{m=1}^{d} \log \left|z_{m}\right|\right)^{-k\left(v_{i}\right)+k\left(v_{1 j}\right)} \cdot \widetilde{v}_{1 j}^{\prime} .
$$

If $-k\left(v_{i}\right)+k\left(v_{1 j}\right)>0$, then $f^{*}\left(c_{j i}\right)$ is of the form $\left(\prod_{m=1}^{d} z_{m}\right) \cdot g$ for some holomorphic function $g$ over $\widetilde{\mathcal{X}}$. Hence the transformation matrices of $\widetilde{\boldsymbol{v}}^{\prime}$ and $\widetilde{\boldsymbol{v}}_{1}^{\prime}$ are bounded. Thus we obtain Lemma 7.2. q.e.d.

Let us return to the proof of Proposition 7.1. Take a holomorphic frame $\boldsymbol{v}$ of $\diamond \mathcal{E}$ over $\mathcal{X}$, satisfying the following:

- $\boldsymbol{v}_{\mid(\lambda, O)}$ is compatible with the filtration $W(\boldsymbol{a})_{\mid(\lambda, O)}$ for the general $a$. 
- For $\boldsymbol{b}_{1}$, the representing matrix of the endomorphism $\sum_{i} b_{1 i}$. $N_{i \mid(\lambda, O)}$ with respect to the frame $\boldsymbol{v}_{\mid(\lambda, O)}$ is constant, in other words, independent of $\lambda$.

We only have to check the claim of Proposition 7.1 for the frame $\boldsymbol{v}$.

We have the $\lambda$-connection form $\mathcal{A}$ of $\mathbb{D}$ with respect to $\boldsymbol{v}$. We decompose $\mathcal{A}$ as follows:

$$
\mathcal{A}=\sum_{i=1}^{n} A_{i}(\lambda, \zeta) \cdot \frac{d \zeta_{i}}{\zeta_{i}}
$$

We denote the $\lambda$-connection form of $\widetilde{\mathbb{D}}$ with respect to the frame $\widetilde{\boldsymbol{v}}$ by $\widetilde{\mathcal{A}}=\sum \widetilde{\mathcal{A}}_{j}$. We have the following:

$$
\begin{aligned}
\widetilde{\mathcal{A}}=f^{*} \mathcal{A}=\sum_{i=1}^{n} f^{*}\left(A_{i}\right) \cdot f^{*}\left(\frac{d \zeta_{i}}{\zeta_{i}}\right) & =\sum_{i=1}^{n} f^{*}\left(A_{i}\right) \cdot\left(\sum_{j=1}^{d} b_{j i} \cdot \frac{d z_{j}}{z_{j}}\right) \\
& =\sum_{j=1}^{d}\left(\sum_{i=1}^{n} b_{j i} \cdot f^{*} A_{i}\right) \frac{d z_{j}}{z_{j}} .
\end{aligned}
$$

We put $\widetilde{A}_{j}=\sum_{i=1}^{n} b_{j i} \cdot f^{*} A_{i}$. We also have $f\left(\widetilde{D}_{j}\right) \subset\{O\}$. Then we obtain the following relation.

$$
\widetilde{N}_{j}:=\operatorname{Res}_{\widetilde{\mathcal{D}}_{j}}(\widetilde{\mathbb{D}})=\sum_{i=1}^{n} b_{j i} \cdot f^{*}\left(\operatorname{Res}_{\mathcal{D}_{i}}(\mathbb{D})_{\mid(\lambda, O)}\right), \quad(j=1, \ldots, d)
$$

Thus the weight filtration $W\left(\widetilde{N}_{j}\right)$ on $\widetilde{\mathcal{D}}_{j}$ is naturally isomorphic to the pull back of $W\left(\boldsymbol{b}_{j}\right)$ for each $j$.

The residue $\widetilde{N}_{1}$ is represented by the following $M(r)$-valued functions with respect to the frame $\widetilde{\boldsymbol{v}}$ :

$$
\widetilde{A}_{1 \mid \widetilde{\mathcal{D}}_{1}}=\sum_{i=1}^{n} b_{1 i} \cdot A_{i}(\lambda, O) .
$$

Note the following lemma.

Lemma 7.3. $\widetilde{A}_{1 \mid \widetilde{\mathcal{D}}_{j}}$ is constant, say $A$, for any $j=1, \ldots, d$.

Proof. Recall that we have assumed that $\sum_{i=1}^{n} b_{1 i} \cdot A_{i}(\lambda, O)$ is independent of $\lambda$.

q.e.d. 
We put $V={ }^{\diamond} \widetilde{\mathcal{E}}_{\mid(0, \widetilde{O})}$ and $N=\operatorname{Res}_{\widetilde{\mathcal{D}}_{1}}(\widetilde{\mathbb{D}})_{\mid(0, \widetilde{O})}$. We have a model bundle $E(V, N)=\left(E_{0}, h_{0}, \theta_{0}\right)$. We denote the deformed holomorphic bundle by $\left(\mathcal{E}_{0}, \mathbb{D}_{0}, h_{0}\right)$. We have the canonical frame $\boldsymbol{v}_{0}$ such that $\mathbb{D}_{0} \boldsymbol{v}_{0}=\boldsymbol{v}_{0} \cdot A \cdot d z / z$.

Let $q_{1}$ denote the projection $\Delta^{n} \longrightarrow \Delta$ onto the first component. We put $\widetilde{\mathcal{E}}_{0}:=q_{1}^{*} \mathcal{E}_{0}$. We have the $\lambda$-connection $\widetilde{\mathbb{D}}_{0}:=q_{1}^{*} \mathbb{D}_{0}$ along the $z_{1}$-direction. We also put $\widetilde{\boldsymbol{v}}_{0}:=q_{1}^{*} \boldsymbol{v}_{0}$.

Due to the frames $\widetilde{\boldsymbol{v}}$ and $\widetilde{\boldsymbol{v}}_{0}$, we obtain the holomorphic isomorphism $\Phi: \diamond \widetilde{\mathcal{E}}_{0} \longrightarrow{ }^{\diamond} \widetilde{\mathcal{E}}$.

Lemma 7.4. Note the following:

- We have $\Phi \circ \widetilde{\mathbb{D}}_{0}-\mathfrak{q}_{1}(\widetilde{\mathbb{D}}) \circ \Phi=0$ on $\widetilde{\mathcal{D}}_{j}$ for $j=2, \ldots, d$. Similarly we have $\Phi^{-1} \circ \mathfrak{q}_{1}(\widetilde{\mathbb{D}})-\widetilde{\mathbb{D}}_{0} \circ \Phi^{-1}=0$ on $\widetilde{\mathcal{D}}_{j}$ for $j=2, \ldots, d$.

- We have $\operatorname{Res}\left(\Phi \circ \widetilde{\mathbb{D}}_{0}-\mathfrak{q}_{1}(\widetilde{\mathbb{D}}) \circ \Phi\right)=0$ on $\widetilde{\mathcal{D}}_{1}$. Similarly we have $\operatorname{Res}\left(\Phi^{-1} \circ \mathfrak{q}_{1}(\widetilde{\mathbb{D}})-\widetilde{\mathbb{D}}_{0} \circ \Phi^{-1}\right)=0$ on $\widetilde{\mathcal{D}}_{1}$.

Proof. Clear from our construction. (See Subsection 6.1 for $\mathfrak{q}_{1}$.) q.e.d.

We put as follows:

$$
\widetilde{h}_{0}\left(\lambda, z_{1}, \ldots, z_{d}\right):=h_{0}\left(\lambda, \prod_{i=1}^{d} z_{i}\right) .
$$

Proposition 7.1 is a consequence of the following lemma.

Lemma 7.5. The morphisms $\Phi$ and $\Phi^{-1}$ are bounded with respect to the metrics $\widetilde{h}$ and $\widetilde{h}_{0}$, over $\Delta_{\lambda}(R) \times(\widetilde{X}-\widetilde{D})$.

Proof. Let $a$ be an element of $\Delta^{*}$. We put $\widetilde{X}_{a}=\left\{\left(a, z_{2}, \ldots, z_{n}\right) \in\right.$ $\widetilde{X}\}$, and $\widetilde{D}_{a i}:=\widetilde{X}_{a} \cap \widetilde{D}_{i}$. Since $\widetilde{X}_{a}$ is $(d-1)$-dimensional, we can apply the assumption of the induction of the proof of Proposition 7.1 to $\widetilde{X}_{a} \longrightarrow X$. Then we know the following: We have the $C^{\infty}$-frame $\widetilde{\boldsymbol{v}}^{\boldsymbol{\alpha}}$ defined as follows:

$$
\widetilde{v}_{i}^{\boldsymbol{\alpha}}:=\left(-\sum_{j=2}^{d} \log \left|z_{j}\right|\right)^{-k\left(\widetilde{v}_{i}\right)} \cdot \widetilde{v}_{i}
$$

Here we put $2 \cdot k\left(\widetilde{v}_{i}\right)=\operatorname{deg} W(\boldsymbol{a})\left(v_{i}\right)$. Then $\widetilde{\boldsymbol{v}}^{\text {\& }}$ is adapted over $\Delta_{\lambda}(R) \times$ $\left(\widetilde{X}_{a}-\widetilde{D}_{a}\right)$. 
On the other hand, we have the $C^{\infty}$-frame $\widetilde{\boldsymbol{v}}_{0}^{\boldsymbol{\phi}}$ over $\widetilde{X}_{a}$ defined as follows:

$$
\widetilde{v}_{0 i}^{\text {中 }}=\widetilde{v}_{0 i} \cdot\left(-\sum_{j=2}^{d} \log \left|z_{j}\right|\right)^{-k\left(v_{0 i}\right)} \widetilde{v}_{0 i} .
$$

Then it is easy to see that $\widetilde{v}_{0}^{\boldsymbol{h}}$ is adapted over $\Delta_{\lambda}(R) \times\left(\widetilde{X}_{a}-\widetilde{D}_{a}\right)$, due to our construction of $\widetilde{v}_{0}$ and the metric $\widetilde{h}_{0}$.

Thus we obtain the boundedness of the morphisms $\Phi$ and $\Phi^{-1}$ over the boundary $\Delta_{\lambda}(R) \times\left\{\left(z_{1}, \ldots, z_{n}\right) \in \widetilde{X}-\widetilde{D}|| z_{1} \mid=C\right\}$. Then we obtain the boundedness of $\Phi$ and $\Phi^{-1}$ on the region $\Delta_{\lambda}(R) \times\left\{\left(z_{1}, \ldots, z_{n}\right) \in\right.$ $\left.\widetilde{X}-\widetilde{D}|| z_{1} \mid \leq C\right\}$ due to Lemma 6.4. q.e.d.

Thus the induction of the proof of Proposition 7.1 can proceed, namely, the proof of Proposition 7.1 is completed. $\quad$ q.e.d.

\subsection{Taking limit}

\subsubsection{Replacement of Notation}

We essentially use the setting in Subsection 7.1, by putting $d=n$. For simplicity of the notation, we replace $\widetilde{X}$ with $X$, and make similar replacements for others. More precisely, we consider as follows:

We put $X=\Delta^{n}, D_{i}=\left\{z_{i}=0\right\}$ and $D=\bigcup_{i=1}^{n} D_{i}$. Let $\left(E, \bar{\partial}_{E}, \theta, h\right)$ be tame nilpotent harmonic bundle with trivial parabolic structure over $X-D$. As usual, $(\mathcal{E}, \mathbb{D})$ denotes the deformed holomorphic bundle with the $\lambda$-connection. We assume the following:

\section{Condition 7.1.}

- There is a tame nilpotent harmonic bundle $\left(E_{1}, \theta_{1}, h_{1}\right)$ with trivial parabolic structure over $X-D$. We denote the deformed holomorphic bundle by $\left(\mathcal{E}_{1}, \mathbb{D}_{1}\right)$.

- There are general elements $\boldsymbol{b}_{j} \in \mathbb{Z}_{>0}^{n}(j=1, \ldots, n)$, and $f: X \longrightarrow$ $X$ as in Subsection 7.1.

- We have $f^{*}\left(E_{1}, \theta_{1}, h_{1}\right)=(E, \theta, h)$.

- We have a frame $\boldsymbol{v}_{1}$ of $\diamond \mathcal{E}_{1}$ compatible with the filtration $W(\boldsymbol{a})$ on $\mathbf{C}_{\lambda} \times\{O\}$ for general $\boldsymbol{a} \in \mathbf{C}^{n}$. And we have $\boldsymbol{v}=f^{*}\left(\boldsymbol{v}_{1}\right)$. 
By the frame $\boldsymbol{v}$, we decompose ${ }^{\diamond} \mathcal{E}$ as follows:

$$
{ }^{\diamond} \mathcal{E}=\bigoplus_{h} U_{h}, \quad U_{h}:=\left\langle v_{i} \mid \operatorname{deg}^{W\left(N_{1}\right)}\left(v_{i}\right)=h\right\rangle .
$$

We put $2 \cdot k\left(v_{i}\right)=\operatorname{deg}{ }^{W\left(N_{1}\right)}\left(v_{i}\right)$. Recall that the degrees of $v_{i}$ with respect to the weight filtration $W\left(N_{j}\right)$ are independent of $j$, i.e., $\operatorname{deg}^{W\left(N_{j}\right)}\left(v_{i}\right)=\operatorname{deg}^{W\left(N_{1}\right)}\left(v_{i}\right)$.

\subsubsection{Pull backs}

Let $m$ be a nonnegative integers. We have the morphism $\psi_{m, \underline{n}}: X \longrightarrow$ $X$ or $X-D \longrightarrow X-D$, defined as follows:

$$
\psi_{m, \underline{n}}\left(z_{1}, z_{2}, \ldots, z_{n}\right):=\left(z_{1}^{m}, z_{2}^{m}, \ldots, z_{n}^{m}\right) .
$$

Then we obtain the harmonic bundles $\psi_{m, \underline{n}}^{*}\left(E, \bar{\partial}_{E}, \theta, h\right)$ on $X-D$. We also obtain the deformed holomorphic bundles and the $\lambda$-connections $\left(\psi_{m, \underline{n}}^{*} \diamond \mathcal{E}, \psi_{m, \underline{n}}^{*} \mathbb{D}\right)$. We obtain the holomorphic frame $\boldsymbol{v}^{(m)}$ of $\psi_{m, \underline{n}}^{*} \stackrel{\mathcal{E}}{ }$, defined as follows:

$$
v_{i}^{(m)}:=\psi_{m, \underline{n}}^{-1}\left(v_{i}\right) \cdot m^{-k\left(v_{i}\right)} .
$$

We put $H^{(m)}:=H\left(\psi_{m}^{*}(h), \boldsymbol{v}^{(m)}\right)$, which is an $\mathcal{H}(r)$-valued function. The following lemma can be shown by an argument similar to the proof of Lemma 6.7, by using Proposition 7.1.

Lemma 7.6. On any compact subset $K \subset X-D$, the $\mathcal{H}(r)$-valued functions $\left\{H^{(m)}\right\}$ and $\left\{H^{(m)-1}\right\}$ are bounded independently of $m$.

We have the $\lambda$-connection form $\mathcal{A} \in \Gamma\left(\mathcal{X}, M(r) \otimes p_{\lambda}^{*} \Omega_{X}^{1,0}(\log X)\right)$ of $\mathbb{D}$ with respect to the frame $\boldsymbol{v}$. We decompose $\mathcal{A}$ as follows:

$$
\mathcal{A}=\sum_{j} \mathcal{A}_{j}, \quad \mathcal{A}_{j} \in \Gamma\left(\mathcal{X}, M(r) \otimes p_{\lambda}^{*} q_{j}^{*} \Omega_{\Delta}(\log O)\right) .
$$

We obtain $A_{j} \in M(r) \otimes \mathcal{O}_{\mathcal{X}}$ satisfying the following:

$$
\mathcal{A}_{j}=A_{j} \cdot \frac{d z_{j}}{z_{j}}
$$

We decompose $A_{j}$ into $\sum_{h, k} A_{j,(h, k)}$ as in Subsubsection 6.2.2. We have the following:

$$
A_{j,(h, k)}(\lambda, O)=0, \quad \text { if } h>k-2 .
$$


We have the $\lambda$-connection form $\mathcal{A}^{(m)}$ of $\psi_{m, \underline{n}}^{*} \mathbb{D}$ with respect to the frame $\boldsymbol{v}^{(m)}$. We decompose $\mathcal{A}^{(m)}$ into $\sum_{j} \mathcal{A}_{j}^{(m)}$ as in the case of $\mathcal{A}$.

Lemma 7.7. We have the following equalities:

$$
\mathcal{A}_{j}^{(m)}=\sum_{h, k} \psi_{m, \underline{n}}^{*} A_{j(h, k)} \cdot m^{(h-k+2) / 2} \cdot \frac{d z_{j}}{z_{j}} .
$$

In particular, the sequences $\left\{\mathcal{A}^{(m)}\right\}$ converges to $\mathcal{A}^{(\infty)}=\sum_{j} \mathcal{A}_{j}^{(\infty)}$ given as follows:

$$
\mathcal{A}_{j}^{(\infty)}=\sum_{h} A_{j(h, h+2)}(\lambda, O) \cdot \frac{d z_{j}}{z_{j}}
$$

Proof. Similar to Lemma 6.9.

q.e.d.

We decompose $\theta$ into $\sum_{j=1}^{n} f_{j} \cdot d z_{j} / z_{j}$ Then we have $\psi_{m, \underline{n}}^{*} \theta=\sum_{j} m$. $\psi_{m}^{*}\left(f_{j}\right) \cdot d z_{j} / z_{j}$.

Lemma 7.8. We have the following inequality independent of $m$ :

$$
\left|m \cdot \psi_{m}^{*}\left(f_{j}\right)\right|_{\psi_{m}^{*}(h)} \leq C \cdot\left(-\log \left|z_{j}\right|\right)^{-1} .
$$

Proof. Similar to Lemma 6.10.

q.e.d.

In all we obtain the following.

Lemma 7.9. The sequence $\left\{\boldsymbol{v}^{(m)}\right\}$ satisfies Condition 3.2.

\subsubsection{Limit}

We can apply the result in Subsection 3.3 as in Subsubsection 6.2.3. Thus we obtain a limiting harmonic bundle $\left(F, \theta^{(\infty)}, h^{(\infty)}\right)$ and the frame $\boldsymbol{v}^{(\infty)}$ of the prolongment ${ }^{\diamond} \mathcal{F}^{(\infty)}$. We have the following equality:

$$
\mathbb{D}^{(\infty)} \boldsymbol{v}^{(\infty)}=\boldsymbol{v}^{(\infty)} \cdot \mathcal{A}^{(\infty)}, \quad \mathcal{A}^{(\infty)}=\sum_{j} \sum_{h} A_{j(h, h+2)}(\lambda, O) \frac{d z_{j}}{z_{j}}
$$

We have the $C^{\infty}$-frame $\boldsymbol{v}^{\prime(\infty)}$ defined by

$$
v_{i}^{\prime(\infty)}=\left(-\sum_{j=1}^{n} \log \left|z_{j}\right|\right)^{-k\left(v_{i}\right)} \cdot v_{i}^{(\infty)} .
$$

Here $\boldsymbol{v}$ denotes our original frame. 
Lemma 7.10. The frame $\boldsymbol{v}^{\prime(\infty)}$ is adapted.

Proof. Consider the $C^{\infty}$-frame $\boldsymbol{v}^{\prime(m)}$ defined as follows:

$$
v_{i}^{\prime(m)}:=v_{i}^{(m)} \cdot\left(-\sum_{j=1}^{n} \log \left|z_{j}\right|\right)^{-k\left(v_{i}\right)} .
$$

Then $\boldsymbol{v}^{\prime(m)}$ is adapted, independently of $m$. Then the adaptedness of $\boldsymbol{v}^{\prime(\infty)}$ follows immediately.

q.e.d.

We have the real coordinate $z_{i}=r_{i} \cdot \exp \left(2 \pi \sqrt{-1} \alpha_{i}\right)$ for $i=1, \ldots, n$.

Lemma 7.11. The $\mathcal{H}(r)$-valued function $H\left(h^{(\infty)}, \boldsymbol{v}^{(\infty)}\right)$ is independent of $\alpha_{i}$ for any $i$.

Proof. Similar to Proposition 6.2.

q.e.d.

We put $\boldsymbol{e}^{(\infty)}=\boldsymbol{v}_{\mid \mathcal{X}^{0}}^{(\infty)}$. We have the decomposition of $F$ into $\bigoplus_{h} U_{h}$, where $U_{h}$ denote the vector subbundles of $F$ generated by

$$
\left\{e_{i}^{(\infty)} \mid \operatorname{deg} W\left(N_{1}\right)\left(e_{i}\right)=h\right\} .
$$

Note that $A_{j(h, h+2)}$ and $\boldsymbol{e}^{(\infty)}$ determines the morphism $U_{h+2} \longrightarrow U_{h}$.

Pick an element $\boldsymbol{c}=\left(c_{1}, \ldots, c_{n}\right) \in \mathbf{R}_{>0}^{n}$. Then we have the morphism $\Xi_{c}: \mathbb{H} \longrightarrow X-D$ defined as follows:

$$
\Xi_{\boldsymbol{c}}(\zeta)=\left(\exp \left(2 \pi \sqrt{-1} c_{1} \zeta\right), \ldots, \exp \left(2 \pi \sqrt{-1} c_{n} \zeta\right)\right)
$$

We put as follows:

$$
\left(F_{c}, h_{c}, \theta_{c}\right):=\Xi_{c}^{*}\left(F, h^{(\infty)}, \theta^{(\infty)}\right), \quad \boldsymbol{w}_{c}:=\Xi_{c}^{*} e^{(\infty)}, \quad U_{h, c}:=\Xi_{c}^{*} U_{h} .
$$

We use the real coordinate $\zeta=x+\sqrt{-1} y$. We have the $C^{\infty}$-frame $\boldsymbol{w}_{c}^{\prime}$ defined by $w_{\boldsymbol{c} i}^{\prime}:=y^{-k\left(v_{i}\right)} \cdot w_{\boldsymbol{c} i}$. Here $\boldsymbol{v}$ is our original frame.

\section{Lemma 7.12.}

- $H\left(h_{\boldsymbol{c}}, \boldsymbol{w}_{\boldsymbol{c}}\right)$ is independent of $x$.

- We have the following equality:

$$
\theta_{\boldsymbol{c}}\left(\boldsymbol{w}_{\boldsymbol{c}}\right)=\boldsymbol{w}_{\boldsymbol{c}} \cdot\left(\sum_{j} c_{j} \cdot A_{j}^{(\infty)}(0, O)\right) \cdot(2 \pi \sqrt{-1} d \zeta) .
$$


- We have $A_{j}^{(\infty)}(0, O)=\sum_{h} A_{j,(h, h+2)}(0, O)$.

- The frame $\boldsymbol{w}_{c}^{\prime}$ is adapted.

We can descend $\left(F_{\boldsymbol{c}}, h_{\boldsymbol{c}}, \theta_{\boldsymbol{c}}\right)$ to the harmonic bundle over $\Delta^{*}$ by using the framing $\boldsymbol{w}_{\boldsymbol{c}}$. Since $H\left(h_{\boldsymbol{c}}, \boldsymbol{w}_{\boldsymbol{c}}\right)$ does not depend on $x$, it gives the metric $h_{c}$ defined over $\Delta^{*}$. Similarly we obtain the Higgs field $\theta_{c}$ and the vector subbundles $U_{h, \boldsymbol{h}}$ defined over $\Delta^{*}$.

On $\Delta^{*}$, we have the following:

\section{Lemma 7.13.}

- We have the following equality:

$$
\theta_{\boldsymbol{c}}\left(\boldsymbol{w}_{\boldsymbol{c}}\right)=\boldsymbol{w}_{\boldsymbol{c}} \cdot\left(\sum_{j} c_{j} \cdot A_{j}^{(\infty)}(0, O)\right) \cdot \frac{d z}{z} .
$$

In particular, $\theta_{c}$ is tame and nilpotent.

- We have the $C^{\infty}$-frame $\boldsymbol{w}_{c}^{\prime}$ defined by $w_{c i}^{\prime}:=w_{\boldsymbol{c} i} \cdot(-\log |z|)^{-k\left(v_{i}\right)}$, where $\boldsymbol{v}=\left(v_{i}\right)$ is our original frame. Then the frame $\boldsymbol{w}_{\boldsymbol{c}}^{\prime}$ is adapted.

Thus $\boldsymbol{w}_{\boldsymbol{c}}$ naturally gives a frame of ${ }^{\diamond} F_{\boldsymbol{v}}$.

Lemma 7.14. The weight filtration of $\sum_{j} c_{j} \cdot A_{j}^{(\infty)}(0, O)$ is same as the filtration $\left\{W_{l}:=\bigoplus_{h \leq l}{ }^{\diamond} U_{h \mid O} \mid l \in \mathbb{Z}\right\}$.

Proof. We know that $\left|w_{c i}\right|_{h_{c}} \sim(-\log |z|)^{k\left(v_{i}\right)}$, where $\boldsymbol{v}$ is the original frame. Thus the norm estimate in one dimensional case implies our result.

q.e.d.

We put $A_{h, h+2}(\boldsymbol{c}):=\sum_{j} c_{j} \cdot A_{j h h+2}(0, O)$. The endomorphism $N(\boldsymbol{c})_{\mid(0, O)} \in \operatorname{End}\left({ }^{\diamond} \mathcal{E}_{\mid(0, O)}\right)$ induces the morphism $g_{h}(\boldsymbol{c}): G r_{h \mid(0, O)}^{W\left(N_{1}\right)} \longrightarrow$ $G r_{h-2 \mid(0, O)}^{W\left(N_{1}\right)}$. We also have the frame $\boldsymbol{v}^{(1)}$ of $G r_{\mid(0, O)}^{W\left(N_{1}\right)}$ induced by $\boldsymbol{v}$. The matrix $A_{h, h+2}(\boldsymbol{c})$ represents $g_{h+2}(\boldsymbol{c})$ with respect to the frame $\boldsymbol{v}^{(1)}$.

Corollary 7.1. For any $k \geq 0$, the following isomorphism is isomorphic:

$$
g_{-k,-k+2}(\boldsymbol{c}) \circ \cdots \circ g_{k-2, k}(\boldsymbol{c}): G r_{k \mid(0, O)}^{W\left(N_{1}\right)} \longrightarrow G r_{-k \mid(0, O)}^{W\left(N_{1}\right)} .
$$

Proof. We only have to note that ${ }^{\diamond} U_{k \mid O}$ is naturally isomorphic to $G r_{k}^{W\left(N_{1}\right)}$ via the frames $\boldsymbol{v}^{(1)}$ and $\boldsymbol{w}_{\boldsymbol{c}}$.

q.e.d. 
Corollary 7.2. The weight filtration of $N(\boldsymbol{c})_{\mid(\lambda, O)}$ is same as $W\left(N_{1}\right)_{\mid(\lambda, O)}$ for any $\lambda$.

Proof. In the case $\lambda=0$, it is a consequence of Corollary 7.1 and the fact that $N(\boldsymbol{c})_{\mid(0, O)}$ drops the degree with respect to $W\left(N_{1}\right)_{\mid(0, O)}$ by 2. In the other case, we only have to use the fact that the conjugacy classes of $N(\boldsymbol{c})_{\mid(\lambda, O)}$ are independent of $\lambda$.

q.e.d.

\subsection{Constantness of the filtrations on the positive cones}

We put $X=\Delta^{n}, D_{i}=\left\{z_{i}=0\right\}$ and $D=\bigcup_{i=1}^{l} D_{i}$ for $l \leq n$. Let $(E, \theta, h)$ be a tame nilpotent harmonic bundle over $X-D$.

Let $Q$ be a point of $D_{\underline{m}}$. We have the nilpotent maps $N_{j \mid(\lambda, Q)}(j=$ $1, \ldots, m)$. For any element $\boldsymbol{a} \in \mathbf{R}_{\geq 0}^{m}$, we put $N(\boldsymbol{a})_{\mid(\lambda, Q)}:=\sum_{j=1}^{m} a_{j}$. $N_{j \mid(\lambda, Q)}$. Let $W(\boldsymbol{a})_{\mid(\lambda, Q)}$ denote the weight filtration of $N(\boldsymbol{a})_{\mid(\lambda, Q)}$.

Theorem 7.1. The constantness of the filtration on the positive cones holds. Namely we have $W\left(\boldsymbol{a}_{1}\right)_{\mid(\lambda, Q)}=W\left(\boldsymbol{a}_{2}\right)_{\mid(\lambda, Q)}$ for $\boldsymbol{a}_{1}, \boldsymbol{a}_{2} \in$ $\mathbf{R}_{>0}^{I}$, where $I$ denotes any subset of $\underline{m}$.

Proof. We only have to check the following: Assume that $a_{1 j}$ and $a_{2 j}$ are positive for $j=1, \ldots, k$. Assume that $a_{1 j}=a_{2 j}=0$ for $j=k+1, \ldots, l$. Then $W\left(\boldsymbol{a}_{1}\right)_{\mid(\lambda, Q)}=W\left(\boldsymbol{a}_{2}\right)_{\mid(\lambda, Q)}$.

Since such property is not depend on $\lambda$, we only have to check the claim in the case $\lambda=1$. Then, by using the normalizing frame, we know that we have to check such claim when $m=k$, i.e., $Q \in D_{\underline{k}}-\bigcup_{j>k}\left(D_{\underline{k}} \cap\right.$ $\left.D_{j}\right)$.

We only have to check the claim when $m=k=n$. For, we take an $m$-dimensional hyperplane, which intersects with $D_{\underline{m}}$ transversally at $Q$.

Thus we have reduced the theorem to the following claim:

We put $X=\Delta^{n}$ and $D=\bigcup_{i=1}^{n} D_{i}$. Let $\left(E, \bar{\partial}_{E}, \theta, h\right)$ be a tame nilpotent harmonic bundle over $X-D$. Let $O$ be the origin. We have the residues $N_{i \mid(\lambda, O)} i=1, \ldots, n$. We have $W\left(\boldsymbol{a}_{1}\right)_{\mid(\lambda, Q)}=W\left(\boldsymbol{a}_{2}\right)_{\mid(\lambda, Q)}$ if $\boldsymbol{a}_{i} \in \mathbf{R}_{>0}^{n}$.

The following lemma can be shown by an elementary argument.

Lemma 7.15. Let $\boldsymbol{a}$ be an element of $\mathbf{R}_{>0}^{n}$. Then there exists general elements $\boldsymbol{b}_{1}, \ldots, \boldsymbol{b}_{n}$ contained in $\mathbf{Q}_{>0}^{n}$ and positive numbers $\alpha_{1}, \ldots$, 
$\alpha_{n}$ satisfying the following:

$$
\boldsymbol{a}=\sum_{j=1}^{n} \alpha_{j} \cdot \boldsymbol{b}_{j} .
$$

Proof. For example we can argue as follows: Take general elements $\boldsymbol{c}_{j} \in \mathbf{R}_{>0}^{n}$ sufficiently close to $(\overbrace{0, \ldots, 0}^{j-1}, 1, \overbrace{0, \ldots, 0}^{n-j})$. Then $\boldsymbol{a}$ is contained in the positive cone generated by $\boldsymbol{c}_{i}(i=1, \ldots, n)$.

We take $\boldsymbol{d}_{i} \in \mathbf{Q}_{>0}^{n}$ sufficiently close to $\boldsymbol{c}_{i}$. Then $\boldsymbol{d}_{i}$ are general, and $\boldsymbol{a}$ is contained in the positive cone generated by $\boldsymbol{d}_{i}$.

We take $\boldsymbol{b}_{i} \in \mathbb{Z}_{>0}^{n}$ such that $\boldsymbol{b}_{i}=C_{i} \cdot \boldsymbol{d}_{i}$ for some $C_{i} \in \mathbf{R}_{>0}$. Then the tuple $\left(\boldsymbol{b}_{1}, \ldots, \boldsymbol{b}_{n}\right)$ have the desired property. $\quad$ q.e.d.

Then we take the morphism $f: \widetilde{X} \longrightarrow X$ for $\left(\boldsymbol{b}_{1}, \ldots, \boldsymbol{b}_{n}\right)$ as in Subsection 7.1. For the harmonic bundle $f^{*}(E, \theta, h)$, we have already known the constantness of the filtrations (Corollary 7.2). Hence we know that $\boldsymbol{a}$ is general. Thus the proof of Theorem 7.1 is completed.

q.e.d.

We restate a limiting mixed twistor theorem. Let $Q$ be a point of $D=\bigcup_{i=1}^{l} D_{i}$. We put $I=\left\{i \mid Q \in D_{i}\right\}$. Then we put $N^{\triangle}(I):=$ $\sum_{i \in I} N_{i}^{\triangle}$. We denote the weight filtration of $N^{\triangle}(I)$ by $W^{\triangle}(I)$.

\section{Theorem 7.2.}

- Let $U$ be any neighborhood of $Q$. For an appropriate point $P \in$ $U \cap(X-D)$, the filtered vector bundle $\left(S(Q, P), W^{\triangle}(I)\right)$ is a mixed twistor.

- For any $i \in I$, the morphism $N_{i}^{\triangle}: S(Q, P) \longrightarrow S(Q, P) \otimes \mathcal{O}_{\mathbb{P}^{1}}(2)$ is a morphism of mixed twistor.

\section{Strong sequential compatibility}

\subsection{The comparison in the bottom part}

\subsubsection{Two dimensional case}

Let $X$ be $\left\{\left(\zeta_{1}, \zeta_{2}\right) \in \Delta^{2}\right\}, D_{i}=\left\{\zeta_{i}=0\right\}$, and $D=D_{1} \cup D_{2}$. We put $\{O\}=D_{1} \cap D_{2}=D_{\underline{2}}$, and $D_{1}=D_{\underline{1}}$. Let $\left(E, \bar{\partial}_{E}, \theta, h\right)$ be a harmonic 
bundle over $X-D$. We have the deformed holomorphic bundle $(\mathcal{E}, \mathbb{D})$ and the prolongment ${ }^{\diamond} \mathcal{E}$. We put $N_{i}=\operatorname{Res}_{\mathcal{D}_{i}}(\mathbb{D})$. On $\mathcal{D}_{j}$, we put $N(\underline{j})=\sum_{i \leq j} N_{i \mid \mathcal{D}_{\underline{j}}}$ as usual. Let $W(\underline{j})$ denote the weight filtration of $N(\underline{j})$. From the filtration $W(\underline{1})$, we obtain the graded vector bundle $\mathcal{G} r^{(1)}$ over $\mathcal{D}_{\underline{1}}$. On $\mathcal{G} r_{\mid \mathcal{D}_{\underline{\underline{2}}}}^{(1)}$, we have the induced operator $N^{(1)}(\underline{2})$. Thus we obtain the weight filtration $W\left(N^{(1)}(\underline{2})\right)$.

Hence we have the filtrations $W(\underline{1})$ and $W(\underline{2})$ of $\diamond^{\mathcal{E}_{\mid \mathcal{D}_{\underline{2}}}}$. Let $b$ be the bottom number of $W(\underline{1})$. We also have the filtrations $W\left(N^{(1)}(\underline{2})\right)_{h} \cap$ $\mathcal{G} r_{b \mid \mathcal{D}_{\underline{2}}}^{(1)}$ and $W^{(1)}(\underline{2})_{h} \cap \mathcal{G} r_{b \mid \mathcal{D}_{\underline{2}}}^{(1)}$ of $\mathcal{G} r_{b \mid \mathcal{D}_{\underline{2}}}^{(1)}$. We would like to see the relation of them.

Then we have the natural isomorphism $\mathcal{G} r_{b}^{(1)} \simeq W(\underline{1})_{b}$. Thus we obtain the following inclusion:

$$
W\left(N^{(1)}(\underline{2})\right)_{h} \cap \mathcal{G} r_{b \mid \mathcal{D}_{\underline{2}}}^{(1)} \subset W(\underline{1})_{b \mid \mathcal{D}_{\underline{2}}} \subset{ }^{\diamond} \mathcal{E}_{\mid \mathcal{D}_{\underline{2}}} .
$$

We also have the following:

$$
W^{(1)}(\underline{2})_{h} \cap \mathcal{G} r_{b \mid \mathcal{D}_{\underline{2}}}^{(1)}=W(\underline{2})_{h} \cap W(\underline{1})_{b \mid \mathcal{D}_{\underline{2}}} .
$$

Lemma 8.1. We have the following implication:

$$
W\left(N^{(1)}(\underline{2})\right)_{h} \cap \mathcal{G} r_{b \mid \mathcal{D}_{\underline{2}}}^{(1)} \subset W(\underline{2})_{h+b} \cap W(\underline{1})_{b \mid \mathcal{D}_{\underline{2}}} .
$$

Proof. We have already known that both of them are vector bundle over $\mathcal{D}_{2}$ (Subsubsection 5.4.3). Thus we only have to prove the claim on $\mathbf{C}_{\lambda}^{*}$. Pick $\lambda \neq 0$. Take a normalizing frame $\boldsymbol{v}$ of ${ }^{\diamond} \mathcal{E}^{\lambda}$ compatible with the sequence of the filtrations $\left(W(\underline{1}), W\left(N^{(1)}(\underline{2})\right)\right)$. Namely it satisfies the following:

- It is compatible with the filtration $W(\underline{1})$ on $\mathcal{D}_{\underline{1}}$.

- The induced frame $\boldsymbol{v}^{(1)}$ is compatible with $W\left(N^{(1)}(\underline{2})\right)$ on $\mathcal{D}(\underline{2})$.

Let $\mathcal{A}=A_{1} \cdot d \zeta_{1} / \zeta_{1}+A_{2} \cdot d \zeta_{2} / \zeta_{2}$ denote the $\lambda$-connection form of $\mathbb{D}^{\lambda}$ with respect to $\boldsymbol{v}$. Here $A_{i}$ are elements of $M(r)$. We put $U_{b}:=$ $\left\langle v_{i} \mid \operatorname{deg}^{W(\underline{1})}\left(v_{i}\right)=b\right\rangle$, which is a subbundle of ${ }^{\diamond} \mathcal{E}^{\lambda}$.

Lemma 8.2. The vector subbundle $U_{b}$ is stable under the action of $\mathbb{D}^{\lambda}$. Namely $\mathbb{D}^{\lambda}(f)$ is a section of $U_{b}$, if $f$ is a section of $U_{b}$.

Proof. Let $f_{A_{i}}$ be the endomorphism determined by $\boldsymbol{v}$ and $A_{i}$. Then we have $f_{A_{1}}\left(U_{b}\right) \subset U_{b-2}=0$ and $f_{A_{2}}\left(U_{b}\right) \subset U_{b}$. It implies our claim.

q.e.d. 
We put $\widetilde{X}=\Delta^{2}, \widetilde{D}_{i}=\left\{z_{i}=0\right\}$, and $\widetilde{D}=\widetilde{D}_{1} \cup \widetilde{D}_{2}$. Consider the morphism $f: \tilde{X} \longrightarrow X$ defined by $\left(z_{1}, z_{2}\right) \longmapsto\left(z_{1} \cdot z_{2}, z_{2}\right)$. We take the pull back $\left(\widetilde{\mathcal{E}}, \widetilde{\mathbb{D}^{\lambda}}, \widetilde{h}, \widetilde{\boldsymbol{v}}\right):=f^{*}\left(\mathcal{E}^{\lambda}, \mathbb{D}^{\lambda}, h, \boldsymbol{v}\right)$. We also put $\widetilde{U}_{b}:=f^{*} \widetilde{U}_{b}$. Let $\widetilde{\mathcal{A}}$ denote the $\lambda$-connection form of $\widetilde{\mathbb{D}}^{\lambda}$ with respect to $\widetilde{\boldsymbol{v}}$. Then we have the following:

$$
\widetilde{\mathcal{A}}=A_{1} \cdot \frac{d z_{1}}{z_{1}}+\left(A_{1}+A_{2}\right) \cdot \frac{d z_{2}}{z_{2}}
$$

We take the projection $\mathfrak{q}_{2}: \Omega_{X-D}^{1,0} \longrightarrow q_{2}^{*} \Omega_{\Delta^{*}}^{1,0}$. Note that we consider the projection onto the second component, different from the other cases. Then we obtain the $\lambda$-connection along the $z_{2}$-direction: $\mathfrak{q}_{2}\left(\widetilde{\mathbb{D}}^{\lambda}\right)$. By restriction, we have the holomorphic bundle $\widetilde{U}_{b}$ and the $\lambda$-connection $\mathfrak{q}_{2}\left(\widetilde{\mathbb{D}}^{\lambda}\right)$. We put as follows:

$$
\widetilde{\boldsymbol{v}}_{b}:=\left(\widetilde{v}_{i} \mid \operatorname{deg}^{W(\underline{1})}\left(\widetilde{v}_{i}\right)=b\right) .
$$

Then we have $\mathfrak{q}_{2}\left(\widetilde{\mathbb{D}}^{\lambda}\right)\left(\widetilde{\boldsymbol{v}}_{b}\right)=\widetilde{\boldsymbol{v}}_{b} \cdot A_{3} \cdot d z_{2} / z_{2}$ for some constant matrix $A_{3}$.

We put $V=W(\underline{1})_{b \mid(\lambda, O)}$. We have the induced operator $N^{(1)}(\underline{2})$ on $V$. We denote it by $N$. Note that it is represented by the matrix $A_{3}$ for some appropriate base. Then we have a model bundle $E(V, N)=$ $\left(E_{0}, \theta_{0}, h_{0}\right)$. We have the deformed holomorphic bundle $\left(\mathcal{E}_{0}^{\lambda}, \mathbb{D}_{0}^{\lambda}\right)$ on $\Delta^{*}$. We also have the canonical frame $\boldsymbol{v}_{0}$ such that $\mathbb{D}_{0}^{\lambda} \boldsymbol{v}_{0}=\boldsymbol{v}_{0} \cdot A_{3} \cdot d z / z$.

Let $q_{2}$ denote the projection $\Delta_{1}^{*} \times \Delta_{2}^{*} \longrightarrow \Delta_{2}^{*}$ onto the second component. We put $\left(\widetilde{\mathcal{E}}_{0}^{\lambda}, \mathbb{D}_{0}^{\lambda}, \widetilde{\boldsymbol{v}}_{0}\right):=q_{2}^{*}\left(\mathcal{E}_{0}, \mathbb{D}_{0}, \boldsymbol{v}_{0}\right)$. We also put as follows:

$$
\widetilde{h}_{0}\left(\lambda, z_{1}, z_{2}\right):=\left(-\log \left|z_{1}\right|\right)^{b} \cdot h_{0}\left(\lambda, z_{2}\right) \text {. }
$$

Due to the frames $\widetilde{\boldsymbol{v}}_{b}$ and $\widetilde{\boldsymbol{v}}_{0}$, we obtain the holomorphic isomorphism $\Phi: \widetilde{\mathcal{E}}_{0}^{\lambda} \longrightarrow \widetilde{U}_{b}$, satisfying the following:

$$
\Phi \circ \widetilde{\mathbb{D}}_{0}-\mathfrak{q}_{2}(\widetilde{\mathbb{D}}) \circ \Phi=0 .
$$

The morphism $\Phi$ induces the morphism $\widetilde{\mathcal{E}}_{0}^{\lambda} \longrightarrow \widetilde{\mathcal{E}}^{\lambda}$. We denote it by the same notation $\Phi$. It satisfies (61).

Lemma 8.3. The morphism $\Phi$ is bounded with respect to the metrics $\widetilde{h}_{0}$ and $\widetilde{h}$.

Proof. We only have to check the boundedness on the boundary $\left\{\left(z_{1}, z_{2}\right)|| z_{2} \mid=C\right\}$. Let $\widetilde{v}_{i}$ be an element of $\widetilde{\boldsymbol{v}}_{b}$. Then we have 
$\operatorname{deg}^{W(\underline{1})}\left(\widetilde{v}_{i}\right)=b$. Thus we obtain the estimate on the boundary, due to the norm estimate in one dimensional case.

q.e.d.

Let $\widetilde{v}_{i}$ be an element of $\widetilde{\boldsymbol{v}}_{b}$. Due to the lemma, we obtain the following inequality:

$$
\left|\widetilde{v}_{i}\right|_{\widetilde{h}} \leq C \cdot\left(-\log \left|z_{1}\right|\right)^{b / 2} \cdot\left(-\log \left|z_{2}\right|\right)^{k\left(\widetilde{v}_{i}\right)} .
$$

Here we put $2 \cdot k\left(\widetilde{v}_{i}\right):=\operatorname{deg}^{W^{(1)}}(\underline{2})\left(\widetilde{v}_{i}\right)$. Consider the curve $C(1,1)=$ $\{(z, z) \in \widetilde{X}\}$. On the curve $C(1,1)$, we have the following estimate:

$$
\left|\widetilde{v}_{i \mid C(1,1)}\right|_{\tilde{h}} \leq C \cdot(-\log |z|)^{b / 2+k\left(\widetilde{v}_{i}\right)} .
$$

It implies $\widetilde{v}_{i}(O)$ is contained in $W(\underline{2})_{b+2 k\left(\widetilde{v}_{i}\right)}$. Thus we obtain our claim.

q.e.d.

Lemma 8.4. We have $W\left(N^{(1)}(\underline{2})\right)_{h} \cap \mathcal{G} r_{b}^{(1)}=W^{(1)}(\underline{2})_{b+h} \cap \mathcal{G} r_{b}^{(1)}$.

Proof. We will use the mixed twistor structure. Take an appropriate point $P \in \widetilde{X}-\widetilde{D}$ such that the filtration $W(\underline{2})$ gives the mixed twistor structure to the filtered vector bundle $S(O, P)$. Then the filtration $\left\{W^{(1)}(\underline{2})_{h} \cap \mathcal{G} r_{b}^{(1)} \mid h \in \mathbb{Z}\right\}$ gives a mixed twistor structure to $\mathcal{G} r_{b}^{(1)}$. We denote the filtration by $W^{\star}$ for simplicity. The graded vector bundle is denoted by $\mathcal{G} r^{\star}$.

On the other hand, we have the nilpotent map $N^{(1)}(\underline{2}): \mathcal{G} r_{b}^{(1)} \longrightarrow$ $\mathcal{G} r_{b}^{(1)} \otimes \mathcal{O}(2)$. The weight filtration is denoted by $W^{\odot}$, and the associated graded bundle is denoted by $\mathcal{G} r^{\odot}$. Our purpose is to show that $W_{h}^{\odot}=$ $W_{h+b}^{\star}$.

Due to Lemma 8.1, we have already known that $W_{h}^{\odot} \subset W_{h+b}^{\star}$. Due to Lemma 6.19 , we have $c_{1}\left(\mathcal{G} r_{h}^{\mathcal{O}}\right)=(h+b) \cdot \operatorname{rank}\left(\mathcal{G} r_{h}^{\mathcal{P}}\right)$. Then, due to Lemma 2.19 , the vector bundle $\mathcal{G} r_{h}^{\odot}$ naturally gives a vector subbundle of $\mathcal{G} r_{h+b}^{\star}$.

We obtain the inequalities $\operatorname{rank} \mathcal{G} r_{h}^{\odot} \leq \operatorname{rank} \mathcal{G} r_{h+b}^{\star}$. We also have the equalities:

$$
\sum_{h} \operatorname{rank} \mathcal{G} r_{h}^{\bigcirc}=\operatorname{rank} \mathcal{G} r_{b}^{(1)}=\sum_{h} \operatorname{rank} \mathcal{G} r_{h+b}^{\star}
$$

Thus we obtain the equalities $\operatorname{rank} \mathcal{G} r_{h}^{\odot}=\operatorname{rank} \mathcal{G} r_{h+b}^{\star}$. It implies that $W_{h}^{\circlearrowright}=W_{h+b}^{\star}$. 


\subsubsection{Higher dimensional case}

We put $X:=\Delta^{n}, D_{i}=\left\{z_{i}=0\right\}$ and $D=\bigcup_{i=1}^{l} D_{i}$ for some $l \leq n$. Let $\left(E, \bar{\partial}_{E}, \theta, h\right)$ be a tame nilpotent harmonic bundle with trivial parabolic structure over $X-D$. We have the deformed holomorphic bundle with $\lambda$-connection $(\mathcal{E}, \mathbb{D})$ and the prolongment ${ }^{\diamond} \mathcal{E}$. We put $N_{i}=\operatorname{Res}_{\mathcal{D}_{i}}(\mathbb{D})$.

Let $Q$ be a point of $D_{\underline{m}}=\bigcap_{j \leq m} D_{j}$ for $m \leq l$. We put $N(\underline{j})_{\mid(\lambda, Q)}=$ $\sum_{i \leq j} N_{i \mid(\lambda, Q)}$. We denote the weight filtration of $N(\underline{j})_{\mid(\lambda, Q)}$ by $W(\underline{j})_{\mid(\lambda, Q)}$.

Let $b$ be the bottom number of $W(\underline{1})$. On $W(\underline{1})_{b \mid(\lambda, Q)} \simeq \mathcal{G} r_{b \mid(\lambda, Q)}^{(1)}$, we have filtrations $W^{(1)}(\underline{j})_{\mid(\lambda, Q)} \cap \mathcal{G} r_{b \mid(\lambda, Q)}^{(1)}$ and $W\left(N^{(1)}(\underline{j})\right)_{\mid(\lambda, Q)} \cap \mathcal{G} r_{b \mid(\lambda, Q)}^{(1)}$.

Proposition 8.1. We have

$$
W^{(1)}(\underline{j})_{h+b \mid(\lambda, Q)} \cap \mathcal{G} r_{b \mid(\lambda, Q)}^{(1)}=W\left(N^{(1)}(\underline{j})\right)_{h \mid(\lambda, Q)} \cap \mathcal{G} r_{b \mid(\lambda, Q)}^{(1)} .
$$

Proof. We have already known that both of them are vector bundles over $\mathcal{D}_{\underline{m}}$. Thus we only have to see the equality in the case $\lambda \neq 0$.

Take a morphism $\tilde{X} \longrightarrow X$ defined as in Subsection 6.1. Let $\widetilde{Q} \in \widetilde{\mathcal{D}}_{1} \cap \widetilde{\mathcal{D}}_{j}$ be a point such that $\phi(\widetilde{Q})=Q$. Then we have $\widetilde{N}_{1 \mid(\lambda, \widetilde{Q})}$ $:=\operatorname{Res}_{\widetilde{\mathcal{D}}_{1}}(\widetilde{\mathbb{D}})_{\mid(\lambda, \widetilde{Q})}=\phi^{*} N(\underline{1})_{\mid(\lambda, Q)}$, and $\widetilde{N}_{j \mid(\lambda, \widetilde{Q})}:=\operatorname{Res}_{\widetilde{\mathcal{D}}_{j}}(\widetilde{\mathbb{D}})_{\mid(\lambda, \widetilde{Q})}=$ $p h i^{*} N(\underline{j})_{\mid(\lambda, Q)}$. Thus we only have to compare $\widetilde{N}_{1 \mid(\lambda, Q)}$ and $\widetilde{N}_{j \mid(\lambda, Q)}$ at $(\lambda, \widetilde{Q})$.

Since we have assumed $\lambda \neq 0$, we can take a normalizing frame. Then we only have to compare in the case $(\lambda, \widetilde{Q}) \in \widetilde{\mathcal{D}}_{1}^{\lambda} \cap \widetilde{\mathcal{D}}_{j}^{\lambda}-\bigcup_{i \neq j, 1} \widetilde{\mathcal{D}}_{1}^{\lambda} \cap$ $\widetilde{\mathcal{D}}_{j}^{\lambda} \cap \widetilde{\mathcal{D}}_{i}^{\lambda}$.

Take a two dimensional hyperplane of $\widetilde{X}$, which intersects $\widetilde{D}_{1} \cap \widetilde{D}_{j}$ at $\widetilde{Q}$ transversally. Then we can reduce Proposition 8.1 to Lemma 8.4.

q.e.d.

\subsection{Theorem}

We put $X=\Delta^{n}, D_{i}=\left\{z_{i}=0\right\}$, and $D=\bigcup_{i=1}^{l} D$ for $l \leq n$. We put $D_{\underline{m}}=\bigcap_{j \leq m} D_{j}$. Let $\left(E, \bar{\partial}_{E}, \theta, h\right)$ be a tame nilpotent harmonic bundle with trivial parabolic structure, over $X-D$. We have the deformed holomorphic bundle with $\lambda$-connection $(\mathcal{E}, \mathbb{D})$, and the prolongment ${ }^{\diamond} \mathcal{E}$. We put $N_{i}:=\operatorname{Res}_{\mathcal{D}_{i}}(\mathbb{D})$.

Theorem 8.1. The tuple $\left(N_{1}, \ldots, N_{l}\right)$ is sequentially compatible.

Proof. We have already known the following: 
- The conjugacy classes of $N(\underline{j})_{\mid Q}$ is independent of a choice of $Q \in D_{\underline{j}}$ (Corollary 5.7).

- Let $\boldsymbol{h}=\left(h_{1}, \ldots, h_{m}\right)$ be an $m$-tuple of integers. The intersections $\bigcap_{j=1}^{m} W(\underline{j})_{h_{j}}$ form a vector subbundle of ${ }^{\diamond} \mathcal{E}_{\mathcal{D}_{\underline{m}}}$ (Lemma 5.21).

Let $Q$ be a point contained in $D_{\underline{m}}$. We only have to check that $\left(N_{1 \mid(\lambda, Q)}, \ldots, N_{m \mid(\lambda, Q)}\right)$ is sequentially compatible. We have already known that the constantness of the filtration on the positive cone holds (Theorem 7.1).

We use an induction on $l$. Due to our assumption of the induction, we can show that the filtrations $(W(\underline{1}), \ldots, W(\underline{l-1}))$ are compatible by using the normalizing frame. Then we obtain the compatibility of the filtrations $(W(\underline{1}), \ldots, W(\underline{l}))$ by using the mixed twistor structure.

Lemma 8.5. The tuple $\left(N_{2}^{(1)}, \ldots, N_{l}^{(1)}\right)$ is sequentially compatible.

Proof. Take a limiting harmonic bundle in one dimensional direction $\left(F, \theta^{(\infty)}, h^{(\infty)}\right)$ of $\left(E, \bar{\partial}_{E}, \theta, h\right)$. We have the deformed holomorphic bundle $\left(\mathcal{F}^{(\infty)}, \mathbb{D}^{(\infty)}\right)$. Pick $a \in \Delta^{*}$. We put $X_{a}:=\{a\} \times \Delta^{n-1} \subset \Delta^{n}=X$, and $D_{a i}:=D_{i} \cap X_{a}$ for $i=2, \ldots, l$. We have $\mathcal{X}_{a} \subset \mathcal{X}$ and $\mathcal{D}_{a i}=\mathcal{D}_{i} \cap \mathcal{X}_{a}$. We have the residues $N_{a i}^{(\infty)}:=\operatorname{Res}_{\mathcal{D}_{a i}}\left(\mathbb{D}_{\mid \mathcal{X}_{a}}^{(\infty)}\right)$ for $i=2, \ldots, l$. By our assumption of the induction on $l$, we have already known that $\left(N_{a 2}^{(\infty)}, \ldots, N_{a l}^{(\infty)}\right)$ is sequentially compatible.

On the other hand, we have already seen that the tuple of the vector space ${ }^{\diamond} \mathcal{F}^{(\infty) \lambda}{ }_{\mid(\lambda, Q)}$ the residues $\operatorname{Res}_{\mathcal{D}_{i}}\left(\mathbb{D}_{\mid \mathcal{X}_{a}^{\lambda}}^{(\infty) \lambda}\right)$ are isomorphic to the tuple of the graded vector space $\mathcal{G} r^{(1)}$ and the residues $N_{i}^{(1)}$, due to Lemma 6.9 and Corollary 6.2. Thus we obtain our claim. q.e.d.

We know that $\left(N_{1}, \ldots, N_{l}\right)$ is sequentially compatible in the bottom part, due to Proposition 8.1. Proposition 8.1 holds for any tame nilpotent harmonic bundle with trivial parabolic structure. Thus we obtain the universal sequentially compatibility in the bottom part. Then we obtain the sequentially compatibility due to Proposition 2.1. q.e.d.

Theorem 8.2. The tuple $\left(N_{1}, \ldots, N_{l}\right)$ is strongly sequentially compatible.

Proof. Let $Q$ be a point contained in $D_{\underline{m}}$. We only have to check that $\left(N_{1 \mid(\lambda, Q)}, \ldots, N_{m \mid(\lambda, Q)}\right)$ is strongly sequentially compatible. We use an induction on $m$. We assume that the claim for $m-1$ holds, and then we will prove that the claim for $m$ holds. 
Assume that $Q$ is contained in $D_{m}-\bigcup_{h>m}\left(D_{m} \cap D_{h}\right)$. Then we can pick a point $P \in X-D$ such that the filtration $W^{\triangle}(\underline{m})$ of $S(Q, P)$ is a mixed twistor. By our assumption of induction, the sequence $\left(N_{1 \mid(\lambda, Q)}, \ldots, N_{m-1 \mid(\lambda, Q)}\right)$ is strongly sequentially compatible. We have already proved that the sequence $\left(N_{1 \mid(\lambda, Q)}, \ldots, N_{m-1 \mid(\lambda, Q)}, N_{m \mid(\lambda, Q)}\right)$ is sequentially compatible (Theorem 8.1). Thus we obtain that $\left(N_{1 \mid(\lambda, Q)}\right.$, $\left.\ldots, N_{m \mid(\lambda, Q)}\right)$ is strongly sequentially compatible in this case, due to Proposition 2.3.

Consider the case that $Q$ is contained in $\bigcup_{h>m}\left(D_{\underline{m}} \cap D_{h}\right)$. Pick $\lambda \neq$ 0 . Using a normalizing frame, we obtain that $\left(N_{1 \mid(\lambda, Q)}, \ldots, N_{m-1 \mid(\lambda, Q)}\right.$, $\left.N_{m \mid(\lambda, Q)}\right)$ is strongly sequentially compatible, if $\lambda \neq 0$. We put $I=$ $\left\{i \mid D_{i} \ni Q\right\}$. Then we can pick an appropriate $P$ such that $(S(Q, P)$, $\left.W^{\triangle}(I)\right)$ is mixed twistor. By using the mixed twistor structure, we can conclude that $\left(N_{1 \mid(0, Q)}, \ldots, N_{m \mid(0, Q)}\right)$ is also strongly sequentially compatible.

Thus the induction on $m$ can proceed, namely the proof of Theorem 8.2 is completed.

q.e.d.

Theorem 8.3. The tuple $\left(N_{1}, \ldots, N_{l}\right)$ is of Hodge.

Proof. For any $\sigma \in \mathfrak{S}_{l}$, the tuple $\left(N_{\sigma(1)}, \ldots, N_{\sigma(l)}\right)$ is strongly sequentially compatible. Thus we are done.

q.e.d.

\section{Norm estimate and limiting CVHS in higher dimensional case}

\subsection{Norm estimate}

\subsubsection{Preliminary norm estimate}

We put $X=\Delta^{n}=\left\{\left(\zeta_{1}, \ldots, \zeta_{n}\right)\right\}, D_{i}=\left\{\zeta_{i}=0\right\}$ and $D=\bigcup_{i=1}^{l} D_{i}$ for any $l \leq n$. Let $\left(E, \bar{\partial}_{E}, \theta, h\right)$ be a tame nilpotent harmonic bundle with trivial parabolic structure over $X-D$. We have the deformed holomorphic bundle with $\lambda$-connection $(\mathcal{E}, \mathbb{D})$ and the prolongment ${ }^{\diamond} \mathcal{E}$. We put $N_{i}=\operatorname{Res}_{\mathcal{D}_{i}}(\mathbb{D})$. Then $\left(N_{1}, \ldots, N_{n}\right)$ gives a strongly sequentially compatible tuple.

Let $\boldsymbol{v}$ be a frame of ${ }^{\diamond} \mathcal{E}$ over $\mathcal{X}$ which is compatible with $\left(N_{1}, \ldots, N_{n}\right)$. We put as follows:

$$
2 \cdot k_{j}\left(v_{i}\right)=\operatorname{deg}^{W(\underline{j})}\left(v_{i}\right)-\operatorname{deg} W(\underline{j-1})\left(v_{i}\right)
$$


We put $\widetilde{X}:=\left\{\left(z_{1}, \ldots, z_{n}\right) \in \Delta^{n}\right\}, \widetilde{D}_{i}:=\left\{z_{i}=0\right\}$, and $\widetilde{D}=\bigcup_{i=1}^{l} \widetilde{D}_{i}$. Let $(c(1), \ldots, c(n))$ be a sequence of integers such that $0 \leq c(1)<$ $c(2)<\cdots<c(n)$. Let $N$ be any positive integer. Consider the morphism $\phi_{N}: \widetilde{X} \longrightarrow X$ defined as follows:

$$
\phi_{N}^{*}\left(\zeta_{i}\right)= \begin{cases}\left(\prod_{j=i}^{l} z_{j}\right)^{N^{c(i)}} & (i \leq l) \\ z_{i} & (i \geq l+1) .\end{cases}
$$

We put $(\widetilde{\mathcal{E}}, \widetilde{\mathbb{D}}, \widetilde{h})=\phi_{N}^{*}(\mathcal{E}, \mathbb{D}, h)$. We also put $\widetilde{\boldsymbol{v}}=\phi_{N}^{*} \boldsymbol{v}$. We have the $C^{\infty}$-frame $\widetilde{\boldsymbol{v}}^{\prime}$ defined as follows:

$$
\widetilde{v}_{i}^{\prime}:=\widetilde{v}_{i} \cdot \prod_{m=1}^{l}\left(-\sum_{t=m}^{l} \log \left|z_{t}\right|\right)^{-k_{m}\left(v_{i}\right)} .
$$

Proposition 9.1. The frame $\widetilde{\boldsymbol{v}}^{\prime}$ is adapted over $\Delta_{\lambda}(R) \times(\widetilde{X}-\widetilde{D})$ for any $R>0$.

Proof. We use an induction on $l$. We assume that the claim for $l-1$ holds and we will prove the claim for $l$. The assumption of the induction will be used in Lemma 9.2.

First we note the following: Let $\boldsymbol{v}_{1}$ be another frame compatible with the sequence $\left(N_{1}, \ldots, N_{l}\right)$. By the same procedure, we obtain the $C^{\infty}$-frame $\widetilde{\boldsymbol{v}}_{1}^{\prime}$.

Lemma 9.1. If $\widetilde{\boldsymbol{v}}_{1}^{\prime}$ is adapted over $\Delta_{\lambda}(R) \times(\widetilde{X}-\widetilde{D})$, then $\widetilde{\boldsymbol{v}}^{\prime}$ is also adapted over the same region.

Proof. We have the relation $v_{i}=\sum_{j} b_{j i} \cdot v_{1 j}$. Since $\boldsymbol{v}$ and $\boldsymbol{v}_{1}$ are compatible with the sequence $\left(N_{1}, \ldots, N_{l}\right)$, we have the following:

$$
\operatorname{deg}^{W}(\underline{m})\left(v_{i}\right)<\operatorname{deg}^{W(\underline{m})}\left(v_{1 j}\right) \Longrightarrow b_{j i \mid \mathcal{D}_{\underline{m}}}=0 .
$$

If we put $m_{0}(i, j)=\min \left\{m \mid k_{m}\left(v_{i}\right)<k_{m}\left(v_{1 j}\right)\right\}$, then we obtain that $b_{j i \mid D_{m_{0}(i, j)}}=0$. Since we have $\phi_{N}\left(\widetilde{\mathcal{D}}_{t}\right) \subset \mathcal{D}_{\underline{m_{0}(i, j)}}$ for $t \geq m_{0}(i, j)$, the holomorphic function $\phi_{N}^{*} b_{j i}$ is of the form $g \cdot \prod_{t=m_{0}(i, j)}^{l} z_{t}$ for some holomorphic function $g$.

We have the following relation:

$$
\widetilde{v}_{i}^{\prime}=\sum_{j} \widetilde{B}_{j i}^{\prime} \cdot \widetilde{v}_{1 j}^{\prime}, \quad \widetilde{B}_{j i}^{\prime}=\phi_{N}^{*}\left(b_{j i}\right) \cdot \prod_{m=1}^{l}\left(-\sum_{t=m}^{l} \log \left|z_{t}\right|\right)^{-k_{m}\left(v_{i}\right)+k_{m}\left(v_{1 j}\right)} .
$$


Thus $\widetilde{B}_{j i}^{\prime}$ is bounded over $\Delta_{\lambda}(R) \times(\widetilde{X}-\widetilde{D})$ for any $R>0$. Thus $\widetilde{B}^{\prime}$ is bounded over the same region. Similarly, the boundedness of $\widetilde{B}^{\prime-1}$ over the same region can be shown. Thus the proof of Lemma 9.1 is completed.

q.e.d.

Let $\boldsymbol{v}$ be a frame of $\diamond \mathcal{E}$ over $\mathcal{X}$, which is strongly compatible with the tuple $\left(N_{1}, \ldots, N_{l}\right)$ (see Subsubsection 2.4.3 and Definition 2.29). Then $\boldsymbol{v}$ satisfies the following:

- $\boldsymbol{v}=\left(v_{k, \boldsymbol{h}, \eta} \mid k \geq 0, \boldsymbol{h} \in \mathbb{Z}^{l}, \eta=1, \ldots, d(k, \boldsymbol{h})\right)$.

- $2 \cdot k_{j}\left(v_{k, \boldsymbol{h}, \eta}\right)=h_{j}$.

- We put $\boldsymbol{\delta}=(1,0, \ldots, 0)$. For an element $\boldsymbol{h}$, the first component is denoted by $h_{1}$. Then we have the following:

$$
N_{1}\left(v_{k, \boldsymbol{h}, \eta}\right)= \begin{cases}v_{k, \boldsymbol{h}-2 \cdot \boldsymbol{\delta}_{1}, \eta} & \left(h_{1}>-k\right) \\ 0 & \left(h_{1}=-k\right) .\end{cases}
$$

We only have to prove the claim of Proposition 9.1 for such a frame $\boldsymbol{v}$.

As a preliminary consideration, we see the frame $\widetilde{\boldsymbol{v}}^{\circ}$ defined as follows:

$$
\widetilde{v}_{k, \boldsymbol{h}, \eta}^{\circ}:=\widetilde{v}_{k, \boldsymbol{h}, \eta} \cdot\left(-\sum_{j=2}^{l} \log \left|z_{j}\right|\right)^{-\left(h_{1}+h_{2}\right) / 2} \cdot \prod_{i=3}^{l}\left(-\sum_{j=i}^{l} \log \left|z_{j}\right|\right)^{-h_{i} / 2} .
$$

Lemma 9.2. Let $C$ be a real number such that $0<C<1$. On $\Delta_{\lambda}(R) \times\left\{\left(z_{1}, \ldots, z_{n}\right) \in \widetilde{X}-\widetilde{D}|| z_{1} \mid=C\right\}$, the frame $\widetilde{\boldsymbol{v}}^{\circ}$ is adapted for any $R>0$.

Proof. Take $a \in \mathbf{C}$ such that $|a|=C$. We put $\widetilde{X}_{a}:=\left\{\left(a, z_{2}, \ldots\right.\right.$, $\left.\left.z_{n}\right) \in \widetilde{X}\right\}$ and $\widetilde{D}_{a i}:=\widetilde{D}_{i} \cap \widetilde{X}_{a}$. Then $\widetilde{D}_{a}=\widetilde{D} \cap X=\bigcup_{i=2}^{l} \widetilde{D}_{a, i}$. We also put $X_{a}:=\left\{\left(\zeta_{1}, \ldots, \zeta_{n}\right) \in X \mid \zeta_{1}^{N^{c(2)-c(1)}}=a^{N^{c(2)-c(1)}} \zeta_{2}\right\}$ and $D_{a i}=$ $D_{i} \cap X_{a}$. Then $D_{a}=D \cap X=\bigcup_{i=2}^{l} D_{a, i}$. We have the isomorphisms:

$$
\widetilde{X}_{a} \simeq\left\{\left(z_{2}, \ldots, z_{n}\right) \in \Delta^{n-1}\right\}, \quad X_{a} \simeq\left\{\left(\zeta_{1}, \zeta_{3}, \ldots, \zeta_{n}\right) \in \Delta^{n-1}\right\} .
$$

The restriction of $\phi_{N}$ gives the morphism $\tilde{X}_{a} \longrightarrow X_{a}$. Applying the assumption of the induction, we obtain the result.

We have the $\lambda$-connection along the $z_{1}$-direction $\mathfrak{q}_{1}(\widetilde{\mathbb{D}})$ (Subsection 6.1 ). We have the $\lambda$-connection form $A \cdot d z_{1} / z_{1}$ of $\mathfrak{q}_{1}(\widetilde{\mathbb{D}})$ with 
respect to the frame $\widetilde{\boldsymbol{v}}$. We have the decomposition of the frame as follows:

$$
\widetilde{\boldsymbol{v}}=\bigcup_{k \geq 0, \boldsymbol{l} \in \mathbb{Z}^{l-1}}\left(v_{k, \boldsymbol{h}, \eta} \mid \pi_{1}(\boldsymbol{h})=\boldsymbol{l}, \eta=1, \ldots, d(k, \boldsymbol{h})\right) .
$$

Here we put $\pi(\boldsymbol{h})=\left(h_{2}, \ldots, h_{n}\right)$ for $\boldsymbol{h}=\left(h_{1}, h_{2}, \ldots, h_{n}\right)$. Corresponding to the decomposition of the frame, we obtain the decomposition of $\diamond \mathcal{E}$ :

$$
\diamond \mathcal{E}=\bigoplus_{k \geq 0} \bigoplus_{l \in \mathbb{Z}^{l-1}} \mathcal{L}_{k, l}
$$

\section{Lemma 9.3.}

- The restrictions $A_{\mid \widetilde{\mathcal{D}}_{i}}(i=1, \ldots, l)$ are constant.

- Corresponding to the decomposition of $\diamond \mathcal{E}$, it is decomposed into $A_{k, \boldsymbol{l}}$ for $k \geq 0$, and $\boldsymbol{l} \in \mathbb{Z}^{l-1}$.

Proof. The first claim is clear from our construction. The second claim corresponds to the fact that $N_{1}$ preserves the bundles $\mathcal{L}_{k, l}$ for $k \geq 0$ and $\boldsymbol{l} \in \mathbb{Z}^{l-1}$.

For $k \geq 0$ and $\boldsymbol{l} \in \mathbb{Z}^{l-1}$, we put $V_{k, l}:=\mathcal{L}_{k, l \mid(0, O)}$. The morphism $N_{1}$ induces $N_{k, l}$ on $\mathcal{V}_{k, l}$. We have a harmonic bundle $E\left(V_{k, l}, N_{k, l}\right)=$ $\left(E_{0, k, l}, \theta_{0, k, l}, h_{0, k, l}\right)$. We have the deformed holomorphic bundle $\left(\mathcal{E}_{0, k, l}\right.$, $\left.\mathbb{D}_{0, k \boldsymbol{l}}\right)$. We have the canonical frame $\boldsymbol{v}_{0, k, \boldsymbol{l}}=\left(v_{0, k, \boldsymbol{h}, \eta} \mid \pi_{1}(\boldsymbol{h})=\boldsymbol{l}, \eta=\right.$ $1, \ldots, d(k, \boldsymbol{h}))$ satisfying $\mathbb{D}_{0, k, \boldsymbol{l}} \boldsymbol{v}_{0, k, \boldsymbol{l}}=\boldsymbol{v}_{0, k, \boldsymbol{l}} \cdot A_{k, \boldsymbol{l}} d z / z$.

Let $q_{1}$ denote the projection $\Delta^{n} \longrightarrow \Delta$ onto the first component. We put $\left(\widetilde{\mathcal{E}}_{0, k, \boldsymbol{l}}, \widetilde{\mathbb{D}}_{0, k, \boldsymbol{l}}, \widetilde{\boldsymbol{v}}_{0, k, \boldsymbol{l}}\right)=q_{1}^{*}\left(\mathcal{E}_{0, k \boldsymbol{l}}, \mathbb{D}_{0, k, \boldsymbol{l}}, \boldsymbol{v}_{0, k, \boldsymbol{l}}\right)$. We also put as follows:

$$
\widetilde{h}_{0, k, l}\left(z_{1}, \ldots, z_{n}\right)=\left[\prod_{i=2}^{l}\left(-\sum_{j=i}^{l} \log \left|z_{j}\right|\right)^{l_{i-1} / 2}\right] \cdot h_{0, k, l}\left(\prod_{j=1}^{l} z_{j}\right) .
$$

Consider the frame $\widetilde{\boldsymbol{v}}_{0, k, l}^{\star}$ defined as follows:

$$
\widetilde{v}_{0, k, \boldsymbol{h}, \eta}^{\star}:=\widetilde{v}_{0, k, \boldsymbol{h}, \eta} \cdot\left(-\sum_{j=2}^{l} \log \left|z_{j}\right|\right)^{-\left(h_{1}+h_{2}\right) / 2} \cdot \prod_{i=3}^{l}\left(-\sum_{j=i}^{l} \log \left|z_{j}\right|\right)^{-h_{i} / 2} .
$$

Note that we have $h_{i}=l_{i-1}$ by definition. 
Lemma 9.4. Let $C$ be a real number such that $0<C<1$. The $C^{\infty}$-frame $\boldsymbol{v}_{0, k, l}^{\star}$ is adapted over $\Delta_{\lambda}(R) \times\left\{\left(z_{1}, \ldots, z_{n}\right) \in \widetilde{X}-\widetilde{D}|| z_{1} \mid=\right.$ $C\}$ for any $R>0$.

Proof. Clear from our construction.

q.e.d.

We put as follows:

$$
\left(\widetilde{\mathcal{E}}_{0}, \widetilde{\mathbb{D}}_{0}, \widetilde{h}_{0}, \widetilde{\boldsymbol{v}}_{0}\right):=\bigoplus_{k \geq 0} \bigoplus_{\boldsymbol{l} \in \mathbb{Z}^{n-1}}\left(\widetilde{\mathcal{E}}_{0, k, l}, \widetilde{\mathbb{D}}_{0, k, l}, \widetilde{h}_{0, k, \boldsymbol{l}}, \widetilde{\boldsymbol{v}}_{0, k, l}\right) .
$$

By using the frames $\widetilde{\boldsymbol{v}}_{0}$ and $\widetilde{\boldsymbol{v}}$, we obtain the holomorphic isomorphism $\Phi: \widetilde{\mathcal{E}}_{0} \longrightarrow \widetilde{\mathcal{E}}$. The proof of Proposition 9.1 is reduced to the following lemma:

Lemma 9.5. The morphisms $\Phi$ and $\Phi^{-1}$ are bounded with respect to the metrics $\widetilde{h}$ and $\widetilde{h}_{0}$, over $\Delta_{\lambda}(R) \times(\widetilde{X}-\widetilde{D})$ for any $R>0$.

Proof. We only have to check the boundedness on the boundary $\Delta_{\lambda}(R) \times\left\{\left(z_{1}, \ldots, z_{n}\right) \in \widetilde{X}-\widetilde{D}|| z_{1} \mid=C\right\}$, which we have already seen (Lemma 9.2 and Lemma 9.4).

q.e.d.

Thus we obtain the adaptedness of the frame $\widetilde{\boldsymbol{v}}$. The induction on $l$ can proceed, namely the proof of Proposition 9.1 is completed. q.e.d.

\subsubsection{Replacement of Notation}

We put $X=\left\{\left(z_{1}, \ldots, z_{n}\right) \in \Delta^{n}\right\}, D_{i}=\left\{z_{i}=0\right\}$, and $D=\bigcup_{i=1}^{l} D_{i}$ for $l \leq n$. Let $\left(E, \bar{\partial}_{E}, \theta, h\right)$ be a tame nilpotent harmonic bundle with trivial parabolic structure over $X-D$. We have the deformed holomorphic bundle with $\lambda$-connection $(\mathcal{E}, \mathbb{D})$ and the prolongment ${ }^{\diamond} \mathcal{E}$. We put $N_{i}=$ $\operatorname{Res}_{\mathcal{D}_{i}}(\mathbb{D})$. We take a holomorphic frame $\boldsymbol{v}$ of ${ }^{\diamond} \mathcal{E}$, which is compatible with $\left(N_{1}, \ldots, N_{l}\right)$. We take the $C^{\infty}$-frame $\boldsymbol{v}^{\prime}$ defined as follows:

$$
v_{k, \boldsymbol{h}, \eta}^{\prime}=v_{k, \boldsymbol{h}, \eta} \cdot \prod_{i=1}^{l}\left(-\log \left|z_{i}\right|\right)^{-h_{i} / 2} .
$$

Then the following theorem is the reformulation of Proposition 9.1 when we put $c(i)=i-1$.

Theorem 9.1. Let $N$ be any positive integer. Consider the following region:

$$
Z(\mathrm{id}, l, N):=\left\{\left.\left(z_{1}, \ldots, z_{n}\right)|| z_{i-1}\right|^{N}<\left|z_{i}\right|, i=2, \ldots, l\right\} .
$$

On $Z(\mathrm{id}, l, N)$, the frame $\boldsymbol{v}^{\prime}$ is adapted. 
Proof. By the morphism $\phi_{N}$, we have the isomorphism of the following regions:

$$
\Delta^{* l} \times \Delta^{n-l} \stackrel{\phi_{N} \simeq}{\longrightarrow} Z(\mathrm{id}, l, N) .
$$

Moreover, we have the following replacement:

$$
-N^{i-1} \sum_{j=i}^{l} \log \left|z_{j}\right| \stackrel{\phi_{N}}{\longleftrightarrow}-\log \left|z_{i}\right|, \quad(i \leq l) .
$$

Here we put $c(i)=i-1$. Thus we are done.

q.e.d.

\subsubsection{Theorem}

Let $\sigma$ be an element of $\mathfrak{S}_{l}$, and we put $I_{j}=\{\sigma(i) \mid i \leq j\}$ for $j=1, \ldots, l$. We put $\mathcal{D}_{I_{j}}=\bigcap_{i \in I} \mathcal{D}_{i}$. On $\mathcal{D}_{I_{j}}$, we put $N\left(I_{j}\right):=\sum_{i \in I_{j}} N_{i \mid \mathcal{D}_{I_{j}}}$. We denote the weight filtration of $N\left(I_{j}\right)$ by $W\left(I_{j}\right)$. Then we obtain the strongly sequentially compatible commuting tuple $\left(N_{\sigma(1)}, \ldots, N_{\sigma(l)}\right)$ and the compatible sequence of the filtrations $\left(W\left(I_{1}\right), W\left(I_{2}\right), \ldots, W\left(I_{l}\right)\right)$.

Let $\boldsymbol{v}$ be a holomorphic frame of ${ }^{\diamond} \mathcal{E}$ which is compatible with the sequentially compatible tuple $\left(N_{\sigma(1)}, \ldots, N_{\sigma(l)}\right)$. Recall that we have the number $\operatorname{deg}^{W(I(j))}\left(v_{i}\right)$ for any $v_{i}$ and any $j=1, \ldots, l$. We put as follows:

$$
2 \cdot k_{j}\left(v_{i}\right):=\operatorname{deg}^{W(I(j))}\left(v_{i}\right)-\operatorname{deg} W(I(j-1))\left(v_{i}\right) .
$$

We obtain the $C^{\infty}$-frame $\boldsymbol{v}^{\prime}$ defined as follows:

$$
v_{i}^{\prime}:=v_{i} \cdot \prod_{j=1}^{l}\left(-\log \left|z_{\sigma(j)}\right|\right)^{-k_{j}\left(v_{i}\right)} .
$$

Recall that $Z(\sigma, l, C)$ denotes the following region:

$$
Z(\sigma, l, C):=\left\{\left.\left(z_{1}, \ldots, z_{n}\right) \in \Delta^{* n}|| z_{\sigma(i-1)}\right|^{C}<\left|z_{\sigma(i)}\right|, i=2, \ldots, l\right\} .
$$

Then we obtain the following theorem, which is the norm estimate in higher dimensional case.

Theorem 9.2. Let $C$ be a real number such that $C>1$. The frame $\boldsymbol{v}^{\prime}$ is adapted on $Z(\sigma, l, C)$. 
Proof. Clearly we only have to consider the case $\sigma=$ id. We can choose a natural number $N$ larger than $C$. And then, we only have to apply Theorem 9.1.

q.e.d.

Corollary 9.1. Let $f$ be a holomorphic section compatible with the sequence of the filtrations:

$$
\left(W\left(I_{1}\right), W\left(I_{2}\right), \ldots, W\left(I_{l}\right)\right)
$$

We put $2 \cdot k_{j}(f):=\operatorname{deg}^{W\left(I_{j}\right)}(f)-\operatorname{deg}^{W\left(I_{j-1}\right)}(f)$. Then we obtain the following estimate on $Z(\sigma, l, C)$ :

$$
0<C_{1}<|f|_{h} \cdot \prod_{j=1}^{l}\left(-\log \left|z_{\sigma(j)}\right|\right)^{-k_{j}(f)}<C_{2}
$$

Here $C_{i}(i=1,2)$ denote positive constants.

\subsubsection{Norm estimate for flat sections}

We give a brief argument to obtain a norm estimate for flat sections. For simplicity, we consider a tame nilpotent harmonic bundle $\left(E, \bar{\partial}_{E}, \theta, h\right)$ with trivial parabolic structure over $\Delta^{* n}$. Let $\left(\mathcal{E}^{1}, \mathbb{D}^{1}\right)$ denote the corresponding flat bundle, that is the deformed holomorphic bundle over $\{1\} \times \Delta^{* n}$.

Let $P$ be a point of $\Delta^{* n}$. Let $\gamma_{i}$ denote the loop defined as follows:

$$
z_{j}\left(\gamma_{i}(t)\right)= \begin{cases}z_{i}(P) \cdot \exp (2 \pi \sqrt{-1} t) & (j=i) \\ z_{j}(P) & (j \neq i)\end{cases}
$$

Then we obtain the monodromy $M\left(\gamma_{i}\right) \in \operatorname{End}\left(\mathcal{E}_{\mid P}^{1}\right)$. Since it is unipotent, we obtain the logarithm $N\left(\gamma_{i}\right) \in \operatorname{End}\left(\mathcal{E}_{\mid P}^{1}\right)$.

Let $\sigma$ be an element of $\mathfrak{S}_{n}$. Then we obtain the sets $I_{j}=\{\sigma(i) \mid i \leq$ $j\}$. We put $N\left(I_{j}\right):=\sum_{i \in I_{j}} N\left(\gamma_{i}\right)$. It induces the weight filtration $W\left(I_{j}\right)$.

Lemma 9.6. We have the following implication:

$$
\begin{cases}N\left(I_{j}\right)\left(W\left(I_{k}\right)_{h}\right) \subset W\left(I_{k}\right)_{h} & (k<j) \\ N\left(I_{j}\right)\left(W\left(I_{k}\right)_{h}\right) \subset W\left(I_{k}\right)_{h-2} & (k \geq j) .\end{cases}
$$


Proof. This is a consequence of Theorem 5.2 and Theorem 7.1. q.e.d.

We put $\mathbb{H}:=\{\zeta=x+\sqrt{-1} y \in \mathbf{C} \mid y>0\}$. Then we have the covering $\pi: \mathbb{H}^{n} \longrightarrow \Delta^{* n}$ given by $z_{i}=\exp \left(2 \pi \sqrt{-1} \zeta_{i}\right)$. We put as follows:

$$
\left.\begin{array}{rl}
\widetilde{Z}(\sigma, n, C, A):=\left\{\left(x_{1}+\sqrt{-1} y_{1}, \ldots,\right.\right. & \left.x_{n}+\sqrt{-1} y_{n}\right) \in \mathbb{H}^{n} \mid \\
\left|x_{i}\right|<A, & i=1, \ldots, n \\
C \cdot y_{i-1}>y_{i}, & i=2, \ldots, n
\end{array}\right\} .
$$

Take the pull back $\pi^{*}\left(\mathcal{E}^{1}, \mathbb{D}^{1}, h\right)$. Let $\widetilde{P}$ be a point of $\widetilde{Z}(\sigma, n, C, A)$ such that $\pi(\widetilde{P})=P$.

Take a nonzero element $v$ of $\mathcal{E}_{\mid P}^{1}$. We have the numbers $h_{j}:=$ $\operatorname{deg}^{W\left(I_{j}\right)}(v)$. We take a flat section $f$ such that $\pi_{*}\left(f_{\mid \widetilde{P}}\right)=v$. We have the following estimate of the norm of $f$.

Theorem 9.3. There exist positive numbers $C_{1}$ and $C_{2}$ such that the following equality holds on $\widetilde{Z}(\sigma, l, C, A)$ :

$$
0<C_{1} \leq|f|_{h}^{2} \cdot y_{1}^{h_{1}} \times \prod_{i=2}^{n} y_{i}^{h_{i}-h_{i-1}} \leq C_{2} .
$$

Proof. We give an only indication. We put as follows:

$$
g=\exp \left(\sum\left(\zeta_{i}-\zeta_{i}(\widetilde{P})\right) \cdot N\left(\gamma_{i}\right)\right) \cdot f
$$

Then $g$ is a holomorphic section of $\mathcal{E}^{1}$ defined over $\Delta^{*}$. In fact, it is a section of $\diamond \mathcal{E}^{1}$ compatible with the filtrations on the divisors. Thus we obtain the norm estimate for $g$.

By using Lemma 9.6, we can show that the logarithmic order of the norm of $g-f$ is lower than $f$. Thus we obtain the norm estimate of $f$, by using Corollary 9.1 .

q.e.d.

\subsection{Limiting CVHS}

\subsubsection{Taking a limit}

We put $X=\Delta^{n}, D_{i}=\left\{z_{i}=0\right\}$, and $D=\bigcup_{i=1}^{n} D_{i}$. For any nonnegative integer $m$, we have the morphism $\psi_{m, \underline{n}}: X \longrightarrow X$ defined as follows:

$$
\left(z_{1}, \ldots, z_{n}\right) \longmapsto\left(z_{1}^{m}, \ldots, z_{n}^{m}\right) .
$$


We will consider a limit of harmonic bundle via the pull backs of $\left\{\psi_{m n} \mid\right.$ $m \in \mathbb{Z}\}$.

Let $\left(E, \bar{\partial}_{E}, \theta, h\right)$ be a tame nilpotent harmonic bundle over $X-$ $D$. We have the deformed holomorphic bundle with the $\lambda$-connection by $(\mathcal{E}, \mathbb{D})$ and the prolongment ${ }^{\diamond} \mathcal{E}$. Let $\boldsymbol{v}$ be a holomorphic frame of ${ }^{\diamond} \mathcal{E}$, compatible with the filtration $W(\underline{n})$ on $\mathcal{D}_{\underline{n}}$. We put $2 \cdot k\left(v_{i}\right)=$ $\operatorname{deg}^{W(\underline{n})}\left(v_{i}\right)$.

Then we obtain the harmonic bundles $\psi_{m, n}^{*}\left(E, \bar{\partial}_{E}, \theta, h\right)$ on $X-D$. We also obtain the deformed holomorphic bundles and the $\lambda$-connections $\left(\psi_{m, \underline{n}}^{*} \diamond \mathcal{E}, \psi_{m, \underline{n}}^{*} \mathbb{D}\right)$. We obtain the holomorphic frame $\boldsymbol{v}^{(m)}$ of $\psi_{m, \underline{n}}^{*} \mathcal{E}$, defined as follows:

$$
v_{i}^{(m)}:=\psi_{m, \underline{n}}^{-1}\left(v_{i}\right) \cdot m^{-k\left(v_{i}\right)} .
$$

We put $H^{(m)}:=H\left(\psi_{m, \underline{n}}^{*}(h), \boldsymbol{v}^{(m)}\right)$, which is an $\mathcal{H}(r)$-valued function.

Lemma 9.7. Let $K$ be a compact subset of $\mathcal{X}-\mathcal{D}$. On $K$, the functions $H^{(m)}$ and $H^{(m)-1}$ are bounded independently of $m$. Moreover, we have the following estimate for sufficiently large $M$ and for a positive constant $C$, independently of $m$ :

$$
\left|v_{i}^{(m)}\right|_{\psi_{m}^{*}(h)} \leq C \cdot \prod_{i=1}^{n}\left(-\log \left|z_{i}\right|\right)^{M} .
$$

Proof. We only have to consider the boundedness on a compact subset $K \subset \mathbf{C}_{\lambda} \times Z(\sigma, n, N)$ when $\sigma=$ id $\in \mathfrak{S}_{n}$. Note that $Z(\mathrm{id}, n, N)$ is stable under $\psi_{m, \underline{n}}$.

Let $\boldsymbol{w}$ be a holomorphic frame of ${ }^{\diamond} \mathcal{E}$ over $\mathcal{X}$, which is compatible with the sequence of the filtrations $(N(\underline{1}), N(\underline{2}), \ldots, N(\underline{n}))$. We have the $C^{\infty}$-frame $\boldsymbol{w}^{\prime}$ over $\mathcal{X}-\mathcal{D}$, defined as follows:

$$
w_{i}^{\prime}:=w_{i} \cdot \prod_{j=1}^{n}\left(-\log \left|z_{j}\right|\right)^{-k_{j}\left(w_{i}\right)} .
$$

Here we put $2 \cdot k_{j}\left(w_{i}\right):=\operatorname{deg} W(\underline{j})\left(w_{i}\right)-\operatorname{deg}^{W} \underline{(j-1)}\left(w_{i}\right)$. As we have already seen, the frame $\boldsymbol{w}^{\prime}$ is adapted over $\Delta_{\lambda}(R) \times Z(\mathrm{id}, n, N)$, namely, $H(h, \boldsymbol{w})$ and $H(h, \boldsymbol{w})^{-1}$ are bounded over $\Delta_{\lambda}(R) \times Z(\mathrm{id}, n, N)$ for any $R>0$. It implies the boundedness of the functions $\left\{H\left(\psi_{m, \underline{n}}^{*} h, \psi_{m, \underline{n}} \boldsymbol{w}^{\prime}\right)\right\}$ and $\left\{H\left(\psi_{m, \underline{n}}^{*} h, \psi_{m, \underline{n}} \boldsymbol{w}^{\prime}\right)^{-1}\right\}$. 
We put $2 \cdot k\left(w_{i}\right)=\operatorname{deg}^{W(\underline{n})}\left(w_{i}\right)$. Then we have the following equality:

$$
\sum_{j=1}^{n} k_{j}\left(w_{i}\right)=k\left(w_{i}\right) .
$$

We have the following relation:

$$
\begin{aligned}
\psi_{m, \underline{n}}^{-1} w_{i}^{\prime} & =\psi_{m, \underline{n}}^{-1}\left(w_{i}\right) \cdot m^{-k\left(w_{i}\right)} \cdot \prod_{j=1}^{n}\left(-\log \left|z_{j}\right|\right)^{-k_{j}\left(w_{i}\right)} \\
& =w_{i}^{(m)} \cdot \prod_{j=1}^{n}\left(-\log \left|z_{j}\right|\right)^{-k_{j}\left(w_{i}\right)}
\end{aligned}
$$

Here we put $w_{i}^{(m)}:=\psi_{m, \underline{n}}^{-1}\left(w_{i}\right) \cdot n^{-k\left(w_{i}\right)}$. Let $L$ denote the diagonal matrix whose $(i, i)$-component is $\prod_{j=1}^{n}\left(-\log \left|z_{j}\right|\right)^{k_{j}\left(w_{i}\right)}$. Then we obtain the following relation:

$$
H\left(\psi_{m, \underline{n}}^{*} h, \boldsymbol{w}^{(m)}\right)=L \cdot H\left(\psi_{m, \underline{n}}^{*} h, \psi_{m, \underline{n}} \boldsymbol{w}^{\prime}\right) \cdot L .
$$

Thus we obtain the boundedness of $\left\{H\left(\psi_{m, n}^{*} h, \boldsymbol{w}^{(m)}\right)\right\}$ over a compact subset $K \subset \mathbf{C}_{\lambda} \times Z$ (id, $\left.n, N\right)$, independently of $m$. Similarly we obtain the boundedness of $\left\{H\left(\psi_{m, \underline{n}}^{*} h, \boldsymbol{w}^{(m)}\right)^{-1}\right\}$ over a compact subset $K \subset$ $\mathbf{C}_{\lambda} \times Z(\mathrm{id}, n, N)$, independently of $m$.

We have the relation $\boldsymbol{v}=\boldsymbol{w} \cdot B$, namely we have the following equalities:

$$
v_{j}=\sum_{i} b_{i j} \cdot w_{i}
$$

Note that $b_{i j}(\lambda, O)=0$ if $k\left(v_{j}\right)<k\left(w_{i}\right)$, for both of $\boldsymbol{v}$ and $\boldsymbol{w}$ are compatible with the filtration $W(\underline{n})$. We have the relation $\boldsymbol{v}^{(m)}=\boldsymbol{w}^{(m)}$. $B^{(m)}$. The component of $B^{(m)}$ is determined by the following:

$$
v_{j}^{(m)}=\sum_{i} \psi_{m, \underline{n}}^{*}\left(b_{i j}\right) \cdot m^{-k\left(v_{j}\right)+k\left(w_{i}\right)} \cdot w_{i}^{(m)}
$$

Namely we have $B_{i j}^{(m)}=\psi_{m, \underline{n}}^{*}\left(b_{i j}\right) \cdot m^{-k\left(v_{j}\right)+k\left(w_{i}\right)}$. It implies the boundedness of $B^{(m)}$ independently of $m$. Similarly we obtain the boundedness of $B^{(m)-1}$. Thus we obtain the boundedness of $H^{(m)}$ and $H^{(m)-1}$ over any compact subset $K$, independently of $m$. The estimates (63) are also obtained.

q.e.d.

Let us consider $\Omega(\boldsymbol{v})=v_{1} \wedge \cdots \wedge v_{r}$ and $\Omega\left(\boldsymbol{v}^{(m)}\right)=v_{1}^{(m)} \wedge \cdots \wedge v_{r}^{(m)}$. 
Lemma 9.8. Let $R$ be any positive number. There exist positive constants $C_{1}$ and $C_{2}$ satisfying the following inequality over $\{(\lambda, P) \in$ $\mathcal{X}-\mathcal{D}|| \lambda \mid<R\}$, for any $m$ :

$$
0<C_{1}<\left|\Omega\left(\boldsymbol{v}^{(m)}\right)\right|_{\psi_{m, \underline{n}}^{*}(h)}<C_{2} .
$$

Proof. Since $\Omega(\boldsymbol{v})$ gives a holomorphic frame of $\operatorname{det}\left({ }^{\diamond} \mathcal{E}\right)$, we have the following inequality for some $0<C_{1}<C_{2}$ :

$$
0<C_{1}<|\Omega(\boldsymbol{v})|_{h}<C_{2} .
$$

Then it is clear we obtain the result.

q.e.d.

We put $U_{h}:=\left\langle v_{i} \mid \operatorname{deg}^{W(\underline{n})}\left(v_{i}\right)=h\right\rangle$. We have the $\lambda$-connection form $\mathcal{A}$ of $\mathbb{D}$ with respect to the frame $\boldsymbol{v}$. We decompose $\mathcal{A}$ into $\sum_{j} \mathcal{A}_{j}=$ $\sum A_{j} \cdot d z_{j} / z_{j}$. We also decompose $A_{j}$ into $A_{j(h, k)}$ corresponding to the decomposition ${ }^{\diamond} \mathcal{E}=\bigoplus U_{h}$ (see Subsubsection 6.2.2). By our choice of $U_{h}$, we have the vanishing $A_{j(h, k)}(\lambda, O)=0$ when $h>k-1$.

Let $\mathcal{A}^{(m)}=\sum \mathcal{A}_{j}^{(m)}$ denote the $\lambda$-connection form of $\psi_{m, \underline{n}}^{-1} \mathbb{D}$ with respect to the frame $\boldsymbol{v}^{(m)}$.

Lemma 9.9. We have the following equalities:

$$
\mathcal{A}_{j}^{(m)}=\sum_{h, k} \psi_{m, \underline{n}}^{*} A_{j(h, k)} \cdot m^{(h-k+2) / 2} \cdot \frac{d z_{j}}{z_{j}} .
$$

In particular, the sequences $\left\{\mathcal{A}^{(m)}\right\}$ converges to $\mathcal{A}^{(\infty)}=\sum_{j} \mathcal{A}_{j}^{(\infty)}$ given as follows:

$$
\mathcal{A}_{j}^{(\infty)}=\sum_{h} A_{j(h, h+2)}(\lambda, O) \cdot \frac{d z_{j}}{z_{j}} .
$$

Proof. Similar to Lemma 6.9.

q.e.d.

We decompose $\theta$ into $\sum f_{j} \cdot d z_{j} / z_{j}$. Then $\psi_{m}^{*} \theta=\sum_{j} m \cdot \psi_{m, \underline{n}}^{-1}\left(f_{j}\right) \cdot$ $d z_{j} / z_{j}$. Since we have the estimate $\left|f_{j}\right|_{h} \leq C \cdot\left(-\log \left|z_{j}\right|\right)^{-1}$, we obtain the following estimate independently of $m$ :

$$
\left|m \cdot \psi_{m, \underline{n}}^{*}\left(f_{j}\right)\right|_{\psi_{m}^{*}(h)} \leq C \cdot\left(-\log \left|z_{j}\right|\right)^{-1} .
$$

In all we obtain the following.

Lemma 9.10. The sequence $\left\{\boldsymbol{v}^{(m)}\right\}$ satisfies Condition 3.2. 
We can apply the result in Subsection 3.3, as in Subsubsection 6.2.3. Then we can pick a subsequence $\left\{m_{i}\right\}$ of $\{m\}$ and the limiting harmonic bundle $\left(F, \theta^{(\infty)}, h^{(\infty)}\right)$ for $\left\{m_{i}\right\}$. We also obtain the deformed holomorphic bundle with $\lambda$-connection $\left(\mathcal{F}^{(\infty)}, \mathbb{D}^{(\infty)}\right)$ and the holomorphic frame $\boldsymbol{v}^{(\infty)}$. Due to the estimates (63), we obtain the estimates $\left|v_{i}^{(\infty)}\right|_{h^{(\infty)}}<C \cdot \prod_{j=1}^{n}\left(-\log \left|z_{j}\right|\right)^{M}$ for sufficiently large $M$. We also obtain the estimate $0<C_{1}<\left|\Omega\left(\boldsymbol{v}^{(\infty)}\right)\right|_{h^{(\infty)}}$. Thus $\boldsymbol{v}^{(\infty)}$ naturally gives a holomorphic frame of the prolongment ${ }^{\diamond} \mathcal{F}^{(\infty)}$.

We have the decomposition $\mathcal{F}^{(\infty)}=\bigoplus_{h} \mathcal{U}_{h}$, where $\mathcal{U}_{h}$ denotes the vector subbundle generated by $\left\{v_{i}^{(\infty)} \mid \operatorname{deg}{ }^{W(\underline{n})} v_{i}=h\right\}$.

Lemma 9.11. The subbundles $\mathcal{U}_{h}$ are independent of a choice of the original frame compatible with $W(\underline{n})$.

Proof. Similar to Lemma 6.14. q.e.d.

We have the $\lambda$-connection form $\mathcal{A}^{(\infty)}=\sum A_{j}^{(\infty)} d z_{j} / z_{j}$ of $\mathbb{D}^{(\infty)}$ with respect to the frame $\boldsymbol{v}^{(\infty)}$. Let $f_{A_{j}^{(\infty)}}$ denote the endomorphism determined by $A_{j}^{(\infty)}$ and $\boldsymbol{v}^{(\infty)}$.

Lemma 9.12. We have $f_{A_{j}^{(\infty)}}\left(\mathcal{U}_{h}\right) \subset \mathcal{U}_{h-2}$. In particular, we obtain $\theta\left(\mathcal{U}_{h}\right) \subset \mathcal{U}_{h-2} \otimes \Omega_{X-D}^{1,0}$.

Proof. The claim immediately follows from Lemma 9.9. $\quad$ q.e.d.

We have the $\mathcal{H}(r)$-valued function $H\left(h^{(\infty)}, v^{(\infty)}\right)$. We use the real coordinate $z_{i}=r_{i} \cdot \exp \left(2 \pi \sqrt{-1} \alpha_{i}\right)$ for $i=1, \ldots, n$.

Lemma 9.13. The function $H\left(h^{(\infty)}, v^{(\infty)}\right)$ is independent of $\alpha_{i}$ for any $i$.

Proof. Similar to Proposition 6.2.

q.e.d.

We put $\mathcal{U}^{\lambda}:=\mathcal{U}_{\mid \mathcal{X}^{\lambda}-\mathcal{D}^{\lambda}}$.

Theorem 9.4. If $h \neq h^{\prime}$, then $\mathcal{U}_{h}^{0}$ and $\mathcal{U}_{h^{\prime}}^{0}$ are orthogonal.

Proof. We put $\Delta_{\mathbf{R},+}^{*, n}=\mathbf{R}_{>0}^{n} \cap \Delta^{* n}$. We have the following:

$$
\begin{aligned}
& H\left(h^{(\infty)}, \boldsymbol{v}^{(\infty)}\right)\left(r_{1} \cdot \exp \left(2 \pi \sqrt{-1} \alpha_{1}\right), \ldots r_{n} \cdot \exp \left(2 \pi \sqrt{-1} \alpha_{n}\right)\right) \\
& =H\left(h^{(\infty)}, \boldsymbol{v}^{(\infty)}\right)\left(r_{1} \ldots r_{n}\right) .
\end{aligned}
$$

Thus we only have to check the orthogonality over $\Delta_{\mathbf{R},+}^{* n}$.

Let $\left(r_{1}, \ldots, r_{n}\right)$ be an element of $\Delta_{\mathbf{R},+}^{* n}$. Then we have the real numbers $\alpha_{j}>0$ satisfying $r_{j}=r_{1}^{\alpha_{j}}$. The real numbers $\alpha_{j}$ can be 
approximated by rational numbers. For any $\boldsymbol{h} \in \mathbb{Z}_{>0}^{n}$, we put $C_{\boldsymbol{h}, \mathbf{R},+}:=$ $\left\{\left(t^{h_{1}}, \ldots, t^{h_{n}}\right) \mid 0<t<1\right\} \subset \Delta_{\mathbf{R},+}^{* n}$. Then we know the following set is dense in $\Delta_{\mathbf{R},+}^{* n}$ :

$$
\bigcup_{h \in \mathbb{Z}_{>0}^{n}} C_{h, \mathbf{R},+} .
$$

Thus we only have to check the orthogonality on $C_{\boldsymbol{h}, \mathbf{R},+}$.

We put $C_{\boldsymbol{h}}:=\left\{\left(t_{1}^{h_{1}}, \ldots, t_{n}^{h_{n}}\right) \in \Delta^{*}\right\}$. Since we have $C_{\boldsymbol{h}, \mathbf{R},+} \subset C_{\boldsymbol{h}}$, we only have to check the orthogonality on $C_{\boldsymbol{h}}$. Note that $C_{\boldsymbol{h}}$ is the image of the morphism $g_{h}: \Delta^{*} \longrightarrow X-D$, and that we have the following commutative diagram:

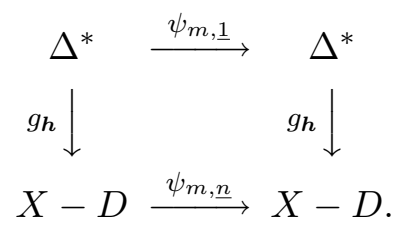

Thus Theorem 9.4 is reduced to Theorem 6.1.

q.e.d.

As a direct corollary, we obtain the following theorem.

Theorem 9.5. The tuple $\left(F, \theta^{(\infty)}, h^{(\infty)}\right)$ gives a complex variation of polarized Hodge structure, up to grading.

Proof. Similar to Corollary 6.5.

q.e.d.

Definition 9.1. The tuple $\left(F, \theta^{(\infty)}, h^{(\infty)}\right)$ is called a limiting CVHS of $(E, \theta, h)$.

\subsubsection{Real structure}

Let us consider the real structure of the harmonic bundles. Let $\left(E, \bar{\partial}_{E}, \theta, h\right)$ be a harmonic bundle over a complex manifold $X$.

Definition 9.2. Let $\iota: E \longrightarrow E$ be an anti-linear isomorphism. We say that $\iota$ is a real structure of $\left(E, \bar{\partial}_{E}, \theta, h\right)$, if the following holds:

- $\iota^{2}$ is the identity map.

- $\iota$ preserves the metric $h$.

- $\iota$ replaces $\partial_{E}$ and $\bar{\partial}_{E}$. Namely we have $\iota\left(\partial_{E} f\right)=\bar{\partial}_{E} \iota(f)$ and $\iota\left(\bar{\partial}_{E} f\right)=\partial_{E} \iota(f)$. 
- $\iota$ replaces $\theta$ and $\theta^{\dagger}$.

By the natural anti-linear isomorphism $E \simeq E^{\vee}$ induced by the hermitian metric $h$, we can also regard $\iota$ as the holomorphic isomorphism $E \longrightarrow E^{\vee}$.

Remark 9.1. Recall that the harmonic bundle is regarded as a variation of pure polarized twistor structure, according to Simpson ([36]). Although the real structure of harmonic bundle should be seen from such view point, we do not discuss such issue.

We put $X=\Delta^{n}, D_{i}=\left\{z_{i}=1\right\}$ and $D=\bigcup_{i=1}^{n} D_{i}$. Let $\left(E, \bar{\partial}_{E}, \theta, h\right)$ be a tame nilpotent harmonic bundle with trivial parabolic structure over $X-D$. Assume that $\left(E, \bar{\partial}_{E}, \theta, h\right)$ has a real structure $\iota$. Consider the sequence $\left\{\psi_{m, \underline{n}}^{*}\left(E, \bar{\partial}_{E}, \theta, h\right)\right\}$ in Subsubsection 9.2.1. As in 6.2.3, Let $F=\bigoplus_{i=1}^{r} \mathcal{O}_{X-D} \cdot u_{i}$ be a holomorphic bundle with the frame $\boldsymbol{u}=$ $\left(u_{i}\right)$, over $X-D$. We put $\boldsymbol{e}^{(m)}:=\boldsymbol{v}_{\mid \mathcal{X}^{0}}^{(m)}$. It is the frame of $\diamond_{m, \underline{1}}^{*} \mathcal{E}^{0}$ over $\mathcal{X}^{0}=\{0\} \times X$. The frames $\boldsymbol{e}^{(m)}$ and $\boldsymbol{u}$ give the holomorphic isomorphism $\Phi_{m}: \mathcal{E}^{0} \longrightarrow F$ over $X-D$. The morphism $\Phi_{m}$ induces the structure of harmonic bundle on $F$. Moreover we have the sequence of real structure $\iota^{(m)}$, which is the image of $\psi_{m, \underline{n}}^{*}(\iota)$ via the morphism $\Phi_{m}$.

Note that $\left|\psi_{m, \underline{n}}(\iota)\right|_{\psi_{m, \underline{n}}^{*}(h)}=1$, and they are holomorphic as morphisms $\psi_{m, \underline{n}}^{*} E \longrightarrow \psi_{m, \underline{n}}^{*} E^{\vee}$. Thus we can pick a subsequence $\left\{m_{i}\right\}$ for a limiting CVHS $\left(F, \theta^{(\infty)}, h^{(\infty)}\right)$ such that the sequence $\left\{\iota^{\left(m_{i}\right)}\right\}$ also converges. We denote the limit by $\iota^{(\infty)}$.

Lemma 9.14. $\iota^{(\infty)}$ gives a real structure to the limiting CVHS $\left(F, \theta^{(\infty)}, h^{(\infty)}\right)$.

Proof. It is clear from our construction that the conditions in 9.2 are satisfied. Thus $\iota^{(\infty)}$ gives a real structure of a harmonic bundle.

Thus we only have to check $\iota^{(\infty)}\left(\mathcal{U}_{h}^{0}\right)=\mathcal{U}_{-h}^{0}$. Consider the filtration $\mathcal{W}$ and $\mathcal{W}^{\dagger}$ given as follows:

$$
\mathcal{W}_{h}:=\bigoplus_{l \leq h} \mathcal{U}_{l}^{0}, \quad \mathcal{W}_{h}^{\dagger}:=\bigoplus_{l \geq-h} \mathcal{U}_{l}^{0}
$$

From $\iota\left(\theta^{(\infty)}\right)=\theta^{(\infty) \dagger}$, it is easy to see $\iota\left(\mathcal{W}_{h}\right)=\mathcal{W}_{-h}^{\dagger}$. Then we obtain $\iota\left(\mathcal{U}_{h}^{0}\right)=\mathcal{U}_{-h}^{0}$, by using the orthogonality of them and an easy ascending induction on $h$.

q.e.d.

Definition 9.3. The tuple $\left(F, \theta^{(\infty)}, h^{(\infty)}, \iota^{(\infty)}\right)$ is called a limiting RVHS. 


\subsubsection{Purity theorem}

Recall the setting of the purity theorem, following Kashiwara and Kawai (See [24] and [25]. But our filtration is slightly different from theirs. See remark 9.2.) Let $V$ be a finite dimensional vector space and $\left(N_{1}, \ldots, N_{n}\right)$ be a commuting tuple of nilpotent maps. We put $N(\underline{j})=\sum_{i \leq j} N_{i}$. We denote the weight filtration of $N(\underline{n})$ by $W(\underline{n})$. Let $\left(e_{1}, \ldots, e_{n}\right)$ be the standard base of $\mathbb{Z}^{n}$. We have the partial Koszul complex $\Pi\left(N_{1}, \ldots, N_{n}\right)$. The $k$-th part $\Pi\left(N_{1}, \ldots, N_{n}\right)^{k}$ is defined as follows:

$$
\Pi\left(N_{1}, \ldots, N_{n}\right)^{k}=\bigoplus_{\substack{|J|=k, J \subset \underline{n}}} \operatorname{Im} N_{J} \otimes\left(\mathbf{C} \cdot e_{J}\right) .
$$

Here we put $N_{J}=\prod_{j \in J} N_{j}$ and $e_{J}=\bigwedge_{j \in J} e_{j}$. The differential $d$ is given by $\sum_{j=1}^{n} N_{j} \wedge e_{j}$. Namely we put as follows:

$$
d\left(v \otimes e_{J}\right):=\sum_{j=1}^{n} N_{j}(v) \otimes\left(e_{j} \wedge e_{J}\right) .
$$

The filtration $W$ of $\operatorname{Im}\left(N_{j}\right)$ is given as follows:

$$
W_{h}\left(\operatorname{Im}\left(N_{J}\right)\right)=N_{J}\left(W_{h}(\underline{n})\right) \subset \operatorname{Im}\left(N_{J}\right) .
$$

Then we obtain the filtration $W$ of $\Pi\left(N_{1}, \ldots, N_{n}\right)^{k}$ as follows:

$$
W_{h}\left(\Pi\left(N_{1}, \ldots, N_{n}\right)^{k}\right):=\bigoplus_{\substack{J \mid=k, J \subset \underline{n} \\ \subset \subset \underline{n}}} W_{h}\left(\operatorname{Im}\left(N_{J}\right)\right) \otimes\left(\mathbf{C} \cdot e_{J}\right) .
$$

We put $X=\Delta^{n}, D_{i}=\left\{z_{i}=0\right\}$ and $D=\bigcup_{i=1}^{n} D_{i}$. Let $\left(E, \bar{\partial}_{E}, \theta, h\right)$ be a tame nilpotent harmonic bundle with trivial parabolic structure over $X-D$. We put $V={ }^{\diamond} \mathcal{E}_{\mid(\lambda, O)}$. Then we have the nilpotent maps $N_{i}:=\operatorname{Res}_{\mathcal{D}_{i}}(\mathbb{D})_{\mid(\lambda, O)}$ for $i=1, \ldots, n$.

Lemma 9.15. In this case, we have the following:

$$
W_{k}\left(\operatorname{Im}\left(N_{J}\right)\right)=\operatorname{Im}\left(N_{J}\right) \cap W_{k-2|J|} .
$$

Proof. Take a point $P \in X-D$, and then the filtration $W^{\triangle}(\underline{n})$ gives a mixed twistor structure to the vector bundle $S(O, P)$. Then Lemma 9.15 is a consequence of Lemma 2.20. q.e.d.

We have the induced filtration on the cohomology group $H^{*}\left(\Pi\left(N_{1}\right.\right.$, $\left.\left.\ldots, N_{n}\right)\right)$. 
Theorem 9.6. Assume that $\left(E, \bar{\partial}_{E}, \theta, h\right)$ has a real structure. Then the purity theorem holds for the tuple $\left(V, N_{1}, \ldots, N_{n}\right)$. Namely we have the following:

$$
H^{k}\left(\Pi\left(N_{1}, \ldots, N_{n}\right)\right)=W_{k}\left(H^{k}\left(\Pi\left(N_{1}, \ldots, N_{n}\right)\right)\right) .
$$

In other words, the following morphism is surjective:

$$
W_{k}\left(\Pi\left(N_{1}, \ldots, N_{n}\right)\right)^{k} \cap \operatorname{Ker}(d) \longrightarrow H^{k}\left(\Pi\left(N_{1}, \ldots, N_{n}\right)\right) .
$$

Proof. Let $G r .\left(\Pi\left(N_{1}, \ldots, N_{n}\right)\right)$ denote the associated graded complex of the filtered complex $\Pi\left(N_{1}, \ldots, N_{n}\right)$. Then we obtain the following natural isomorphism:

$$
\begin{aligned}
G r_{h}\left(\Pi\left(N_{1}, \ldots, N_{n}\right)\right)^{a}: & =\bigoplus_{\substack{|J|=a, J \subset \underline{n}}} \frac{N_{J}\left(W_{h}\right)}{N_{J}\left(W_{h-1}\right)} \otimes\left(\mathbf{C} \cdot e_{J}\right) \\
& \simeq \bigoplus_{\substack{|J|=a, J \subset \underline{n}}} \frac{N_{J}\left(W_{h}\right)}{N_{J}\left(W_{h}\right) \cap W_{h-1-2 a}} \otimes\left(\mathbf{C} \cdot e_{J}\right) .
\end{aligned}
$$

Lemma 9.16. To show Theorem 9.6, we only have to show the following:

$$
H^{a}\left(G r_{k}\left(\Pi\left(N_{1}, \ldots, N_{n}\right)\right)\right)=0, \quad \text { if } a<k .
$$

Proof. It is shown by an elementary homological algebraic argument. q.e.d.

We put $V_{h}^{(\infty)}=G r_{h}^{W(\underline{n})}$, and $V^{(\infty)}=\bigoplus_{h} V_{h}^{(\infty)}$. The morphism $N_{i}$ induces $N_{i h}^{(\infty)}: V_{h}^{(\infty)} \longrightarrow V_{h-2}^{(\infty)}$ for any $h$. We denote the direct sum $\bigoplus_{h} N_{i h}^{(\infty)}$ by $N_{i}^{(\infty)}$. Then we obtain the commuting tuple $\left(N_{1}^{(\infty)}, \ldots, N_{n}^{(\infty)}\right)$.

Consider a limiting RVHS $\left(F, \theta^{(\infty)}, h^{(\infty)}\right)$ of $(E, \theta, h)$ obtained in Subsubsection 9.2.1. Let $\mathcal{F}^{(\infty)}$ denote the deformed holomorphic bundle. Due to Lemma 9.9 and the fact that $\boldsymbol{v}^{(\infty)}$ gives a frame of $\diamond \mathcal{F}^{(\infty)}$, the tuple $\left(V^{(\infty)}, N_{1}^{(\infty)}, \ldots, N_{n}^{(\infty)}\right)$ is isomorphic to the following tuple:

$$
\left({ }^{\diamond} \mathcal{F}^{(\infty)}{ }_{\mid(\lambda, O)}, \operatorname{Res}_{\mathcal{D}_{1}}\left(\mathbb{D}^{(\infty)}\right)_{\mid(\lambda, O)}, \ldots, \operatorname{Res}_{\mathcal{D}_{n}}\left(\mathbb{D}^{(\infty)}\right)_{\mid(\lambda, O)}\right) .
$$

For a RVHS, the purity theorem holds, due to the result of CattaniKaplan-Schmid [7] or Kashiwara-Kawai [25]. 
Note that the complex $\Pi\left(N_{1}^{(\infty)}, \ldots, N_{n}^{(\infty)}\right)$ is graded. The grading is induced by the grading of $V^{(\infty)}$. Namely we have the following decomposition:

$$
\Pi\left(N_{1}^{(\infty)}, \ldots, N_{n}^{(\infty)}\right)^{a}=\bigoplus_{h}\left(\bigoplus_{|J|=a, J \subset \underline{n}} N_{J}^{(\infty)}\left(V_{h}^{(\infty)}\right)\right) .
$$

It is easy to see that the differential preserves the grading. Thus we obtain the following complex:

$$
\Pi\left(N_{1}^{(\infty)}, \ldots, N_{n}^{(\infty)}\right)_{h}^{a}=\bigoplus_{|J|=a, J \subset \underline{n}} N_{J}^{(\infty)}\left(V_{h}^{(\infty)}\right), \quad d=\sum_{j=1}^{n} N_{j}^{(\infty)} \wedge e_{j} .
$$

Since the decomposition of $V^{(\infty)}$ gives the splitting of the filtration of $V^{(\infty)}$, the decomposition (64) gives the splitting of the filtration of $\Pi\left(N_{1}^{(\infty)}, \ldots, N_{n}^{(\infty)}\right)$. Namely we have the following:

$$
W_{k}\left(\Pi\left(N_{1}^{(\infty)}, \ldots, N_{n}^{(\infty)}\right)\right)^{a}=\bigoplus_{h \leq k} \Pi\left(N_{1}^{(\infty)}, \ldots, N_{n}^{(\infty)}\right)_{h}^{a}
$$

Then the claim of the purity theorem for the tuple $\left(V^{(\infty)}, N_{1}^{(\infty)}, \ldots\right.$, $N_{n}^{(\infty)}$ ) implies the following vanishing:

$$
H^{a}\left(\Pi\left(N_{1}^{(\infty)}, \ldots, N_{n}^{(\infty)}\right)_{k}\right)=0, \quad \text { if } a<k .
$$

By our construction, we have the following:

$$
\Pi\left(N_{1}^{(\infty)}, \ldots, N_{n}^{(\infty)}\right)_{k}^{a} \simeq \bigoplus_{|J|=a, J \subset \underline{n}} \frac{N_{J}\left(W_{k}\right)}{N_{J}\left(W_{k}\right) \cap W_{k-1-2 a}} .
$$

Namely we have the isomorphism of the complexes:

$$
G r_{k}\left(\Pi\left(N_{1}, \ldots, N_{n}\right)\right) \simeq \Pi\left(N_{1}^{(\infty)}, \ldots, N_{n}^{(\infty)}\right)_{k} .
$$

Due to (65), (66) and Lemma 9.16, we obtain Theorem 9.6. q.e.d.

Remark 9.2. We should remark the relations between the weight filtration $W$ of $\Pi\left(N_{1}, \ldots, N_{n}\right)$ considered here, $W^{\text {cks }}$ given by CattaniKaplan-Schmid ([7]), and $W^{k k}$ given by Kashiwara-Kawai ([25]). The weight filtration $W^{c k s}$ of Cattani-Kaplan-Schmid is as follows:

$$
W_{h}^{c k s}\left(\Pi\left(N_{1}, \ldots, N_{n}\right)^{a}\right)=W_{a+h}\left(\Pi\left(N_{1}, \ldots, N_{n}\right)^{a}\right) .
$$


The weight filtration $W^{k k}$ of $\Pi\left(N_{1}, \ldots, N_{n}\right)^{a}$ for the RVHS of weight $w$ is given as follows:

$$
W_{h}^{k k}\left(\Pi\left(N_{1}, \ldots, N_{n}\right)^{a}\right)=W_{h-w}\left(\Pi\left(N_{1}, \ldots, N_{n}\right)^{a}\right) .
$$

The results can be stated as $W_{0}^{c k s} H^{h}=W_{h+w}^{k k} H^{h}=W_{h} H^{h}=H^{h}$.

Remark 9.3. We should remark that we do not obtain another proof of the purity theorem for RVHS. We just reduced the purity theorem for tame nilpotent harmonic bundles with trivial parabolic structure to the purity theorem for RVHS.

\section{Appendix}

We recall the definition of complex variation of polarized Hodge structure (CVHS), and the relation of CVHS with a harmonic bundle. We also recall the real variation of polarized Hodge structure (RVHS).

Let $X$ be a complex manifold. Recall the definition of complex variation of polarized Hodge structures (See Section 8 of [34], for example).

Definition 10.1. Let $V$ be a $C^{\infty}$-vector bundle over $X$ with a decomposition $V=\bigoplus_{p+q=w} V^{p, q}$. Let $\mathbb{D}^{1}$ be a flat connection of $V$, and $\langle\cdot, \cdot\rangle$ be a sesqui-linear form on $V$. The tuple $\left(\bigoplus_{p+q=w} V^{p, q}, \mathbb{D}^{1},\langle\cdot, \cdot\rangle\right)$ is called a complex variation of Hodge structure of weight $w$, if the following conditions are satisfied:

- We have the following implication:

$$
\begin{gathered}
\mathbb{D}^{1}\left(C^{\infty}\left(X, V^{p, q}\right)\right) \subset C^{\infty}\left(X, V^{p+1, q-1} \otimes \Omega^{0,1}\right) \\
\oplus C^{\infty}\left(X, V^{p, q} \otimes \Omega^{0,1}\right) \\
\oplus C^{\infty}\left(X, V^{p, q} \otimes \Omega^{1,0}\right) \oplus C^{\infty}\left(X, V^{p-1, q+1} \otimes \Omega^{1,0}\right) .
\end{gathered}
$$

- $\langle u, v\rangle=(-1)^{w} \overline{\langle v, u\rangle}$.

- $V^{p, q}$ and $V^{p^{\prime}, q^{\prime}}$ are orthogonal if $(p, q)$ and $\left(p^{\prime}, q^{\prime}\right)$ are different.

- We denote the restriction of $\langle\cdot, \cdot\rangle$ by $\langle\cdot, \cdot\rangle_{(p, q)}$.

Then $(\sqrt{-1})^{p-q}\langle\cdot, \cdot\rangle_{(p, q)}$ is positive definite on $V^{p, q}$. 
A complex variation of Hodge structure is called CVHS in this paper, for simplicity.

Let $\left(\bigoplus_{p+q=w} V^{p, q}, \mathbb{D}^{1},\langle\cdot, \cdot\rangle\right)$ be a CVHS on $X$. We obtain the metric $h$ given as follows:

$$
h=\bigoplus_{p+q=w}(\sqrt{-1})^{p-q}\langle\cdot, \cdot\rangle_{(p, q)} .
$$

We have the decomposition of $\mathbb{D}^{1}$ corresponding to the decomposition $V \otimes \Omega^{1}=\bigoplus V^{p, q} \otimes\left(\Omega^{0,1} \oplus \Omega^{1,0}\right):$

$$
\mathbb{D}^{1}=\theta^{\dagger}+\bar{\partial}_{V}+\partial_{V}+\theta
$$

The following proposition can be shown by a direct calculation.

Proposition 10.1. A tuple $\left(V, \bar{\partial}_{V}, \theta, h\right)$ is a harmonic bundle. The $(1,0)$-part of the unitary metric associated with $\bar{\partial}_{V}$ and $h$ is given by $\partial_{V}$, and the adjoint of $\theta$ is $\theta^{\dagger}$.

On the other hand, we obtain a CVHS from a harmonic bundle with a nice grading. Consider the harmonic bundle $\left(E, \bar{\partial}_{E}, \theta, h\right)$, with a holomorphic decomposition $E=\bigoplus_{l} E_{l}$. Assume that the following:

- $\theta\left(E_{l}\right) \subset E_{l-1} \otimes \Omega^{1,0}$.

- If $l \neq l^{\prime}$, then $E_{l}$ and $E_{l^{\prime}}$ are orthogonal with respect to the metric $h$.

Let $w$ be an integer. For example, we can put $V^{p, q}=E_{p}$ for a pair $(p, q)$ such that $p+q=w$. We have the flat connection $\mathbb{D}^{1}$ of $C^{\infty}$-bundle $V=\bigoplus V^{p, q}=E$. By reversing the construction above, we obtain the flat sesqui linear form $\langle\cdot, \cdot\rangle$ on $V$. Then the tuple $\left(\bigoplus V^{p, q}, \mathbb{D}^{1},\langle\cdot, \cdot\rangle\right)$ gives a CVHS. We do not have to care a choice of the weight $w$ and the way of superscripts $(p, q)$.

Consider the harmonic bundle $\left(E, \bar{\partial}_{E}, \theta, h\right)$, with a holomorphic decomposition $E=\bigoplus_{l} E_{l}$. Assume that the following:

- $\theta\left(E_{l}\right) \subset E_{l-2} \otimes \Omega^{1,0}$.

- If $l \neq l^{\prime}$, then $E_{l}$ and $E_{l^{\prime}}$ are orthogonal with respect to the metric $h$. 
In this case, we can put, for example, as follows: For a pair $(p, q) \in \mathbb{Z}^{2}$ such that $p+q=0$, we put $V_{0}^{p, q}=E_{p-q}$. For a pair $(p, q) \in \mathbb{Z}^{2}$ such that $p+q=1$, we put $V_{1}^{p, q}=E_{p-q}$. We put $V_{0}=\bigoplus_{p+q=0} V^{p, q}$ and $V_{1}=\bigoplus_{p+q=1} V^{p, q}$. Then $E$ is decomposed into $V_{0} \oplus V_{1}$. We have the induced structures of harmonic bundles on $V_{0}$ and $V_{1}$. They are complex variations of polarized Hodge structures weight 0 and 1 respectively.

Definition 10.2. Let $\left(\bigoplus_{p+q=w} V^{p, q}, \mathbb{D}^{1},\langle\cdot, \cdot\rangle\right)$ be a CVHS on $X$. A real structure $\iota$ of $\left(\bigoplus_{p+q=w} V^{p, q}, \mathbb{D}^{1},\langle\cdot, \cdot\rangle\right)$ is an anti-linear $C^{\infty}$ isomorphism $V \longrightarrow V$ satisfying the next conditions:

- $\iota^{2}=\mathrm{id}_{V}$ and $\iota\left(V^{p, q}\right)=V^{q, p}$.

- $\iota$ preserves the metric $\bigoplus_{p+q=w}(-\sqrt{-1})^{p-q}\langle\cdot, \cdot\rangle$.

- $\iota$ is flat with respect to $D$. Equivalently, $\iota$ replaces $\left(\bar{\partial}_{V}, \theta^{\dagger}\right)$ and $\left(\partial_{V}, \theta\right)$.

Such a tuple $\left(\bigoplus_{p+q=w} V^{p, q}, \mathbb{D}^{1},\langle\cdot, \cdot\rangle, \iota\right)$ is called a real variation of polarized Hodge structures. For simplicity, it is called RVHS in this paper.

\section{Note Added in Proof.}

Some theorems are strengthened easily. We only mention the following. The details will be discussed elsewhere.

1. We considered the filtered vector bundle $(S(O, P), W)$. (See Subsection 5.2.) It can be shown that $\mathrm{Gr}_{i}^{W} S(O, P)$ are independent of a choice of the points $P$ in $X-D$. Hence Theorem 5.1 can be strengthened as follows: The filtered vector bundle is a mixed twistor for any point $P \in \Delta^{*}$.

2. In Theorem 9.6, we do not have to impose the real structure. In fact, the purity theorem for $\left(E, \bar{\partial}_{E}, \theta, h\right)$ is reduced to the purity theorem for $\left(E, \bar{\partial}_{E}, \theta, h\right) \oplus\left(E^{\vee}, \bar{\partial}_{E^{\vee}}, h^{\vee}, \theta^{\vee}\right)$. The latter has the canonical real structure.

\section{References}

[1] L.V. Ahlfors, An extension of Schwarz's lemma, Trans. Amer. Math. Soc. 43 (1938) 359-364, MR 1501 949, Zbl 0018.41002. 
[2] A. Andreotti \& E. Vesentini, Carleman estimates for the Laplace-Beltrami equation on complex manifolds, Inst. Hautes Etudes Sci. Publ. Math. 25 (1965) 313-362, MR 30 \#5333, Zbl 0138.06604.

[3] T. Aubin, Nonlinear analysis on manifolds. Monge-Ampére equations, Grundlehren der Mathematischen Wissenschaften 252, Springer-Verlag, New York, 1982, MR 85j:58002, Zbl 0512.53044.

[4] O. Biquard, Fibrés de Higgs et connexions intégrables: le cas logarithmique (diviseur lisse), Ann. Sci. École Norm. Sup. 30 (1997) 41-96, MR 98e:32054, Zbl 0876.53043.

[5] E. Cattani \& A. Kaplan, Polarized mixed Hodge structures and the local monodromy of a variation of Hodge structure, Invent. Math. 67 (1982) 101-115, MR 84a:32046, Zbl 0516.14005.

[6] E. Cattani, A. Kaplan \& W. Schmid, Degeneration of Hodge structures, Ann. of Math. 123 (1986) 457-535, MR 88a:32029, Zbl 0617.14005.

[7] E. Cattani, A. Kaplan \& W. Schmid, $L^{2}$ and intersection cohomologies for a polarized variation of Hodge structure, Invent. Math. 87 (1987) 217-252, MR 88h:32019, Zbl 0611.14006.

[8] E. Cattani, A. Kaplan \& W. Schmid, Variations of polarized Hodge structure: asymptotics and monodromy, Lecture Notes in Math., 1246, Springer, Berlin, 1987, 16-31, MR 88h:32020, Zbl 0626.14008.

[9] E. Cattani, A. Kaplan \& W. Schmid, Some remarks on $L^{2}$ and intersection cohomologies, Lect. Notes in Math., 1246, Springer, Berlin, 1987, 32-41, MR 89f:32018, Zbl 0624.14013.

[10] K. Corlette, Flat G-bundles with canonical metrics, J. Differential Geom. 28 (1988) 361-382, MR 89k:58066, Zbl 0676.58007.

[11] M. Cornalba \& P. Griffiths, Analytic cycles and vector bundles on noncompact algebraic varieties, Invent. Math. 28 (1975) 1-106, MR 51 \#3505, Zbl 0293.32026.

[12] P. Deligne, Equation differentielles a points singularier reguliers, Lectures Notes in Maths., 163, Springer, 1970, MR 54 \#5232, Zbl 0244.14004.

[13] P. Deligne, Un théorèm de finitude pour la monodromie, Discrete Groups in Geometry and Analysis, Progr. Math., 67, Birhäuser, 1987, 1-19, MR 88h:14013, Zbl 0656.14010.

[14] S.K. Donaldson, Infinite determinants, stable bundles and curvature, Duke Math. J. 54 (1987) 231-247, MR 88g:32046, Zbl 0627.53052.

[15] R. Hamilton, Harmonic maps of manifolds with boundary, Lecture Notes in Math. 471, Springer-Verlag, Berlin and New York, 1975, MR 58 \#2872, Zbl 0308.35003.

[16] R. Hartshorne, Algebraic geometry, Graduate Texts in Mathematics, 52, SpringerVerlag, New York-Heidelberg, 1977, MR 57 \#3116, Zbl 0367.14001. 
[17] R. Hartshorne, Stable reflexive sheaves, Math. Ann. 254 (1980) 121-176, MR 82b:14011, Zbl 0431.14004.

[18] N.J. Hitchin, The self-duality equations on a Riemann surface, Proc. London Math. Soc. 55 (1987) 59-126, MR 89a:32021, Zbl 0634.53045.

[19] L. Hörmander, An introduction to complex analysis in several variables, NorthHolland Publishing Co., Amsterdam, 1990, MR 91a:32001, Zbl 0685.32001.

[20] S. Ito, Functional Analysis (in Japanese), Iwanami Shoten, Tokyo, 1983, MR 87k:47002, Zbl 0658.47002.

[21] J. Jost, J. Li \& K. Zuo, Harmonic bundles on quasi-compact Kähler manifolds, math.AG/0108166.

[22] M. Kashiwara, The asymptotic behavior of a variation of polarized Hodge structure, Publ. Res. Inst. Math. Sci. 21 (1985) 853-875, MR 87h:32049, Zbl 0594.14012.

[23] M. Kashiwara, A study of variation of mixed Hodge structure, Publ. Res. Inst. Math. Sci. 22 (1986) 991-1024, MR 89i:32050, Zbl 0621.14007.

[24] M. Kashiwara, Poincaré lemma for a variation of polarized Hodge structure, Lecture Notes in Math., 1246, Springer, Berlin, 1987, 115-124, MR 89e:32033, Zbl 0625.14005.

[25] M. Kashiwara \& T. Kawai, The Poincaré lemma for variations of polarized Hodge structure, Publ. Res. Inst. Math. Sci. 23 (1987) 345-407, MR 89g:32035, Zbl 0629.14005.

[26] S. Kobayashi, Differential geometry of complex vector bundles, Princeton University Press, Princeton, NJ; Iwanami Shoten, Tokyo, 1987, MR 89e:53100, Zbl 0708.53002.

[27] K. Kodaira, A differential-geometric method in the theory of analytic stacks Proc. Nat. Acad. Sci. USA 39 (1953) 1268-1273, MR 16,618b, Zbl 0053.11701.

[28] M. Maruyama and K. Yokogawa, Moduli of parabolic stable sheaves, Math. Ann. 293 (1992) 77-99, MR 93d:14022, Zbl 0735.14008.

[29] T. Mochizuki, Asymptotic behaviour of harmonic bundles of log type with nilpotent residue, preprint, available from http://www.math.ias.edu/ takuro/list.html.

[30] R. Palais, Foundations of global non-linear analysis, Benjamin, 1968, MR 40 \#2130, Zbl 0164.11102.

[31] G. de Rham, Differentiable manifolds. Forms, currents, harmonic forms, Grundlehren der Math. Wiss., 266, Springer-Verlag, Berlin, 1984, MR 85m:58005, Zbl 0534.58003.

[32] W. Schmid, Variation of Hodge structure: the singularities of the period mapping, Invent. Math. 22 (1973) 211-319, MR 52 \#3157, Zbl 0278.14003. 
[33] B. Shiffman \& A. J. Sommese, Vanishing Theorems on Complex Manifolds, Progress in Math. 56, Birkhäuser, 1985, MR 86h:32048, Zbl 0578.32055.

[34] C. Simpson, Constructing variations of Hodge structure using Yang-Mills theory and application to uniformization, J. Amer. Math. Soc. 1 (1988) 867-918, MR 90e:58026, Zbl 0669.58008.

[35] C. Simpson, Harmonic bundles on noncompact curves, J. Amer. Math. Soc. 3 (1990) 713-770, MR 91h:58029, Zbl 0713.58012.

[36] C. Simpson, Mixed twistor structures, math.AG/9705006.

[37] C. Simpson, The Hodge filtration on nonabelian cohomology, Algebraic geometrySanta Cruz 1995, 217-281, Proc. Sympos. Pure Math., 62, Part 2, Amer. Math. Soc., Providence, RI, 1997, MR 99g:14028, Zbl 0914.14003.

[38] K. Uhlenbeck, Connections with $L^{p}$ bounds on curvature, Comm. Math. Phys. 83 (1982) 31-42, MR 83e:53035, Zbl 0499.58019.

[39] K. Uhlenbeck and S. T. Yau, On the existence of Hermitian Yang-Mills connections in stable bundles, Comm. Pure Appl. Math., 39-S (1986), 257-293, MR 88i:58154, Zbl 0615.58045.

[40] K. Yokogawa, Compactification of moduli of parabolic sheaves and moduli of parabolic Higgs sheaves, J. Math. Kyoto Univ. 33 (1993) 451-504, MR 94h:14013, Zbl 0798.14006.

Osaka City University

OSAKA 558-8585, JAPAN

Institute for Advanced Study

Princeton, NJ 08540 